Georg- August- Universität Göttingen

e 22 次 10

\title{
Erhaltung der Bodenfruchtbarkeit unter Anwendung angepasster Anbausysteme in Bergregionen Vietnams
}

Dissertation zur Erlangung des Doktorgrades

der Mathematisch-Naturwissenschaftlichen Fakultät der Georg-August-Universität zu Göttingen

Vorgelegt von

M.Sc. Do Thi Lan

aus Son La Provinz/Vietnam 
D 7

Referentin/Referent: Prof. Dr. G. Gerold

Korreferentin/Korreferent: Prof. Dr. K.-H. Pörtge

Tag der mündlichen Prüfung: 19.01.2004 


\section{Vorwort}

Bodenerosion ist ein weltweites Phänomen, das insbesondere in Entwicklungsländern zu einer irreversiblen Bodendegradation führen kann und die Ernährungs- und Subsistenzsituation der ländlichen Bevölkerung kontinuierlich verschlechtert. Vietnam gehört zu den tropischen Ländern, in denen durch den Bevölkerungsanstieg und einer ohnehin hohen Bevölkerungsdichte im ländlichen Raum (500 E/ $\left.\mathrm{km}^{2}\right)$ der Prozeß der Waldrodung und Inkulturnahme von steileren Hanglagen im Bergland mit traditionellen Anbausystemen in den letzten 20 Jahren stark zugenommen hat. Nach FAO werden 49\% der Landesfläche als degradiert eingestuft, überwiegend durch Entwaldung und fluvialen Bodenabtrag. Mit der Dissertation wird exemplarisch für den Distrikt Ba Be im nördlichen Bergland von Vietnam die Auswirkung der Bodenerosion auf die Bodenfruchtbarkeit und die Maiserträge der lokalen Bevölkerung (ethnische Minderheiten Tay und Nung) unter Berücksichtigung alternativer Nutzungssysteme (Agroforst-Mulchsysteme) untersucht. Unter Berücksichtigung angepasster einfacher Forschungstechniken zur Erfassung der Bodenerosion wurden durch die Kombination von Feldmessungen und PRA-Ansätzen mit Haushaltserhebungen wertvolle Ergebnisse zum Entwicklungssyndrom „Bevölkerungsanstieg-Bodenerosion-Ertragsrückgang-Verarmung“ erzielt und mit einfachen angepassten Agroforstsystemen Möglichkeiten einer Verbesserung der Subsistenzwirtschaft aufgezeigt.

Es bleibt zu hoffen, dass diese Ergebnisse der Dissertation über die zukünftige Tätigkeit an der Universität Thainguyen und über die landwirtschaftlichen Beratungsstellen bei den ethnischen Minderheiten des nordvietnamesischen Berglandes erfolgreich umgesetzt werden können.

Göttingen, im März 2004

Prof. Dr. Gerhard Gerold 


\section{Danksagung}

Die vorliegende Arbeit wurde im Oktober 2000 in der Abteilung Landschaftsökologie am Geographischen Institut der Universität Göttingen begonnen und im März 2004 abgeschlossen. Dem ÖKUMENischen STUDIENWERK sei für die finanzielle Unterstützung gedankt und besonders Frau SUSANNE WERNER für ihre freundliche Begleitung während des ganzen Aufenthaltes in Deutschland.

Herrn Prof. Dr. G. Gerold hat diese Arbeit initiiert und von Anfang an thematisch angeregt. Für die inhaltliche Betreuung der Arbeit, für die allgemeine Unterstützung möchte ich ihm besonders danken. Prof. Dr. NGuYen The DANG gilt mein herzlicher Dank für die Hilfe bei der Feldforschung in Vietnam und Herrn Dr. Michael WAIBEL sowie Herrn Dr. STEPHAN GLATZEL gebühren vor allem mein Dank für die freundliche Hilfsbereitschaft bei der schwierigen deutschen Stilistik und Rechtschreibung.

Für die Laboranalyse danke ich Herrn Dr .J. GROTHEER, Frau P. VoIGT und Frau A. SöDJE für ihre freundliche Hilfsbereitschaft. Nina Rohwer, AnNa Purschwitz und Peter Just sei ebenfalls für ihre Hilfe bei Arbeiten im Labor, Kartenerstellung und Textkorrektur gedankt. Weiterhin möchte ich allen Mitarbeiterinnen und Mitarbeitern des Institutes für die Hilfsbereitschaft und Kameradschaft meinen herzlichen Dank aussprechen.

Mit großer Dankbarkeit denke ich gern an die herzliche Atmosphäre und die entspannte Zusammenarbeit von Kollegen und Kolleginnen in der Fakultät Landmanagement, Thai Nguyen Universität zurück.

Zum Gelingen dieser Arbeit haben Mitarbeiterinnen und Mitarbeiter des Department für Statistik sowie des land- und forstwirtschaftlichen Department und der Klimastation im Distrikt Babe, ebenso die Familien in den untersuchten Dörfern beigetragen, die mir gegenüber sehr offen und freundlich waren. Ihnen danke ich sehr.

Schließlich möchte ich meinem Mann und meiner Tochter danken für die Zeit, die sie mir zum Anfertigen der Arbeit ließen, als auch für ihre Geduld und Bemühungen, mich daran zu erinnern, dass es neben der wissenschaftlichen Arbeit auch noch andere Dinge im Leben gibt. Sie gaben mir den Mut, diese Arbeit anzufertigen. 


\section{Inhaltsverzeichnis}

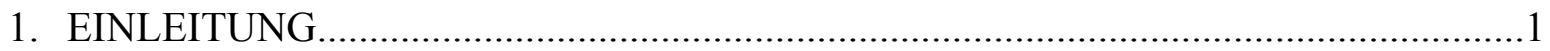

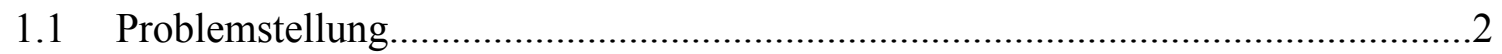

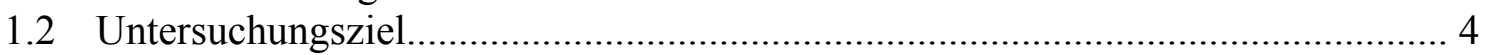

1.2.1 Gemeinsame Ziele für Vietnam..........................................................4

1.2.2 Die wichtigsten Ziele dieser Untersuchung ...........................................

2. NUTZUNGSSYSTEME UND BODENEROSION 6

2.1 Shifting cultivation system - traditionelle Nutzungsysteme in Nordvietnam..........6

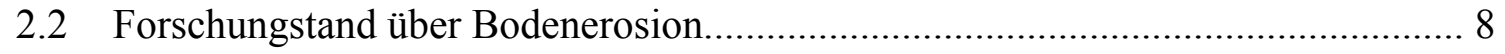

2.3 Bedeutung von Erosion und Erosionsschutzmaßnahmen................................... 10

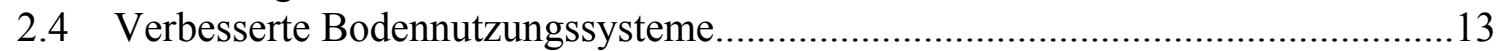

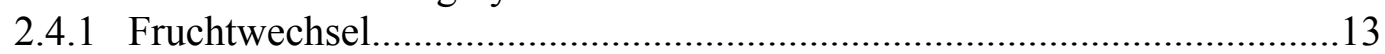

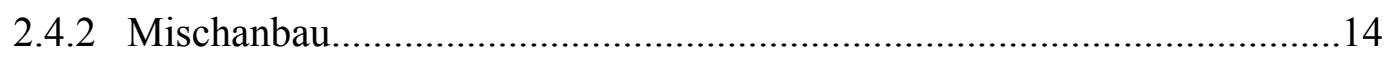

2.4.3 Agroforstliche Systeme ( Agrofostwirtschaft)......................................14

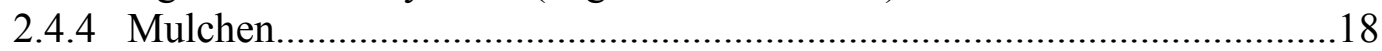

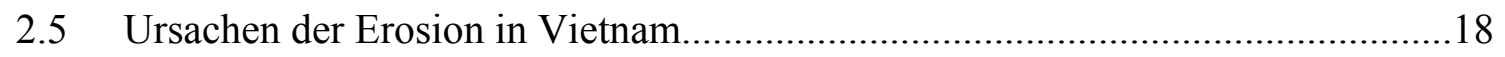

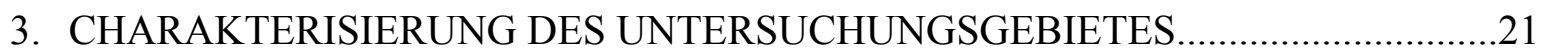

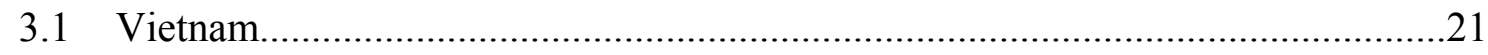

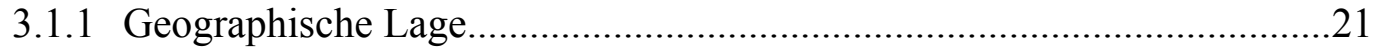

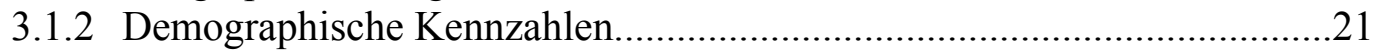

3.1.3 Situation Boden und Landnutzung.....................................................22

3.1.4 Reformen im landwirtschaftlichen Sektor..............................................22

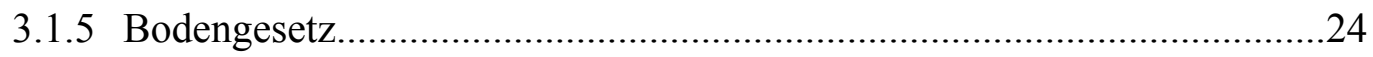

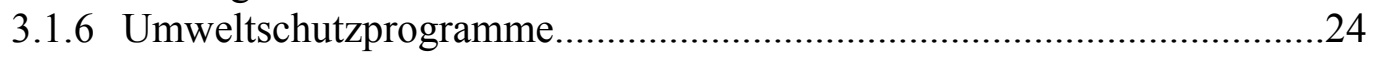

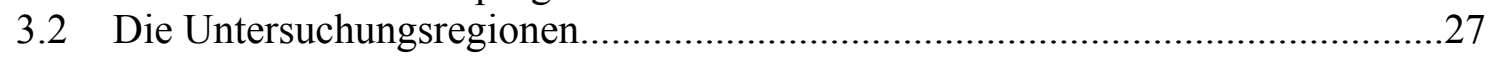

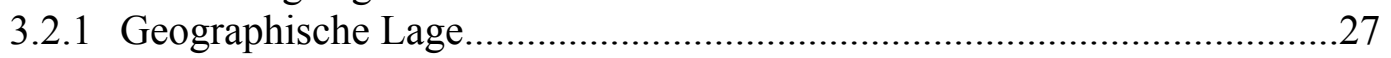

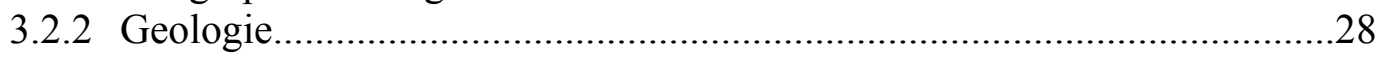

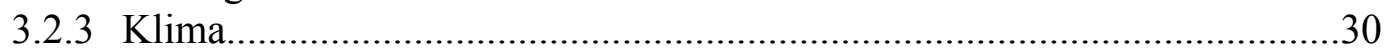

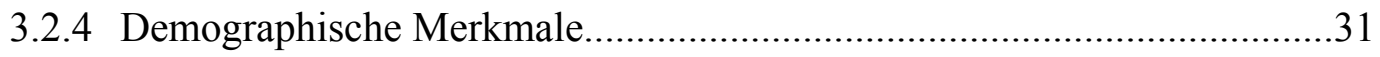

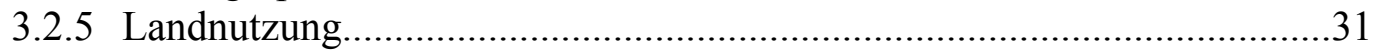

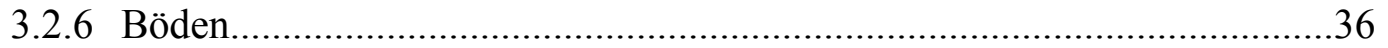

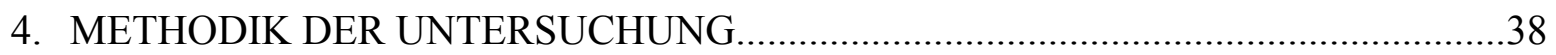

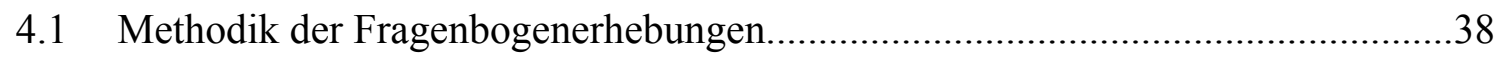

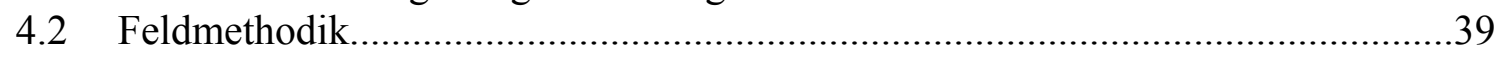

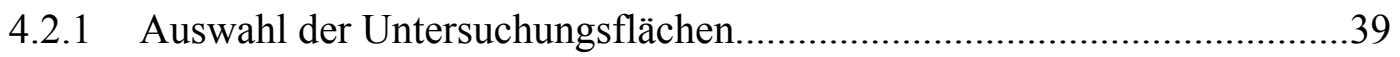

4.2.2 Chronosequenz: Beprobung der Untersuchungsflächen..........................40

4.2.3 Versuchstandort und Methodik................................................................4 41

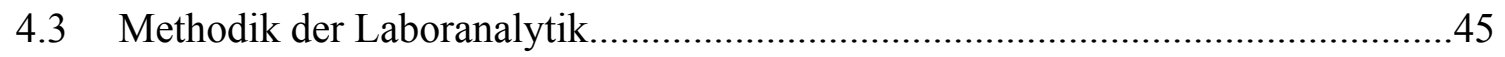

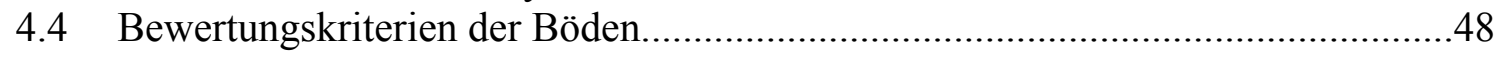

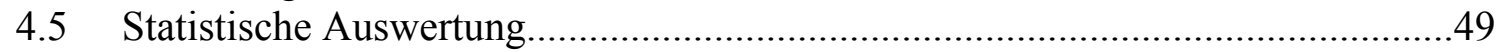

5. SOZIOÖKONOMISCHE ANALYSE DER LANDWIRTSCHAFTLICHEN

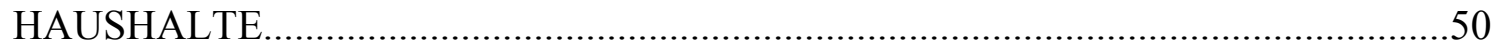

5.1 Klassifizierung und Auswahl von Haushalten für die Fragebogen-Erhebung......50

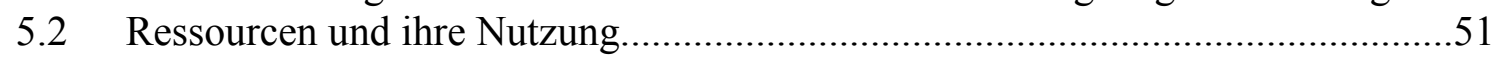

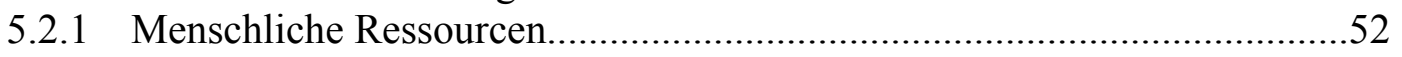

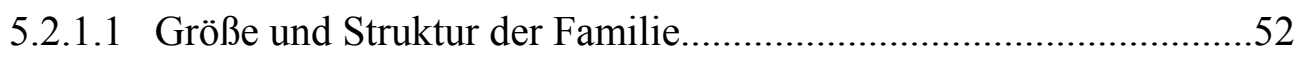

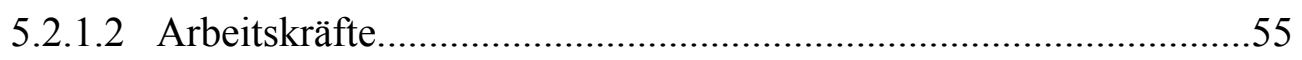




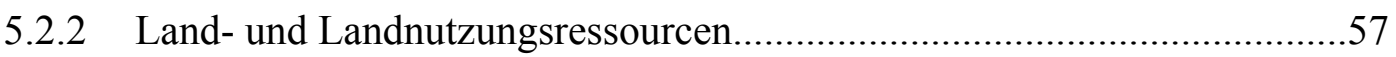

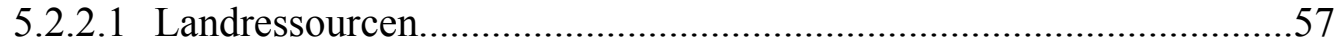

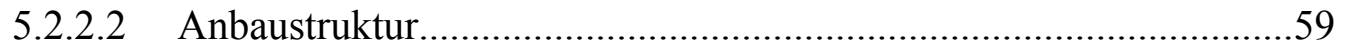

5.2.2.3 Jahreskalender der Familien in Untersuchungsgebiet.......................62

5.2.2.4 Geschlechtsspezifische Arbeitsteilungen bei den Tay.......................64

5.2.2.5 Bodenqualität und nachhaltige Nutzung........................................66

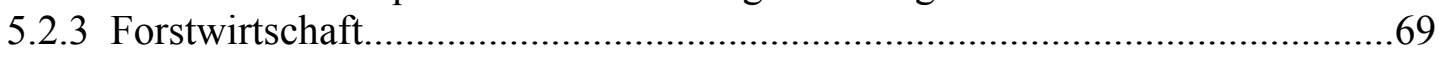

5.2.3.1 Durchschnittliche Waldfläche jeder Familie................................... 69

5.2.3.2 Waldnutzung durch die Bauern.....................................................69

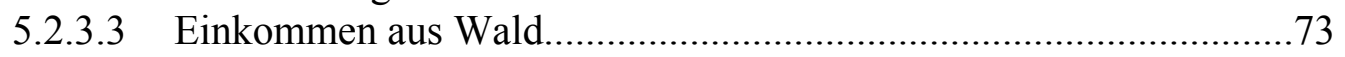

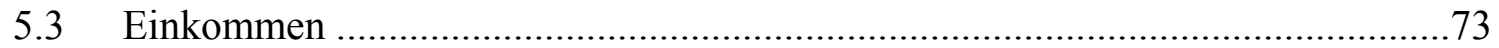

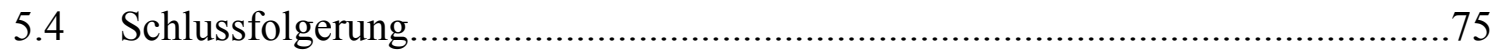

6. ERGEBNIS DER FELDUNTERSUCHUNG UND IHRE BEWERTUNG.................76

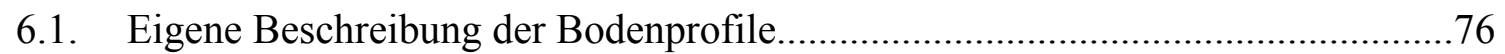

6.2 Bodeneigenschaften und Bodenqualität im Untersuchungsgebiet........................85

6.3 Bodenfruchtbarkeitsparameter in Abhängigkeit von der Nutzungsdauer...............90

6.3.1 pH und Basensättigung......................................................................90

6.3.2 Organische Substanz und Gesamtstickstoff..............................................93

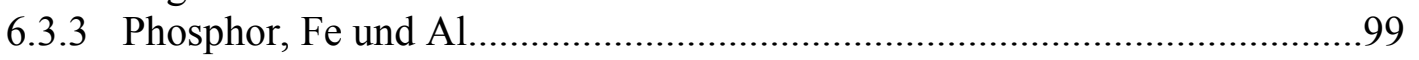

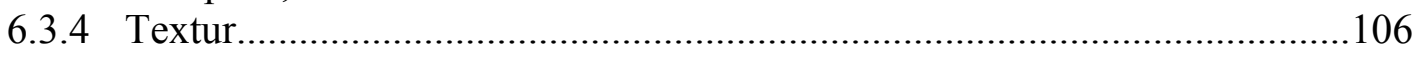

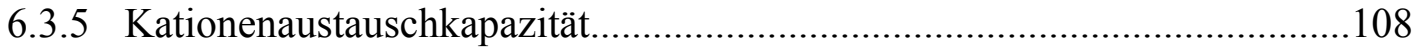

6.3.6 Andere Parameter............................................................................. 114

6.4 Schlussfolgerung: Einflüsse des Brandrodungsfeldbau auf die Bodenfruchtbarkeit

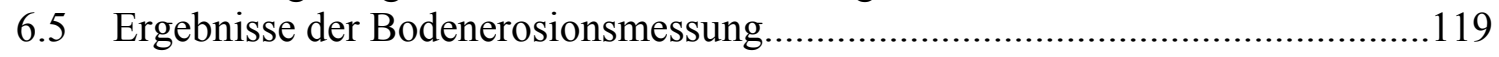

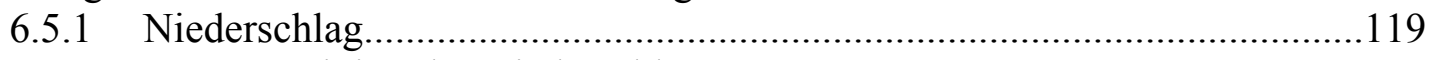

6.5.1.1 Erosivität des Niederschlages.....................................................119

6.5.1.2 Einfluss von Regenintensität und Niederschlagsmenge auf die Bodenerosion ...............................................................................122

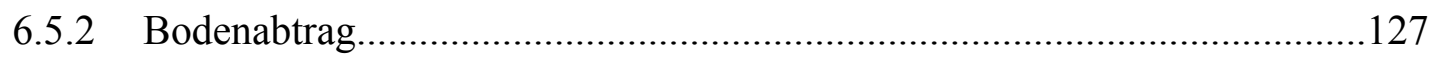

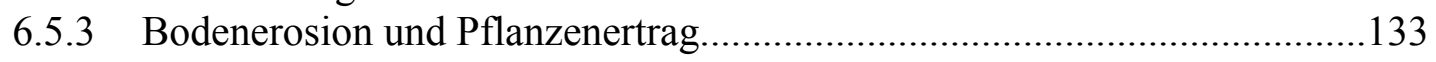

6.5.4 Art und Grad der Bodenbedeckung......................................................136

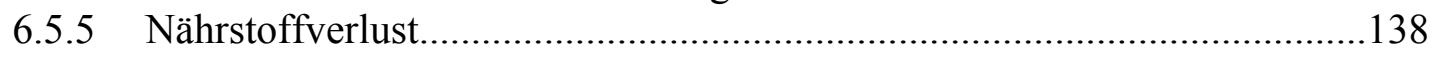

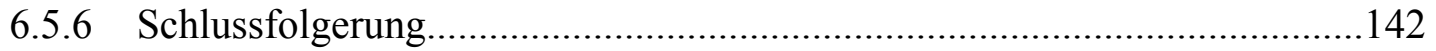

6.6 Wirkung der Schutzmaßnahme gegen Erosion und Erhaltung der

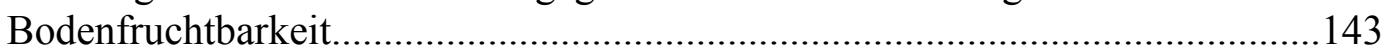

7. Modellvorschlag zur Gestaltung nachhaltiger Landnutzung......................................150

8. Aspekte der praktischen Umsetzung von Erosionsschutzmaßnahmen........................157

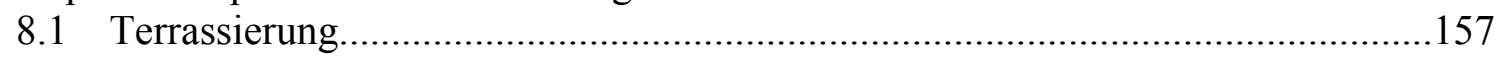

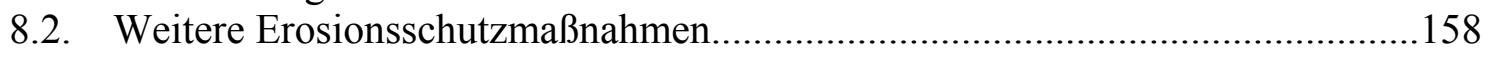

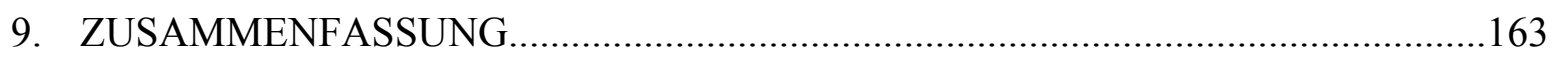

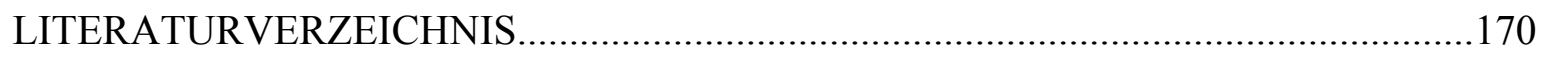

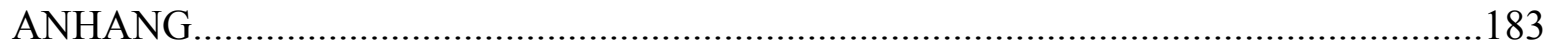




\section{Verzeichnis der Tabellen}

Tab 1: Bodendegradation in einigen asiatischen Ländern (in 1000 ha) 16

Tab.2: Länder in Asien mit hohem Anteil schwer bis extrem erodierter Flächen nach 22 Region

Tab.3: Ertrag der geschnittenen Pflanzen im Jahr (t/ha)

Tab.4: Die Veränderungen der Waldfläche in Vietnam von 1943 bis 1993

Tab.5: Schema der Bodenbeprobung 41

Tab.6: Standortangaben der vier Erosionsmessparzellen im Einzugsgebiet Dialinh, $\quad 42$ Babe Distrikt.

Tab.7: Richtwerte für die Beurteilung der KAKeff nach LANDON 1991

Tab.8: Richtwerte für die organische Substanz und den Gesamtgehalt an Stickstoff $\quad 48$ für die verschiedenen Bodenarten nach PAGEL 1982

Tab.9: Richtwerte für pflanzenverfügbare Nähstoffe und den Gesamtgehalt an $\quad 48$ Schwefel nach LANDON 1984

Tab.10: Richtwerte für die Beurteilung der Sättigungsgrade an der KAK in \% nach 49 PAGEL 1982

Tab.11: Richtwerte für die Phosphor pflanzenverfügbar nach HoFFMANN 1991

Tab.12: Wirtschaftliche Lage der Bevölkerung von Dia Linh im Jahre 2001

Tab.13: Durchschnittliche Anzahl der Personen pro Familie in Dia Linh 2001

Tab.14: Durchschnittliche Anzahl der Arbeitkräfte, differenziert nach Lebensstandard, Dia Linh 2001

Tab.15: Aktuelle Landwirtschaftsflächen von Haushalten in Dia Linh-Ba Be, 2001

Tab.16: Tatsächliche Landnutzung im Jahr 2001 im Untersuchungsgebiet

Tab.17: Obst Anbau pro Betrieb in Dia Linh-Ba Be, 2001

Tab.18: Die Bodenqualität von einzelnen Haushalten, Dia Linh-Ba Be, 2001

Tab.19: Bodenerosion nach Arte der Kulturpflanzen (\% Fläche)

Tab.20: Meinung der Bauern zu Fragen des Bodenerosionsschutzes

Tab.21: Durchschnittliche Waldfläche pro Haushalt, Dia Linh 2001

Tab.22: Vorangige Waldnutzung durch Haushalte, Dia Linh 2001

Tab.23: Durchschnittliche Einkommen aus Wald, Dia Linh 2001

Tab.24: Tatsächliche Einkommen von Familien in Dia Linh 2001

Tab.25: Textur in Abhängigkeit von der Dauer der traditionale Wanderfeldbau

Tab.26: Beziehung zwischen Niederschlag und Bodenerosion - Beobachtung einiger charakteristischer Niederschlagsereignisse

Tab.27: Beziehung zwischen Regenintensität und Oberflächenabfluss

Tab.28: Vergleich von Bodenabtrag zu Beginn und Ende der Regenzeit

Tab.29: Kenndaten zum Maisertrag und tatsächlicher Maisertrag

Tab.30: Trockenmasse von Bodenbedeckungspflanzen der Testparzelle im Dialinh, Babe 2001

Tab.31: Nährstoffverlust durch Bodenerosion

Tab.32: Kostenaufwand an NPK- Düngung zum Ausgleich von Nährstoffverlusten durch Bodenerosion

Tab.33: Richtwerte für Nährstoff - Bedarf von Mais

Tab.34: Wirkung von Gründüngungspflanzen gegen Bodenerosion

Tab.35: Nährstoff von Gründüngungspflanzen ( $\mathrm{kg} / \mathrm{ha})$

Tab.36: Vorschlag zum Management von Wald in Tay - Dörfern

Tab.37: Vorschlag zum Management von Agroforst in Tay- Dörfern

Tab.38: Wünschenswerte Eigenschaften von Gründüngungspflanzen 


\section{Verzeichnis der Abbildungen}

Abb.1: Arbeitsgebiet

Abb.2: Durchschnittliche Klimadaten des Distrikts Ba Be in den Jahren 1996-2000 30

Abb.3: Durchschnittliche Niederschlag und Evaporation des Distrikts Ba Be in den 30 Jahren 1996-2000

Abb.4: Tal-Querschnitt eines Landwirtschaftsökologiesystems vom Dorf Dia Linh, $\mathrm{Ba} \mathrm{Be}$

Abb.5: Aufforstungen in der Provinz Bac Can 1995-1999

Abb.6: Feldbau-, Sekundärbusch- und Brachefläche im Ba Be Distrikt 2001

Abb.7: Graphische Darstellung der Flächennutzung des Distriktes Ba Be

Abb.8: Messparzellen

Abb.9: Schema Kleinbäuerliches Agro-Forstsystem mit Erosionsschutzmaßnahmen

Abb.10: Bildungsniveau der Familien im Untersuchungsgebiet

Abb.11: Der Teufelkreis der Armut

Abb.12: Situation der Land besitzen, Dia Linh - Ba Be, 2001

Abb.13: Natürliche Brache im Untersuchungsgebiet in den letzten 15-30 Jahren 60

Abb.14: Natürliche Brache im Untersuchungsgebiet heute

Abb.15: Angebaute Kulturarten in den verschiedenen Betriebsgruppen in Dia Linh Ba Be, 2001

Abb.16: Jahresarbeitskalender der Familie in Dialinh, Babe District

Abb.17: Verteilung der Leitbodenprofile bei neue Landnutzungssystem (Agroforstsystem mit Mulch)

Abb.18: Verteilung der Leitbodenprofile bei traditionale Landnutzungssystem (Wanderfeldbaum mit Brandrodung)

Abb.19: Korrelation zwischen organischer Substanz und Gesamtstickstoff

Abb.20: Korrelation zwischen organischer Substanz und KAKeff 86

Abb.21: Korrelation zwischen $\mathrm{pH}(\mathrm{KCl})$ und KAKeff

Abb.22: Korrelation zwischen Phosphor und org. Substanz

Abb.23: Korrelation zwischen gesamte Phosphor und Fe

Abb.24: Korrelation zwischen Phosphorsverfügbarkeit und Al

Abb.25: pH-Wert und Basensättigung in Abhängigkeit von Anbaudauer und Nutzungstyp

Abb.26: Die Veränderung der pH-Wert im Oberboden vor dem Anbau und nach der Ernte

Abb.27: Die organische Substanz in Abhängigkeit von Anbaudauer und Nutzungstyp

Abb.28: Gesamtstickstoff in Abhängigkeit von Anbaudauer und Nutzungstyp Oberboden

Abb.30: C/N-Ratio in Ober- und Unterboden in Abhängigkeit von Anbaudauer und Nutzungstyp

Abb.31: Gesamt -P-Gehalt in Abhängigkeit von Anbaudauer und Nutzungstyp

Abb.32: Die Veränderung von P-pflanzenverfügbar in Abhängigkeit von Anbaudauer und Nutzungstyp

Abb.33: Die Veränderung von P-pflanzenverfügbar vor dem Anbau und nach der Ernte im Oberboden

Abb.34: Der Phosphorkreislauf während der Wachstumsperiode in shifting cultivation

Abb.35: Korrelation zwischen $\mathrm{pH}$ und P-pflanzenverfügbar 
Abb.36: Spezifische Adsorption von Phosphat an Eisen- oder Aluminiumoxide $\quad 104$

Abb.37: Veränderung von Al, Fe und P in einem Zyklus des traditionellen 105 Wanderfeldbaues, Alang Alang Brache und Agroforst mit Mulch

Abb.38: Veränderung von $\mathrm{Fe}$ und $\mathrm{Al}$ in einem Zyklus des traditionellen

Wanderfeldbaues, Alang-Alang-Brache und Agroforst mit Mulch

Abb.39: Fe, Al vor dem Anbau und nach der Ernte im Oberboden 106

Abb.40: KAKeff in Abhängigkeit von Nutzungsdauer und Nutzungstyp 108

Abb.41: Austauschbares Ca in Ober- und Unterboden 109

Abb.42: Austauschbares Mg in Ober - und Unterboden $\quad 110$

Abb.43: Korrelation zwischen austauschbarem Ca und der Austauschkapazität $\quad 110$

Abb.44: KAKeff vor dem Anbau und nach der Ernte im Oberboden 112

Abb.45: Gesamtgehalte in Abhängigkeit von der Dauer des Wanderfeldbau $\quad 114$

Abb.46: Monatlicher Niederschlag von April - Oktober 2001 in Dia linh 120

Abb.47: Die Verteilung und Höhe der Niederschlagsereignisse während der 121 Regenzeit

Abb.48: Bodenabtrag verschiedener Landnutzungssysteme in $2001 \quad 128$

Abb.49: Fortschreiten von Bodendegradation und -erosion 132

Abb.50: Wachstum von Mais im 1. und 4. Jahr nach Brandrodung (Testparzelle 134 Dialinh, Babe)

Abb.51: Wirkungen von Mulch auf die Bodenfruchtbarkeit 145

Abb.52: Modellvorschlag zur Gestaltung nachhaltiger Landnutzung 151

Abb.53: Verteilung von Land-und Forstwirtschaft in Abhägigkeit von der 153

Hangneigung

Abb.54: Konturstreifenanbau $\quad 154$

Abb.55: A-Rahmen: Einfache Geräte zur Vermessung von Höhenlinien 158

Abb.56: Synthese: Ein agroforstliches Rotationsystem 158

Abb.57: Hypothesenstern $\quad 162$

\section{Verzeichnis der Figuren}

Fig.1: Leitprofil 1: Agroforstsystem mit Mulch 77

Fig.2: Leitprofil 2: Nur Alang Alang Bewuchs $\quad 78$

Fig.3: Leitprofil 3: 1. Jahr nach Brandrodung $\quad 79$

Fig.4: Leitprofil 4: 4. Jahr nach Brandrodung $\quad 80$

Fig.5: Leitprofil 5: 4. Brachejahr (Sekundärbusch) 81

Fig.6: Leitprofil 6: Sekundärwald $\quad 82$

\section{Verzeichnis der Karten}

Karte 1: Karte 1: Wichtigste unmittelbare Erosionsursache nach mapping unit 20

Karte 2: Die Veränderungen der Waldfläche in Vietnam nach 54 Jahre 26

Karte 3: Geologisch - geomorphologische Verhältnisse im Dia linh, Babe 29

Distrikt

Karte 4: $\quad$ Anteil erodierter Fläche pro mapping unit 183

Karte 5: $\quad$ Haupterosionstyp pro mapping unit 183 


\section{Verzeichnis der Abkürzungen}

$\mu \mathrm{m}$ Mikrometer

$\mu \mathrm{S}$ Mikrosiemens

AAS Atomabsorptions-Spektrometer

Agro. M Agroforstsystem mit Mulch

AG Arbeitsgemeinschaft

AgSt Aggregatstabilität

Ah humoser Mineralbodenhorizont ( Humusgehalt 0,6-15 Masse \%) in A-Horizont

Ap A-Horizont durch regelmäßige Bodenbearbeitung geprägt, Pflughorizont

$\mathrm{BaCl}_{2}$ Bariumchlorid

BMZ Bundesministerium für Wirtschaftliche Zusammenarbeit und

BS Basensättigung

Bws- Horizont: Tonfraktion besteht vorwiegend aus LAC( Kaolinit), Schluff- und Sandfraktion enthält verwitterungsresistente Minerale (Fe-, Al-, Mn-Oxide) und mit Sesquioxiden durch Umlagerung angereicherter B-Horizont

bzw. beziehungsweise

$\mathrm{CaCl}_{2}$ Calciumchlorid

$\mathrm{CaCO}_{3}$ Calciumcarbonat

cmolc/kg Einheit der KAK: Centimol Ladung pro Kilogramm

Corg. Organischer Kohlenstoff

d.h. das heißt

DIN Deutsche Industrienorm

DWI Durchwurzelungsintensität

Einz. Einzelkorngefüge

FAO Food and Agriculture Organization of the United Nations

FK Feldkapazität

FRg Geric Ferralsol

FRu Humic Ferralsol

FRr Rhodic Ferralsol

Gew.-\% Gewichtsprozent

GmbH Gesellschaft mit beschränkter Haftung

GLASOD Global Assessment of Soil Degradation

GTZ Deutsche Gesellschaft für Technische Zusammenarbeit, Eschborn

gU Grobschluff

$\mathrm{H}_{2} \mathrm{O}_{2}$ Wasserstoffperoxid

$\mathrm{HCl}$ Salzsäure

$\mathrm{HClO}_{4}$ Perchlorsäure

HF Flusssäure

$\mathrm{HNO}_{3}$ Salpetersäure

KAKeff. effektive Kationenaustauschkapazität

KAK pot. potentielle Kationenaustauschkapazität

Kap. Kapitel

KCl Kaliumchlorid

koh. Kohärentgefüge

Kr Krümelgefüge

L- Horizont: Frisch gefallene, nur wenig zersetzte Streu, Struktur noch weitgehend intakt.

Ld Lagerungsdichte

LK LuftkapazitätLK Luftkapazität

Lts sandig-toniger Lehm

Max. Maximum 
$\mathrm{MgCl} 2$ Magnesiumchlorid

Min. Minimum

$\mathrm{mS}$ Mittelsand

mU Mittelschluff

$\mathrm{N}$ Niederschlag

$\mathrm{Na}_{4} \mathrm{P}_{2} \mathrm{O}_{7}$ Natriumpyrophosphat

$\mathrm{NaHCO}_{3}$ Natriumhydrogencarbonat

nFK nutzbare Feldkapazität

nFKWe nutzbare Feldkapazität des effektiven Wurzelraumes

$\mathrm{NH} 4$ Ammonium

$\mathrm{Nt}$ Gesamtstickstoff

o.a. oben angegeben

pH negativer dekadischer Logarithmus der $\mathrm{H}_{3} \mathrm{O}+-$ Konzentrationen

ppm parts per million

PRA Participatory Rapid Rural Appraisal

$\mathrm{r}^{2}$ Korrelationskoeffizient (Bestimmtheitsmaß)

RRA Rapid Rural Appraisal

S. Seite

Lt3: mittel toniger Lehm

$\mathrm{Tu} 2$ schwach schluffiger Ton

Tu3 mittel schluffiger Ton

Us sandige Schluff

u.a. unter anderem

UNESCO United Nations Educational, Scientific and Cultural Organization

üNN über Normalnull

URL Uniform Resource Locater

US-\$ Amerikanische Dollar

USLE Universal Soil Loss Equation

VDLUFA Verband Deutscher Landwirtschaftlicher Untersuchungs-und Forschungsanstalten vgl. vergleiche

Vol.-\% Volumenprozent

Y Yellow

z.B. zum Beispiel

zit. zitiert 


\section{EINLEITUNG}

Weltweit gibt es mehr als 800 Mio. Menschen, die unterernährt sind und unter Hunger leiden, obwohl in den letzten Jahrzehnten ein großer technischer Fortschritt in der Agrarproduktion erzielt wurde. Diese paradoxe Situation hat viele Ursachen. (BOHNET, 2001). Die beunruhigendste Ursache besteht in der Tatsache, dass die Produktionskapazität von landwirtschaftlichen Nutzflächen durch unangepasste Bodennutzungssysteme und fehlerhafte Bewirtschaftung herabgesetzt wird oder gar verloren geht.

Weltweit weisen rund 1.965 Mio. ha Bodenfläche Degradationserscheinungen auf, deren schwerwiegendste Ursache die Abspülung oder Wassererosion ist. Die auslösenden Kräfte sind der Aufprall der Regentropfen und die Schleppkraft des abfließenden Wassers. Diese Form der Bodenerosion führt $\mathrm{zu}$ einer Verringerung der Bodenfruchtbarkeit, im Extremfall zu einer Zerstörung. Nach Angaben der Vereinten Nationen büßen jedes Jahr weltweit 20 Mio. ha Ackerland ihre Bodenfruchtbarkeit durch Erosion ein. Die gesamte Fläche, die durch menschlich verursachte Bodenerosion beeinträchtigt wird, beläuft sich auf geschätzte 1.094 Mio. ha (DOPPLER \& BAUER, 2001) Die Bodenerosion ist in den letzten Jahrzehnten durch die Änderung und Intensivierung der landwirtschaftlichen Nutzung beschleunigt worden. Gründe für die Zunahme sind u.a. die Vergrößerung der Schläge unter Beseitigung der vor Erosion schützenden Hangstufen und Hecken, die Zunahme der Früchte mit langer Bedeckung und eine intensive Bodenbearbeitung, die den Humusabbau beschleunigt und die Verschlämmung des Bodens fördert (AID, 1993).

Aufgrund der global fortschreitenden Vegetations- und Bodendegradation sowie der verstärkten internationalen Bemühungen zur nachhaltigen Entwicklung werden über Entwicklungs- und Forschungsprojekte nachhaltige Landnutzungssysteme für die Tropen propagiert und umgesetzt. Um das Ziel einer nachhaltigen Landnutzung zu erreichen, müssen irreversible Änderungen im Wasser- und Nährstoffumsatz vermieden werden. Die Kenntnisse über geoökologische Grundlagen wie Bodenqualität sowie Wasser- und Nähstoffflüsse in den Tropen sind eine Voraussetzung für die regionale Anpassung experimentell abgeleiteter Anbauverbesserungen (GEROLD, 2002).

Die ökologischen und ökonomischen Folgen der Bodenerosion sind vielfältig und umfassen sowohl Schäden auf der erodierten Ackerfläche (Onsite- Schäden) als auch benachbarter Ökosysteme, in denen sich erosionsbedingte Stoffeinträge negativ 
auswirken (Offsite- Schäden). Onsite- Schäden umfassen neben Nährstoff- und Ertragsverlusten auch einen Wertverlust des Bodens. Unter den Offsite- Schäden ist die Verschmutzung von Gewässern sehr bedeutend. Sie wird zum großen Teil auf diffuse Austräge aus der Landwirtschaft zurückgeführt, wobei ein großer Anteil erosionsbedingt ist (DOPPDER et al, 1991).

Für die Tropen, insbesondere die Inneren Tropen, stellt sich daher die Frage, inwieweit diese negativen Veränderungen durch die Entwicklung angepasster Bodennutzungssysteme unter Berücksichtigung der regionalen Bodenqualitätsunterschiede abgeschwächt oder vermieden werden können (z.B. AgroForstsysteme mit C-Speicherung und Vermeidung von Bodenerosion). Die Kenntnisse der veränderten Wasser- und Nährstoffumsätze und geoökologischer Grundlagen im Vergleich von Wald und Nutzungssystem sind dafür eine wichtige Voraussetzung (GEROLD, 2002). Die Degradation landwirtschaftlicher Nutzflächen durch den Abtrag fruchtbaren Oberbodens (Bodenerosion) ist ein weltweit beobachtbares Phänomen. Sie wird insbesondere in Entwicklungsländern zum Problem, da ein Verlust der Ressource Boden dort die ohnehin unsichere Ernährungssituation weiter Bevölkerungsteile zusätzlich gefährdet (PIMENTEL et al., 1995, S.117; PrinstruP-ANDERSEN et al., 1997, S.5).

\subsection{Problemstellung}

Bodendegradierung durch Erosion ist in vielen Entwicklungsländern ein schwerwiegendes Problem. Viele Böden verlieren ihre natürliche Bodenfruchtbarkeit durch die Anwendung landwirtschaftlicher Bodennutzungssysteme, die nicht an die natürlichen Bedingungen angepasst sind. Langfristig gesehen kann dadurch die Ernährungssicherung der Bevölkerung gefährdet sein.

Vietnam gehört zu den Ländern mit der geringsten landwirtschaftlichen Nutzfläche pro Einwohner, im Landesdurchschnitt sind es 0,1 ha (Statistisches Jahrbuch, 1999). Der Druck auf die Ressourcen Land und Wald ist entsprechend hoch. Die Bergregionen Vietnams sind Siedlungsgebiete für eine Vielzahl ethnischer Minoritäten. Im Untersuchungsgebiet, dem Distrikt $\mathrm{Ba} \mathrm{Be}$, handelt es sich überwiegend um Tay und Nung, die in den Tallagen siedeln, sowie um Hmong und Dao, deren Dörfer meist in den Bergen über 700 m ü. N.N. liegen. Die Kinh, ethnische Vietnamesen, wurden in den 60er Jahren vom Deltagebiet des Roten Flusses hierher umgesiedelt. Ethnien, die vornehmlich 
die Bergregionen bewohnten und deren Anbautechnik seit Jahrhunderten der Wanderfeldbau war, wurden gezwungen, sich dauerhaft an einem Ort niederzulassen.

Die Fläche Vietnams beträgt 33,1 Mio. ha, davon weisen über 20 Mio. ha, also ca. 2/3 der Landesfläche, eine Hangneigung über $15^{\circ}$ auf. Die durchschnittliche Regenmenge beträgt ca. $1.500 \mathrm{~mm} / \mathrm{Jahr}$. 75 bis $80 \%$ der Niederschläge konzentrieren sich auf die Regenzeit von ca. 3 Monaten. Stellenweise regnet es bis zu 245 mm/Tag. Das Zusammenspiel dieser Gegebenheiten verursacht schwerwiegende Bodenerosion in den Gebirgsgegenden des Landes. Der ökologische Zustand der Bergregionen ist deshalb besorgniserregend. Die heutige bewaldete Gesamtfläche wird mit mehr als 11 Mio. ha angegeben und insgesamt können 12,9 Mio. ha des Landes derzeit nicht landwirtschaftlich genutzt werden (THAI. \& NGUYEN., 1998).

Um mehr Ertragssicherheit zu erlangen und um die Nahrungsmittelproduktion dem jährlichen Bevölkerungswachstum von 1,9\% (Statistisches Jahrbuch, 2000) anzupassen, muss einerseits die agrarisch genutzte Fläche weiter ausgedehnt, und andererseits die Flächenerträge durch intensivere Bewirtschaftung erhöht werden. Bevölkerungswachstum, Migrationbewegungen aus den Flachlandregionen in die Bergregionen, ungeeignete Bewässerungsprojekte und Umsiedlungsprogramme der Regierung haben in den vergangenen Jahren dazu geführt, dass die Bevölkerungsdichte stark zugenommen und die durchschnittliche Flächenverfügbarkeit pro Person abgenommen hat. Die Folgen sind eine Zunahme der Abholzungsrate und eine Intensivierung der Flächennutzung. Zum einen wurden Bracheperioden reduziert, zum anderen auch marginale Flächen genutzt, die für permanenten Regenfeldbau wenig geeignet sind. Es wurde beobachtet, dass reduzierte Bracheperioden $\mathrm{zu}$ einer verminderten Regeneration der Böden in Bergregionen führte. Diese Regionen wurden schon zuvor durch den weit verbreiteten Wanderfeldbau, aggressive Abholzungspraktiken und Folgen der vergangenen Kriege stark belastet.

Immigranten aus dem Tiefland Vietnams verfügen oft nicht über Kenntnisse, die für eine standortgerechte Bewirtschaftung der landwirtschaftlichen Nutzfläche in Hanglagen vonnöten wären. Je stärker die Migration aus dem Tiefland in die Bergregionen ist, desto schneller verringert sich das Bodennutzungspotential in den Gebirgen.

Das Problem verschärft sich, weil in vielen Regionen Vietnams große Mengen an Niederschlag (1500-3000 mm/a) in sehr kurzer Zeit (Juni-September) fallen. Einzelne Starkregenereignisse erreichen Niederschlagsmengen von $300 \mathrm{~mm}$ in wenigen Stunden. Stärkste Bodenerosion auf unbedeckten Flächen ist die Folge (THAI \& NGUYEN, 1999). 
Ein großes Problem besteht darin, dass die Bevölkerung nur wenig Information über die Ursachen, die Gefahren und Folgen der Bodenerosion erhält. Der Babe Distrikt in der Bac Can Provinz in Nordvietnam ist eine solche Region, in der Prozesse der Bodendegradation, wie nährstoffverarmte Böden, Veränderung der bodenphysikalischen Eigenschaften, Ausbreitung konkurrenzkräftiger Unkräuter, Zerstörung von Wäldern, Verringerung von Brache und Ihre Auswirkungen besonders stark und offensichtlich sind. In diesen Bergregionen wird die Landwirtschaft vor allem durch sehr geringe Bodenfruchtbarkeit und hohe Erosionsgefahr auf steilen Hängen beeinträchtigt. Viele bäuerliche Familien in der Region verfügen nur über ein geringes Einkommen und leiden unter Armut. Um die genannten Problem zu überwinden, ist die Entwicklung nachhaltigerer Flächennutzungssysteme, in deren Zentrum der Erhalt der natürlichen Bodenfruchtbarkeit und die Überwindung der Armut stehen, ein dringende Notwendigkeit.

\subsection{Untersuchungsziel}

\subsubsection{Gemeinsame Ziele für Vietnam/Bedeutung für Vietnam}

Untersuchungen zur Optimierung von Bodennutzungssystemen unter Berücksichtigung ökologischer und sozioökonomischer Faktoren sind notwendig. Zur Erhaltung der Bodenfruchtbarkeit ist daher in Vietnam folgendes erforderlich:

- Bekämpfung der Erosion, Erhöhung des Flächenertrages.

- Es wird angestrebt, durch eine Terrassierung der Hänge und der Pflanzung von Hecken eine nachhaltige und dauerhafte Bodennutzung zu erzielen.

- Auswahl effizienter und angepasster Anbausysteme zur dauerhaften Entwicklung in Bergregionen.

- Verminderung der Armut und des Hungers und Verbesserung der Kenntnisse für den Anbau mit Bodenerosionsschutz (landwirtschaftliche Beratung, Schulung).

- Einkommenserhöhung der Kleinbauern durch gesicherte landwirtschaftliche Produktion und somit Selbstversorgung, Risikominderung und Unabhängigkeit.

\subsubsection{Die wichtigsten Ziele dieser Untersuchung sind:}

1- Die sozioökonomischen Eigenschaften mit den landwirtschaftlichen Einkommen, Bedingungen und der Perzeption der Bodenerosion der Haushalte im Untersuchungsgebiet zu beschreiben.

2- die Erfassung der aktuellen Landnutzung im Untersuchungsgebiet 
3- die Auswirkungen der Bodenerosion auf chemische und physikalische Bodeneigenschaften und deren Einfluss auf die Ertragsleistung der Böden zu untersuchen: Dies beinhaltet die Ermittlung von Bodenabtragsraten, den damit verbundenen Nährstoffverlusten und deren Auswirkungen auf den Pflanzenertrag in Abhängigkeit von Oberflächenabfluss, Niederschlag und Dauer der Bodennutzung.

4- Erhaltung bzw. Verbesserung der Bodenfruchtbarkeit und somit Erhöhung der Erträge und Gewährleistung einer langfristigen Lebensgrundlage durch Anwendung bodenerosionsminimierender Anbautechniken.

5- Entwicklung effizienter und angepasster Anbausysteme zur dauerhaften Entwicklung in Bergregionen.

Dies setzt voraus, dass aus den Ergebnissen der Bodenerosionsgefährdung in Kooperation mit den Bauern tragfähige, an die natürlichen und ökonomischen Bedingungen angepasste Anbausysteme entwickelt werden sollen.

Konkret stellte sich für diese Studie einerseits die Frage, inwiefern agroforstliche Maßnahmen einen Beitrag zur Erhöhung oder zumindest Stabilisierung der Bodenfruchtbarkeit leisten können, und andererseits, welche Auswirkungen diese auf Einkommen, Arbeitsbedarf und Akzeptanz seitens der Landbevölkerung haben.

Wissensdefizite, die es zu klären gibt, sind nachfolgend aufgeführt:

- Ist eine Degradation des Bodens durch die traditionale landwirtschaftliche Nutzung eingetreten?

- Mit welchen agroforstlichen Maßnahmen lassen sich Bodenfruchtbarkeit und Erosionsschutz stabilisieren oder fördern?

- Welche Konsequenzen lassen sich daraus zur Vermeidung zukünftiger Bodendegradation folgern? Lässt sich eine Verbesserung bei Änderung der Nutzungsform erwarten?

- Welchen Beitrag liefern Erosionsschutzmaßnahmen für den Erhalt der Bodenfruchtbarkeit an den Hanglagen?

- In welchem Ausmaß führen diese Maßnahmen $\mathrm{zu}$ der gewünschten Einkommensstabilisierung, und wovon hängt die weitere Akzeptanz von agroforstlichen Maßnahmen ab?

- Wie kann ein Modellprojekt gestaltet werden, um den Bauern neue landwirtschaftliche Techniken nahe zu bringen? 


\section{NUTZUNGSSYSTEME UND BODENEROSION}

\subsection{Shifting cultivation system- traditionelle Nutzungssysteme in Nordvietnam}

Shifting cultivation oder Wanderfeldbaus spielt eine bedeutende Rolle bei den Landnutzungssystemen der Welt. Diese traditionelle Landnutzungsform gibt es in Afrika, Südamerika und Südostasien. Allein in Asien werden ca. 30\% des anbaufähigen Bodens der Welt auf diese Weise genutzt und Nahrung für ca. 8\% der Weltbevölkerung gewonnen (HATCH, 1982). Nach GEROLD (1991a) sind Länder mit niedrigem wirtschaftlichen Entwicklungsstand in den Tropen z.T. gezwungen und durch technologischen Fortschritt in der Lage, ehemals fast unberührte oder durch traditionelle Brandrodung extensiv genutzte Waldflächen zu erschließen und landwirtschaftlich zu nutzen. In den meisten tropischen Ländern erfolgte die Agrarentwicklung nach 1960 in erster Linie nach dem Prinzip der Waldrodung und Flächenausweitung sowie einer starken Verkürzung des Anbau-Brache-Zyklus beim Brandrodungsfeldbau. (GEROLD, 2002).

In Südostasien betreiben ungefähr 50 Mio. Menschen, also in etwa 6-7\% der Gesamtbevölkerung Wanderfeldbau auf einer Fläche von 100-110 Mio. ha (SPENCER, 1966). Dabei stellt shifting cultivation den Hauptfaktor für Abholzung und Bodendegradation dar (HADLEY \& LANLY, 1983 und NAIR, 1993). Aufgrund einer rasch wachsenden Weltbevölkerung, besonders in den wenig-industrialisierten Ländern, ist die Landnutzungsform des Brandrodungsfeldbaus in den letzten Jahrzehnten verstärkt in den Blickwinkel eines kritischen Interesses gerückt (WEISCHET, 1981; KELLOGG, 1962; PETER \& NEUENSCHWANDER, 1988).

In den Hügel- und Gebirgsbereichen von Vietnam wird Brandrodungsfeldbau oder slash and burn von über 5 Mio. Menschen aus 54 ethnischen Gruppen durchgeführt. Die Bereiche werden in die Nordgebirgsregion „nuong“ und in das Gebiet der zentralen Hochländer (Tay Nguyen), genannt „ray“, unterteilt. In offiziellen Dokumenten wird für diese Agrarregion die Bezeichnung ,nuong ray“ verwendet. (THAI \& DAU, 1997).

Traditionsgemäß holzen Landwirte die Waldvegetation des Hochlandes ab und legen ihre Parzellen dann per Brandrodung frei. Auf den Feldern werden dann für zwei bis drei Jahre lokale Trockenreissorten angebaut.

Der Wanderfeldbau in den Inneren Tropen, mit aus dem Abbrennen des Waldes resultierender Aschedüngung, gilt allgemein als traditionelle Form der Bodenbewirtschaftung und - bei einer 10- bis 20- jährigen Brache - auf Ferralsols und 
Acrisols als mehr oder weniger umweltverträglich (SIOLI 1984b; BRAUNS und SCHOLZ, 1997). Allerdings ist die Methode sehr flächenintensiv und erlaubt nur geringe Bevölkerungsdichten. Hinzu kommt der Mangel an Pflanzennährstoffen, der durch das Brandroden nur kurzzeitig gemindert werden kann. Kalium, Calcium und Magnesium beispielsweise werden schnell aus den Böden ausgewaschen. Gleiches gilt für andere Nähr- und Spurenelemente, denn die Adsorptionsfähigkeit der kaolinitisch geprägten Tonfraktion ist gering. Ein großer Teil des Stickstoffes verflüchtigt sich bereits beim Verbrennungsvorgang, Phosphor wie auch verschiedene Spurenelemente sind für die Pflanzen durch die feste Bindung an die Sesquioxide besonders in den sauren Ferralsols nur in geringen Mengen verfügbar. Zusätzlich wirken die hohen Aluminiumgehalte in Acrisols und Ferralsols toxisch. Bei kürzeren Brachezeiten und bei Einführung des nicht angepassten großflächigen und dauerhaften Regenfeldbaus, der aus den Savannen mehr und mehr in den Regenwälder vordringt, drohen irreversible Schäden, beispielsweise durch Bodenerosion, Decksedimente oder das Aushärten vorhandener Plinthite.

Nach NeugeBauer (1986) ist das Brandrodungsverfahren vorrangig durch den Wechsel der Feldflächen unter Einschaltung einer langdauernden Brache charakterisiert und weniger durchgängig mit einem Wohnsitzwechsel des „dem Feld folgenden Bauern“ verbunden, wie häufig angenommen wird. Die traditionelle, kleinflächige Brandrodung stellt einen eher oberflächlichen Eingriff in das ökologische Gefüge dar:

„... es erfolgt weder eine eigentliche Rodung- die Stubben werden im Boden gelassen und bilden die Grundlage für die nachfolgende Regeneration des Waldes, noch ist das Hacken des Bodens immer Bestandteil des Verfahrens... Viele Brandrodungssysteme erlauben durchaus die Sesshaftigkeit. Fällen und Brennen sind allen Verfahren gemeinsame Kulturpraktiken“ (NEUGEBAUER, 1986).

Währen die ethnischen Gruppen traditionell immer sehr kleine Flächen rodeten und abbrannten, haben in den letzten Jahren immer großflächigere Rodungen weiter Regenwaldgebiete stattgefunden, die normalerweise mit Abbrennen verbunden sind. Diese dienen kommerziellen Ausbeutungsinteressen wie Holzraubbau mit anschließender Großviehhaltung, Plantagenwirtschaft in Monokulturen usw. - Nach NEUGEBAUER (1986) werden ca. 20 ha Tropenwald weltweit pro Minute vernichtet.

Charakteristisch für das Brandrodungssystem ist, dass zwar der Energieinput, der praktisch vollständig aus menschlicher Arbeit besteht, im Vergleich zu anderen Nutzungssystemen sehr gering ist. Nach dem heutzutage um die $80 \%$ der Tropenbewohner das Brandrodungssystem paktieziren, ist in diesem Zusammenhang 
Neugebauers (1986) Hinweis, dass historische Quellen belegen, dass der Brandrodung bereits in vorspanischer Zeit Verbote auferlegt wurden, gerade in der gegenwärtigen Situation der tropischen Wälder von großer Bedeutung. Denn auch die autochthonen ethnischen Gruppen und Völker, die mit dem Markt in Berührung kommen und das kommerziell erhältliche Warenangebot kennenlernen, unterliegen notgedrungen der Tendenz, immer mehr in die cash crop - Produktion gedrängt zu werden. Die ehemals relativ naturschonenden traditionellen Brandrodungsformen werden dabei zunehmend transformiert und können dann eine wachsende Gefahr werden, die eigenen Lebensgrundlagen durch Übernutzung und Degradierung in Frage zu stellen.

\subsection{Forschungsstand über Bodenerosion}

In der Erosionsforschung ist der Boden die zentrale Ausstattungsgröße. Er fungiert gleichzeitig als Subjekt und Objekt des Erosionsgeschehens. Als Subjekt steuert er durch seine Beschaffenheit den Oberflächenabfluss und den Abtrag und als Objekt unterliegt er einem lateralen und vertikalen Stofftransfer (ROHRER, 1985).

Zusammenfassende theoretische und methodische Betrachtungen zum Forschungsstand der Bodenerosion sind die Arbeiten von RICHTER (1985), BORK (1988), GABRIELS \& BOODT (1980), BRYAN (1987), KiRKBY \& MORGAN (1980), LAL (1988), BOARDMAN et al (1990), Rorke (1990) ThORnes (1990), Morgan, (1999), Richter (Hrsg.) (1998), REHM (Hrsg.) (1986).

Der Brandrodungsfeldbau lässt sich in seinen Auswirkungen auf die Bodeneigenschaften durch nachfolgende Charakteristika beschreiben und die Ergebnisse wurden in aktuelleren Studien belegt. Nach Gerold, (1986b, 1991a; 2002); BARrow, (1991); Ross et. al, (1999); ANDRIESSER et. al, (1984; 1987a; 1987b); ANDRIESSER (1987); DRECHSEL, (1992); Thai \& NGuYen, (1997; 1999); HoAng FAgerström et al., (2000); Julio \& CASSEl, (1996); Nyle, (1996); KleinMAN et. al, (1996):

- Zerstörung der natürlichen Vegetation durch Rodung und Abbrennen führt zu Veränderungen der Nährstoffhaushalte.

- geringere Biomasse $\rightarrow$ niedrigere aktuelle Evapotranspiration $\rightarrow$ Aridisierung des Klimas.

- Bodenverhärtungen $\rightarrow$ sinkendes Infiltrationsvermögen des Bodens.

- geringere Zufuhr von pflanzlich- organischem Material.

Ein geringeres Wasserspeicherungsvermögen der Vegetation führt $\mathrm{zu}$ erhöhtem Oberflächenabfluss bzw. erhöhter Infiltration, was zu einer Verschlechterung der Wasserbilanz und zur Erhöhung des vertikalen und lateralen Nährstoffaustrags führt. Des 
Weiteren kommt es zur Zerstörung des Humusgleichgewichtes sowie zur Erhöhung der Mineralisationsraten durch stärkere Insolation und Belüftung des Oberbodens.

Außerdem steigt die Auswaschung von Huminsäuren, und austauschbaren Basen, was zu einem Sinken des gesamten Austauschkomplexes, der Kationenaustauschkapazität und der pflanzenverfügbaren Nährstoffe führt. Die Reduzierung der Kationenaustauschkapazität nach Entwaldung ist in tropischen Ökosystemen 50\% höher als in den gemäßigten Breiten.

„In vielen Arbeiten wird der Grund des Produktionsrückganges kontrovers diskutiert. Dass die Pflanzenproduktion nach der Inkulturnahme von tropischen Böden schnell absinkt, wird im Allgemeinen auf den Nährstoffentzug durch die Anbausorten, die Bodenerosion sowie das Einfallen von Unkräutern zurückgeführt" (nach: ANDRIESSE \& SCHElHAas, 1987a; 1987b; Tran, 1998; Thai \& NGUYeN, 1999). Zur Zeit laufen in den in meisten Ländern Südostasiens, wie auch im Vietnam Untersuchungen zur Erfassung der Bodenerosion. In Vietnam konzentrieren sich diese Forschungsvorhaben auf die Provinzen in der Nähe der Hauptstadt Hanoi. Über das Regenwaldgebiet oder die Bergländer Vietnams liegt bislang keine wissenschaftliche Arbeit über die Beziehung zwischen Niederschlägen und Bodenerodierbarkeit sowie zwischen Bodenverlust und Ackernutzung vor. Das Problem der Bodenerosion im Zusammenhang mit landwirtschaftlicher Nutzung ist im Hochland Vietnams nicht ausreichend erforscht.

Erst zu Beginn der 80er Jahren wurde in Vietnam auf das Problem der Bodenerosion wissenschaftlich aufmerksam gemacht. Die ersten Arbeiten konzentrieren sich auf die Bewertung der Bodenerosion im Zusammenhang mit der Anwendung neuer Agrartechniken zum Obstanbau in der Provinz Nghe An, im Hochland von Vietnam LE \& DoAn, (1985). Auch Pham ( 1990); NGUYen (1990) haben in ihren Untersuchungen über Bodenerosion bei verschiedenen landwirtschaftlichen Anbaumethoden berichtet. TON \& LE (1996) zeigen, dass im Hochland von Vietnam von 25 Mio Hektar landwirtschaftlich genutzter Fläche 13 Mio Hektar schwerwiegende Erosionsschäden aufweisen. Die Hauptursachen der Bodenerosion sind die großen Hangneigungen und die hohen Niederschläge in konzentrierter Form. Diese Autoren berichteten auch, dass auf rötlich gelben Böden mit Hangneigungen von 20-25 kein Bodenschutz betrieben wird. Der Bodenabtrag betrug dort ca. 150-170 t/ha/a. Die landwirtschaftliche Nutzfläche verringerte sich durch Bodenerosion im Nordwesten und Südwesten des Landes um 1-2\% pro Jahr. Mit dem Prozess des Bodenabtrages hat die Ferralitisierung der Böden zugenommen, wodurch die Bodenfruchtbarkeit weiter verringert wird. 
Nach BUI (1993) gingen an einigen Hangbereichen nach 2-3 jährigen Mais- oder

Trockenreisanbau der gesamte Oberboden verloren. Ohne Bodenbedeckung gingen 124 t/ha/a des Oberbodens in Northwesten des Berglands von Vietnams verloren. 119-276 t/ha/a Boden gingen in Trockenreis oder Maniok in Tay Nguyen (Süden) verloren (NGUYEN, 1994).

NGUYEN \& THAI (1999), haben Untersuchungen durchgeführt bei denen herauskam, dass der Bodenabtrag von Niederschlagshäufigkeit, Niederschlagsintensität, Hangneigung, Bodenart, Bodenbedeckung und Landnutzung abhängt. Im Wald findet dabei ein nur geringer Bodenabtrag von ca. 2-5 t/ha/a statt. Bei Grünem Tee beträgt der jährliche Abtrag 3-4 t/ha, bei Maniok oder Kulturpflanzen 40-100 t/ha/a je nach der Bodenbedeckung. Bei fehlender Bodenbedeckung ist der Bodenabtrag je nach Bodenart mit 80-100 t/ha am größten.

Ein Problem vieler Studien besteht jedoch darin, weniger den inneren Zusammenhang zwischen traditionellem Wertetesystem, Anbautechnik und ökologischen Beziehungen zu thematisieren, als Beobachtungen über den Arbeitsablauf zu berichten. Dennoch können sich aus derartigen Untersuchungen wichtige Hinweise ergeben, auf die sich die Konzeption eines intensiven, an vorhandene Traditionen anknüpfenden ökologischen Anbaus mit stützen kann.

Diese Literaturübersicht über Bodenerosionsuntersuchungen lässt erkennen, dass sich die Untersuchung bisher in Vietnams auf einige Provinzen und Regionen in de Nähe von Hanoi im Süden beschränken. Tatsächlich sind in Bergregion kaum quantitative Untersuchung über die Bodenerosion durchgeführt worden.

\subsection{Bedeutung von Erosion und Erosionsschutzmaßnahmen}

Unter dem Begriff Erosion versteht man die Verlagerung von Bodenmaterial entlang der Oberfläche. Man unterscheidet zwischen Wasser- und Winderosion. Wassererosion tritt auf hängigem, abschüssigem Gelände mit geringer Bodenbedeckung und konzentrierten, hohen Niederschlagsmengen auf. Als Ursachen für geringe Bodenbedeckung sind die Beseitigung von Wäldern, Büschen und Wiesen, destruktive Anbautechniken und Anbaupraktiken auf marginalen Standorten, die Reduzierung der bodenbedeckenden Anbauphase und Naturbrache sowie Überweidung der Flächen zu sehen (REINING, 1992). Winderosion tritt verstärkt auf Flächen mit geringer Bodenbedeckung bei gleichzeitig hohen Windgeschwindigkeiten auf. Die Wirkung der Winde wird durch die Beseitigung von Windbrechern wie Wald, Busch und Einzelbäumen verstärkt. 
In den Untersuchungsgebieten in den Bergregionen Nordvietnams werden Flächen mit extremer Hangneigung landwirtschaftlich genutzt. Der Vorgang der Wassererosion ist so von besonderem Interesse.

Der Bodenabtrag durch Wasser läuft in zwei Schritten ab. Im ersten Schritt werden die Bodenaggregate durch die beim Aufprall der Regentropfen übertragene kinetische Energie zerschlagen. Es entsteht transportierbares Feinmaterial. Im zweiten Schritt kommt es zum Abfluss dieses und bereits vorhandenen Feinmaterials mit dem Oberflächenwasser hangabwärts. Während des Wasserabflusses werden weitere Bodenpartikel losgelöst und abtransportiert. Lässt die Schleppkraft des Wassers nach, werden die Bodenpartikel abgelagert. Des Weiteren kann es durch den Aufprall der Regentropfen zu einer Versiegelung der obersten Millimeter des Bodens kommen. Ton und Schluffteilchen setzen sich in den Bodenporen fest und führen zu deren Verstopfung. Als Folge sinkt die Infiltrationsrate und der Oberflächenabfluss nimmt zu, was zu vermehrtem Bodenabtrag führt. Bei den Abtragsformen unterscheidet man, je nach Ausprägung, zwischen Flächen-, Rillen- und Graben- bzw. Tunnelerosion (SCHEFFER. et al, 1992).

Um den Bodenabtrag quantitativ erfassen bzw. abschätzen zu können, ist es erforderlich, die natürlichen Faktoren Regen- und Oberflächenabfluss, Bodenerodierbarkeit, Hanglänge und Hangneigung sowie die anthropogenen Faktoren Bodenbedeckung, Bodenbearbeitung und Erosionsschutz- und Flächenmanagement, die den Abtrag im wesentlichen bestimmen, zu berücksichtigen. WISCHMEIER und SMITH (1978) stellten die allgemeine Bodenabtragsgleichung, die Universal Soil Loss Equation (USLE) auf, mittels derer die Schätzung des langfristigen mittleren jährlichen Bodenabtrags möglich ist. Die Gleichung lautet:

$$
A=K^{*} R^{*} L^{*} S * C * P
$$

Mit

$\mathrm{A}=$ Langjähriger, mittlerer jährlicher Bodenabtrag in $\mathrm{t} / \mathrm{ha}$

$\mathrm{K}=$ Bodenerodierbarkeitsfaktor. Er ist ein Maß für die Erosionsanfälligkeit eines Bodens und wird von dessen nachstehenden Eigenschaften bestimmt:

Gehalt an Schluff und Feinstsand (0,002-0,1 mm)

Gehalt an Sand abzüglich des Feinstsandes (0,1-2mm) (in der Acker krume)

Gehalt an organischer Substanz Aggregatklasse 
Durchlässigkeitsklasse (des ganzen Bodenprofils)

$\mathrm{R}=$ Regenerosivitätsfaktor. Er kennzeichnet die gebietsspezifische Erosionskraft der Niederschläge und wird aus der kinetischen Energie und der Niederschlagsintensität aller erosionswirksamen Einzelregeln während des Jahres berechnet.

$\mathrm{L}=$ Hanglängenfaktor. $\mathrm{Zu}$ berücksichtigen ist nur die erosive Hanglänge, d.h. der Abstand zwischen der Stelle am Hang, wo der Oberflächenabfluss einsetzt und der Stelle, wo die Sedimentation beginnt bzw. wo das Wasser in Gräben abgeleitet wird.

$\mathrm{S}=$ Hangneigungsfaktor. Er bringt das Gefälle der Hangfläche (in \%) zum Ausdruck.

$\mathrm{C}=$ lokalen Bodenbedeckungs- und Bodenbearbeitungsfaktor. Er wird bestimmt, indem der Relative Bodenabtrag (RBA) zur relativen Verteilung des erosiven Niederschlags über das Jahr in Bezug gebracht wird. Im RBA wird die Erosionsanfälligkeit in Abhängigkeit von der Vegetationsbedeckung und des Oberbodenzustandes zu jedem Zeitpunkt der Kultur als Verhältnis (in \%) zu einer gleichen Fläche unter Schwarzbrache $(=100 \%)$ ausgedrückt.

$\mathrm{P}=$ Erosionsschutz- und Flächenmanagementfaktor. In ihm werden die Schutzwirkung von Kontur- und Streifenanbau quantifiziert. Terrassierungsmaßnahmen sowie das Anlegen von Wällen, Dämmen und Gräben quer zur Hangrichtung gehen sowohl in den L- Faktor (Hangverkürzung) als auch in den P- Faktor (Konturnutzung) ein.

Um die Bodenabträge auf den Parzellen der Kleinbauern mit Hilfe der Abtragsgleichung abschätzen zu können, wäre es unabdingbar, den besonderen Bedingungen Rechnung zu tragen. Das hieße vor allem die Berücksichtigung

- des Anbaus traditioneller Kulturpflanzen,

- traditioneller bzw. alternativer Anbausysteme (z.B: typische Fruchtfolgen, Mischanbau, agroforstlicher Anbau),

- des Pflanzenbaus in unterschiedlichen Höhenstufen (verschieden lange Vegetationsperioden der gleichen Kultur),

- der traditionellen Bodenbearbeitung (Hacke, Hakenpflug, etc.) bei der Ermittlung des C- Faktors.

Es sei noch darauf hingewiesen, dass, sofern die Faktorwerte für den betreffenden Standort bekannt sind, mit Hilfe der ABAG (Allgemeine Boden-Abtrags- Gleichung) bzw. (USLE) auch abgeschätzt werden kann, inwieweit sich Maßnahmen wie Hangverkürzung, Fruchtwechsel, Konturanbau, etc. mindernd auf den Bodenabtrag auswirken würden bzw. welche der realisierbaren Maßnahmen ergriffen werden müsste, um den zu erwartenden Abtrag unter die Toleranzgrenze zu senken. 


\subsection{Verbesserte Bodennutzungssysteme}

Im Vordergrund des Erosionsschutzes in Vietnam sollte eine standortgerechte Landnutzung stehen, um den anthropogen bedingten Bodenabtrag so weit wie möglich einzuschränken und die Bodenfruchtbarkeit zu erhalten. Darüber hinaus notwendig werdender Erosionsschutz muss auch technische Maßnahmen miteinbeziehen.

\subsubsection{Fruchtwechsel}

„Jeder Bauer muss sich an die klar erkannten Gesetzmäßigkeiten des Fruchtwechsels halten, verstößt er gegen sie, so sind wirtschaftliche Fehlschläge die Folge“ (BECKER, 1994)

Eine sinnvolle Fruchtfolgegestaltung wirkt dem Bodenabtrag durch Wasser entgegen und wird daher auch für die Landwirtschaft als Erosionsschutzmaßnahme empfohlen Ein quantitativer Vergleich von Monokulturanbau und verschiedenen Fruchtfolgen hinsichtlich ihrer Auswirkung auf das Ausmaß der Wassererosion kann anhand des CFaktors der ABAG abgeschätzt werden.

Die Bodenschutzwirkung einer Fruchtfolge im Vergleich zur Monokultur erosionsfördernder Feldfrüchte (Mais, Maniok, Reis) steigt mit dem Bedeckungsgrad und seiner zeitlichen Ausdehnung sowie mit der Menge an Ernterückständen. Hinzu kommen:

- $\quad$ die Durchwurzelungsintensität und - tiefe.

- $\quad$ die Pflanzendichte (Stengel $/ \mathrm{m}^{2}$ ).

Als für den Raum geeignete Fruchtfolgen von Rein- und Mischbeständen werden genannt:

- $\quad$ Mais, Bohnen, Gemüse.

- $\quad$ Süßkartoffeln, Mais, Tomaten.

- $\quad$ Mais, Reis, Süßkartoffeln.

Eine speziell dem Erosionsschutz dienende Variante der Fruchtfolgegestaltung stellt der Streifenanbau dar. Der Streifenanbau ist gekennzeichnet durch gleichzeitigen Anbau von erosionsfördernden (Mais, Süßkartoffel) und erosionshemmenden (Getreide, Ackerfutter) Früchten in sich abwechselnden, isophypsenparallelen Streifen (SCHWERTMANN, 1987). 


\subsubsection{Mischanbau}

Unter dem Begriff „Mischanbau“ soll hier jeglicher Anbau von zwei oder mehreren, sich gegenseitig beeinflussenden Feldfrüchten, die während eines Teiles oder während ihrer gesamten Vegetationsperiode gemeinsam auf derselben Fläche wachsen, verstanden werden. Auch Pflanzenbestände, die in erster Linie der Futtergewinnung, der Gründüngung oder der Bodenbedeckung dienen, gelten in diesem Zusammenhang als Feldfrüchte.

Hinsichtlich des Bodenschutzes liegt der Vorteil des Mischanbaus gegenüber der Reinkultur vorrangig in der dichteren Vegetationsdecke sowie in der zeitlichen Ausdehnung derselben, wodurch der Boden vor Verschlämmung und Erosion bewahrt wird (Bergeret, 1984; KotsChi et al, 1989; KUYPERS et al, 1987; PRINZ, 1986).

Nach CAesar (1986) zit. In (KRetzschmar, 1990) setzt die Schutzwirkung ab 35\% Flächenbedeckung ein und wird ab 70\% nahezu vollkommen. Außerdem verstärken eine erhöhte Biomasseproduktion KUYPERS (1987), eine intensive Durchwurzelung (MÜLLERSÄMANN, 1986) sowie eine verminderte Bodenbearbeitung (ELLENBERG, 1984) den Schutzeffekt von gemischten Kulturpflanzenbeständen. Er kann im C-Factor der ABAG abgeschätzt werden.

\subsubsection{Agroforstliche Systeme (Agroforstwirschaft)}

Möglicherweise bietet die moderne Agroforstwirtschaft, ein Mischkultursystem aus Gehölz- und annuellen Nutzpflanzen, eine wirtschaftlich erfolgreiche und ökologisch stabile Alternative (ScholZ, 1984; DopPler, 1991; NAIR \& MUSChLER, 1993). Dabei führen die höheren Bäume durch ihre Streu immer wieder Nährstoffe in den Boden zurück, wo die Kulturpflanzen sie aufnehmen. Limitierend wirken der Schattenwurf der hohen Bäume sowie die Konkurrenz zwischen den Gehölzwurzeln und den Nutzpflanzen um Wasser und Nährstoffe (ZECH, 1997). Allerdings können auch Agroforstsysteme, ähnlich wie andere permanente stockwerkartige Anbaumethoden, einen schleichenden Nährstoffverlust durch Bodenabtrag und Biomasseentnahme nicht verhindern. Düngergaben sind auch hier auf längere Sicht notwendig (KÖNIG, 1996).

„Agroforstwirtschaft ist ein Sammelbegriff für viele verschiedene Landnutzungssysteme und Technologien, bei denen ausdauernde Holzgewächse planmäßig auf der gleichen Fläche wie landwirtschaftliche Pflanzen oder Tiere bewirtschaftet werden. Die Kombination der forst-, land- und weidewirtschaftlichen Strukturelemente und 
Maßnahmen kann dabei sowohl räumlich als auch zeitlich erfolgen“. MAYDELL (1986) setzt dabei voraus, dass sich Bäume und Sträucher, Kulturpflanzen und Tiere als Komponenten eines Gesamtsystems deutlich nachweisbar gegenseitig beeinflussen.

Hinsichtlich des Schutzes vor Wassererosion auf geneigten Ackerflächen wird insbesondere zwei agroforstlichen Systemvarianten Bedeutung beigemessen:

Alleeanbau bzw. Hecken als Abflusshindernisse (alley cropping, hedgerow intercropping) (Kotschi et al. 1989; LAL, 1991; LoRENZ, 1990; PrinZ, 1986; TANCIO, 1988, 1991; YounG, 1989a, 1989b; YounG, 1990)

- Holzgewächse kombiniert mit technischen Erosionsschutzeinrichtungen. (EGGER, 1989; YounG, 1989a, 1989b, 1990)

Im Alleeanbau werden in bestimmten Abständen Reihen von Bäumen oder Sträuchern entlang der Isohypsen gepflanzt. In den dazwischen liegenden Alleen werden Feldkulturen angebaut. Daraus resultierende Schutzfunktionen können sein:

- $\quad$ verbesserte Bodenbedeckung, wenn das beim periodischen Schneiden der Hecken anfallende Material (Blätter u. feine Äste) zum Mulchen der Alleenflächen verwendet wird.

- $\quad$ erhöhter Widerstand des Bodens gegen die Erosionskräfte des Wassers durch Erhaltung bzw. Erhöhung des Humusgehaltes.

- $\quad$ Bildung von induzierten Terrassen durch Ablagerung und Akkumulation von Sedimenten oberhalb der Hecken (YounG, 1989a, 1990).

Holzgewächse kombiniert mit technischen Erosionsschutzeinrichtungen. (EGGER, 1989; YounG, 1989a, 1989b, 1990)

Die Arten der Bäume und Sträucher: THAI \& NGUYEN, (1998); HoANG FragERSTRÖM (2000), und TACIO (1988) empfehlen, verschiedene Spezies zu pflanzen, um der Gefahr von Krankheits- und Schädlingsbefall zu begegnen. Außerdem ergeben sich vielseitigere Nutzungsmöglichkeiten (z.B: Mulch, Futter, Brenn- und Bauholz, Früchte, etc.). NGUYEN \& Do (1998) haben Untersuchungen in Thainguyen durchgeführt bei denen herauskam, dass die geeigneten Gründüngungspflanzen in Bergland von Vietnam Tephrosia cadida, Flemingia congesta, Leuceana glauca, Desmodium renzoni sind Diese Pflanzen haben die Fähigkeit sich schnell zu regenerieren. Damit können sie 3-4-mal im Jahr geschnitten 
werden. Die Erträge der geschnittenen Pflanzen erreichen 6-7t/ha/a (s.Tab.1) mit einer hohen Bodenbedeckung in der Regenzeit.

Tab. 1: Ertrag der geschnittenen Pflanzen im Jahr (t/ha)

\begin{tabular}{lrr}
\hline Pflanzenarten & 1. Jahr & 2.Jahr \\
\hline Tephrosia cadida & 7,3 & 7,4 \\
\hline Desmodium renzoni & 6,6 & 7,0 \\
\hline Flemingia congesta & 5,9 & 7,3 \\
\hline Leuceana glauca & 4,8 & 5,6 \\
\hline
\end{tabular}

Quellen: NGUYEN \& Do (1998)

Gründüngungspflanzen sollten möglichst viele der folgenden Eigenschaften aufweisen:

Von den genetischen Eigenschaften her sollten sie eine hohe Biomasseproduktion in relativ kurzer Zeit erbringen und den Boden schnell bedecken.

- die am Standort dominanten bzw. schädlichen Unkräuter kontrollieren und unterdrücken.

- eine hohe Blattproduktion und relativ geringe Anteile schnell verholzender Stengel liefern.

- möglichst tief oder intensiv wurzeln

- ein hohes Nährstoffaufschlussvermögen besitzen.

- N-fixierende Eigenschaften haben (Leguminosen).

- eine hohe Affinität zu Mykorrhira haben, um das Inokulum im Boden zu verbessern.

Vom ackerbaulichen Standpunkt ist zu fordern, dass

- der Anbau relativ wenig Arbeit erfordert.

- das Saatgut billig ist und einfach - möglichst am Ort - erzeugt werden kann (Verfügbarkeit).

- die Gründüngungspflanze kontrollierbar ist und bleibt und nicht außer Kontrolle gerät

- die Gründüngungspflanze auch eine Nutzung als Futterpflanze erlaubt oder sonst eine Nebenfunktion erfüllt.

- Abstand der Holzgewächse in der Reihe

Young (1990), Thai \& NGuYen (1998) hält einen Pflanzabstand von $25 \mathrm{~cm}$ bei einreihigen und bis zu $50 \mathrm{~cm}$ bei zwei- oder mehrreihigen Hecken für angebracht. Da die Hecken in erster Linie zum Zwecke des Erosionsschutzes angelegt werden sind sicherlich möglichst enge Pflanzenabstände zu bevorzugen. Auf diese Weise entsteht frühzeitig eine weitgehend undurchlässige Barriere bzw. es lässt sich eine möglichst hohe 
Biomasseproduktion, in Form von Blättern und feinen Zweigen (Mulchmaterial) erzielen. Letzteres kann entscheidend für den Erfolg des Alleenanbaus sein, da der Erosionsschutzwirkung durch Bodenbedeckung ( $>60 \%$ ) zumindest die gleiche Bedeutung beizumessen ist wie der Barrierefunktion der Hecken (YounG, 1989a, 1990)

- Breite der Hecken

HoAng Ha Ström, Thai (1998); Young (1990) zufolge sind Versuche mit breiten Hecken gemacht worden, wobei die einreihige Hecken diejenige ist, welche am häufigsten als Erosionsschutz angewendet wird. Während letztere weniger landwirtschaftliche Nutzfläche einnimmt, kann bei hohen Oberflächenabflussrate eine mehrreihige Hecke angebracht sein. Abgesehen von der natürlichen Streu verlässt allerdings die gesamte Biomasseproduktion der Hecken die Pazelle in Form von Futter bzw. Brenn- und Nutzholz, d.h. der in den Alleen angebaute Mais wird nicht gemulcht. Teilweise sedimentieren die im oberen Bereich der $8 \mathrm{~m}$ breiten Alleenflächen abgetragenen Bodenartikel im unteren Bereich, wodurch Ansätze der Terrassenbildung sichtbar werden.

Die Entfernung zwischen den Hecken sollte 6-8m betragen (NGUYEN \& LAN,1998). Diese Hecken können 3-4 mal im Jahr geschhnitten werden. Das Schnittmaterial kann als Mulch verwendet werden. Auch wird durch die Heckenreihen nach einigen Jahren eine dichte Barriere gegen Bodenerosion aufgebaut. Die Vorteile des höheren Schnittes besteht darin, dass Holz als Nebenprodukt anfällt und dass sich ein tiefergehendes Wurzelwerk entwickelt, das mit den Feldpflanzen in geringerem Maße um Wasser und Nährstoffe konkurriert. (TACIO, 1988; YounG, 1990)

In Bezug auf den Erosionsschutz gibt es zwei Alternativen der Verwendung des Schnittmaterials:

- Ausbringung als Mulchdecke.

- Auslegen des Schnittmaterials entlang der Hecken, um die Barrierewirkung zu verbessern.

Betont werden muss, dass jegliches Entfernen von Biomasse als Futter bzw. Brenn- oder Nutzholz die Erosionsschutzwirkung der Hecken mindert. Ist die Futternutzung des Blattmaterials unabdingbar, so sollte zumindest ein gewisser Ausgleich durch Rückfuhr von Biomasse in Form von Mistdüngung angestrebt werden. 


\subsubsection{Mulchen}

Unter Mulchen versteht man die Bedeckung des Bodens mit Pflanzenzückständen wie Stroh, Maisstängeln, Palmwedeln oder stehenden Stoppeln. Besonders wirksam ist Mulch im Vergleich zum gleichen Bedeckungsgrad durch einen Pflanzenbestand dadurch, dass er direkt der Bodenoberfläche aufliegt und somit die Fallgeschwindigkeit der Tropfen aufgrund der äußerst geringen Fallhöhe auf ein Minimum reduziert. (SCHWERTMANN et al, 1987)

Die Mulchschicht bremst die Geschwindigkeit des Oberflächenabflusses (MÜLLERSÄMANN, 1986), in besonderer Weise, wenn sie durch konturpallele Pflanzenreihen stabilisiert wird. Folglich verringern sich die Schubspannung des Wassers und damit der Bodentransport.

Nach LAL (1976) liegt der größte Vorteil einer solchen Kulturtechnik auch für die tropischen Böden in der,, sicheren und billigen Minderung der Erosion“. Die Versuchergebnisse von LAL (1976) zeigen, dass durch Mulchraten von nur 2 t/ha der Oberflächenabfluss um $60 \%$ und die Bodenerosion um durchschnittlich über $90 \%$ verringert werden konnte.

Nach DERPSCH et. al. (32) gewährleisten 4-6 t Mulchmaterial pro Hektar eine hundertprozentige Bodenbedeckung. MORGAN (1980) erachtet eine Strohmenge von $5 \mathrm{t}$ ha, was einem Bedeckungsgrad von ca. 70\% entspricht, als notwendig, um einen ausreichenden Erosionsschutz zu erzielen.

Außerdem hat Mulch eine positive Wirkung auf die Ertragsbildung. MÜLLER- SÄMANN (1986) zitiert Ergebnisse von FRANKE (1981), der sogar Ertragszunahmen um das 3- bis 6 Fache (je nach Sorte) erzielen konnte.

\subsection{Ursachen der Erosion in Vietnam}

Als Ursachen für Erosion sind in Vietnam vor allem die Armut der ländlichen Bevölkerung, der Bevölkerungsdruck, begrenzte institutionelle Kapazitäten sowie eine nur langsam fortschreitende Allokation der Landnutzungsrechte zu sehen (UNDP, 1996). In einigen Teilen Vietnams, vor allem in den ländlichen Regionen, liegt das Pro- KopfEinkommen pro Jahr unter 100 USD. Faktoren, die mit dieser Armut in Verbindung stehen und die Ausbeutung des Bodens zur Existenzsicherung verstärken, sind chronischer Brennholzmangel, geringe Nahrungsmittelverfügbarkeit, hohe Unterbeschäftigung und eine hohe Arbeitslosenquote. 
Die Bevölkerungsdichte, bezogen auf die landwirtschaftliche Nutzfläche, beträgt 500 Personen $/ \mathrm{km}^{2}$, in sehr intensiv genutzten Regionen, wie zum Beispiel dem Red River Delta, sogar mehr als 1000 Personen $/ \mathrm{km}^{2}$. Zudem ist die Fruchtbarkeitsrate der ländlichen Bevölkerung relativ hoch. Es wird geschätzt, dass sich die Bevölkerung in den nächsten 40 Jahren auf annähernd 140 Mio. Menschen verdoppeln wird. Die Konsequenz des Bevölkerungsdrucks ist, dass die Flächengröße, die einer Person zur landwirtschaftlichen Nutzung zur Verfügung steht, durchschnittlich zur Zeit nur 0,1 ha beträgt. Folgen sind unter anderem Migrationen in marginale Gebiete und Nutzung marginaler Flächen, die für die landwirtschaftliche Nutzung ungeeignet sind. (FAO; 1996)

Die langsame Verteilung von Landnutzungsrechten führt zu immer noch ungeklärten Besitzverhältnissen landwirtschaftlicher und forstwirtschaftlicher Fläche. Das Fehlen langfristig gesicherter Nutzungsrechte vermindert die Bereitschaft, langfristig wirksame Investitionen auf den Betriebsflächen durchzuführen. Maßnahmen zur Verminderung des Bodenabtrags, deren optimaler Wirkungsgrad erst nach Jahren eintritt bzw. zu „sunk costs“ führt, werden nur begrenzt durchgeführt (FAO, 1996)

Darüber hinaus herrschte über einen langen Zeitraum hinweg Krieg. Die Bevölkerung stieg durchschnittlich um 2,7\%/Jahr, von 35 Mio. Menschen im Jahre 1945 auf 65 Mio. Menschen im Jahre 1981. Um die Ernährung der Bevölkerung zu sichern, wurden große Waldflächen zugunsten der Landwirtschaft zerstört. Ein Teil des Waldes fiel 13 Mio. $\mathrm{t}$ Bomben und ein anderer Teil 72 Mio. t Gift zum Opfer. (PHAN, 2001, S54, 55)

Weitere Waldressourcen wurden durch Brenn- und Nutzholzentnahme zerstört, da ca. 90\% der Energie privater Haushalte aus pflanzlichen Brennstoffen gewonnen wird. Jedes Jahr werden 21 Million t Brennholz aus den Wäldern nutzbar gemacht.

Nach GLASOD 2000 ist Hauptursache für Bodenerosion in Vietnam die Abholzung (s. Karte 1 ) 
Karte 1: Wichtigste unmittelbare Erosionsursache nach mapping unit

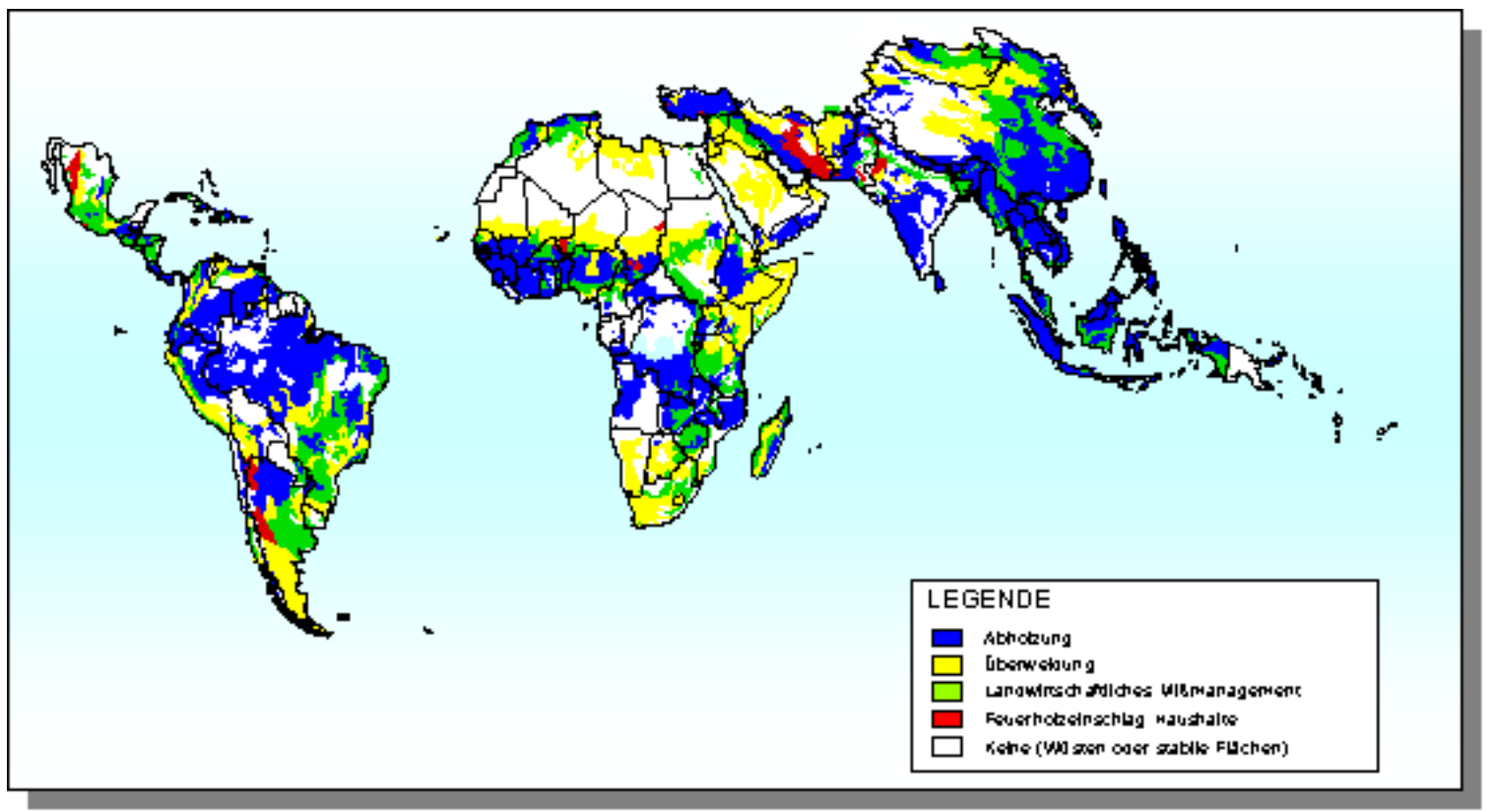

Quelle: GLASOD 2000

Eine weitere Ursache der Reduzierung der Waldflächen liegt im schon erwähnten traditionellen Wanderfeldbau mit Brandrodung, der zur Zeit von etwa 9 Mio. Menschen betrieben wird. Mit Blick auf die schnell wachsende Bevölkerung ist der traditionelle Wanderfeldbau die wichtigste Ursache für Waldzerstörung, Bodenerosion und Bodendegradation. In Folge der genannten Ursachen ist sogar bereits eine Fläche von 5,5 Mio. ha von sehr schwerwiegenden Bodendegradationen betroffen. 


\section{CHARAKTERISIERUNG DES UNTERSUCHUNGS- GEBIETES}

Ziel von Kapitel 3 ist es, umfassende Hintergrundinformationen über Vietnam sowie das Untersuchungsgebiet Babe zu geben, die es ermöglichen, die bestehende Problematik unter den gegebenen Rahmenbedingungen zu sehen und zu verstehen. Nationale und regionale geographische, klimatische, legislative und sozioökonomische Indikatoren werden dazu herangezogen

\subsection{Vietnam}

\subsubsection{Geographische Lage}

Vietnam liegt zwischen $8^{\circ}$ und $23^{\circ}$ nördlicher Breite sowie $102^{\circ}$ und $109^{\circ}$ östlicher Länge. Der Staat erstreckt sich über $1600 \mathrm{~km}$ in langgezogener S-Form entlang der Ostküste von Südostasien. Im Norden grenzt er an China und im Westen an Laos und Kambodscha. Die Außengrenze des Landes wird von der Küstenlinie am Golf von Siam und am Südchinesischen Meer über eine Länge von 3260 km gebildet.

\subsubsection{Demographische Kennzahlen}

Vietnam hat eine Fläche von $332000 \mathrm{~km}^{2}$, auf der im Jahre 2000 76,55 Mio. Menschen lebten. Das durchschnittliche Bevölkerungswachstum betrug in den Jahren 1995-2000 jährlich 2,1\%. Die Lebenserwartung eines Vietnamesen liegt bei etwa 68 Jahren. Mit 6\% weist Vietnam, verglichen mit anderen Staaten, eine verhältnismäßig niedrige Analphabetenrate auf (WeltBAnK, 1997).

Die Bedeutung des landwirtschaftlichen Sektors: Das Bruttosozialprodukt pro Kopf belief sich im Jahre 1995 auf 240 USD. 1993 lag die Einkommensverteilung nach dem GiniIndex bei 35,7. Aufgeteilt nach Sektoren erwirtschaftete der landwirtschaftliche Sektor 27\%, der industrielle Sektor $31 \%$ und der Dienstleitungssektor $42 \%$ des Bruttosozialproduktes. Die Bedeutung des landwirtschaftlichen Sektors wird deutlicher wenn man bedenkt, dass 72\% der Arbeitskräfte in diesem Sektor arbeiten und 79\% der Bevölkerung in ländlichen Regionen leben. Jeweils 14\% der Arbeitskräfte finden im Industriesektor bzw. Dienstleistungssektor Beschäftigung. 


\subsubsection{Situation Boden und Landnutzung}

Wie aus Tabelle Anhang 1 ersichtlich, zählt Vietnam weltweit zu den Ländern, die besonders hohe Anteile schwer und extrem erodierter Fläche aufweisen.

In Tab. 2 und 3 ist Vietnam im Vergleich mit anderen asiatischen Ländern dargestellt.

Tab 2: Bodendegradation in einigen asiatischen Ländern (in 1000 ha) (FAO 1995)

\begin{tabular}{|c|c|c|c|c|c|}
\hline \multirow[t]{2}{*}{ Länder } & \multirow[t]{2}{*}{ Landesfläche } & \multicolumn{2}{|l|}{ Ackerbau } & \multicolumn{2}{|c|}{ Degradierte Fläche } \\
\hline & & Fläche & $\begin{array}{l}\text { \% der } \\
\text { Gesamtfläche }\end{array}$ & Fläche & $\begin{array}{l}\% \text { der } \\
\text { Gesamtfläche }\end{array}$ \\
\hline Bangladesh & 13.017 & 9.292 & 71 & 989 & 7,4 \\
\hline China & 932.641 & 96.115 & 10 & 280.000 & 30,0 \\
\hline Indien & 297.319 & 169.990 & 57 & 148.100 & 49,8 \\
\hline Indonesien & 177.160 & 21.260 & 12 & 43.000 & 24,0 \\
\hline Laos & 23.080 & 901 & 4 & 8.100 & 35,0 \\
\hline Myanmar & 65.754 & 10.034 & 15 & 210 & 3,2 \\
\hline Pakistan & 77.088 & 20.730 & 27 & 15.500 & 17,3 \\
\hline Philippinen & 29.817 & 7.970 & 27 & 5.000 & 16,8 \\
\hline Sri Lanka & 6.463 & 1.901 & 29 & 700 & 10,8 \\
\hline Thailand & 51.089 & 22.126 & 43 & 17.200 & 33,7 \\
\hline Vietnam & $\underline{32.549}$ & $\underline{6.600}$ & $\underline{20}$ & $\underline{15.300}$ & $\underline{48,9}$ \\
\hline
\end{tabular}

Tab. 3: Länder in Asien mit hohem Anteil schwer bis extrem erodierter Flächen nach Region

\begin{tabular}{|c|c|c|c|}
\hline \multirow[t]{2}{*}{ Asien } & \multirow{2}{*}{$\begin{array}{l}\text { Bodenerosion } \\
\text { stark bis } \\
\text { extrem } \\
\text { schwer } \\
\text { ( \% der } \\
\text { Landes- } \\
\left.\text { fläche }{ }^{2}\right)\end{array}$} & \multirow{2}{*}{$\begin{array}{c}\text { Davon durch } \\
\text { Wassererosion } \\
\text { (\% der stark bis } \\
\text { extrem erodierten } \\
\text { Fläche) }\end{array}$} & Unmittelbare Ursachen $^{3}$ \\
\hline & & & $\begin{array}{l}\text { Landwitschaft Abholzung Überweidung } \begin{array}{c}\text { Holzeinschlag } \\
\text { Missmanagement }\end{array} \\
\text { „a“" „f“ } \\
\text { (\% der stark bis extrem erodierten Fläche) }\end{array}$ \\
\hline Thailand & 28 & 100 & 100 \\
\hline Vietnam & 19 & 100 & 90 \\
\hline Türkei & 17 & 100 & 100 \\
\hline Sri Lanka & 13 & 100 & 100 \\
\hline Philippinen & 9 & 100 & 90 \\
\hline Kambodscha & 9 & 100 & 100 \\
\hline \multicolumn{4}{|c|}{$\begin{array}{l}\text { Quelle: GLASOD } 2000 \\
\text { 1) Entsprechend den GLASOD-Schweregraden schwer (3) bis extrem (4) degradiert (WEC3_4) } \\
\text { 2) Landesfläche bezeichnet hier die Referenzfläche, d.h. die Landesfläche abzüglich der wastelands } \\
\text { 3) Entsprechend den im GLASOD definierten unmittelbaren Erosionsursachen (a, g, f, e) } \\
\text { Die in einem Land dominierende(n) unmittelbare(n) Erosionsursache(n) ist (sind) grau unterlegt }\end{array}$} \\
\hline
\end{tabular}




\section{Schweregrad Definition}

\begin{tabular}{|c|c|c|}
\hline$" 1^{*}$ & Gering & $\begin{array}{l}\text { Die landwirtschaftliche Eignung des Bodens ist gering fügig zurückgegangen. } \\
\text { Wiederherste llung der ursprünglichen Produktivität ist durch Verände rungen des } \\
\text { Manage me nts möglich. Die ursprünglichen biotischen Funktionen des Bodens sind } \\
\text { weitge he nd intakt. }\end{array}$ \\
\hline$n^{2 \epsilon}$ & Mittel & $\begin{array}{l}\text { Die landwirtschaftliche Produktivität des Bodens ist stark zurückgegangen, de nnoch ist } \\
\text { er weiterhin ge eignet für lokale Lardnutzungssyste re. Zur Wie derherstellung der } \\
\text { ursprünglichen Produktivität sind erheblic he Verbe sse rungen notwe ndig. Die } \\
\text { ursprünglichen biotischen Funktionen des Bodens sind teilwe ise zerstört. }\end{array}$ \\
\hline, $3^{*}$ & Schwer & $\begin{array}{l}\text { Der Boden ist auf Betriebsebene nicht mehr kulturfähg. Erheblic he bautechnische Maß } \\
\text { nahmen sind notwendig, um das Terrain wiederhe rzustellen. Die ursprünglichen } \\
\text { biotischen Funktionen des Bodens sind weitgehend ze rstört. }\end{array}$ \\
\hline$" 4^{4 \epsilon}$ & Extrem & $\begin{array}{l}\text { Der Boden ist nicht me hr kulturfähig, das Terrain kann nicht wiederhergestellt werden. } \\
\text { Die ursprünglichen biotischen Funktionen des Bodens sind vollkommen ze rstört. }\end{array}$ \\
\hline
\end{tabular}

Nach FAO (Tab.1) nimmt der Ackerbau in Vietnam im Jahre 1995 nur 20\% der Gesamtfläche ein, dagegen sind aber bereits 48,9 \% der Gesamtfläche degradiert. Das ist mehr als das Doppelte der ackerbaulich genutzten Fläche und fast die Hälfte der Fläche Vietnams. Nach einer Studie von GLASOD aus 2000 (Tab. 2) sind 19\% der Landesfläche sogar stark bis extrem stark durch Bodenerosion geschädigt. Das heißt, dass fast ein 1/5 der Fläche Vietnams nur durch erhebliche bautechnische Maßnahmen oder garnicht mehr kulturfähig ist. Verursacher ist dabei laut zu Studie zu 100 \% Wassererosion.

Die landwirtschaftlichen Anbausysteme Vietnams sind sehr komplex. Auf $85 \%$ der landwirtschaftlich genutzten Fläche wird Reis angebaut. Mais wird auf 7\% der Fläche, Süßkartoffeln auf 3\% und Kartoffeln auf 2\% der Fläche angebaut. Der typische Haushalt bewirtschaftet einen Hausgarten von 150-200 $\mathrm{m}^{2}$, in dem Mischanbau praktiziert wird. Hauptsächlich werden im Mischanbau die Kulturen Zitrone, Orange, Jackfrucht, Longan, Litschi, Guave, Banane sowie Gemüse und Futterpflanzen angebaut. In der Tierproduktion werden hauptsächlich Schweine, Büffel, Rinder, Enten und Hühner gehalten. Oft sind die Betriebe mit Fischteichen ausgestattet (FAO, 1996).

\subsubsection{Reformen im landwirtschaftlichen Sektor}

Die Demokratische Republik Vietnam hatte nach ihrer Entstehung 1954 ihre Wirtschaft in Anlehnung an die Planwirtschaft sowjetischen Stils entwickelt. Ihre wesentlichen Elemente waren eine schnelle Industrialisierung durch Staatsbetriebe, die Kollektivierung der Landwirtschaft und eine stark zentralistische Wirtschaftsplanung und -führung. Das Produktionsvolumen der landwirtschaftlichen Kooperativen richtete sich nach den quantitativen Zielvorgaben des Plans. Die Zuweisung der dafür benötigten Ressourcen erfolgte ebenfalls zentral. Vor Beginn der Reformen in der Landwirtschaft 1979 konnten 
nur 5\% der kooperativ bewirtschafteten Flächen von Familien für den privaten Gebrauch bestellt werden. Für den freien Markt war die Produktion offiziell verboten. Die Einführung eines Produktionsvertrages oder Kontraktsystems innerhalb einer Kooperative bildete die erste Phase der Reform in der Landwirtschaft. Mitglieder wurden so in die Lage versetzt auf einer eigenen landwirtschaftlichen Nutzfläche, nach Erfüllung ihrer Abgabepflicht an die Kooperative, die erwirtschafteten Überschüsse auf dem freien Markt zu verkaufen. Gleichzeitig wurden die Landwirtschaftssteuern über einen Zeitraum von 5 Jahren gesenkt und die staatlichen Aufkaufpreise um 400-600\% angehoben. Die monopolistische Stellung der Kooperativen blieb bei der Güterverteilung unverändert. In der zweiten Reformphase im April 1988 wurde die landwirtschaftliche Nutzfläche quasi dekollektiviert. Das Land wurde über langfristige Nutzungsrechte an private Personen zurückgegeben. Die Preisliberalisierung sowie der Wegfall der Subventionierung landwirtschaftlicher Produktionsmittel ermöglichte den Familien nun nach marktwirtschaftlichen Prinzipien zu wirtschaften (KURTHS, 1997).

\subsubsection{Das Bodengesetz}

Seit das Bodengesetz 1993 überarbeitet wurde, ist es Privatpersonen möglich, Nutzungsrechte für verschiedene Landkategorien zu erwerben. Privater Landbesitz ist untersagt, die Fläche Vietnams befindet sich nach wie vor in Staatseigentum. Die Landnutzungsrechte werden von einem Volkskomitee vergeben. Sie werden für einen Zeitraum von 15- 20 Jahren für landwirtschaftliche Nutzflächen und von 30-50 Jahren für forstwirtschaftlich genutzte Flächen vergeben (LAND LAW OF VIETNAM, 1993). Die Größe der Privatpersonen zugewiesenen Fläche richtet sich nach den Fähigkeiten und Möglichkeiten des Betriebsleiters, der die Fläche bewirtschaftet (UNDP\& FAO 1996). Es besteht die Möglichkeit, diese Landnutzungsrechte zu verkaufen, zu verpachten und zu vererben. Ein Bodenmarkt ist nicht existent. Es besteht allenfalls ein Markt für Landnutzungsrechte (KURTHS, 1997).

\subsubsection{Umweltschutzprogramme}

Vor 1992 wurde die Waldfläche Vietnams durch Abholzung jährlich um ca. 110.000 ha reduziert. Als Folge sind verstärkte Bodenerosion, Verminderung der biologischen Vielfalt, Brennholzknappheit und sozialer Unfriede zu beobachten. 
Tab.4: Die Veränderungen der Waldfläche in Vietnam von 1943 bis 1993

\begin{tabular}{lrr}
\hline Jahr & Gesamtwaldfläche (1000 ha) & \% der Landesfläche \\
\hline 1943 & 13.500 & 40.7 \\
\hline 1975 & 9.500 & 28.6 \\
\hline 1983 & 7.812 & 23.6 \\
\hline 1993 & 9.100 & 27.7 \\
\hline
\end{tabular}

Quellen: (Do, D. S, 1994)

Um diesen negativen Auswirkungen $\mathrm{zu}$ begegnen, wurden verschiedene staatliche Umweltschutz- und Wiederaufforstungsprogramme durchgeführt. Das 1992 verabschiedete Programm 327 steht dabei an erster Stelle. Es räumt der Entwicklung von degradierten Flächen oberste Priorität ein. Die Verbindung von Pflanzen- und Tierproduktion und Forstwirtschaft, gekoppelt mit verstärktem Schutz von Waldflächen und Wassereinzugsgebieten, bildet wichtige Pfeiler des Programmrahmens. Vorrangige Ziele des Programms sind unter anderem die Wiederbegrünung von degradierten Berghängen, der Schutz von Waldflächen und der Biodiversität (FAO, 1996). 

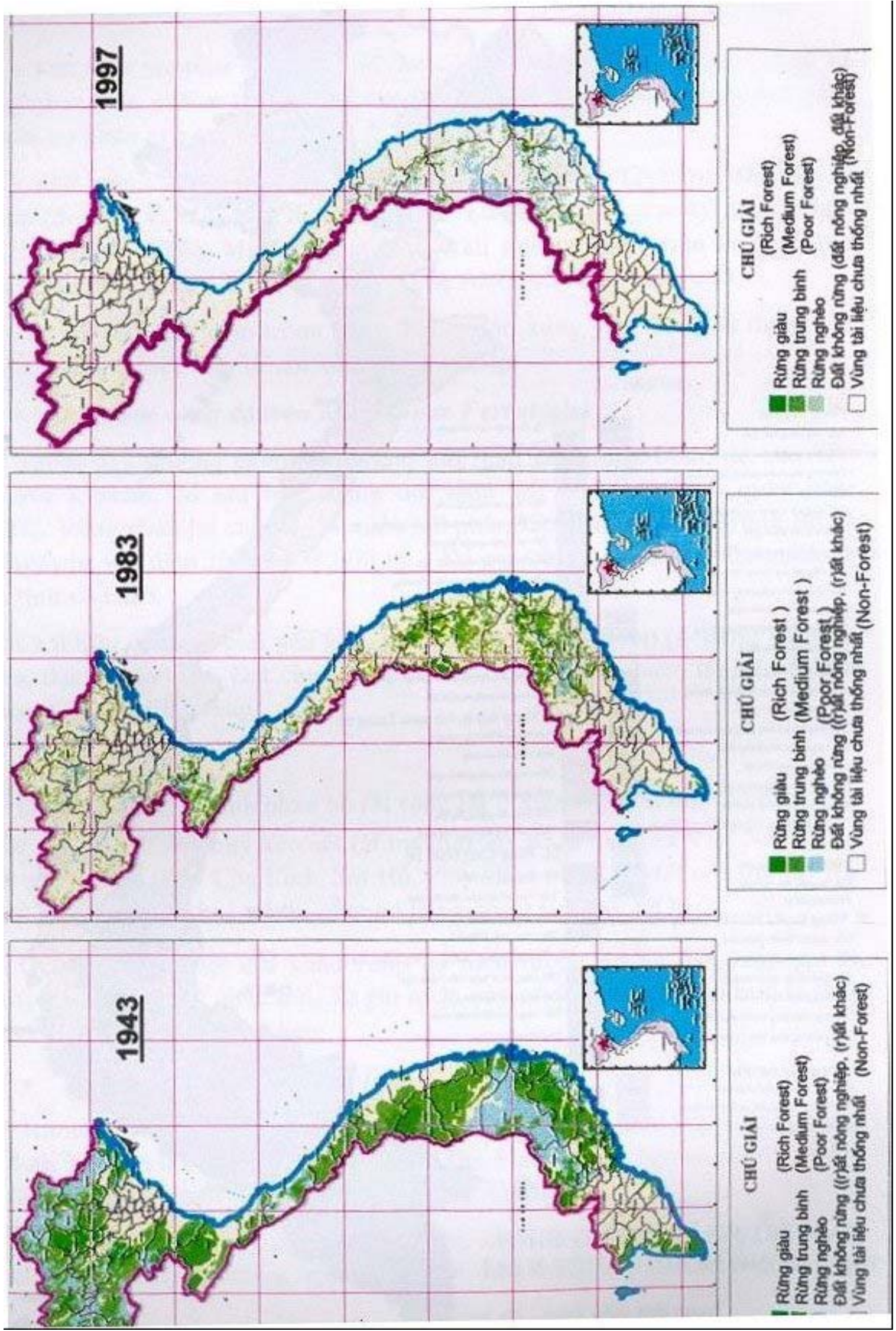

Karte 2: Die Veränderungen der Waldfläche in Vietnam nach 54 Jahre ( Quellen: Do, D. S, 1994) 


\subsection{Die Untersuchungsregionen}

Die der Arbeit zugrundeliegenden regionalen Daten sind von den statistischen Abteilungen des Distrikts Ba Be und der Provinz Thai Nguyen zur Verfügung gestellt worden.

\subsubsection{Geographische Lage}

Die Fläche der Provinz Bac Can beträgt 479.554 ha, davon weisen 25.383 ha (5,2\%) eine Hangneigung von $0-7^{\circ}, 56.177$ ha $(11,7 \%)$ von $8-15^{\circ}$ und 397.994 ha $(83,1 \%)$ von mehr als $16^{\circ}$ auf.

Das Untersuchungsgebiet Dia Linh hat eine Fläche von 3.311 ha und befindet sich im 1.151.730 ha großen Distrikt Ba Be der Provinz Bac Can. Der Distrikt Ba Be liegt sich zwischen $105^{\circ} 40^{\prime}-105^{\circ} 47^{\prime}$ östlicher Länge und $21^{\circ} 20^{\prime}-22^{\circ} 23^{\prime}$ nördlicher Breite. An ihn grenzen im Norden die Distrikthauptstadt Cho Ra, im Osten der Distrikt Banh Trach, im Süden der Distrikt Yen Duong und im Westen der Distrikt Thuong Giao an. 94,3\% der Fläche von Babe sind Berge und Hügel mit Höhen zwischen 200 und 1400 m über NN (Karte 1). Ihre Hangneigung reicht bis zu $40^{\circ}$. Eine flächenmäßige Ausdehnung der landwirtschaftlichen Produktion in diesem Gebiet ist hinsichtlicht der Oberflächengestalt mit großen Problemen verbunden.

Das Untersuchungsgebiet ist $90 \mathrm{~km}$ von der Provinzstadt Bac Can bzw. $254 \mathrm{~km}$ von der Hauptstadt Hanoi entfernt und ist in sechs Autostunden von Hanoi aus zu erreichen. Seine Entfernung zu dem im Jahr 1977 gegründeten und weitverbreitet bekannten Nationalpark Ba Be beträgt $10 \mathrm{~km}$. 


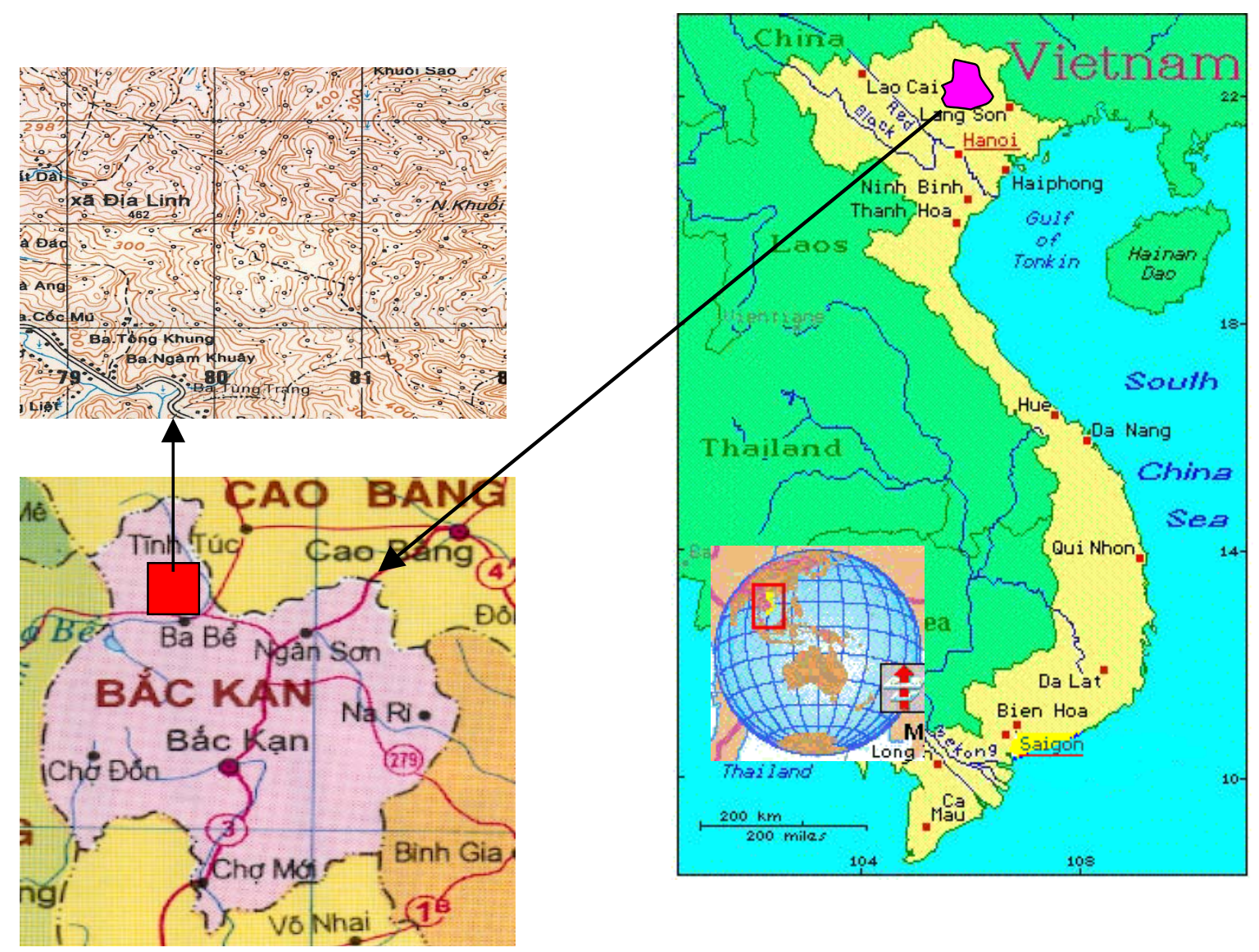

Abb. 1: Arbeitsgebiet

\subsubsection{Geologie}

Die geologische Karte in Abb. wurde auf Grundlage der geologischen Karte von Bac Kan 1: 200000 erstellt.

Daraus ist ersichtlich, dass im Untersuchungsgebiet überwiegend paläozoische Sedimentgesteine des Ordovizium und Silur anstehen. Sie untergliedern sich in 2 Unterformationen mit ähnlichem Aufbau: mächtige Wechselfolgen von Tonstein/schiefer, Schluffstein, Sandstein, die teilweise kalkhaltig sind. In der mittleren Unterformation des Ordovizium-Silur sind nach der geologischen Karte auch Kalkgesteine in die Sedimentfolgen eingeschaltet. Das Vorkommen von Tonschiefer gibt Hinweis auf eine schwache metamorphe Überprägung der Sedimentgesteine. Die unterschiedlich restistenten paläozoischen Sedimentgesteine werden im Süden von granitischen und granidioritischen Intrusivgesteinen des Trias abgelöst. Sie bilden die höchsten Gipfel über $900 \mathrm{~m}$ ü NN.

Die Untersuchungsparzellen befanden sich im Bereich der mittleren Unterformation des Ordovizium - Silur. 


\section{Karte 3: Geologisch - geomorphologische Verhältnisse im Dia linh, Babe Disktrick}

,<smiles>[R][SbH]=[SeH]</smiles>

H.: 2484700
R: 581505

H.: 2484700

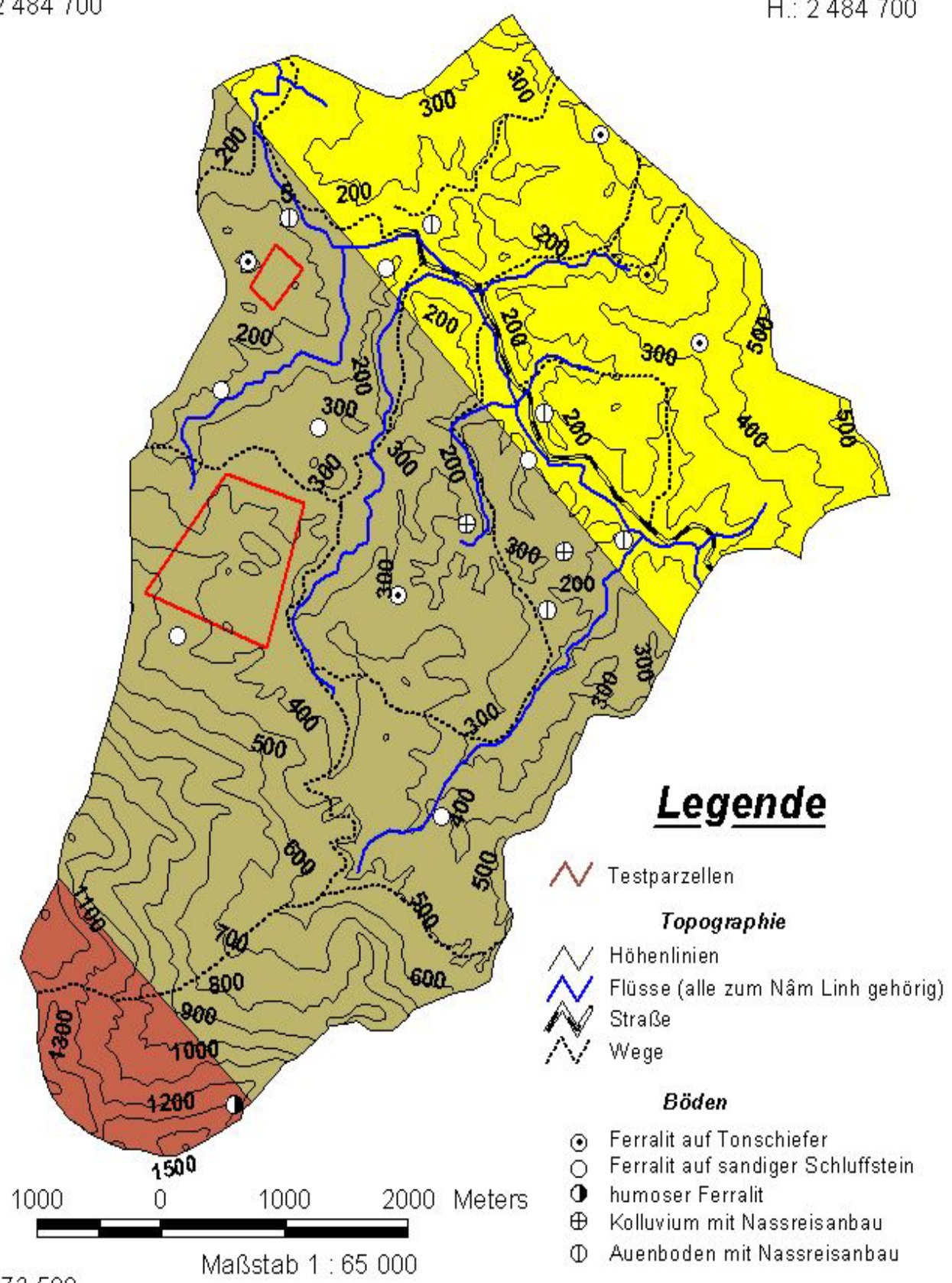

R.: 573500

H.: 2475000

\begin{tabular}{|l|}
\hline Kartengrundlagen \\
TK 50 Cho Ra (F-48-56-C) \\
TK $50 \mathrm{Na}$ P hac (F-48-56-D) \\
Geologische Karte Bac Kan \\
1:200 000 (F-48-XVI) \\
Administrative Karte $\mathrm{B}$ ac Kan \\
1:500 000, aus "Viet Nam \\
Administrative Atlas", \\
Cartographic Publishing House, \\
Ha Noi, 2002 \\
Bodenk arte $\mathrm{Ba}$ Be (unveröftentlicht)
\end{tabular}

\section{Geologie}

Ordovizium-Silur; Tonschiefer, sandiger Schluffstein, Sandstein eingelagert mit dünnen Schichten von kieseligem Tonstein, kalkhaltiger sandiger Schluffstein, $1125 \mathrm{~m}$ mächtig Ordovizium-Silur, schwarzer Tonschiefer, kieseliger Schiefer, tuffig-sandiger Schluffstein, Kalkstein-Linsen, toniger Kalkstein; 1300 m mächtig Trias; Granodiorit, mittel- bis grobkörniger porphyrischer Biotit Granit 


\subsubsection{Klima}

Im Untersuchungsgebiet herrscht ein randtropisch wechselfeuchtes, monsunales Klima. Die durchschnittliche Luftfeuchtigkeit liegt bei 82\%. Die Sonne scheint etwa 1.347 h/Jahr. Die Jahresdurchschnittstemperatur beträgt $22,8^{\circ} \mathrm{C}$. Der kälteste Monat Januar hat eine durchschnittliche Temperatur von $14,2^{\circ} \mathrm{C}$. In den folgenden Monaten steigt die Temperatur allmählich und erreicht ihren maximalen Werte mit $28,5^{\circ} \mathrm{C}$ im Juli. Die monatliche Niederschlagsmenge steigt rasch von Februar bis Juli und erreicht ihren Höhepunkt von $250 \mathrm{~mm}$ im Juli und August. (s.Abb. 2). Die mittlere jährliche Niederschlagsmenge beträgt 1480 mm im langjährigen Mittel von 1996-2000. 85\%- 90\% des Niederschlages fallen von Mai bis September.
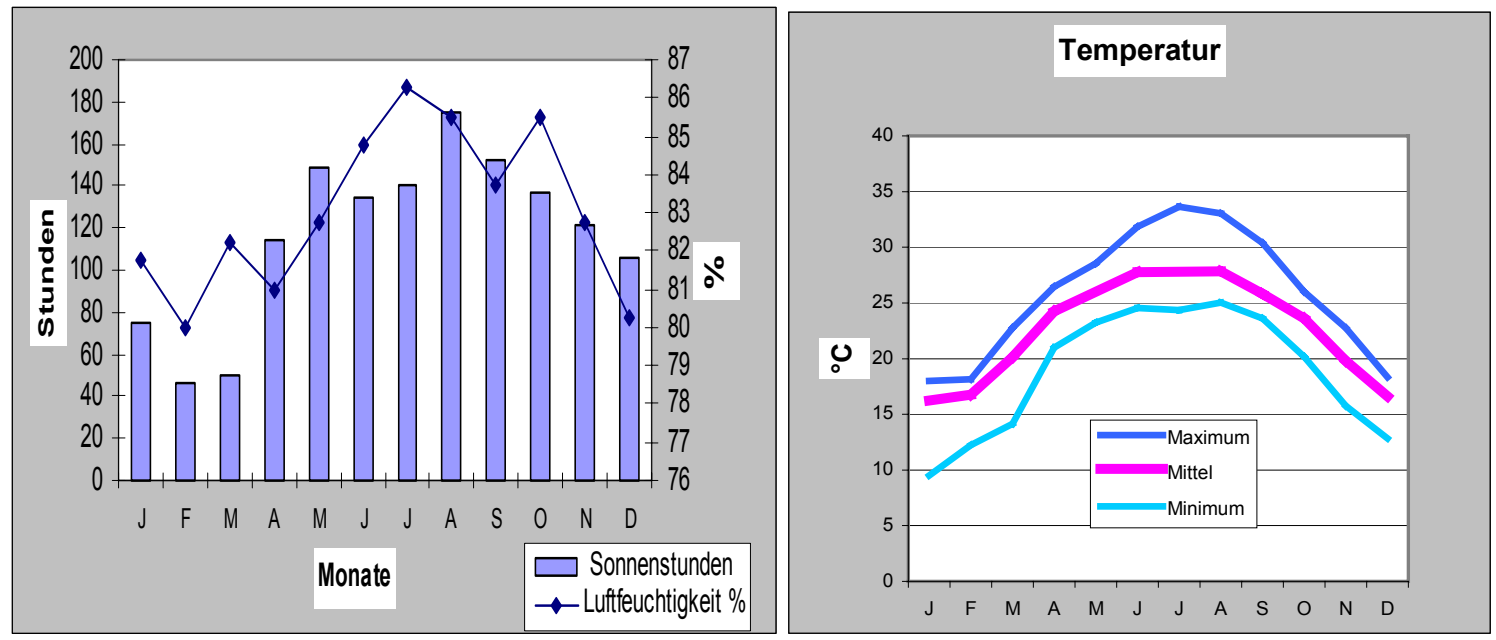

Abb. 2: Durchschnittliche Klimadaten des Distrikts Ba Be in den Jahren 1996-2000 Quelle : Daten der Wetterstationen des Distrikts Babe

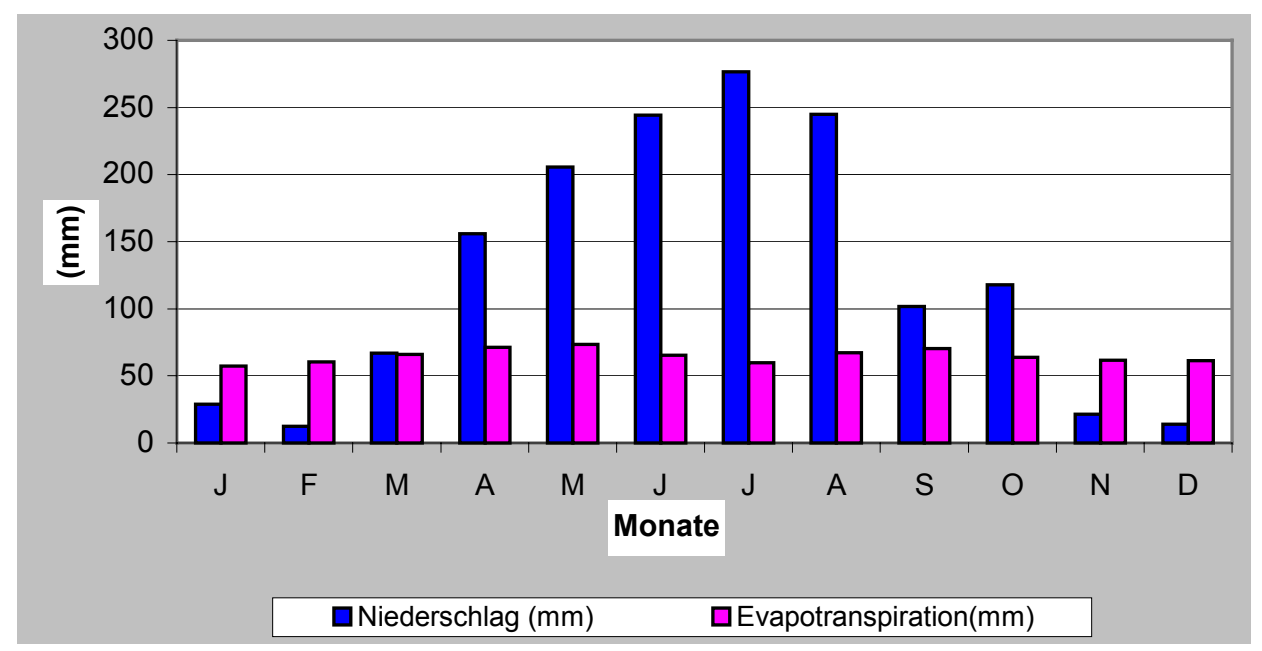

Abb. 3. Durchschnittliche Niederschlag und Evapotranspiration des Distrikts Ba Be in den Jahren 1996-2000 


\subsubsection{Demographische Merkmale}

Im Distrikt Babe leben 68.623 Menschen. Die Bevölkerungsdichte beträgt rund 59 Personen $/ \mathrm{km}^{2}$. Im Untersuchungsgebiet Dia Linh leben jedoch 3.314 Personen. Bei einer Gesamtfläche von 3.311 ha ergibt sich eine Bevölkerungsdichte von 100 Personen $/ \mathrm{km}^{2}$ (Stat. Jahrbuch des Babedistrikt 2001).

Im Vergleich dazu betrug die Bevölkerungsdichte 1975 nur 34 Personen/ km². Im Jahre 1979 stieg die Bevölkerung als Folge eines Krieges mit China plötzlich stark an. 200 Familien verließen die südlichen Distrikte der Caobang Provinz, die an China fielen, und siedelten nach Babe und Nahang um. In jüngster Zeit hat das Bevölkerungswachstum von 2,9\% im Jahre 1991 auf 1,9\% im Jahre 2000 abgenommen.

Es gibt vier ethnische Minderheiten: Tay, Dao, Nung und Kinh. Die überwiegende Mehrheit der dort ansässigen Bevölkerung gehört zu den Tay. Sie hat einen Anteil von $70 \%$, danach kommen die Dao mit 10\%.

\subsubsection{Landnutzung}

Die vorliegende Forschungsarbeit konzentriert sich hauptsächlich auf einen landwirtschaftlich intensiv genutzten Raum. Der hohe Anteil der Ackerflächen an der Gesamtfläche des Gebietes hat sich im Laufe der Zeit stark verändert. Das Untersuchungsgebiet Dia Linh im Distrikt Ba Be ist eine für das Projektgebiet typische Gebirgsregion: schmale Tälern und hoch aufragenden Bergketten mit steilen Hängen. Mehr als 80\% der Distriktfläche sind als Wald klassifiziert.

Das aktuelle Landnutzungssystem vom Dia Linh stellt sehr komplex dar und umfasste neben dem heute vorrangig praktizierten Brandrodungsfeldbau den Fischfang, das Sammeln von Waldfrüchten, Honig und Medizinalpflanzen und die Jagd. Durch die Kleintierhaltung (Hühner, Enten, Schweine) tritt das eigentlich Motiv des Jagens - die Versorgung mit Proteinen- mehr und mehr in den Hintergrund. Die Waldnutzung belief sich den bereits erwähnten Aktivitäten auf das Sammeln von Brennholz und das Schlagen von Bauholz. Die Waldbedeckung beträgt zur Zeit aber nur noch etwa 22\%. Starkes Bevölkerungswachstum, Umsiedlungen von Menschen aus den dichtbesiedelten Gebieten der Provinz Bac Can und unkontrollierte Holznutzung verstärken den Druck auf die verbliebenen Waldressourcen. Sowohl Tay als auch Nung und Kinh müssen ihre landwirtschaftlichen Flächen in immer steilere Berghänge ausweiten, Bracheperioden 
werden verkürzt. Dies führt zur Degradierung oder zum Verlust von landwirtschaftlichen Nutzflächen und schließlich zur Minderung der Lebensqualität für die Bevölkerung.

Verschiedene Projekte versuchen, zur Stabilisierung der Agrar- und Forstökosysteme sowie zur nachhaltigen Verbesserung der Lebensgrundlagen der lokalen Bevölkerung beizutragen.

\section{Landwirtschaft}

Der landwirtschaftliche Sektor nimmt im Distrikt $\mathrm{Ba} \mathrm{Be}$ eine dominierende Rolle ein. 92\% des Bruttosozialproduktes des Distriktes werden von diesem Sektor erwirtschaftet. Die Erwirtschaftung der restlichen 8\% des Bruttosozialproduktes teilen sich auf die Sektoren Industrie und Handwerk sowie Dienstleistungen auf.

Die nachstehende Skizze soll ein ökologisches System der Landwirtschaft des Untersuchungsgebiets dokumentieren. In Abb.4 ist ein Talquerschnitt durch ein traditionelles Landwirtschaftsökosystem des Untersuchungsgebietes dargestellt.

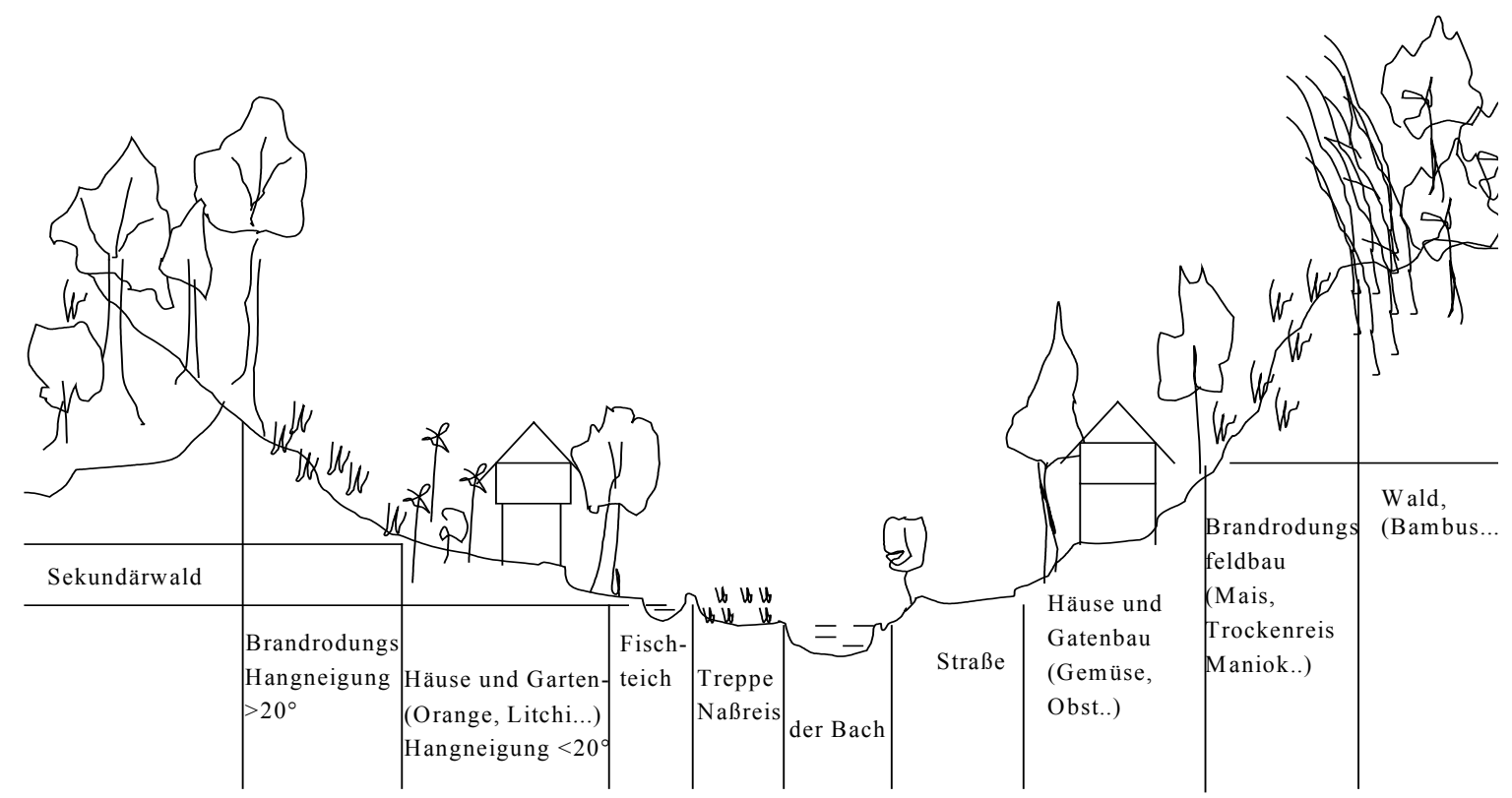

\section{Abb. 4: Tal-Querschnitt eines Landwirtschaftsökologiesystems vom Dorf Dia Linh,} Ba Be

Quelle: eigene Erhebung

Einem typischen Haushalt stehen etwa 0,5 ha Nassreisanbauflächen, 0,1 ha Hausgärten, 0,1 ha Brennholzforsten, 0,1 ha Teeplantagen, 0,5 ha sonstige Anbauflächen (Mais, Erdnüsse o.ä.), 0,2 ha Medizinalpflanzen-Anbauflächen im Wald,1- 3 Wasserbüffel, 2 Schweine und 10 Hühner zur Verfügung. Wasserbüffel dienen als Zugtiere im Reisanbau, aber auch der Fleischerzeugung. Hühner-, Schweine-, Rinder- und teilweise Entenzucht sind weitverbreitet. Darüber hinaus kann jeder Haushalt forstliche Produkte auf der 
gesamten Dorfwaldfläche nutzen. Die Feldarbeit wird von allen arbeitsfähigen Mitgliedern eines Haushaltes gemeinsam verrichtet. Waldarbeit, Jagd, Fischfang und Hausbau ist Sache der Männer, das Sammeln, Weben und Kochen übernehmen die Frauen. Alle Arbeiten werden mit einfachen Gerätschaften durchgeführt.

\section{Traditionell lassen sich 3 Landnutzungssysteme unterscheiden:}

\section{Naßreis}

Nassreisanbau wird auf überfluteten Feldern im Auenbereich der Flüsse und Bäche bzw. als Terrassenanbau im unteren Bereich der Talhänge betrieben. Die Felder sind sehr klein. Terassennaßreisanbaut auf Hang werden überwiegend nur 1x pro Jahr während der Regenzeit bestellt. Die Tay bauen ausschließlich Naßreis an. Obwohl ein zwei- bis dreimaliger Anbau in Babe möglich ist, wird in Dialinh, wie auch in den meisten NaducDörfern, Reis nur einmal jährlich angebaut. Gründe dafür sind die hohe Arbeitsintensität bei geringem Maschineneinsatz und die Tatsache, dass die Bewässerung zur Verfügung nicht genug im Trockenzeit ist. Die Anbauperiode erstreckt sich von Ende Mai bis Anfang Oktober und erfolgt damit in der Regenzeit. Die Terrassierung der Felder ermöglicht eine technisch einfache, flußgespeiste Bewässerung.

In der Trockenzeit werden flussnahe Felder weiter bewässert und Gemüse, häufig Sauropus androogynus (rau ngot), Rau muong, angebaut. Seit der Landreform 1992 ist die landwirtschaftliche Fläche auf die Haushalte aufgeteilt. Jeder Haushalt bewirtschaftet ca. 0,7 - 0, 8 ha Reisfelder.

Die durchschnittlichen jährlichen Reis-Erträge in Dialinh liegen bei $3,1 \mathrm{t} / \mathrm{ha}$. Spitzenerträge bei zweimaliger Ernte liegen bei 5,6 t/ha. Traditionelle Reissorten, z. B. der glutinose („klebrige“) Reis, bringen nur einen Ertrag von 800 kg/ha, werden aber aufgrund ihrer Beliebtheit mit angebaut. Die Marktpreise für die traditionellen Sorten sind drei- bis viermal so hoch wie für die Hochertragssorten. Bei allen traditionellen Festen der Tay wird ausschließlich der glutinose Reis gegessen.

\section{Hausgärten}

Tropische Hausgärten mit einer Mischung verschiedenster Obst- und Gemüsearten im Bereich der Siedlungen stehen ganzjährig zur Ernährung seiner Bewohner zur Verfügung. Hausgärten sind ein typisches Element in traditionellen Landnutzungssystemen der ethnischen Minderheiten in Dialinh. Als ein Element einer nachhaltigen Landnutzung ist dieses agroforstliche System für die Tay- Minderheit charakteristisch. In Dia Linh hat 
jeder Haushalt einen 20-2000 $\mathrm{m}^{2}$ großen Hausgarten, wobei die Größe von der Dorfmitte zur Peripherie hin zunimmt. Neben Bambus und Fruchtbäumen werden Gemüse, Gewürze, Medizinal- und Futterpflanzen sowie ornamentale Pflanzen angebaut. Darunter finden sich viele Arten, die früher im Wald gesammelt wurden. Im randtropischen Bereich befindlich, zeigen die Hausgärten Anpassungen an die Periode der Trockenzeit, die sich bis zu 5 Monaten ausdehnen kann. Auch die Besonderheiten, die sich durch die Wasserbüffelzucht der Tay ergeben, sind erwähnenswert. Jede der vielen kleinen Parzellen der Hausgärten ist umzäunt von stachligen und trockenresistenten Hecken oder Bambuszäunen. Nur Hühner sind ein integraler Bestandteil der Hausgärten. Vorherrschend sind einschichtige Gärten. In der Trockenzeit befinden sich keine annuellen Pflanzen in ihnen, außer in denen, die von den Veranden der Pfahlhäuser herab bewässert werden. Im Frühjahr wird von jungen Blättern und Trieben der Bäume Gemüse gewonnen. Düngung spielt nur eine untergeordnete Rolle. Tierdung aus den in den Gärten befindlichen Schweineställen wird nur in geringem Maße verwendet. Durch freilaufendes Geflügel wird Kot ungezielt in die Systeme eingebracht. Die Hausgärten in Dialinh dienen zu etwa 90\% dem täglichen Bedarf der Familie, es werden nur wenige Cash Crops angebaut.

\section{Bergfeld}

In höhergelegenen Bereichen wird von den Bauern der Anbau als Wanderfeldbau mit Brandrodung betrieben. Diese Felder sind zum Teil mehrere Kilometer von den Siedlungen entfernt. Der Anbau erfolgt in Monokultur mit 1 Ernte pro Jahr am Ende der Regenzeit. Hauptkulturen sind Mais, Maniok und Trockenreis. Nach dem Anbau über 35 Jahre folgt heute eine etwa gleichlange Brachezeit. Aufgrund der Kürze der Brachezeit kann sich nur eine Buschbrache etablieren bevor das Feld erneut genutzt wird. Die Brachezeit ist außerdem zu kurz, um eine Regeneration der Böden zu ermöglichen. Nach etwa 3 Nutzungszyklen (18 - 30 Jahre), wenn sich Grasbrache mit Alang Alang einstellt, sind die Flächen unnutzbar und werden aufgeben. Zum Ausgleich werden neue Flächen durch Brandrodung von Primärwald oder Sekundärwald einer Nutzung zugeführt.

Die Bewirtschaftungsintensität ist auf Flächen mit höherer Hangneigung in den letzten 10 Jahren stark angestiegen. Flächen über $20^{\circ}$ Hangneigung werden intensiv für den Wanderfeldbau herangezogen

Die aktuelle landwirtschaftliche Nutzung im Babe entspricht bei weitem nicht den Vorstellungen der Planer. 


\section{Waldnutzung}

\section{Die Zerstörung der Waldressourcen im Babe Distrikt von 1954-1981}

1954: Bodenreform; die gleichmäßig verteilte Armut innerhalb der Bevölkerung in Babe bedingt eine einfache Bodenaufteilung.

1960: Im September werden mit Hilfe der Regierung Genossenschaften gegründet. Nach Auskunft eingeborener Bauern, war der Primärwald zu diesem Zeitpunkt noch unbeschädigt. Es gab noch viele Hirsche und Tiger und keine umformende Landnutzung. 1979: China übernimmt einige südliche Distrikte der Caobang Provinz. Es kommt zur Auswanderung von 200 Familien nach Babe und Nahang. In nur einem Jahr fallen dort 2000 ha Wald dem traditionellen Wanderfeldbau zum Opfer. Um die wachsende Bevölkerung zu ernähren, müssen die Bauern ihren Anbau auf steile Berghänge ausweiten.(PHAN. X. D, 2001)

1981: Die Genossenschaften lösen sich auf. Armut und Hunger werden nun zum schweren Problem. Die eingeborenen Bauern sprechen außerdem von einer Zerstörung der Waldressourcen in Babe im Zeitraum von 1970-1982 um etwa 40\%. Ursachen sehen sie zum einen in der, durch die Einwanderer ständig waschenden Bevölkerung sowie im schlechten Management der Genossenschaften.

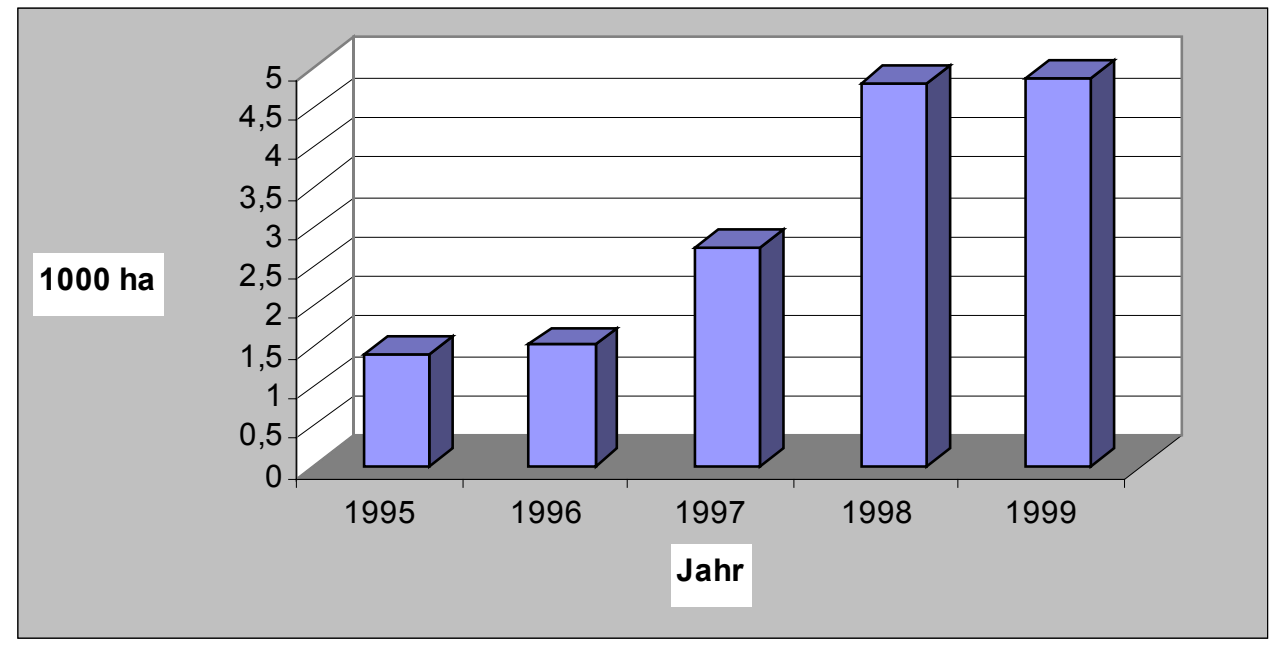

Abb. 5: Aufforstungen in der Provinz Bac Can 1995-1999

Quelle: Statistik Jahrbuch 1999 (Bac Kan Statistik Abteilung)

Seit 10 Jahren bemüht sich die Regierung Vietnams mit Hilfe von unterschiedlichen Programmen den Wald zu erhalten bzw. zu vermehren. Ihre wichtigsten Ziele bestehen darin, die Aufforstung auf kahlen Flächen zu fördern und die Hungersnot zu bekämpfen. Im Jahre 1993 wurde das Programm zur Hunger- und Armutsbekämpfung, der Familienplanung und Förderung der Sesshaftigkeit (genannt: Programm 327) 
verabschiedet. Mit diesem Programm wurde die staatliche Waldbodenfläche Privathaushalten zugeteilt. Die am Wald lebende Bevölkerung soll mit diesem Programm beim Anbau, der Erhaltung sowie der Verwaltung des Waldes mitbestimmen und eigene Verantwortung dafür übernehmen. Aufgrund der Umsetzung dieses staatlichen Programms hat die Waldfläche der Provinz Bac Can nach 1992 zugenommen. Die Anbaufläche im Jahre 1995 mit rd. 1.000 ha hat sich auf ca. 5.000 ha verfünffacht.

Bei der Durchführung des Programmes setzte man auf die Mitarbeit der ansässigen Bauern. Für ihre erbrachte Arbeitsleistung wurden sie pro Hektar aufgeforstete Fläche mit mit $50 \mathrm{~kg}$ Reis oder 40- 50 Euro/ ha entlohnt. Material und Technik wurden vollständig durch das Programm zur Verfügung gestellt.

\subsubsection{Böden}

In Abb. 6 ist der flächenmäßige Anteil von Bergfeldern, Sekundärbusch, Brache und Erosionsflächen im Ba Be - Distrikt für 2001 dargestellt. Sekundärbusch mit Grasbewuchs und einzelnen Sträuchern (meisten Alang Alang - Imperata cylindrica) bedeckt rund 36.218 ha. Das entspricht rund $2 / 3$ der gesamten forstwirtschaftlichen Fläche des $\mathrm{Ba} \mathrm{Be}$ - Distriktes (s. Abb. 7). Es handelt sich dabei um eine Degradationsstufe bzw. Ersatzgesellschaft des Primärwaldes in Folge von Übernutzung des Primärwaldes (u.a. durch Brennholznutzung, Waldweide) bzw. traditioneller Brandrodung. Die Böden sind sehr arm an Nährstoffen und daher nur noch für forstliche Nutzung geeignet. Ca. 550 ha sind nach vollständiger Degradation der Vegetation durch Bodenerosion jeglicher Nutzung entzogen.

Eine Herausforderung für die Wissenschaft liegt in der Frage: Wie kann man die Bodenqualität zu Gunsten der Landwirtschaft verbessern bzw. erhalten? 


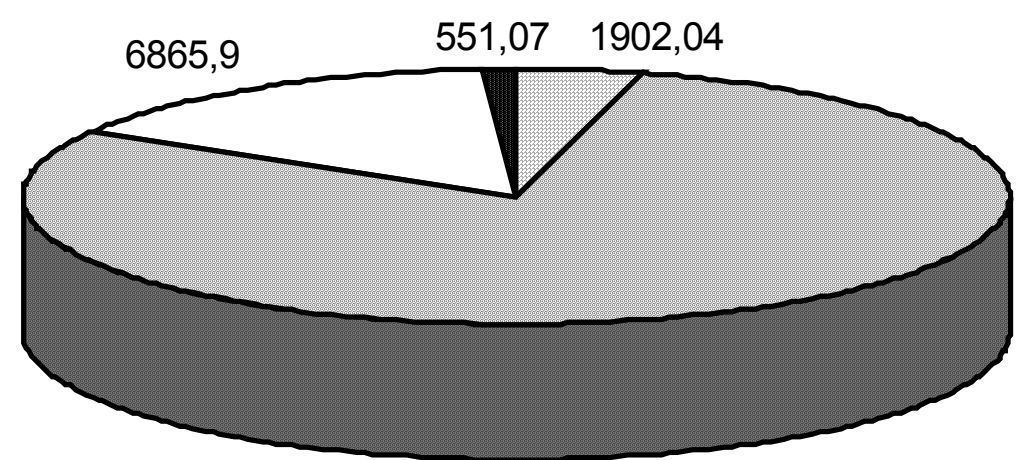

36218,6

\begin{tabular}{|lll|}
\hline Bergfeld $\quad$ QSekundärbusch $\quad$ Brache $\quad$ Erosionflächen \\
\hline
\end{tabular}

\section{Abb.6: Feldbau-, Sekundärbusch- und Brachefläche im Ba Be Distrikt 2001, Angaben in Hektar}

( Aus: Statistik Ba Be Distrikt, 2000)

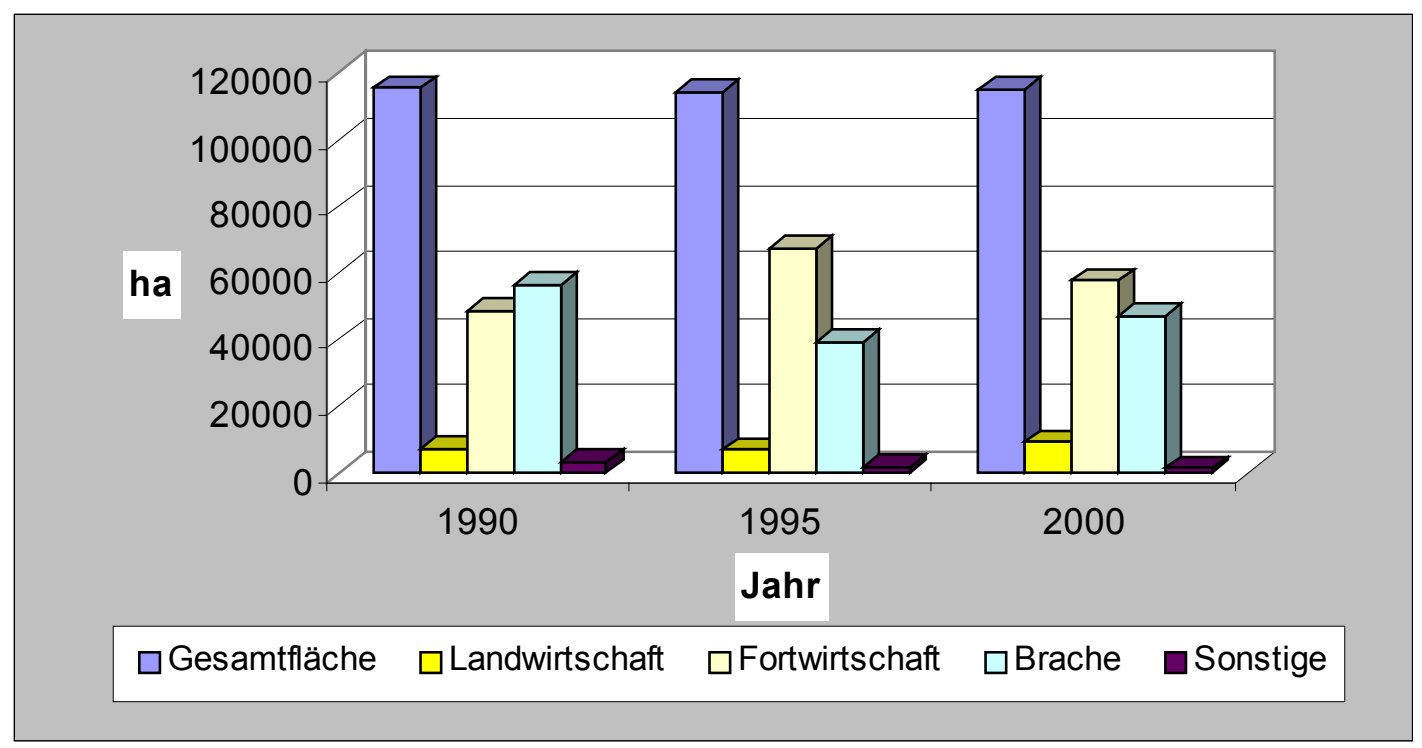

Abb.7: Graphische Darstellung der Flächennutzung des Distriktes Ba Be (Aus: Statistik Ba Be Distrikt 2000)

Aus der oben dargelegten graphischen Darstellung wird deutlich, dass die Gesamtfläche des Distrikts $\mathrm{Ba} \mathrm{Be}$ in den drei Erhebungsjahren 1990, 1995 und 2000 unverändert geblieben ist. Die forstlich genutzte Fläche stieg zunächst auf knapp 18.424 ha im Jahre 1995 und reduzierte sich danach auf 9.641 ha im Jahre 2000 zugunsten der landwirtschaftlichen Nutzung. Eine Zunahme der forstlichen Fläche in 1995 liegt in einem staatlicherseits durchgeführtem Aufforstungsprogramm.

Die Lösung der Bodenerosionsprobleme wird nicht allein durch technische Maßnahmen erreicht werden, sondern es gibt einen gleichermaßen wichtigen sozioökonomischen Zusammenhang 


\section{METHODIK DER UNTERSUCHUNG}

\subsection{Methodik der Fragenbogenerhebungen}

Für die Erhebung von Primärdaten wurden die beiden Dörfer Dialinh und Caotri mit 45 Bauernfamilien im Babe Distrikt der Provinz Baccan ausgewählt.

Die Durchführung der Datenerhebung fand im Zeitraum April und Mai 2001 statt. Um ein Bild von den lokalen Rahmenbedingungen $\mathrm{zu}$ erhalten, wurde vor Beginn der Einzelinterviews der Arbeitkalender und ein Transsekt erarbeitet. Die Erstellung des Transsekts erfolgte durch Dorfbegehungen in Gruppen, Gruppendiskussionen mit Vertretern der Gemeinde sowie eigene Beobachtungen. Der Arbeitkalender wurde mit der Datengrundlage der Einzelinterviews, klimatischen Daten der Wetterstationen BabeBaccan und Thai nguyen sowie Gruppendiskussionen erstellt.

Der Auswertung von Sekundärinformationen (Literatur, Projektberichte, statistische Daten verschiedener Administrationen, PRA- Ergebnisse) wurde besondere Wichtigkeit beigemessen, da über die Untersuchungsregion bereits umfangreiche Basisdaten verfügbar sind.

Die Statistikabteilung der Distriktverwaltung stellte alle verfügbaren sozioökonomischen, geographischen, klimatischen und landwirtschaftlichen Daten der Untersuchungsregionen zur Verfügung. Als Kriterien für die Auswahl dienten der Marktzugang, die ethnische Zugehörigkeit, die Diversität der Gehölzformationen und die vermutete unterschiedliche Nutzung der Gehölze.

Bei der Erhebung von Primärdaten kamen sowohl quantitative als auch qualitative Methoden zum Einsatz. Um die Eignung und den Umgang mit dem Fragebogen zu prüfen, wurde vor der Erhebung eine Voruntersuchung in Form von acht Interviews durchgeführt. Anschließend wurde der Fragebogen hinsichtlich aufgetretener Fehler überarbeitet und den Bedingungen der Untersuchungsgemeinden angepasst. Die Daten der Voruntersuchungs-Interviews wurden bei der Auswertung der Gesamtdaten nicht berücksichtigt.

Eine Gruppendiskussion im Beisein auswärtiger Personen kann erst nach größerer Vertrautheit mit den Dorfbewohnern erfolgen. Bei den Datenerhebungen innerhalb der Tay- Bevölkerungsgruppe gilt darum ein mehrmonatiger Aufenthalt zur Vorbereitung der Untersuchungen als Voraussetzung.

Das Vorgehen und die Methoden umfassten im Einzelnen:

- Kontaktaufnahme zu Dorfchefs und Bewohnern der Dörfer, 
- Individuelle Befragungen von Schlüsselpersonen,

- Quantitative Erhebungen mit standardisierten Fragebögen.

Die Dauer der Interviews sollte 90 bis 150 Min. nicht überschreiten, da sonst die Aufmerksamkeit der Befragten hätte nachlassen können, womit die Zuverlässigkeit der Angaben nicht mehr gegeben gewesen wäre. Im Anschluss an die Interviews wurden Feldbegehungen unternommen, um ein besseres Gesamtbild der Betriebe zu erhalten und die Erosion sowie die Erosionsschutzmaßnahmen zu beurteilen. Danach wurden die Fragebögen auf Vollständigkeit kontrolliert. Bei nicht zu klärenden Sachverhalten wurde der entsprechende Betrieb erneut aufgesucht, um Datenlücken zu schließen.

Bei Gruppendiskussionen sollte besonders auf die Partizipation der Frauen geachtet werden. Bei der Tay- Bevölkerung waren zunächst getrennte Gespräche mit Frauen und Männern durchzuführen, da die Frauen sehr zurückhaltend sind und ihre Meinung in Anwesenheit der Männer nicht frei äußern.

Ferneerfolgte eine Inventur der Landnutzung in den Dörfern zur Einschätzung der Waldzustände und Betriebssysteme.

Teilnehmende Beobachtung. Die Beteiligung an von Frauen ausgeübten Tätigkeiten und das Teilnehmen am täglichen Leben förderten das gegenseitige Vertrauen und die Offenheit zur Meinungsäußerung. Überdies ließen sich so tiefe Einblicke in die Lebensweise, Arbeitsabläufe und in das Management natürlicher Ressourcen gewinnen.

Es schloss sich dann die Datenanalyse und Bewertung der Ergebnisse mit einer Ausarbeitung von Vorschlägen für die Wald- und Agroforstwirtschaft an.

\subsection{Feldmethodik}

\subsubsection{Auswahl der Untersuchungsflächen}

Um die pedoökologischen Folgen des traditionellen Wanderfeldbaus im Vergleich mit modernen Landnutzungssystemen untersuchen zu können, mussten Chronosequenzen von sowohl traditionell als auch modern bewirtschafteten Parzellen gefunden werden, die folgenden Kriterien entsprachen:

Die Chronosequenzen sollten die Abfolge von der Brandrodung bis hin zu mehrjährig genutzten Flächen umfassen.

Die Flächen sollten möglichst dicht beieinander liegen und die gleiche Hangneigung, topographische Lage, bodenkundliche Einheit und klimatische Rahmenbedingungen aufweisen. 
Unter Berücksichtigung der vorgenannten Kriterien war es das Ziel, Untersuchungen für unterschiedliche Landnutzungen durchzuführen und dabei auch die soziale Stellung der Familienbetriebe zu berücksichtigen.

Es wurden 6 Parzellen mit unterschiedlicher Landnutzung ausgewählt:

- Sekundärwald,

- 1 Jahr nach der Brandrodung,

- 4 Jahre nach der Brandrodung (Ende der Fruchtfolge),

- 4 Jahre Brache,

- Alang Alang- Bewuchs,

- dreijährige agro- forstwirtschaftliche Nutzung (mit Mulch).

Der Bewuchs mit Alang Alang ist ein Indikator dafür, dass die Qualität des Bodens nicht mehr ausreichend ist, um Kulturpflanzen anzubauen.

Die Untersuchung und Beprobung der Untersuchungsflächen umfasste folgende Arbeitsschritte:

- Lagebestimmung der Beprobungsstandorte und der Profilgruben mit GPS und Fotodokumentation,

- Anlegen von Profilgruben bis zu einer Tiefe von $150 \mathrm{~cm}$ zur detaillierten Bodenansprache und Charakterisierung der Bodentypen (AG Boden 1994), Beprobung der Parzellen,

- Befragung der landbestellenden Bauern zur Bestimmung der nutzungsrelevanten Parameter.

\subsubsection{Chronosequenz: Beprobung der Untersuchungsflächen}

Nach Scheffer \& Schachtschabel (1992) befinden sich in den obersten $30 \mathrm{~cm}$ der Böden 90\% der Wurzelmasse der Vegetation. Da bei der Bearbeitung der Profilgruben bereits erkannt wurde, dass auch die tiefer liegenden Schichten eines Profils durchwurzelt sind, wurde die Durchwurzelung der Böden jedoch auch in einer Tiefe von 31 bis $60 \mathrm{~cm}$ gemessen. Die Entnahmetiefen für Ober- und Unterboden wurden demnach wie folgt festgelegt:

Oberboden:0-30 cm unter der Bodenoberfläche, Unterboden: 31-60 cm unter der Bodenoberfläche. 
Je Parzelle wurde jeweils eine Beprobung am Oberhang, Mittelhang und Unterhang vorgenommen. Jede Beprobung wurde als Mischprobe aus zehn Bohrstock- Einschlägen vorgenommen. Die Beprobungen erfolgten jeweils vor der Bestellung und vor der Ernte.

Tab.5: Schema der Bodenbeprobung

\begin{tabular}{|c|c|c|c|c|}
\hline \multirow{2}{*}{ Oberhang } & $\begin{array}{l}0-30 \quad \mathrm{~cm} \\
\text { (60 Einschläge) }\end{array}$ & $\begin{array}{l}3 \text { Untersuchungs- } \\
\text { punkte }\end{array}$ & $\begin{array}{l}\text { Je } 10 \text { Bohrstock- } \\
\text { Einschläge }\end{array}$ & \multirow{6}{*}{ Parzelle } \\
\hline & $\begin{array}{l}30-60 \quad \mathrm{~cm} \\
(60 \text { Einschläge })\end{array}$ & $\begin{array}{l}3 \text { Untersuchungs- } \\
\text { punkte }\end{array}$ & $\begin{array}{l}10 \text { Bohrstock- } \\
\text { Einschläge }\end{array}$ & \\
\hline \multirow{2}{*}{ Mittelhang } & $\begin{array}{l}0 \quad-\quad 30 \quad \mathrm{~cm} \\
(60 \text { Einschläge })\end{array}$ & $\begin{array}{l}3 \text { Untersuchungs- } \\
\text { punkte }\end{array}$ & $\begin{array}{l}10 \text { Bohrstock- } \\
\text { Einschläge }\end{array}$ & \\
\hline & $\begin{array}{l}30-60 \quad \mathrm{~cm} \\
(60 \text { Einschläge })\end{array}$ & $\begin{array}{l}3 \text { Untersuchungs- } \\
\text { punkte }\end{array}$ & $\begin{array}{l}10 \text { Bohrstock- } \\
\text { Einschläge }\end{array}$ & \\
\hline \multirow{2}{*}{ Unterhang } & $\begin{array}{l}0 \quad-\quad 30 \quad \mathrm{~cm} \\
\text { (60 } \\
\text { (6inschläge) }\end{array}$ & $\begin{array}{l}3 \text { Untersuchungs- } \\
\text { punkte }\end{array}$ & $\begin{array}{l}10 \quad \text { Bohrstock- } \\
\text { Einschläge }\end{array}$ & \\
\hline & $\begin{array}{l}30-60 \quad \mathrm{~cm} \\
(60 \text { Einschläge })\end{array}$ & $\begin{array}{l}3 \text { Untersuchungs- } \\
\text { punkte }\end{array}$ & $\begin{array}{l}10 \text { Bohrstock- } \\
\text { Einschläge }\end{array}$ & \\
\hline
\end{tabular}

\subsubsection{Versuchstandort und Methodik}

Die Auswahl der Standorte erfolgte nach eingehender Begutachtung des Geländes und Voruntersuchungen, die in Punkt 4.2.1 dokumentiert sind. Hierbei wurden der Anteil der angebauten Kulturpflanzen und, mittels eines Hangneigungsmessers, die Hangneigung festgestellt.

Ausgesucht wurden dann jeweils Standorte mit einer für die Region repräsentativen Hangneigung von $22^{\circ}(40,6 \%)$, bei mindestens 10 m Hanglänge. Zusätzliche Kriterien waren das Wachstumsstadium der Kulturpflanzen (Mais, Maniok) sowie der Bedeckungsgrad des Feldes.

Auf vier verschiedenen Standorten (unmittelbar nach der Brandrodung; 4 Jahre nach der Brandrodung; 4 Jahre Brache; Agro- Forstsystem mit Mulch) wurden vier Erosionsmessanlagen eingerichtet. Aus Tab. 6 sowie Abb. 8 können detaillierte Angaben zu den Erosionsmessanlagen, ihrer Gestaltung und den Rahmenbedingungen entnommen werden. 
Tab. 6 : Standortangaben der vier Erosionsmessparzellen im Einzugsgebiet Dialinh, Babe Distrikt.

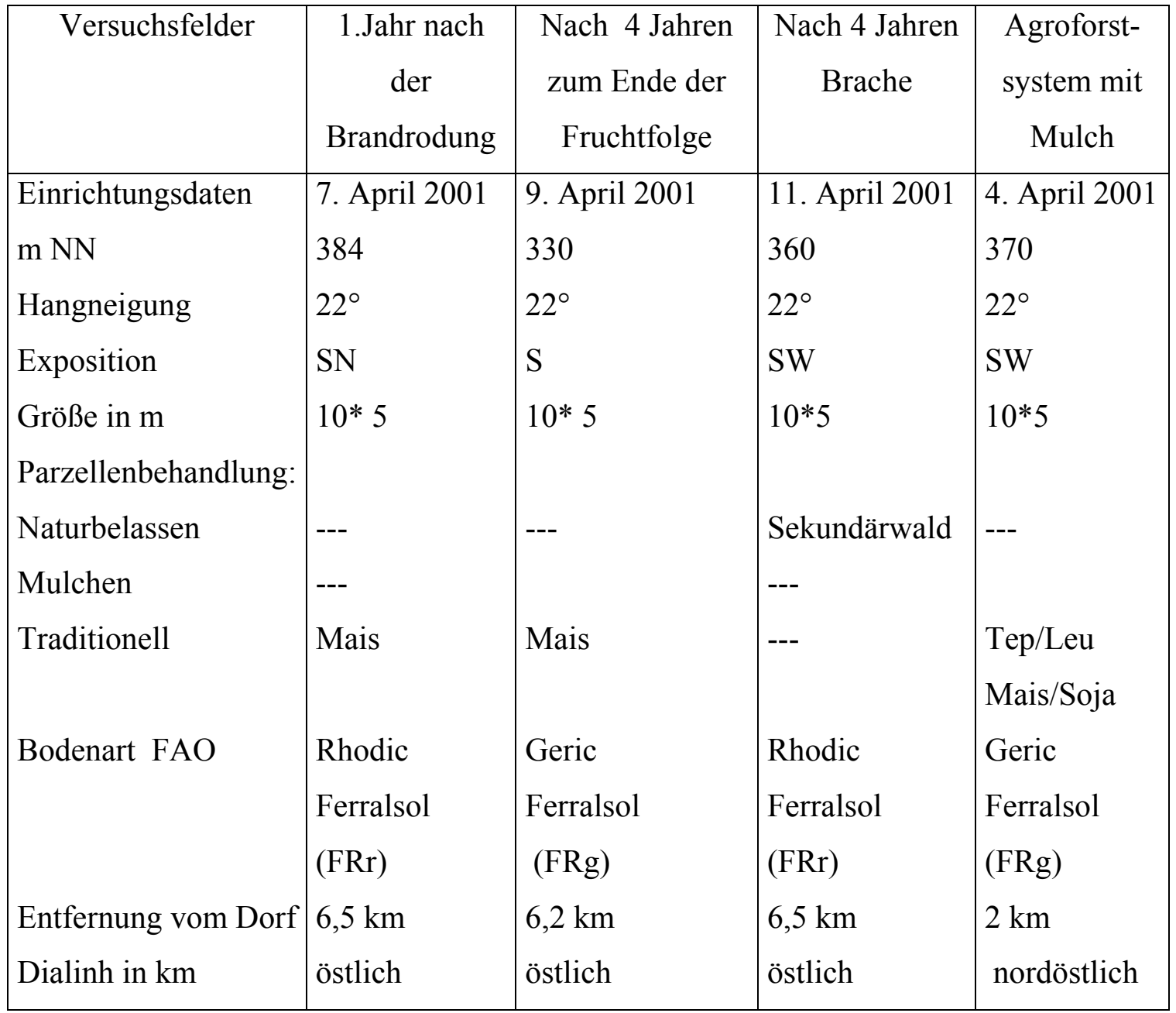

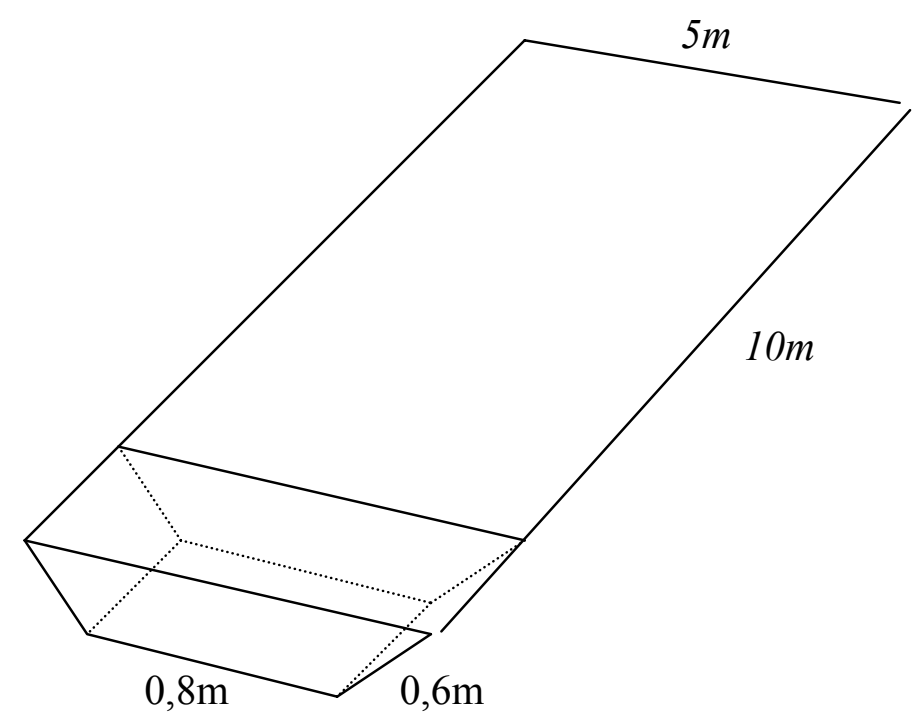

Abb. 8: Messparzellen 
Vier Parzellen wurden untersucht:

- 1.Jahr nach Brandrodung (Maisanbau)

- nach 4 Jahren zum Ende der Fruchtfolge (Maisanbau),

- nach 4 Jahren Brache,

- nach 3 Jahren mit Agro-Forstsystem (Hecken- Mulch- Maisanbausystem).

Das erste Versuchsfeld wurde einen Monat zuvor gerodet, nach vierjährigem Sekundärbuschbestand. Das zweite Versuchsfeld ist auf einer vier Jahre alten Parzelle mit Mais-Anbau eingerichtet worden. Das dritte Versuchsfeld lag, zur Erfassung der natürlichen Erosionsereignisse, in einem vom Menschen nicht gestörten, vier Jahre alten Sekundärwald, auf dem zuvor Maisanbau erfolgt war. Wegen dem Rückgang des Maisertrages überließ man diese Fläche der Brache. Das vierte Versuchsfeld befand sich auf einer dreijährigen Parzelle mit moderner Landnutzung (Agro-Forstsystem mit Maisanbau).

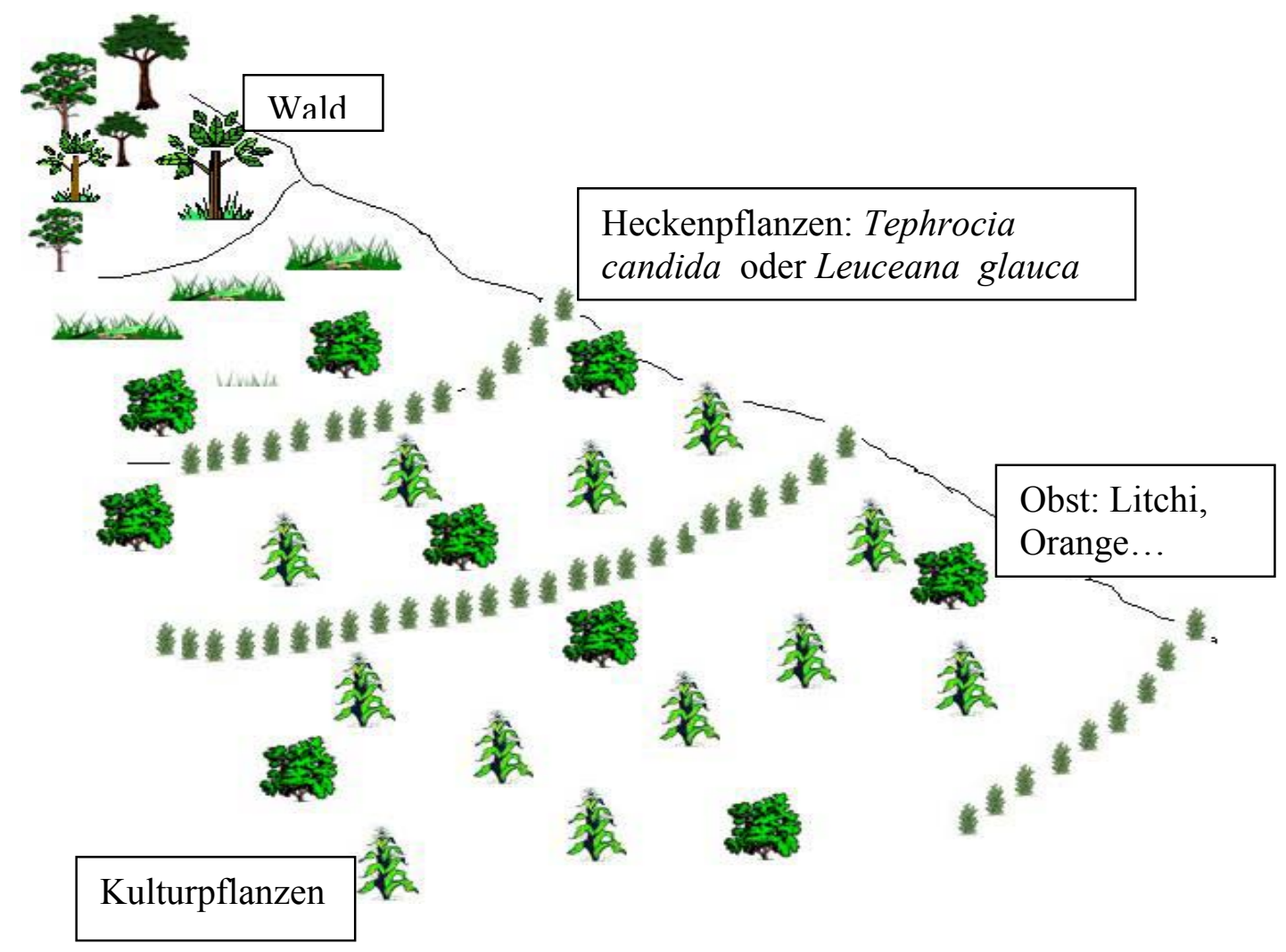

Abb.9: Schema Kleinbäuerliches Agro-Forstsystem mit Erosionsschutzmaßnahmen 
Für die neuen Nutzungssysteme wurden folgende Methoden in der Thainguyen Universität verwendet:

- Pflichtteil: Terrassierung mit Hecken zur Befestigung. Bei einem durchschnittlichen Pflanzabstand von 0,4 m innerhalb einer Reihe. Folgende Arten wurden dazu benutzt:

- Tephrosia candida (Cot khi), Vertiver

- Flemingia congesta, Leuceana glauca (keo dau)

Diese Pflanzen haben die Fähigkeit, sich schnell zu regenerieren. Dadurch können sie 3-4 mal im Jahr geschnitten werden.

- Wahlteil (optional): Reihenabstand von 5-6 m. Zwischen den beiden notwendigen Pflanzenreihen können andere Pflanzen angebaut werden, die je nach Klima und Nutzbarkeit zu wählen sind, z.B. Reis, Mais, Cassava, Obst, Grüntee etc. In dem Untersuchungsgebiet wurden Mais und Soja angebaut. Die Pflanzen sollten insbesondere als Bodenbedeckung in der Regenzeit dienen, um Erosion zu verhindern. Außerdem fand zwischen den Reihen Litchianbau mit einem Abstand von $5 \mathrm{~m}$ statt.

- Die Erosionsmessanlage zur quantitativen Erfassung des Bodenabtrages wurde nach dem Vorschlag von THAi PhIEN \& NGUYen TU SIEM 1999 eingerichtet. Die geographischen Daten des Geländes sowie der Messanlagen können aus Tab. 4 bzw. der Profilbeschreibung ( Punkt 6.1) und Abb. 8 entnommen werden.

Diese Testflächen hatten eine Größe von $50 \mathrm{~m}^{2}\left(5^{*} 10 \mathrm{~m}\right)$ und befanden sich auf einer Hangneigung von $22^{\circ}$. Am unteren Ende der Messparzelle wurde jeweils eine Grube mit einer Größe von $0,6 * 0,8 * 5 \mathrm{~m}$ ausgehoben, die mit Kunststofffolie ausgelegt wurde. Die Folie wurde perforiert, damit das Wasser aus der Grube ablaufen konnte. Um am Hang herabfließendes Fremdwasser abzuleiten, zog man oberhalb der Messanlage einen Abflussgraben.

Das in den Erosionsmessanlagen angesammelte Kolluvium wurde mindestens zweimal monatlich oder nach jedem Starkregenereignis gemessen und beprobt (eine Mischprobe aus zehn Einzelproben).

Die Analyse der entnommenen Bodenproben fand dann am Geographischen Institut der Universität Göttingen statt.

\section{Erfassung von Niederschlagsparametern}

Die Messung der Niederschlagsmenge und der Niederschlagsintensität erfolgte mit einem Niederschlagsschreiber und einer einfachen Gefäßmessung. Aus finanziellen Gründen konnte für die vier Messparzellen nur ein einziger Niederschlagsschreiber eingerichtet werden. Dieser liegt auf 570 m ü. NN SW. Der Niederschlagsschreiber wurde in 1m Höhe 
über der Geländeoberfläche aufgestellt. Zur Ergänzung der Ergebnisse wurde außerdem täglich zweimal (7 Uhr und 19 Uhr) die Regenmenge am Gefäßmesser abgelesen. Es wurden außerdem die Niederschlagsdaten der Wetterstation des Babe Distrikts auf $560 \mathrm{~m}$ NN verwendet. Die Wetterstation befindet sich $6 \mathrm{~km}$ südöstlich der Messparzellen.

\section{Bodenbedeckungsgrad und Trockenmasse der Vegetation:}

Am Anfang der Erosionsmessung fand die Erfassung der Bodenbedeckung statt. Erfasst wurde die direkte Bedeckung der Bodenoberfläche durch Kulturpflanzen (z.B. Mais), Wildkräuter und Gras in verschiedenen Entwicklungsstadien. Ebenso wurden die Mulchund Humusauflage sowie Litter aus Sekundärwald- oder Ernterückständen mit einem Messstab gemessen und visuell geschätzt. Die Bestimmung der Bodenbedeckung der einzelnen Komponenten sowie des Wachstums der Kulturpflanzen erfolgte alle zwei Wochen. Zusätzlich wurde der Kronenschlussgrad von Tephrosia Candida ermittelt.

Der Bedeckungsgrad wurde pro Testfläche in verschiedenen Zeiträumen fotographisch erfasst und anschließend der prozentuale Anteil der bedeckten Bodenoberfläche auf dem jeweiligen Dia visuell ermittelt. Als Orientierung dienten die Vergleichsbilder zur Abschätzung des Bedeckungsgrades der Bodenoberfläche durch ackerbauliche Kulturen des DVWK(1996).

Zur Erfassung der Biomasse auf den Parzellen wurden die Kulturpflanzen, die Wildkräuter und der vollständige Sekundärwaldbestand auf einer Fläche von $1 \mathrm{~m}^{2}$ abgeerntet. Sowohl das Frischgewicht als auch das Trockengewicht wurde festgehalten. Ebenso wurde mit der Mulchbedeckung verfahren.

Zur Beobachtung der Maispflanzenentwicklung und des Maisertrags wurden als Kenndaten die Länge und der Durchmesser der Maiskolben [cm], die Zahl der Maiskörner pro Reihe und der tatsächliche Gesamtmaisertrag gemessen.

Außerdem Beobachtung der Wachstum von Unkraut, Tephrosia Candida und Leuceana glauca

\subsection{Methodik der Laboranalytik}

Nach der Bodenbeprobung auf den Untersuchungsflächen wurden die insgesamt 90 Einzelproben im Labor der Thainguyen Universität an der Luft getrocknet, mit $2 \mathrm{~mm}$ Maschenweite gesiebt und für den Transport vorbereitet. Alle weiteren Analysen erfolgten nach dem Flugzeugtransport aus Vietnam nach Deutschland jeweils an einem Probenaliquot von ca. $200 \mathrm{~g}$ in den Laboratorien der Abteilung Landschaftsökologie des Geographischen Instituts der Universität Göttingen. 


\section{Bodenfarbe}

Die Bestimmung der Bodenfarbe erfolgte anhand der MUNSELL SOIL COLOUR CHARTS (1905). Nach der Bestimmung im Feld wurden die Proben im Labor luftgetrocknet und es erfolgte eine weitere Farbbestimmung.

\section{Korngrößenverteilung, Bodenart, Textur}

Die Bodenart wurde nach dem kombinierten Sieb- und Pipettverfahren gemäß KÖHN (1928) ermittelt „DIN 19683 (1973) “ jetzt „IS0 11277 (2002)“. Vor der Nasssiebung wurde die organische Substanz des Bodens mit 30 \%igem Wasserstoffperoxid aufoxidiert, Ausschließend wurde die humusfreie Feinbodensubstanz mit Natriumpyrophosphat $(0,4 \mathrm{~N})$ dispergiert. Nach der Trockensiebung wurden die Sandfraktionen gravimetrisch ermittelt. Die Schlufffraktionen sowie der Ton wurden während der Sedimentation pipettiert und gravimetrisch quantifiziert.

Mit den Ergebnissen des kombinierten Sieb- und Pipettverfahrens konnte die Bestimmung der Bodenart nach dem Bodenartendiagramm der AG BODEN (1994) sowie demselbigen der U.S. SOIL TAXONOMY (1998) vorgenommen werden.

\section{Austauschbare Kationen, effektive Kationenaustauschkapazität (KAKeff)}

Zur Bestimmung der austauschbaren Kationen wurde das Perkolationsverfahren nach nach MAIWES et al. (1984) angewendet und geändert durch LÜER UND BÖHMER (2000). 2,5 g. von Boden $(<2 \mathrm{~mm})$ mit $100 \mathrm{ml}$ [ 1M ] NH4Cl Lösung. Von den Extrakten wurde $\mathrm{H}+$ potentiometrisch und $\mathrm{Al}, \mathrm{Ca}, \mathrm{Fe}, \mathrm{K}, \mathrm{Mg}, \mathrm{Mn}, \mathrm{Na}$ über ICP- OES gemessen.

\section{Gesamtkohlenstoff, Gesamtstickstoff und Humusgehalt}

Die Bestimmung von $\mathrm{Ct}$ und Nt erfolgte am CHN- Analysator 1000 der Fa. LECO. Hierbei wird das ofentrockene Probengut in reiner Sauerstoffatmosphäre verbrannt, bevor Kohlenstoff mittels einer Infrarotmesszelle und Stickstoff mittels Wärmeleitfähigkeitszelle gemessen werden.

Über die Bestimmung des Gesamtkohlenstoffs mittels Verbrennung in reiner Sauerstoffatmosphäre lassen sich nach SCHACHTSCHABEL (1992) die Humusgehalte von Böden rechnerisch ermitteln. Bei einem durchschnittlichen Kohlenstoffgehalt von 58\% im Humus der Ackerböden ergibt sich ein Umrechnungsfaktor von 1,72 bzw.:

Humus $(\%)=$ organischer Kohlenstoff Corg. $(\%) * 1,72$ 


\section{pH- Werte}

Die pH- Werte der Böden wurden in KCL- Lösung nach MEIwES et al. (1984) und $\mathrm{H}_{2} 0$ Lösungen nach VDLUFA(1991) bestimmt. Die Lösungen wurden im Verhältnis 1:2,5 mit destilliertem Wasser und $1 \mathrm{M} \mathrm{KCL}$ angesetzt und nach einer Stunde potentiometrisch am pH-Meter INOLAB Level 3 der Fa. WTW mittels Glaselektrode (SENTIX 61) gemessen.

\section{Phosporbestimmung(nach Bray)}

Das pflanzenverfügbare Phosphor wurde durch Extraktion mit $\mathrm{NH}_{4} \mathrm{~F}$ und $\mathrm{HCl}$ (nach BRAY AND KURZ 1945) bestimmt.

Zur Herstellung von 0,5 1 Extraktionslösung werden $15 \mathrm{ml} 1 \mathrm{~N} \mathrm{NH} \mathrm{NH}_{4} \mathrm{~F}$ und $25 \mathrm{ml} \mathrm{0,5} \mathrm{N} \mathrm{HCl}$ gemischt und mit aqua dest. aufgefüllt (vgl. BRAY und KURTZ 1945, PAGE 1982, SoIL SURVEY LABORATORY STAFF 1992, ISRIC 1992). Ferner wird durch Mischung von $1 \mathrm{ml}$ $\mathrm{SnCl}_{2}$ - Stammlösung (10g SnCl 2 in $25 \mathrm{ml}$ aqua dest. lösen) mit $333 \mathrm{ml}$ aqua dest. (kurz vor Gebrauch herstellen) eine Verdünnungslösung gewonnen und durch Lösen von $15 \mathrm{~g}$ Ammoniumheptamolybdat $\left(\left(\mathrm{NH}_{4}\right)_{6} \mathrm{Mo}_{7} \mathrm{O}_{24} * 4 \mathrm{H}_{2} \mathrm{O}\right)$ in $350 \mathrm{ml}$ aqua dest. mit anschießender Zugabe von $350 \mathrm{ml}$ 10N HCl sowie abermaliger Zugabe von $350 \mathrm{ml}$ aqua dest. eine Färbelösung hergestellt.

$\mathrm{Zu} 1 \mathrm{~g}$ ungemahlenem Boden wurden 7ml Extraktionslösung gegeben, der Behälter $1 \mathrm{~min}$ geschüttelt und die Probe filtriert. $2 \mathrm{ml}$ des Extraktes wurden mit $5 \mathrm{ml}$ aqua dest., $2 \mathrm{ml}$ Färbelösung und $1 \mathrm{ml}$ Verdünnungslösung aufgefüllt. Nach frühestens 5 und spätestens 18 min wurde am Spektralphotometer ( Lamda 40 von PERKIN ELMER) bei $660 \mathrm{~nm}$ die Extinktion gemesen.

\section{Totalgehalte: N, K, Ca, AL, Mg, Fe, Cu und Mn:}

Nach der von RUPPERT(1987) beschriebenen Druckaufschlussmethode wurden die Proben mit den Säuren HNO3 und HF unter hohem Druck und Energiezufuhr in der PICOTRACE- Apparatur bei $180^{\circ}$ aufgeschlossen. Die resultierenden Aufschlusslösungen wurden am ICP-OES Optima 4300DV von PERKIN ELMER gemessen.

\section{Bestimmung des Feuchtigkeitsgehaltes der Böden}

Die Bestimmung der Feuchtigkeit erfolgte nach DiN 4016 im Trockenschrank bei $105^{\circ} \mathrm{C}$ und wurde nach der Gleichung: $\mathrm{W}_{\mathrm{n}} \%=\mathrm{G}_{\mathrm{w}} \cdot 100 / \mathrm{G}_{\mathrm{t}}$

Berechnet, wobei $W_{n}$ der natürliche Wassergehalt, $G_{w}$ das Gewicht des Wassers und $G_{t}$ das Gewicht der trockenen Probe bedeutet. Die angegebenen Werte entsprechen den Mittelwerten von 5 Parallelproben. 


\subsection{Bewertungskriterien der Böden}

Um das landwirtschaftliche Bodenpotential einer Fläche aus den ermittelten Ergebnissen heraus bewerten zu können, werden verschiedene Richtwerte, Klassifizierungen, allgemeingültige Grenzwerte und Bewertungsmaßstäbe für die Beurteilung von einzelnen bodenphysikalischen und- chemischen Parameter herangezogen. Die Tab 7 bis 11 zeigen die verwendeten Richt- und Grenzwerte nach verschiedenen Autoren .

Diese Werte zeigen jedoch nur Tendenzen auf, denn abhängig von der verwendeteten Methodik für die einzelnen Parameter sind unterschiedliche Ergebnisse festzustellen. Ebenso sagt der Vergleich innerhalb der verschiedenen Böden nicht aus, ob die einzelne Pflanze ausreichend mit Nährstoffen versorgt ist.

Tab. 7 : Richtwerte für die Beurteilung der KAKeff nach LANDON 1991

\begin{tabular}{lrrrrr}
\hline Niveau & KAKeff & $\begin{array}{c}\mathrm{Ca}(\mathrm{cmol} / \mathrm{kg} \\
\text { Boden })\end{array}$ & $\begin{array}{c}\mathrm{Mg}(\mathrm{cmol} / \mathrm{kg} \\
\text { Boden })\end{array}$ & $\begin{array}{r}\mathrm{K}(\mathrm{cmol} / \mathrm{kg} \\
\text { Boden })\end{array}$ & $\begin{array}{c}\mathrm{Na}(\mathrm{cmol} / \mathrm{kg} \\
\text { Boden })\end{array}$ \\
\hline sehr gering & $<5$ & $<2,0$ & $<0,5$ & $<0,10$ & $<0,05$ \\
\hline gering & $5-15$ & $2,1-5,0$ & $0,5-1,5$ & $0,11-0,20$ & $0,05-0,20$ \\
\hline mittel & $15-25$ & $5,1-10,0$ & $1,6-4,0$ & $0,21-0,50$ & $0,21-0,70$ \\
\hline hoch & $25-40$ & $10,1-20,0$ & $4,1-8,0$ & $0,51-1,20$ & $0,71-2,00$ \\
\hline sehr hoch & $>40$ & $>20,0$ & $>8,0$ & $>1,2$ & $>2,01$ \\
\hline
\end{tabular}

Tab. 8 : Richtwerte für die organische Substanz und den Gesamtgehalt an Stickstoff für die verschiedenen Bodenarten nach Pagel 1982

\begin{tabular}{lrrrrrrr}
\hline Bewertung & \multicolumn{4}{c}{ Humusgehalt (\%) } & & $\mathrm{Nt}(\%)$ & \multicolumn{2}{c}{$\begin{array}{c}\mathrm{N} / \mathrm{N} \\
\end{array}$} & $\begin{array}{c}\text { Sand- } \\
\text { böden }\end{array}$ & $\begin{array}{c}\text { Lehm- } \\
\text { böden }\end{array}$ & $\begin{array}{c}\text { Ton- } \\
\text { böden }\end{array}$ & $\begin{array}{c}\text { Sand- } \\
\text { böden }\end{array}$ & $\begin{array}{c}\text { Lehm- } \\
\text { böden }\end{array}$ & $\begin{array}{c}\text { Ton- } \\
\text { böden }\end{array}$ & Ratio \\
\hline Sehr gering & $<0,5$ & $<1,0$ & $<1,5$ & $<0,025$ & $<0,05$ & $<0,075$ & $>25$ \\
\hline Gering & $0,5-1,0$ & $1,0-1,5$ & $1,5-2,0$ & $0,025-0,05$ & $0,05-0,075$ & $0,075-0,1$ & $25-20$ \\
\hline Mäßig & $1,0-1,5$ & $1,5-3,0$ & $2,0-4,0$ & $0,05-0,075$ & $0,075-0,15$ & $0,1-0,2$ & $20-15$ \\
\hline Hoch & $1,5-3,0$ & $3,0-5,0$ & $4,0-8,0$ & $0,075-0,15$ & $0,15-0,25$ & $0.2-0,4$ & $15-10$ \\
\hline Sehr hoch & $>3,0$ & $>5,0$ & $>8,0$ & $>0,15$ & $>0,25$ & $>0,4$ & $<10$ \\
\hline
\end{tabular}

Tab.9 : Richtwerte für pflanzenverfügbare Nähstoffe und den Gesamtgehalt an Schwefel nach LANDON 1984

\begin{tabular}{|c|c|c|c|c|c|c|c|}
\hline Bewertung & $\mathrm{P}(\mathrm{mg} / \mathrm{kg})$ & $\mathrm{K}(\mathrm{mg} / \mathrm{kg})$ & $\mathrm{Ca}(\mathrm{mg} / \mathrm{kg})$ & $\mathrm{Mg}(\mathrm{mg} / \mathrm{kg})$ & $\mathrm{Ca} / \mathrm{Mg}$ & $\mathrm{Mg} / \mathrm{K}$ & St \\
\hline Gering & $<5$ & $<80$ & $<800$ & $<60$ & $<3$ & $<0,5$ & $<200$ \\
\hline Mittel & $5-15$ & $80-230$ & $800-2000$ & $60-500$ & $3-5$ & & \\
\hline Hoch & $>15$ & $>230$ & $>2000$ & $>500$ & $>5$ & & \\
\hline
\end{tabular}


Tab. 10 : Richtwerte für die Beurteilung der Sättigungsgrade an der KAK in \% nach PAGEL 1982

\begin{tabular}{lrrrr}
\hline Kennzeichnung & \multicolumn{5}{c}{ Sättigungsgrade an der KAK in \% } \\
\cline { 2 - 5 } & $\mathrm{V}_{\mathrm{Ca}}$ & \multicolumn{4}{c}{$\mathrm{V}_{\mathrm{Mg}}$} & $\mathrm{V}_{\mathrm{K}}$ & \multicolumn{1}{c}{$\mathrm{V}_{\mathrm{Na}}$} \\
\hline Sehr gering & $<5$ & $<2$ & $<0,5$ & $<0,1$ \\
\hline Gering & $5-20$ & $2-10$ & $0,5-1$ & $0,1-2$ \\
\hline Mittel & $20-60$ & $10-25$ & $1-2,5$ & $2-10$ \\
\hline Hoch & $60-90$ & $25-40$ & $2,5-10$ & $10-25$ \\
\hline Extrem hoch & $>90$ & $>40$ & $>10$ & $>25$ \\
\hline
\end{tabular}

Tab. 11: Richtwerte für Phosphor pflanzenverfügbar nach HofFMANN (1991):VDLUFA- Verlag

\begin{tabular}{lllcc}
\hline $\mathrm{P}$ & $<3 \mathrm{ppm}$ & $3-7 \mathrm{ppm}$ & $7-20 \mathrm{ppm}$ & $>20 \mathrm{ppm}$ \\
\hline Bewertung & sehr geringe & gering & mittel & hoch \\
\hline
\end{tabular}

\subsection{Statistische Auswertung}

Im Vorfeld der Untersuchung wurde darauf geachtet, eine genügend große Anzahl an Stichproben zu erheben, um im Anschluss eine statistische Auswertung gewährleisten zu können.

Die statistischen Analyse erfolgten mit dem Programmpaket SPSS/PC (Vergleich von mehr als zwei unabhängigen Stichproben nach KRUSKAL und WALLIS in BÜHL \& ZÖFEL (1996) und MICROSOFT EXCEL. Um die Stärke des Zusammenhangs zwischen zwei Variablen zu beschreiben, wurde mittels des PEARSONschen Korrelationskoeffizienten der lineare Zusammenhang zwischen zwei Variablen untersucht. Die einfache Regressionsanalyse ermöglichte es, diese Beziehung zwischen einer abhängigen und unabhängigen Variabeln in einer Gleichung wiederzugeben: $Y=a+b x$

Wobei: $\mathrm{Y}=$ abhängige Variable; $\mathrm{b}=$ Regressionskoeffizient; $\mathrm{a}=$ konstantes Glied der Regressionsfunktion; $\mathrm{x}=$ unabhängige Variable, $\mathrm{R}=$ Korrelationsgrad

Zur statistischen Beschreibung wurden ferner das Minimum und Maximum hinzugezogen, sowie eine klassifizierte Häufigkeitsverteilung und ihre Schiefe. Die Variablen der Datensätze für die Regressionsanalyse wurden auf Orthogonalität der Einflussgrößen untereinander und die Signifikanz der Prädiktoren ( Student'scher T-Test, F-Test, Konfidenzintervall von 95\%, 99\%) getestet. 


\section{SOZIOÖKONOMISCHE ANALYSE DER LANDWIRTSCHAFTLICHEN HAUSHALTE}

„Armut ist nicht Gott-gegeben. Armut kann durch gemeinsame Anstrengungen beseitigt werden. Die Strategie muss alle Sektoren und Ebenen unserer Gesellschaft umfassen, vor allem müssen dabei der Staat, der Markt sowie die Zivilgesellschaft mit eingeschlossen werden." Ela Bhatt ${ }^{1}$.

Um die sozioökonomische Lage des Untersuchungsgebiets $\mathrm{zu}$ ermitteln, wurden Erhebungen durch Interviews mit Betriebsleitern in $\mathrm{BaBe}$ hinsichtlich der Wirtschaftssituation, der Familienstruktur, der Bildung und der natürlichen bzw. menschlichen Ressourcen durchgeführt und analysiert. Im Folgenden werden Ergebnisse, basierend auf den genannten Interviews, dargestellt. Die Analyse stellt sich als Hintergrund für die im Kapital 6 ,Analyse von angepassten Anbausystemen und Bodenerosionsschutzmaßnahmen“ dar.

\subsection{Klassifizierung und Auswahl von Haushalten für die Fragebogen - Erhebung}

Um die Lebensverhältnisse der lokalen Bevölkerung zu erfassen, wurden 45 Haushalte mit einem standardisierten Fragebogen interviewt. Die befragten Personen wurden nach dem Index der Klassifizierung von Haushalten der Dorfverwaltung ausgewählt.

Es handelt sich dabei um verschiedene Merkmale wie die wirtschaftlichen Verhältnisse, die Struktur der Familien, die verfügbaren Arbeitskräfte, die natürliche Ressourcen, wie z. B. Landnutzungsfläche, Wald oder Art und Menge der Viehhaltung

Die Klassifizierung des Lebensstandards der Bevölkerung basiert auf dem tatsächlichen Besitztum der Bauern: Eigenhaus und dessen Größe bzw. Beständigkeit, Anzahl der Rinder/Büffel und Schweine sowie die verfügbaren Lebensmittel. Dabei ist die Wertskala "reich", "mittelständisch", "arm" und "sehr arm" relativ. Sie soll das Verhältnis des Lebensniveaus der lokalen Bevölkerung nur plastisch darstellen.

\footnotetext{
1 : Trägerin des Alternativen Nobelpreises, Gründerin von SEWA, Indien (Self Employed Women's Association)
} 
Tab.12: Wirtschaftliche Lage der Bevölkerung von Dia Linh im Jahre 2001 (Lebensstandard)

\begin{tabular}{|l|c|c|c|c|}
\hline $\begin{array}{l}\text { Merkmale des } \\
\text { Lebensstandards }\end{array}$ & reiche Familie & $\begin{array}{c}\text { mittelständische } \\
\text { Familie }\end{array}$ & arme Familie & $\begin{array}{c}\text { sehr arme } \\
\text { Familie }\end{array}$ \\
\hline $\begin{array}{l}\text { Größe und } \\
\text { Dauerhaftigkeit } \\
\text { des Hauses }\end{array}$ & $\begin{array}{c}\text { großes Haus mit } \\
\text { hoher } \\
\text { Dauerhaftigkeit }\end{array}$ & $\begin{array}{c}\text { großes Haus mit } \\
\text { geringer } \\
\text { Dauerhaftigkeit }\end{array}$ & $\begin{array}{c}\text { kleines Haus mit } \\
\text { geringer } \\
\text { Dauerhaftigkeit }\end{array}$ & $\begin{array}{c}\text { kleines und } \\
\text { kurzlebiges Haus }\end{array}$ \\
\hline $\begin{array}{l}\text { Anzahl der } \\
\text { Rinder/ Büffel } \\
\text { (Tiere) }\end{array}$ & $>7$ & $3-6$ & $1-2$ & 0 \\
\hline $\begin{array}{l}\text { Anzahl der } \\
\text { Schweine (Tiere) }\end{array}$ & $>10$ & $5-9$ & $3-4$ & $1-2$ \\
\hline $\begin{array}{l}\text { Verfügbarkeit von } \\
\text { Lebensmitteln }\end{array}$ & $\begin{array}{c}\text { Überschüsse } \\
\text { erzielend }\end{array}$ & Bedarf deckend & nicht ausreichend \\
für 3-6 Monate & nicht ausreichend \\
für > 6 Monate
\end{tabular}

(Daten aus: Verwaltung Dia Linh Dorf)

Die obige Tabelle dokumentiert, dass reiche Familien über mehr Besitz verfügen als arme Familien. Sie können sich ein großräumiges, festes und dauerhaftes Haus leisten. Außerdem gehören ihnen viele Büffel und/oder Rinder, die eine sehr wichtige Rolle bei der Bodenbearbeitung eigener Anbauflächen spielen. Die Büffel können zudem an arme Familien vermietet werden. Als Gegenleistung erhalten die Besitzer landwirtschaftliche Produkte.

Die Anbauflächen der reichen Familien sind im Normalfall groß und mit guten Bewässerungsmöglichkeiten ausgestattet.

Bei den armen Familien mangelt es v.a. an Finanzreserven und Produktionsmitteln. Ihre Anbauflächen sind in der Regel klein, von schlechter Bodenqualität und befinden sich in Höhenlagen mit Hangneigungen von teilweise mehr als $20^{\circ}$. Sie können deshalb noch nicht einmal genügend Reis für ihren Eigenbedarf anbauen. Es fehlt ihnen das Geld für nötige Investitionen zur Verbesserung der Böden und zur Beschaffung von hochwertigerem Saatgut und anderen Produktionsmitteln, so dass die Böden bereits nach kurzer Zeit erodieren und verarmen.

\subsection{Ressourcen und ihre Nutzung}

Die vielfältigen Ressourcen - seien es menschliche Arbeitskräfte oder natürliche Ressourcen - beeinflussen einerseits direkt das Leben der Menschen im Dia Linh, 
andererseits bestimmt auch die Art und Weise ihrer Nutzung entscheidend die wirtschaftliche sowie die ökologische Entwicklung dieser Region mit. Ein Beispiel dafür ist die Ausbeutung der Wälder im Rahmen der Ausdehnung von Anbauflächen. Sie führte und führt zu zunehmender Bodenerosion bzw. zur Bodendegradation und schließlich zur Minderung der Produktivität der Bewirtschaftungsfläche und damit zur Verhinderung wirtschaftlicher Entwicklung des Dorfes.

\subsubsection{Menschliche Ressourcen}

Menschen spielen eine sehr wichtige Rolle in der Ressourcennutzung. Faktoren wie die Bevölkerungsentwicklung, die Familienstruktur, das Bildungsniveau, die geschlechtliche Arbeitsteilung, etc. bestimmen die Bedürfnisse an Nutzungsflächen sowie die Wirtschaftsweise und das Verhalten des Menschen im Umgang mit Naturressourcen.

\subsubsection{Größe und Struktur der Familie}

Hinsichtlich der durchschnittlichen Anzahl der Familienmitglieder pro Haushalt wurde mit einer statistischen Genauigkeit von 90\% (Konfidenzintervall nach KRUSKAL WALLIS, S. 280 in SPSS, 2002) kein Unterschied zwischen reichen und armen Familien festgestellt.

In Dia Linh - Ba Be leben in einem Haushalt meistens drei Generationen zusammen. In den „reichen“ Familien leben mehr Männer als Frauen, dagegen leben bei den „armen“ Familien mehr Frauen als Männer, wie Tabelle 13 gezeigt hat. Der Anteil der Männer in reichen Familien beträgt 69,6\%, in armen Familien jedoch nur 62,2\%.

Die Anteile von Personen, die jünger als 14 Jahre sowie älter als 60 Jahre und somit hilfsbedürftig und abhängig sind, sind in den armen Familien mit 37,8\% größer als in den reichen Familien mit 30,4\%. Diese Struktur verdeutlicht, dass arme Familien in schlechteren Lebensverhältnissen leben. Sie verfügen über nur kleine Anbauflächen in ungünstigsten geographischen Lagen, über weniger kräftige Arbeitskräfte (also Männer) und müssen im Vergleich zu den reichen Familien mehr Menschen - darunter mehr Hilfedürftige - ernähren.

Der Anteil von Kindern unter 14 Jahren liegt bei den armen Familien mit 37,7\% deutlich höher als bei der reichen Familien mit 30,4\%. Im Vergleich zu den Familien mit nur zwei Kindern, wie sie etwa in den Deltagebieten oder in den Städten leben, sind die Familien in Dia Linh - Ba Be im allgemeinen kinderreich (nach dem Familiengesetz von Vietnam darf jede Familie 2 Kinder haben). Die Anzahl der Familiemitglieder sowie die kleinen 
Anbauflächen sind zwei der Gründe, die dazu führen, dass die Anbaufläche pro Person sehr intensiv genutzt und die Brachperiode somit verkürzt wird. Die Folge sind schwerwiegende Bodendegradationen.

Tab. 13. Durchschnittliche Anzahl der Personen pro Familie in Dia Linh - Ba Be, 2001

\begin{tabular}{lccr}
\hline \multicolumn{1}{c}{ Struktur der Familie } & $\begin{array}{c}\text { Reich } \\
(\boldsymbol{n}=\mathbf{1 1})\end{array}$ & $\begin{array}{c}\text { Mittelständisch } \\
(\boldsymbol{n}=\mathbf{2 3})\end{array}$ & $\begin{array}{c}\text { Arm } \\
(\boldsymbol{n}=\mathbf{1 1})\end{array}$ \\
\hline Familienmitglieder & 7,18 & 5,91 & 5,27 \\
& $(0,91)$ & $(0,75)$ & $(1,02)$ \\
\hline $\begin{array}{l}\text { Davon: } \\
\text { Anteil von }\end{array}$ & & & \\
$\begin{array}{l}\text { Männern (in \%) } \\
\text { und }\end{array}$ & 69,64 & 67,01 & \\
Kindern unter 14 Jahren (in \%) & 30,36 & 32,99 & 37,76 \\
\hline $\begin{array}{l}\text { Werte in Klammer: geschätzter Mittelwert }(\alpha=0.1) \\
\text { Nach: KRUSKAL WALLIS (Familienmitglieder) Test: Chi-Square }=4,276\end{array}$ & \multicolumn{2}{c}{ Asymp. Sig $=0,118$}
\end{tabular}

Bildung ist ein wichtiger Faktor für die gesellschaftliche Entwicklung. Das Gleiche gilt auch für die Förderung des Wirtschaftens. Wissen erleichtert den Menschen das Erlernen und die Aneignung von neuen Verfahren und Techniken bzw. Landnutzungssystemen zur Erhöhung der Produktivität der Bodennutzung und zur Schonung von Naturressourcen. Außerdem hilft es den Menschen, gesetzliche Bestimmungen $\mathrm{zu}$ begreifen und durchzuführen.

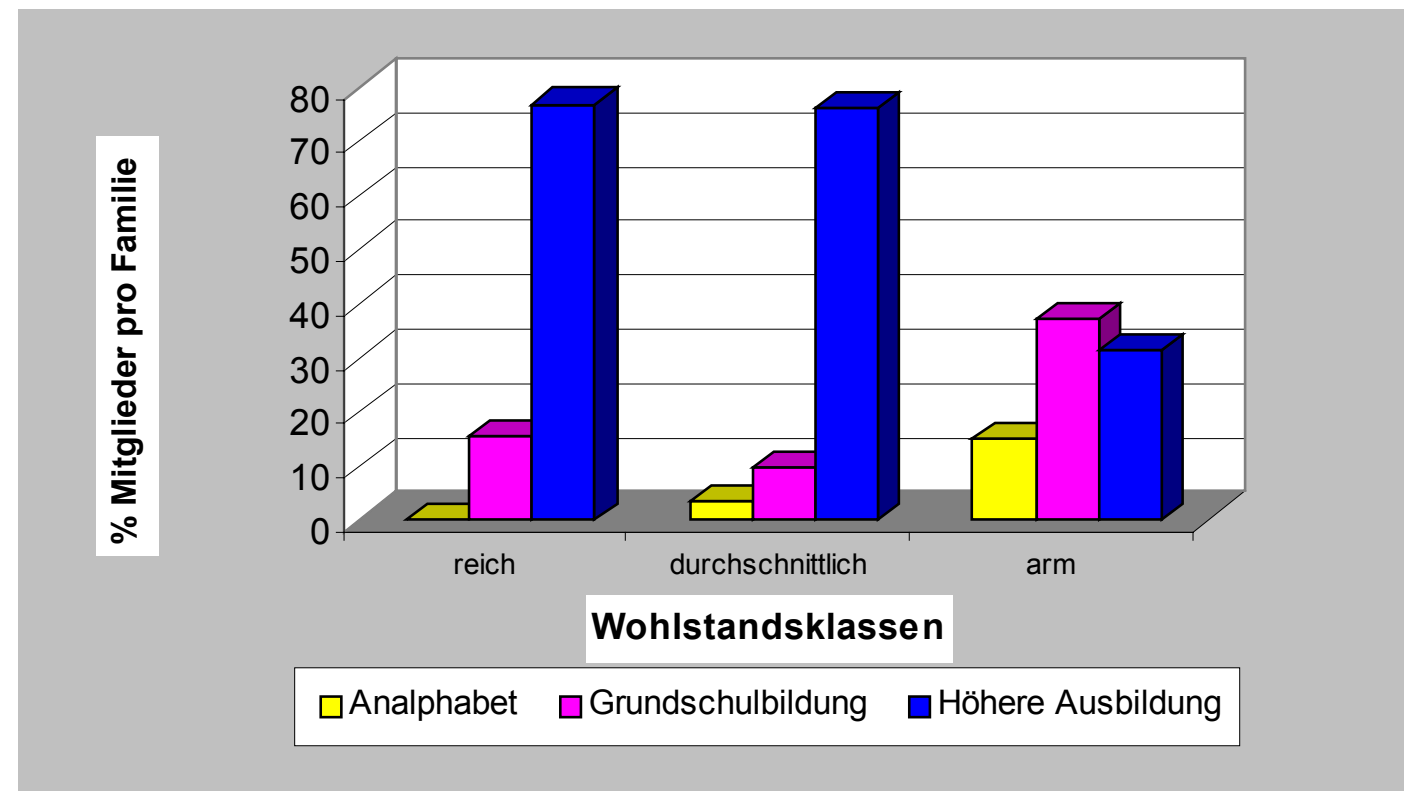

Abb.10. Bildungsniveau der Familien im Untersuchungsgebiet

Quelle: Eigene Erhebung

Bei den armen Familien in Dia Linh - Ba Be ist die Analphabetenrate sehr hoch. Es fehlt hier das Geld, um die Kinder zur Schule zu schicken. Zudem werden Kinder von der 
Familie als zusätzliche Arbeitskraft behandelt. Sie werden für unterschiedliche Arbeiten eingesetzt.

Aus dem oben dargelegten Diagramm wird deutlich, dass der Anteil an Analphabeten in armen Familien 10\% höher ist als der Anteil in reichen Familien, der bei 0\% liegt. Eine höhere Ausbildung ist bei den Angehörigen der reichen Familien mit knapp 70\% wesentlich weiter verbreitet, als bei den Angehörigen der armen Familien mit nur ca. 25\%. Kinder der armen Familien haben also geringere Chancen auf eine höhere Ausbildung. Ein Großteil der Bevölkerung der Untersuchungsgebiete steht noch auf einem außerordentlich niedrigen Bildungsniveau. Solange das Analphabetentum nicht überwunden wird, ist es schwer, technischen Fortschritten in die Landwirtschaft Eingang zu verschaffen. Masseninformationsmittel, wie Fachzeitschriften, Fernsehen, sind kaum vorhanden. Die Wirtschaftsberatung ist wenig entwickelt. Die allgemeine Bildung und ihr fachliches Wissen - hier die Anbaumethoden - sind sehr eingeschränkt.

\section{Armut und Umweltzerstörung - ein Teufelskreis}

Es scheint ein Teufelskreis zu sein: Umweltzerstörung macht die Menschen arm, und Armut führt zu Umweltzerstörung. Wo die Menschen keinen Zugang zu natürlichen Ressourcen - etwa zu fruchtbarem Boden, Wasser oder Brennholz - haben, verschärft sich die Armut. Andererseits zwingt Not die Menschen oft dazu, ihre Umwelt massiv zu schädigen. Ist beispielsweise fruchtbarer Boden knapp oder sind die Nutzungsrechte für gute Böden ungleich verteilt, werden arme Bevölkerungsgruppen dazu getrieben, karge Gebiete zu bebauen, die dieser Belastung eigentlich nicht gewachsen sind. Das führt zu „Übernutzung“, wie die Fachleute es nennen: Der Boden wird so ausgelaugt, dass nach einiger Zeit noch weniger wächst als zuvor. Ergebnis: Die Armen werden ärmer - und müssen auf noch schlechtere Böden ausweichen. Ein Teufelskreis, der ganze Landstriche veröden und endgültig unfruchtbar werden lässt und die Menschen aus ihrer Heimat vertreibt. 


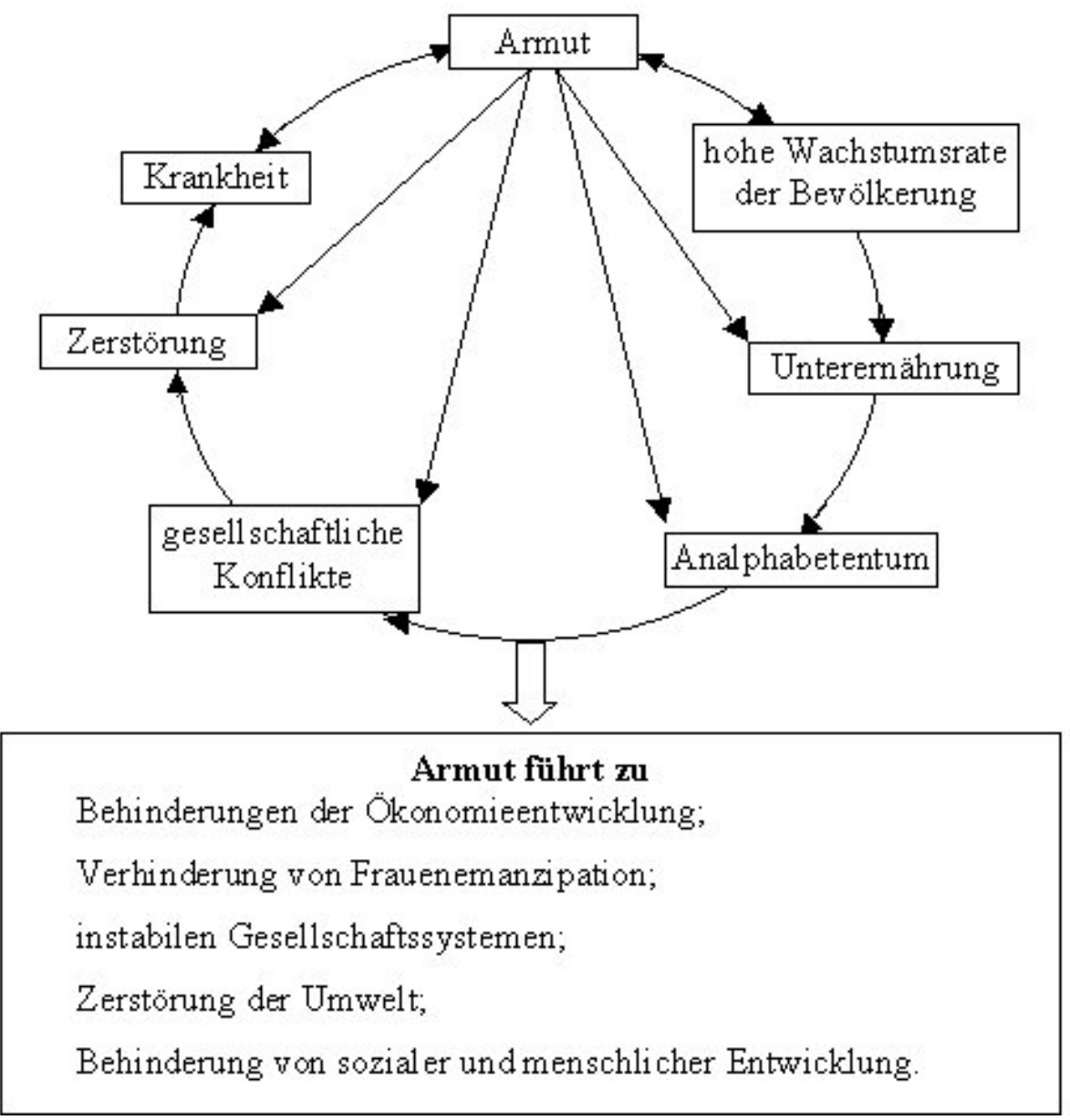

Quelle: Vu TUYEN HOANG, 2001

Abb.11: Der Teufelkreis der Armut

Armut und Überbevölkerung werden neben dem industriellen Raubbau als die hauptverantwortlichen Ursache für die Umweltzerstörung in den Entwicklungsländern gesehen. Der zunehmende Bevölkerungsdruck zwingt dazu, das Land immer intensiver zu nutzen oder die Agrikultur auch auf ärmere und vor allem steilere Böden auszudehnen, die bei uns normalerweise der Forstwirtschaft oder dem Naturschutzgebieten auf landwirtschaftlich uninteressanten Flächen vorbehalten ist. Marginalisierte Böden und die durch die Überbevölkerung angespannte Brennholzsituation sorgt für eine ausgeräumte Landschaft (BMZ- Zeitschrift, 2002).

\subsubsection{Arbeitskräfte}

Die Arbeitsverfassung regelt die Beziehungen zwischen den die Landarbeit verrichtenden Menschen. Schon immer setzten Angehörige einer Familie ihre gemeinsamen Kräfte zur Deckung ihrer Bedürfnisse ein. Die Familienarbeitsverfassung ist nicht nur altbewährt, sondern auch weitverbreitet. Jedes Mitglied wird durch die Sicherheit, dass es das Ergebnis seiner Anstrengungen nur mit Mitgliedern der eigenen Sozialgruppe Familie 
teilen wird, zu Leistung angeregt. Die jeweilige, im Lebenszyklus der Familie wechselnde Arbeitskapazität, beeinflusst die Betriebsorganisation. Engpässe werden durch Überbelastung, Änderung der Intensität oder Zu- bzw. Verpachtungen ausgeglichen.

Probleme für die Familienarbeitsverfassung können entstehen, wenn die Betriebseinheit zu klein wird und nicht mehr alle Angehörige für die Arbeit benötigt werden. Solange keine alternativen Existenzmöglichkeiten bestehen, hält die Familie zusammen und teilt sich die vorhandene Arbeit mit den dazugehörigen Erträgen. Das führt zum Zustand der ländlichen Unterbeschäftigung mit einem niedrigen Lebensniveau. Sobald alternative Arbeitsmöglichkeiten entstehen, kommt es zum Übergang mit Zuerwerbs- und Nebenerwerbslandwirtschaft.

Tab.14: Durchschnittliche Anzahl der Arbeitkräfte, differenziert nach Lebensstandard, Dia Linh 2001

\begin{tabular}{lc}
\hline Lebensstandard & Arbeitskräfte \\
\hline Reich $(\mathrm{n}=11)$ & $5,7(0,75)$ \\
\hline Mittel $(\mathrm{n}=23)$ & $4,6(0,60)$ \\
\hline Arm $(\mathrm{n}=11)$ & $3,9(0,96)$ \\
\hline
\end{tabular}

Der Wert in Klammern ist das Konfidenzintervall der Schätzung des Mittelwertes $(\alpha=0.1)$ nach KRUSKAL WALLIS (Arbeitskräfte) Test Chi-Square $=6,035$ Asymp. Sig $=0,049$

Das Ergebnis des Fragebogens verdeutlicht die Unterschiede, die in Bezug auf die Anzahl der Arbeitskräfte bestehen.

Ein reicher Betrieb ist mit etwa 5,7 Arbeitskräften, ein armer dagegen nur mit etwa 3,9 Arbeitskräften ausgestattet. Dabei werden Kinder nur in begrenztem Umfang als Arbeitskräfte eingesetzt, ebenso wie Personen über 60 Jahre. Zwei Drittel der reichen Familien und die Hälfte der Betriebe von armen Familien weisen einen periodischen, etwa vier Monate im Jahr auftretenden Arbeitskräftemangel auf. Dieser ist insbesondere in den Monaten Januar und Februar, in denen die Flächen gesäubert und die Böden bearbeitet, sowie im Juli und August, zur Zeit der Ernte von Mais und Trockenreis, akut.

Die Arbeitsteilung zwischen Männern und Frauen ist weitgehend ausgeglichen. Lediglich die körperlich stärker beanspruchende Bodenbearbeitung wird zu 80\% von Männern durchgeführt. Büffel und Rinder werden von Kindern nach dem Schulbesuch gehütet. Das Hüten von Büffeln wird von der lokalen Bevölkerung nicht als Arbeit sondern als spielerische Aktivität gesehen. Jede Altersgruppe übernimmt Arbeiten im Haushalt, kranke und sehr alte Personen ausgenommen. Ab einem Alter von ca. 16 Jahren sind die 
Jugendlichen dazu in der Lage, alle Arbeiten zur Aufrechterhaltung eines Haushaltes auszuführen. Das Heiratsalter der Tay beginnt i. d. R mit 18 Jahren.

Bei letzteren überwiegt der Anteil der Kinder aufgrund der hohen Geburtenrate. Die Kinder der Tay arbeiten schon früh in der Landwirtschaft mit. Der Anteil der Frauen ist gegenüber dem der Männer bei allen drei Gruppen gleich und die Lebenserwartung der Frauen ist höher als die der Männer. Bei den drei Gruppen sind die zur Verfügung stehenden Arbeitskräfte der Familien während der Arbeitsspitzen nicht ausreichend. Dies führt zu größeren Belastungen der Arbeitskräfte. Die Landwirtschaft im Bergland von Vietnam wird weitgehend zur Selbstversorgung, also in Subsistenzwirtschaft, betrieben. Je mehr Arbeitkräfte jemand zur Verfügung hat, desto mehr kann er produzieren und desto größer wird sein Besitz. Das ist auch eine der Ursachen, die zu den unterschiedlichen Lebensstandards der drei Gruppen führt.

\subsubsection{Land- und Landnutzungsressourcen}

Kap. 3.1 hat bereits gezeigt, dass zwei Drittel des Landes Hochland sind. 80\% der Bevölkerung arbeiten auf dem Land. Vietnam ist ein Land, das abhängig ist von der Landwirtschaft, den natürliche Ressourcen und dem Klima. Das Dorf Dia Linh ist eines der 2.300 ärmsten Dörfer in Vietnam .

\subsubsection{Landressourcen}

Neben der Landqualität ist auch die Landquantität ein wichtig Faktor, die die Auswahl der Kulturpflanzen mitbestimmt, die angepflanzt werden können.

Tab.15 : Aktuelle Landwirtschaftsflächen von Haushalten in Dia Linh-Ba Be, 2001

\begin{tabular}{lcrr}
\hline Fläche (ha) & $\begin{array}{c}\text { Reich } \\
(\mathrm{n}=11)\end{array}$ & $\begin{array}{c}\text { Mittel } \\
(\mathrm{n}=23)\end{array}$ & $\begin{array}{c}\text { Arme } \\
(\mathrm{n}=11)\end{array}$ \\
\hline Landwirtschaft & 2,36 & 0,99 & 0,53 \\
& $(1,14)$ & $(0,31)$ & $(0,20)$ \\
\hline Landwirtschaftliche Fläche/ & 0,30 & 0,24 & 0,13 \\
Person & $(0,12)$ & $(0,15)$ & $(0,15)$ \\
\hline
\end{tabular}

Wert in Klammern ist das Konfidenzintervall der Mittelwert Schätzung $(\alpha=0.1)$

KRUSKAL Wallis (Landwischaft) Test Chi-Square $=11,758$ Asymp. Sig $=0,03$

KRUSKAL WALLIS (landwirtschaftliche Fläche/Person) Test $\quad$ Chi-Square $=7,539$ Asymp. Sig $=0,023$

Nach KRUSKAL WALLIS erreicht die Statistik eine Genauigkeit von 95\%. 
Die durchschnittliche Betriebsgröße der reichen Familien beträgt 2,36 ha, was etwa $3.000 \mathrm{~m}^{2} /$ Person entspricht. Die durchschnittliche Betriebsgröße der armen Familien beträgt nur 0,53 ha, was etwa $1.300 \mathrm{~m}^{2} /$ Person entspricht. Ein Problem ist, dass die Flächen häufig nicht zusammenhängen, sondern in mehrere kleine Flächen zerteilt sind. Das ist besonders dann ein Problem, wenn neue Technologien eingesetzt werden soll. Die relativ geringe Flächenausstattung der Betriebe in der Untersuchungsregion ist darauf zurückzuführen, dass im Zuge der Bodenreform Landnutzungsrechte unter anderem pro Haushalt verteilt wurden. Eine künstliche Teilung der Haushalte, um mehr Nutzungsrechte zu erhalten, war als Folge zu beobachten.

Den größten Teil der Betriebsfläche nimmt in den Untersuchungsgemeinden die forstwirtschaftlich genutzte Fläche ein. Anzumerken ist dabei, dass die als forstwirtschaftlich ausgegebenen Flächen auch landwirtschaftlich genutzt werden. In der Untersuchungsregion wird auf einer Fläche von durchschnittlich 0,3- 0,5 ha pro Betrieb Brandrodungsfeldbau betrieben. Die unterschiedliche Nutzung der Betriebflächen in der Untersuchungsregion hängt mit den jeweiligen topographischen Gegebenheiten zusammen.

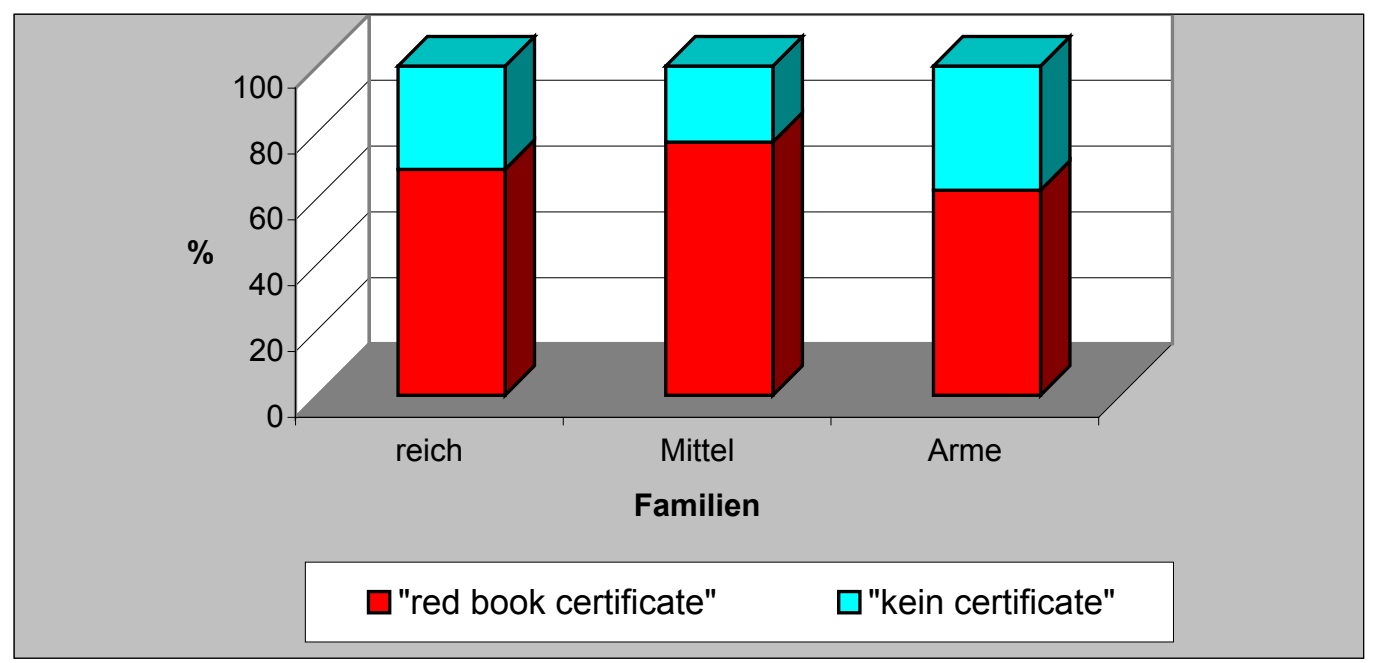

Abb.12: Situation der Landbesitzer, Dia Linh - Ba Be, 2001

Quelle: Eigene Erhebung

Vor 1986 gab es in Vietnam drei Formen des Landeigentums, das staatliche, das kollektiv-wirtschaftliche bzw. produktionsgenossenschaftliche und das private. Nach 1986 wurden die Landflächen auf Einzelhaushalte aufgeteilt. Seit das Bodengesetz 1993 überarbeitet wurde, ist es für Privatpersonen möglich, Nutzungsrechte für verschiedene Landkategorien zu erwerben. Privater Landbesitz ist untersagt, die Fläche Vietnams befindet sich nach wie vor in Staatseigentum. Die Landnutzungsrechte werden von einem 
Volkskomitee vergeben. Sie werden für einen Zeitraum von 15-20 Jahren, das sogenannte „,red book certificate“ für landwirtschaftliche Nutzfläche bzw. 30-50 Jahren, das ,,green book certificate" für forstwirtschaftlich genutzte Flächen vergeben. (LAND LAW OF VIETNAM; 1993)

$60 \%$ der landwirtschaftlich genutzten Flächen eines Durchschnittsbetriebes der Untersuchungsregionen sind mit solchen zertifizierten Nutzungsrechten ausgestattet (siehe Abb.12). Analysiert man die Allokation der Nutzungsrechte bezogen auf die jeweilige Nutzungsart der Fläche, ist festzustellen, dass fast $90 \%$ der Betriebsleiter im Dorf die bewässerten Flächen, auf denen Nassreis angebaut wird, mit dem „,red book certificate " bewirtschaften. Der hohe prozentuale Anteil der Flächenzertifizierung bei der Kulturart Reis lässt sich mit dem großen Beitrag zur Versorgung mit Grundnahrungsmitteln erklären.

Ein Problem in diesem Untersuchungsgebiet ist die Erhaltung der Bodenfruchtbarkeit, die unter anderem abhängig ist von den Erfahrungen der lokalen Bevölkerung, zu denen der Vorteil der Brache als Maßnahme, die Bodenfruchtbarkeit zu verbessern, zählt. Dieser traditionelle Wanderfeldbau ist im Hinblick auf die Bevölkerungsdichte nicht mehr angepasst. Tatsächlich werden neue technische Anbaumethoden als Alternativen zum Wanderfeldbau gefordert.

\subsubsection{Anbaustruktur}

Typische Nutzpflanzen der Tay-Bevölkerungsgruppe sind Monokulturen von Mais, Trockenreis oder Cassava im Bergland. Die Bewässerungssituation hängt vom Niederschlag ab. Die Niederschläge konzentrieren sich in der kurzen Zeit von Mai bis September. Das ist neben den Monokulturen und der starken Hangneigung eine der Ursachen für Bodenerosionen.

Reis ist in der Untersuchungsregion als Grundnahrungsmittel die wichtigste Kulturpflanzenart. $88 \%$ der Betriebe in Naduc bauen Nassreis an. Ausschließlich in diesem Dorf wird zudem von 70\% der Betriebe Trockenreis angebaut. Mais wird in allen Betrieben angebaut. Die Kulturen Soja, Gemüse und Banane werden nur im Hausgarten angebaut.

Nach Tab.16 zeigen die Formen der Bodenbearbeitung bei den drei verschiedenen Familiengruppen deutliche Unterschiede. 
Tab.16: Tatsächliche Landnutzung im Jahr 2001 im Untersuchungsgebiet

\begin{tabular}{lrrr}
\hline Kulturpflanzen & $\begin{array}{c}\text { Reiche Familien } \\
(n=11)\end{array}$ & $\begin{array}{r}\text { Mittelständische } \\
\text { Familien }(n=23)\end{array}$ & $\begin{array}{c}\text { Arme Familien } \\
(n=11)\end{array}$ \\
\hline \multirow{2}{*}{ Nassreis } & 0,44 & 0,35 & 0,13 \\
& $(0,14)$ & $(0,07)$ & $(0,02)$ \\
\hline \multirow{2}{*}{ Trockenreis } & 0,10 & 0,02 & 0,03 \\
& $(0,05)$ & $(0,02)$ & $(0,02)$ \\
\hline \multirow{2}{*}{ Mais } & 0,54 & 0,31 & 0,14 \\
& $(0,26)$ & $(0,23)$ & $(0,08)$ \\
\hline \multirow{2}{*}{ Bohne und Erdnuss } & 0,07 & 0,03 & 0,01 \\
& $(0,04)$ & $(0,02)$ & $(0,01)$ \\
Gemüse & 0,03 & 0,02 & 0,01 \\
& $(0,01)$ & $(0,01)$ & $(0,01)$ \\
\hline \multirow{2}{*}{ Maniok } & 0,19 & 0,04 & 0,04 \\
& $(0,15)$ & $(0,03)$ & $(0,05)$ \\
Banane & 0,01 & 0,01 & 0,00 \\
& $(0,01)$ & $(0,01)$ & $n c^{1}$ \\
\hline \multirow{2}{*}{ Brache } & 0,18 & 0,09 & 0,07 \\
& $(0,11)$ & $(0,04)$ & $(0,06)$ \\
\hline Gesamtfläche der & 1,59 & 0,88 & 0,42 \\
annuellen & $(0,36)$ & $(0,27)$ & $(0,09)$ \\
Kulturpflanzen & & &
\end{tabular}

Die reichen Familien bauen dreimal mehr Kulturpflanzen an als arme Familien. Die Bracheflächen der reichen Familien sind doppelt so groß wie die der mittelständischen Gruppe.

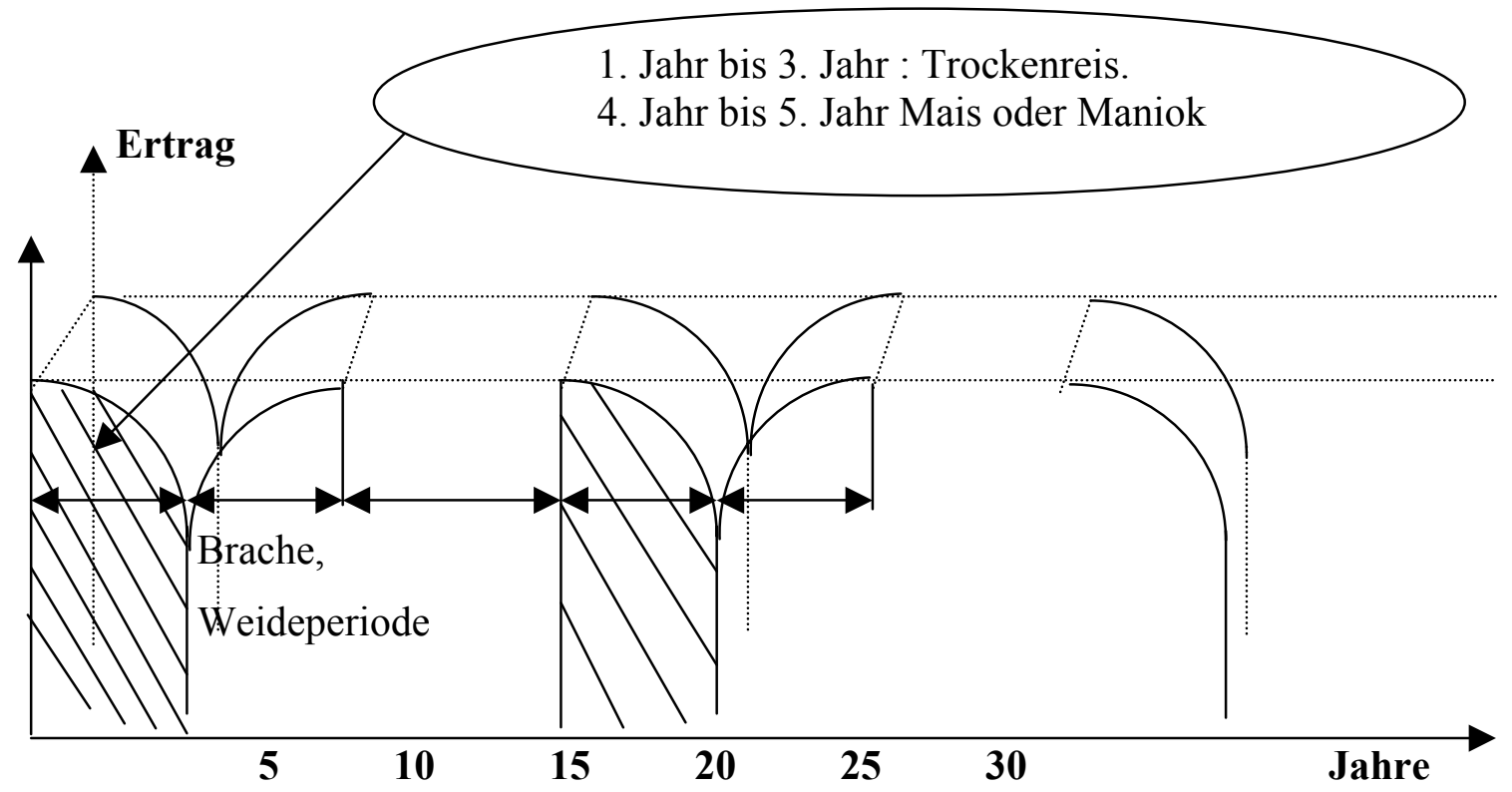

Abb. 13: Natürliche Brache im Untersuchungsgebiet in den letzten 15-30 Jahren 
Die Brachefläche hat einen Anteil von 15\% an der Gesamtfläche. Das bedeutet, dass die Brachezeit verkürzt werden muss, um die Ernährung sicherzustellen

Betrug die Brachezeit vor 80 Jahren noch 10-15 Jahre, liegt sie im Moment bei nur 3-4 Jahren. Hierin liegt wiederum eine Ursache der sehr schnell ablaufenden Bodendegradation.

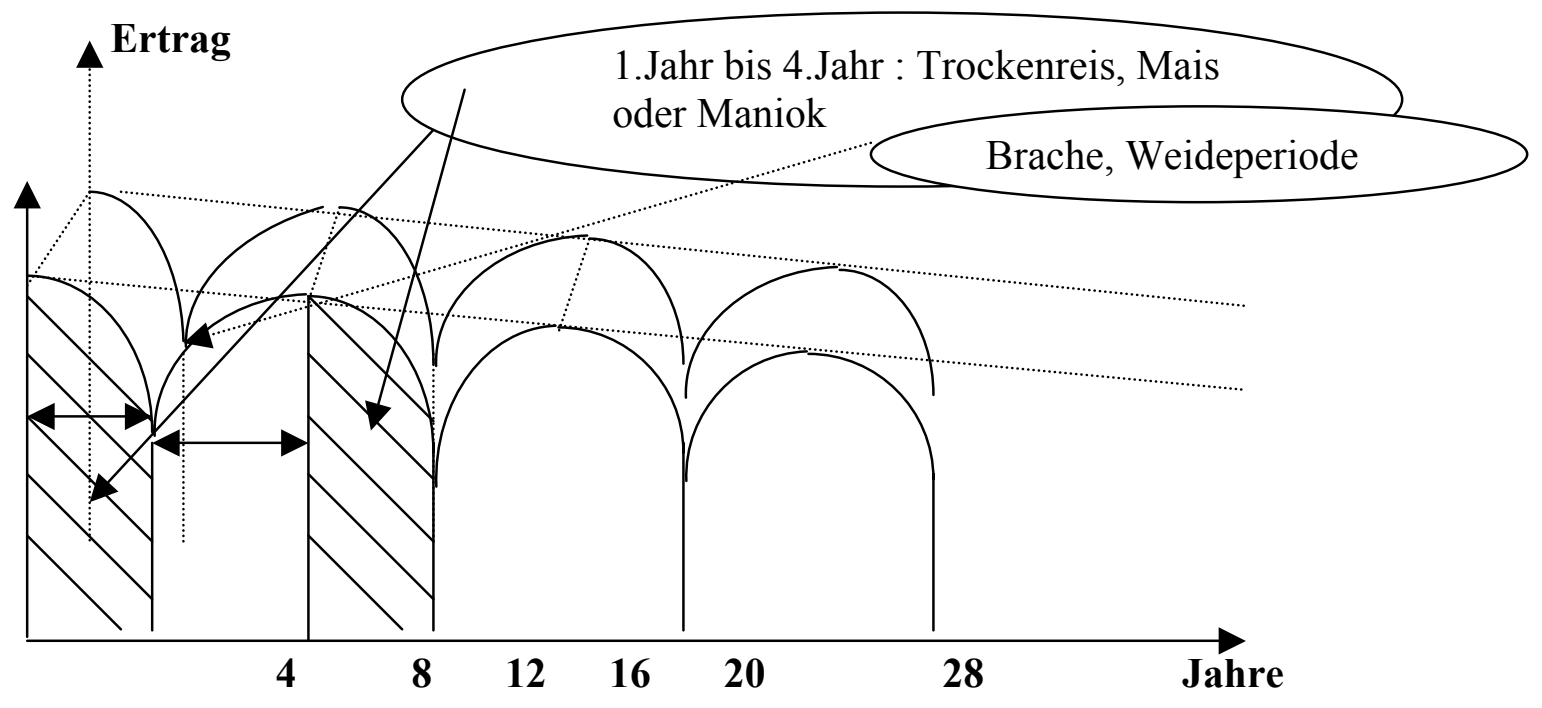

Abb. 14: Natürliche Brache im Untersuchungsgebiet heute

Die typischen Fruchtfolgen sind in den folgenden Schemata 13 \& 14 dargestellt. Außer dem Nassreis ist als Grundnahrungsmittel Mais auch eine wichtige Kulturpflanzenart der Bergfelder. Seit einigen Jahren wird auf den mittelständischen und armen Höfen nur noch wenig Trockenreis angebaut, weil der Ertrag im Trockenreisanbau gesunken ist.

Ca. 70\% der reichen Betriebe haben eine größere Brachefläche als die Betriebe der zweiten Gruppe. 


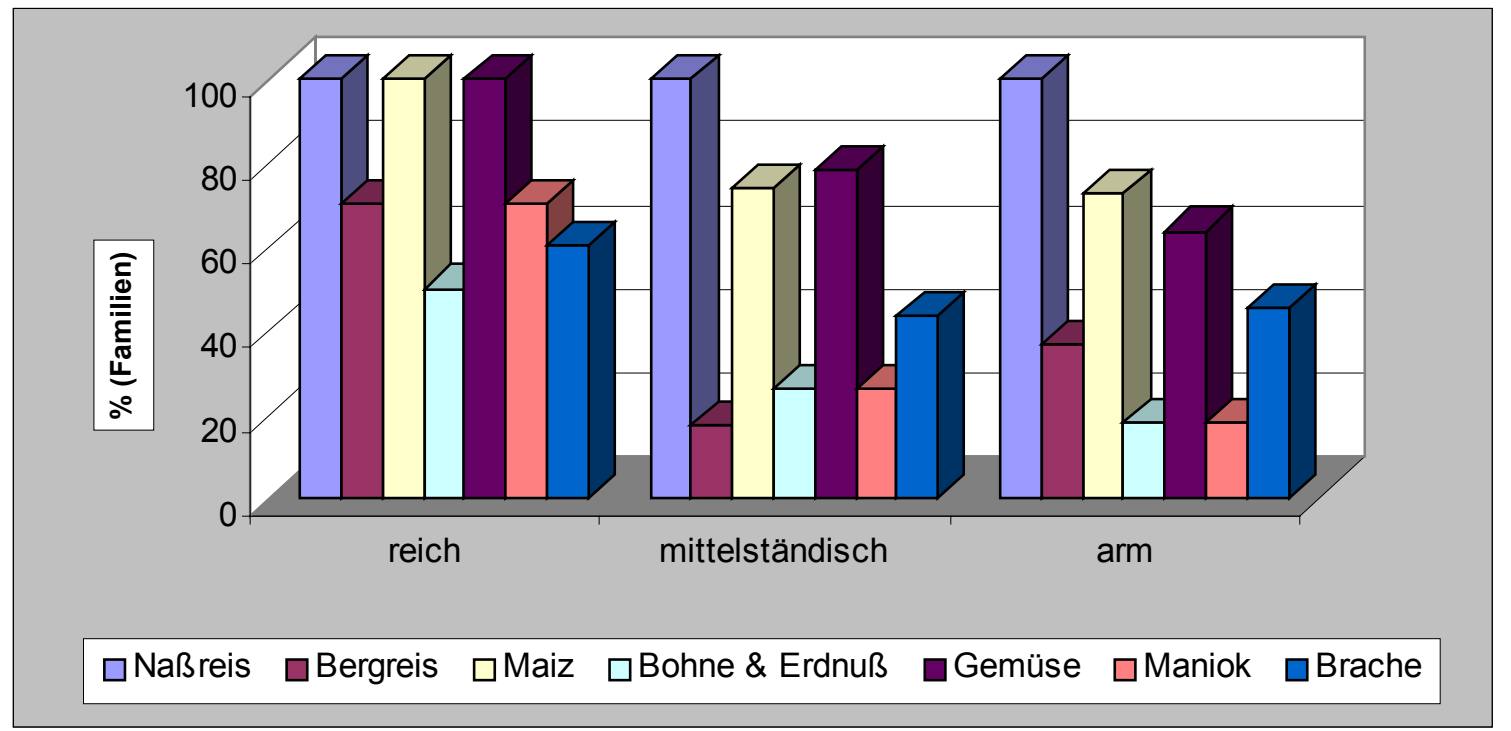

Abb. 15: Angebaute Kulturarten in den verschiedenen Betriebsgruppen in Dia Linh - Ba Be, 2001

Quelle: Eigene Erhebung

Die Obstbaumkulturen spielen eine untergeordnete Rolle in der Untersuchungsregion. Sie tauchen nur in Hausgärten auf. Zuckerrohr sowie Lichi oder Banane werden auf kleinen Parzellen im Hausgarten angebaut und hauptsächlich als „Süßigkeit“ für Kinder verwendet. Die reichen Familien haben durchschnittlich 0,57 ha Hausgarten, in denen die unterschiedlichsten Sorten angebaut werden. Durch den Absatz des Obstes, verfügen die Reichen über eine weitere Einkommensquelle. In den anderen beiden Gruppen fällt der Anbau in Hausgärten kaum ins Gewicht.

Tab. 17: Obst Anbau pro Betrieb in Dia Linh-Ba Be, 2001

\begin{tabular}{lc}
\hline Gruppe Familien & Fläche von Obst (ha) \\
\hline Reich $(\mathrm{n}=11)$ & $0,57(0,23)$ \\
\hline Mittel $(\mathrm{n}=23)$ & $0,09(0,05)$ \\
\hline Arm $(\mathrm{n}=11)$ & $0,11(0,07)$ \\
\hline
\end{tabular}

Note: Der Wert in der Klammer ist das Konfidenzintervall

Kruskal Wallis (Fläche von Obst) Test $\quad$ Chi-Square $=12,136 \quad$ Asymp. Sig $=0,002$

\subsubsection{Jahresarbeitskalender der Familien in Untersuchungsgebiet.}

Der Jahresarbeitskalender einer in der Landwirtschaft und Waldnutzung tätigen Familie in Dia linh wird im folgenden beschrieben. 


\section{Landwirtschaft}

Trockenzeit Februar - April: Nach dem Neujahrsfest, meist Anfang Februar, erkunden die Bauern Flächen für die Brandrodung. Sie roden sowie säubern die Felder und bearbeiten den Boden. Im März wird Winterreis gepflanzt. In dieser Zeit verbringen die Bauern je einen halben Tag auf den Bergfeldern und auf den Nassreisfeldern.

Regenzeit Mai - August: Sobald der erste Regen einsetzt, werden Bergkulturen wie Trockenreis, Mais, Bohnen, Maniok u.a. gepflanzt bzw. gesät. Die Kulturpflege von Winterreis wird durchgeführt. Dies ist die Zeit sehr hoher Arbeitsbelastung. Die Bauern verbringen oft den ganzen Tag auf dem Feld. Nach dem Pflanzen beschäftigen sich die Bauern nur noch mit Kulturpflege, insbesondere dem Unkrautjäten. Die Pflegearbeiten sind sehr zeitaufwendig, da der Wuchs von Unkräutern sehr stark ist. Im Juni wird der Winterreis geerntet. Danach werden die Flächen für das Pflanzen von Sommerreis vorbereitet.

Trockenzeit September - Januar: In diesen Monaten werden verschiedene Bergkulturen geerntet. Die Ernte von Mais, Maniok, und Gemüse erfolgt im September, die von Trockenreis im Oktober und von Sommerreis ab Mitte November. Im Dezember fallen kaum noch landwirtschaftliche Tätigkeiten an. Die Tay bereiten dann das Neujahrsfest vor.

\section{Waldnutzung}

Trockenzeit September - April: Brennholz wird vorwiegend in der trockenen Saison von November bis Januar gesammelt. Es wird eine Brennholzreserve für die Regenzeit angelegt. Die Sammeltätigkeit von Tot- oder Trockenholz wird überwiegend von Frauen durchgeführt. Die Phase landwirtschaftlicher Ruhe von November bis Januar wird für die Gewinnung von Bauholz und Bambusstangen genutzt, Häuser werden gebaut und repariert. Diese Zeit ist auch die wichtigste Jagdsaison. Aber aufgrund der zurückgehenden Waldflächen und Wildtierbestände in den Einzugsbereichen der Siedlungen verliert die Jagd an Bedeutung.

Regenzeit Mai - August: Das Sammeln von Waldprodukten wie Pilze, Bambussprossen, Gemüse u.a. konzentriert sich auf die Regenzeit, die Periode des intensiven Wachstums. Die Frauen gehen im Durchschnitt zweimal pro Woche in den Wald. Ein Teil der Produkte wird direkt vermarktet. Außerdem werden geringe Mengen Brennholz gesammelt. Heilpflanzen werden ganzjährig je nach Bedarf gesammelt. 


\section{Weitere Beschäftigungen}

Handarbeiten, spinnen, weben, stricken und nähen, gehören ganzjährig zu den wichtigen Tätigkeiten der Tay-Frauen. Der Schwerpunkt dieser Arbeiten liegt jedoch im Zeitraum der landwirtschaftlichen Ruhephase. Hauswirtschaftliche Tätigkeiten sind saisonunabhängig.

\begin{tabular}{|c|c|c|c|c|c|c|c|c|c|c|c|c|}
\hline \multirow{3}{*}{ Aktivitäten } & \multicolumn{12}{|c|}{ Saison } \\
\hline & \multicolumn{3}{|c|}{$\begin{array}{l}\text { Trockenzeit } \\
\text { (kühl, trocken) }\end{array}$} & \multicolumn{6}{|c|}{$\begin{array}{c}\text { Regenzeit } \\
\text { (warm, feucht) }\end{array}$} & \multicolumn{3}{|c|}{$\begin{array}{l}\text { Trockenzeit } \\
\text { (Kalt, trocken) }\end{array}$} \\
\hline & Feb. & März & April & Mai & Juni & Juli & Aug. & Sep. & Okt. & Nov. & Dez. & Jan. \\
\hline \multicolumn{13}{|c|}{ Feldbau } \\
\hline \multicolumn{13}{|l|}{ Trockenreis } \\
\hline \multicolumn{13}{|l|}{ Mais } \\
\hline \multicolumn{13}{|l|}{ Bohnen } \\
\hline Maniok & ए & I & & & & & & & & & & \\
\hline \multicolumn{13}{|l|}{ Viehhaltung } \\
\hline \multicolumn{13}{|c|}{ Waldnutzung } \\
\hline \multicolumn{13}{|l|}{ Bauholz } \\
\hline \multicolumn{13}{|l|}{ Bambus } \\
\hline \multicolumn{13}{|l|}{ Brennholz } \\
\hline \multicolumn{13}{|l|}{ Pilze/Gemüse } \\
\hline Hönig & & & & & ए & & & & & & & \\
\hline
\end{tabular}

Abb. 16: Jahresarbeitskalender der Familie in Dialinh, Babe District

\subsubsection{Geschlechtsspezifische Arbeitsteilungen bei den Tay}

\section{Landwirtschaftliche Tätigkeiten}

Die meisten Arbeiten werden von beiden Geschlechtern ausgeführt. Dennoch gibt es geschlechtsspezifische Zuordnungen. $\mathrm{Zu}$ den vorrangig von Frauen ausgeführten Tätigkeiten gehören u.a. Zerkleinern der Erdschollen nach dem Pflügen, Saatgutauswahl, Säen und Pflanzen, insbesondere das Umpflanzen der Reissetzlinge, Säubern, Kulturpflege sowie Lagern und Verarbeiten der Produkte. Bei den für die Frauen typischen zeitaufwendigen Arbeiten wie Säen, Pflanzen oder Kulturpflege helfen normalerweise die Töchter mit. Zu den Hauptaufgaben der Männer gehören das Pflügen, Umgraben und Hacken des Bodens sowie Konstruktion und Instandhaltung der Feldterrassen. Außerdem halten sie Bewässerungskanäle instand und verteilen Dünger auf den Feldern. Zu den weiteren, überwiegend von Männern geleisteten Arbeiten gehören 
die Ernte und der Transport des Getreides vom Feld. Ab einem Alter von 10 Jahren beteiligen sich die Jungen teilweise an den typischen Männerarbeiten.

\section{Gartenarbeit}

Die Arbeit in den kleinen Hausgärten verrichten jüngere und ältere Frauen. Die Männer ernten Früchte von großen Bäumen wie Mangifera sp., Amarindus indica.

\section{Waldarbeiten}

Die wichtigste von Tay-Frauen geleistete Waldarbeit ist das Sammeln von Brennholz, Gemüse und Tierfutter. Die Männerarbeit beschränkt sich im Wesentlichen auf das Baumfällen und den Transport des Holzes. Zu den beliebtesten Tätigkeiten der Männer gehört die Jagd.

\section{Tierhaltung}

Die Frauen sind für Geflügel und Schweine verantwortlich. Die Viehfütterung wird überwiegend von älteren Frauen und Mädchen durchgeführt. Den Tay-Männern obliegt die Großtierhaltung (Büffel und Rinder). Den Weideeintrieb übernehmen ältere Frauen und Männer.

\section{Vermarktung}

Die Tay-Frauen verkaufen Produkte wie Maniok, Früchte und Brennholz direkt auf dem Markt. Die Vermarktung bzw. der Tausch der Tiere gegen andere Waren obliegt den Männern.

\section{Hausarbeiten}

Die jüngeren Frauen übernehmen die Feldarbeiten. Die älteste Frau unterhält tagsüber das Feuer am Herd und übernimmt das Kochen für die Familie. Sie verwaltet die Vorräte und Wertsachen im Haus. Zu weiteren von Frauen und Mädchen geleisteten Arbeiten gehören Wasserholen, Saubermachen, Waschen, Kinderpflege und das Einkaufen für die tägliche Versorgung. Die Männer helfen bei derartigen Arbeiten im Haushalt mit. Die Organisation der Arbeiten obliegt der ältesten Frau.

\section{Andere Tätigkeiten}

Die Tay-Frauen stellen traditionell Matrazen, Tücher, Kissen und Schals als Aussteuer her, und sie sind für die Zucht von Seidenraupen zuständig. Die Mädchen lernen schon im Alter von etwa zehn Jahren das Nähen und Stricken. $\mathrm{Zu}$ den typischen Handarbeiten für Männer gehört das Flechten der Bambusmatten, das Knüpfen von Fischnetzen, die Herstellung verschiedener Haushaltsgeräte und das Bauen von Webrahmen. 


\subsubsection{Bodenqualität und nachhaltige Nutzung}

Der Boden dient dem Menschen u.a. als Produktionsgrundlage für Nahrungs- und Futtermittel und pflanzliche Rohstoffe. Neben der Größe der Anbaufläche ist die Bodenqualität ein wichtiger Faktor, der über die Ernährungsgrundlage und das Einkommen der Familien im Untersuchungsgebiet entscheidet.

Tab. 18: Die Bodenqualität von einzelnen Haushalten, Dia Linh-Ba Be, 2001

\begin{tabular}{|c|c|c|c|c|c|c|c|c|c|}
\hline \multirow[t]{3}{*}{ Bodenfläche (ha) } & \multirow{2}{*}{\multicolumn{2}{|c|}{$\begin{array}{c}\begin{array}{c}\text { reich } \\
(\mathrm{n}=11)\end{array} \\
1\end{array}$}} & \multirow{2}{*}{\multicolumn{2}{|c|}{$\begin{array}{c}\begin{array}{c}\text { mittelmäßig } \\
(\mathrm{n}=23)\end{array} \\
2\end{array}$}} & \multirow{2}{*}{\multicolumn{2}{|c|}{$\begin{array}{c}\begin{array}{c}\text { arm } \\
(\mathrm{n}=11)\end{array} \\
3\end{array}$}} & \multicolumn{3}{|c|}{$\begin{array}{l}\text { Signifikanter } \\
\text { Unterschied }\end{array}$} \\
\hline & & & & & & & \multirow{2}{*}{$1-2^{1}$} & \multirow{2}{*}{$1-3^{2}$} & \multirow{2}{*}{$2-3^{3}$} \\
\hline & ha & $\%$ & ha & $\%$ & ha & $\%$ & & & \\
\hline $\begin{array}{l}\text { ebene } \\
\text { Terrassenfläche } \\
\text { (Nassreisanbau) }\end{array}$ & $\begin{array}{r}0,60 \\
(0,24)\end{array}$ & 25 & $\begin{array}{r}0,46 \\
(0,11)\end{array}$ & 45 & $\begin{array}{r}0,17 \\
(0,05)\end{array}$ & 30 & - & $* * *$ & $* * *$ \\
\hline Bergfelder & $\begin{array}{r}1,76 \\
(0,95)\end{array}$ & 74,6 & $\begin{array}{r}0,53 \\
(0,25)\end{array}$ & 53,5 & $\begin{array}{r}0,37 \\
(0,20)\end{array}$ & 68,8 & $* *$ & $* *$ & - \\
\hline
\end{tabular}

Note: Werte in Klammern (Konfidenzintervall) des geschätzten Mittelwertes $(\alpha=0,1)$

1,2,3 Wahrscheinlichkeitsdichtefunktion (Vergleich von zwei unabhängigen Stichproben) zwischen Familien nach MANN-WHITNEY test(S.270): *** $=99 \% ; * *=95 \% ; *=90 \%$.

Das Dorf Dia Linh befindet sich im Hochland. Der Nassreis wird deshalb auf ebenen Flächen an schmalen Bachufern oder in engen Tallagen angebaut. Die gesamte ebene Bodenfläche des Dorfes ist sehr gering, so dass reiche oder arme Familie nur eine kleine Fläche zum Reisanbau besitzen. Nach Tab.18 besitzen reiche Familien im Durchschnitt 0,6 ha Nassreisflächen, arme Familien dagegen nur 0,17 ha.

Der Flächenanteil der Bergfelder der reichen Familien beträgt 74,6\% und der von mittelständischen Familien 53,5\% der gesamten Fläche des Dorfes. Sie weisen zumeist eine Hangneigung von $20^{\circ}$ bis $40^{\circ}$ auf. D. h. sie sind sehr gefährdet durch Erosion vor allem durch hohe Niederschlagsmengen und Monokultur. Die Erhaltung der Bodenfruchtbarkeit ist dadurch besonders schwierig, weil die Brachperiode des traditionellen Wanderfeldbaues aufgrund des erhöhten Bevölkerungsdrucks immer verkürzt wird und der Boden kann sich durch ständige Nutzung nicht erholen. Bei Befragung beantworteten die meisten Landwirte übereinstimmend, dass die Bodenfruchtbarkeit von mehr als 70\% ihres Landes bereits abgenommen hat. Nach eigener Schätzung könnte der Anteil der degradierten Fläche noch höher liegen. Die Wiederherstellung der Bodenfruchtbarkeit auf der bergigen Landschaft Dia Linh bzw. im Bereich der vorliegenden Studie wäre deshalb eine außerordentlich schwierige Aufgabe. 
Nun stellt sich die Frage, welche Art von Kultur könnte in einem Jahr zu hoher Bodenerosion im Bergland während der Niederschlagsperiode führen? Die Antwort auf diese Frage ist zu einem aus dem Untersuchungsergebnis der unten liegenden Tabelle zu finden. Zum anderen ergibt sie sich aus Einschätzungen der ortsansässigen Bauern selbst. Demnach verursacht Hochlandreis, Erdnuß und Mais die stärkste Rillenerosion. Dagegen führt Maniok, nach Einschätzung von Bauern, zu niedriger Bodenerosion, denn Maniok wächst die ganze Zeit und lässt den Bauern 2 Jahre ernten. Der Boden wird während dieser Zeit beschattet und weitgehend geschützt.

Tab.19 : Bodenerosion nach Art der Kulturpflanzen (\% Fläche)

\begin{tabular}{lrrr}
\hline Rillenerosion & $<5 \mathrm{~cm}$ & $5-10 \mathrm{~cm}$ & $>10 \mathrm{~cm}$ \\
\hline Trockenreis & 0,43 & 2,23 & 97,34 \\
\hline Mais & 37,61 & 43,51 & 18,88 \\
\hline Bohne & 13,51 & 54,05 & 32,43 \\
\hline Cassava & 42,21 & 43,18 & 14,61 \\
\hline Brache & 2 & 0 & 0 \\
\hline Sekundärwald & 0 & 0 & 0 \\
\hline Quelle: Eigene Erhebung & & &
\end{tabular}

Die o.g. Übersicht lässt erkennen, dass die Erosionsintensität von der Art der Kulturpflanzen abhängig ist. Die überwiegende Mehrheit der Landbevölkerung erkennt dies leider nicht. Sie besitzt keinerlei fundierten Informationen über Ursachen, Gefahren und Folgen der Bodenerosion. Nur 22,8\% der befragten Bauern erkennen, dass Bodenerosion ein schwerwiegendes Problem ist, $32 \%$ wissen nichts darüber oder gaben keine Antwort. 54,8\% haben gewusst, dass starker Niederschlag der Grund für Bodenerosion ist.

Nur 4,5\% von Bauern bauen Hecken als Maßnahme zum Schutz vor Bodenerosion. Dagegen geben 62,3\% Bauer an, dass sie überhaupt nicht von Bodenerosionsschutzmaßnahmen wissen. Sie sind daran gewöhnt, die Brachperiode als Zeitraum für die Rehabilitation der Bodenfruchtbarkeit im traditionellen Wanderfeldbau anzusehen. Für die Wissenschaftler ist deshalb die Suche nach neuen Verfahren bzw. Techniken zur Verhinderung von Bodenerosion und nach angepassten Anbausystemen zur Unterstützung einer nachhaltigen Flächennutzung auf bergigen Landschaften eine Herausforderung. 
Tab.20: Meinung der Bauern zu Fragen des Bodenerosionsschutzes

\begin{tabular}{|l|c|}
\hline Frage nach & Anteil der Bauern antworten mit ja [\%] \\
\hline der Bodenerosion: & 22,8 \\
- ist schwerwiegend & 35,2 \\
- hat keinen Einfluss & 32,0 \\
- Bodenerosion ist unbekannt & 10,0 \\
- keine Angaben & \\
\hline den Gründen für Bodenerosion & 54,8 \\
- Niederschlag & 37,6 \\
- große Hangneigung & \\
- $\quad$ falsche Bodenbearbeitung, fehlende & 28,5 \\
$\quad$ Bodenbedeckung & 34,1 \\
- keine Angaben & \\
\hline genutzten & \\
Bodenerosionsschutzmaßnahmen & 6,5 \\
- Bodenbedeckung durch Pflanzenreste & 4,5 \\
- Anlegen von Heckenreihen & 32,6 \\
- Terrassierung & 9,9 \\
- Terrassierung mit Grenzmauer & 4,5 \\
- Benutzen mehrerer Maßnahmen & 62,3 \\
- keine Anwendung von Schutzmaßnahmen & \\
\hline Quelle: Eigene Erhebung &
\end{tabular}

Die Länder, die noch keine positiven Reaktionen auf wachsende Bodenerosion zeigen, lassen politischen Willen mangels Aufklärung vermissen. Im Laufe der vergangenen Generation haben viele Länder Nahrungsmitteldefizite erfahren, aber nur wenige haben diese Einbußen mit der Zerstörung ihre Böden durch Erosion in Verbindung gebracht. In vielen vorwiegend ländlichen Gesellschaften, wo die meisten Bewohner Analphabeten sind und am Rande des Existenzminimums leben, hat das mangelnde öffentliche Interesse am Bodenschutz andere Ursachen. In vielen Dörfern der Dritten Welt können die Bauern wenig Interesse für die Zukunft aufbringen, wenn schon ihr gegenwärtiges Überleben nicht gesichert ist (BROWN \& WOLF, 1985).

Es zeigt sich daran, wie dringend notwendig eine Beratung und Zusammenarbeit mit den Bauern zu Fragen des Erosionsschutzes ist, was auch ein Ziel dieser Arbeit darstellt. 


\subsubsection{Forstwirtschaft}

Nach PRIMENTEL (1989) ist Wald eine wichtige Naturressource für die künftige Entwicklung. Aufgrund seiner herausragenden Bedeutung sollte seine gegenwärtige Nutzung, bisherige Schutzmaßnahmen sowie seine Funktionsveränderungen untersucht werden.

\subsubsection{Durchschnittliche Waldfläche jeder Familie in Dia Linh}

Tabelle 21 zeigt die durchschnittliche Waldfläche, die jeder Familie in Dia Linh für 15-20 Jahre durch den Staat zur Nutzung überlassen wurde (,green certificate book ${ }^{6}$ ).

Tab.21: Durchschnittliche Waldfläche pro Haushalt, Dia Linh 2001

\begin{tabular}{|c|c|c|c|}
\hline Waldfläche & $\begin{array}{l}\text { Reich } \\
(\mathrm{n}=11)\end{array}$ & $\begin{array}{l}\text { Mittel } \\
(n=23)\end{array}$ & $\begin{array}{c}\text { Arm } \\
(\mathrm{n}=11)\end{array}$ \\
\hline Waldfläche & $\begin{array}{r}2,89 \\
(0,83)\end{array}$ & $\begin{array}{r}1,66 \\
(0,70)\end{array}$ & $\begin{array}{r}1,20 \\
(0,73)\end{array}$ \\
\hline \multicolumn{4}{|l|}{ Davon Anteil \%: } \\
\hline - Sekundärwald & 24,56 & 27,62 & 18,83 \\
\hline - Neu Anbau & 75,44 & 72,38 & 81,17 \\
\hline
\end{tabular}

Note: Der Wert in der Klammer entspricht dem Konfidenzintervall der Mittelwertschätzung $(\alpha=0.1)$

Kruskal Wallis (Waldfläche) Test Chi-Square $=7,18 \quad$ Asymp. Sig $=0,028$

Außerdem gibt es noch Waldflächen, die unter direktem Management der Regierung stehen. Das meiste dieser Waldflächen stellt der Primärwald im Nationalpark Babe dar. Obwohl es gesetzlich verboten ist, nutzen die Bauern diesen Wald im Nationalpark als Ressource für Brennholz, Pilze, Bambussprossen und Pflanzenheilkunde.

Aus Tab.21 sieht man, dass reiche Familien über mehr Waldfläche verfügen als arme Familien. Neuer Anbauwald stellt hierbei mit über $3 / 4$ den überwiegenden Anteil dar. Dies sind in der Regel Flächen, die früher zur Brandrodung genutzt wurden. Zum Teil ist die Bodenqualität noch sehr schlecht und es können nur Wildkräuter angebaut werden oder einzelne Sträucher und Alang Alang tritt auf.

Daher stellt sich die Frage, wie die Bauern den Wald nutzen?

\subsubsection{Waldnutzung durch die Bauern.}

In Anlehnung an die Anweisung zur Waldinventur in Vietnam wurden Formen der Landnutzung und deren Flächendeckung in den untersuchten Dörfern bestimmt. Die wichtigsten Kategorien in der Landwirtschaft sind bewässerte Kulturen, Regenfeldbau auf 
meist steilen Berghängen und Tierhaltung. In der Forstwirtschaft unterscheidet man die Kategorien v.a. nach der Nutzungsintensität von Naturwald.

Tab.22: Vorrangige Waldnutzung durch Haushalte, Dia Linh 2001

\begin{tabular}{lrrrr}
\hline Vorrangig & $\begin{array}{c}\text { Reich } \\
(\mathrm{n}=11)\end{array}$ & $\begin{array}{c}\text { Mittel } \\
(\mathrm{n}=23)\end{array}$ & \multicolumn{2}{c}{$\begin{array}{c}\text { Arm } \\
(\mathrm{n}=11)\end{array}$} \\
\hline 1. Brennholz & 72,7 & 95,7 & 90,9 \\
\hline 2. Nahrung & 45,5 & 87,0 & 100 \\
\hline 3. Medizin & 54,6 & 65,2 & 45,5 \\
\hline 4. Wasser & 18,2 & 43,5 & 27,3 \\
\hline 5. Holz & 9,1 & 8,7 & 18,2 \\
\hline 6. Weide Fläche & 63,6 & 73,9 & 72,7 \\
\hline 7. Forst/Obst & 72,7 & 78,3 & 81,8 \\
\hline 8. Keine Nutzung & 18,2 & 8,7 & 0,0 \\
\hline
\end{tabular}

Die Nutzung des Waldes im Untersuchungsgebiet lässt sich in folgende Kategorie einordnen:

\section{Gering genutzter Naturwald:}

Vorratsreiche Wälder mit Starkholzanteil wirtschaftlich wertvoller Holzarten. Bisher wurden lediglich einzelne Starkhölzer genutzt. Hauptbaumarten sind u.a. Bischofia trifolia, Chukrasia tabula-ris,Cinnamomum iners, Cinnamomum tonkinensis, Garcinia fragnaeoides, Pentacetonkinensis, Diospyros bangdiensis.

In Dia linh zählen ca. 323,5 ha Wald zu dieser Kategorie. Sie befinden sich in schwer zugänglichen Lagen und dienen vor allem dem Wasserschutz. Für die Dorfbewohner gelten strenge Nutzungsbeschränkungen. Holzeinschlag ist verboten. Aber es konnte diese Waldformation nicht nachgewiesen werden. Holzexploitation und Waldbrände in den letzten Jahrzehnten sind hierfür die Gründe.

\section{Mäßig und stark genutzter Naturwald:}

Waldflächen mit Vorrat an Sägeholz. Wertvolle Stämme wurden meist schon abgeholzt.

Die Nutzungsmöglichkeit ist daher beschränkt. Typische Vertreter sind Keteleeria davidiana, Quercus ancustissima, Quercus chevalieri, Schima superba, Podocarpus, impricatus, Pinus mercusiana. Dieser Waldzustandstyp ist durch unkontrollierte Nutzung aus der zuerst genannten Kategorie hervorgegangen.

Dieser Waldzustandstyp nimmt in diesen Dörfer den größten Anteil der Waldfläche ein. Nach Aussagen der Dorfbewohner resultieren derartige Bestände aus dem kommerziellen Holzeinschlag in den 70er und 80er Jahren. 
Jede Arbeitskraft hatte entsprechend staatlichen Bestimmungen 30 Holz- bzw. Bambusstangen pro Jahr an die Forstverwaltung zu liefern. Waldbauliche Maßnahmen zur Reproduktion gab es jedoch nicht.

\section{Sekundärwald:}

Vorwälder und vorwaldähnliche Bestockungen sowie künstlich begründete Bestockungen bis zum Stangenstadium (durchschnittliche Durchmesser $<20 \mathrm{~cm}$ ). Bäume von Sägeholzdimensionen sind nicht vorhanden. Hier kommen vorwiegend lichtbedürftige Pionier- und schnellwachsende Baumarten vor. Diese Baumarten sind u.a. Bischofia trifoliata, Litsea cubeba, Helicteres angustifolia, Pterospermum rucatolobatum,Sterculia nobilis, Canarium album, Castanapsis sinensis, Eugenia wightiana,Shima superba, Styrax tonkinensis, Chromolaene Odotata, Imperata Cylindrica.

Mit 876,47 ha ist die Fläche dieses Waldzustandstyps etwa so groß wie die des mäßig und stark genutzten Naturwaldes. Teilweise entsteht dieser Vegetationstyp auf Brachflächen nach dem Brandrodungsfeldbau, teilweise durch starke Waldexploitation.

\section{Bambuswald:}

In Dia Linh wachsen über 14 Bambusarten . Die vorkommenden Arten sind Bambusa arundinacea, Bambusa multiplex, Bambusa ventricosa, Gigantochloa scribneriana, Calamus rudentum, Calamus tennis, Dentrocalamus patellaris, Dentrocalamus latiflorus u.a.

Bambus wächst im Dorfwald sowie im Dorf bzw. auf landwirtschaftlich genutzten Flächen. Mit Ausnahme von Indosasa sinica, Pleioblastus sp. und teilweise Cephalostachyum pergracile ist er ausnahmslos kultiviert. Im Wald ist er auf siedlungsnahe Ränder beschränkt. Aus der Pflanzung von Bambus ergeben sich Nutzungsrechte. Vorrangig wird Bambus als Stützkonstruktionen für kleinere Pfahlbauten und für den Schlafzimmerboden der Häuser, Webstühle sowie Konstruktionen zum Räuchern über den offenen Feuerstellen im Haus verwendet. Teppiche, Körbe und andere Aufbewahrungbehältnisse, Fischfallen, Stühle und Tische werden als Flechtarbeiten aus Bambus hergestellt. Cephalostachyum pergracile dient als Kochgefäß für Reis. Aus Schizostachyum funghonii werden Raketen für das Wasserfest hergestellt, aus Dendrocalamus hamiltonii können Stricknadeln gefertigt werden. Nicht zu vergessen sind die Herstellung von Eßstäbchen aus einigen Arten sowie die Verwendung von Bambus als Opfergabe an heiligen Bäumen.

Der Anteil von Bambuswald ist 90 ha. Die Bambusarten beigemischt sind vor allem Lichtbaumarten, die nach der Exploitation des Naturwaldes verblieben sind. Der 
Bambuswald liefert Baumaterial für den Hausbau und für Haushaltsgeräte sowie Bambussprossen als Gemüse.

\section{Sonstige Nicht-Holzprodukte}

Die Tay sammeln eine Vielzahl von Nicht-Holzprodukten im Wald. Diese Sammelaktivitäten werden meist neben anderen Tätigkeiten ausgeführt. Ihre ökonomische Bedeutung in einer Selbstversorgungsgesellschaft ist nicht zu unterschätzen. Auf diesem Feld hat sich eine Fülle an indigenem Wissen und spezieller Nutzungstechnologie herausgebildet. Es wird fast ausschließlich für den Eigenbedarf gesammelt. Viele der früher im Wald gesammelten Produkte werden heute in den Hausgärten angebaut. Einige identifizierte Arten und ihre Nutzung werden unten, ohne den Anspruch auf Vollständigkeit, angeführt.

Saururaceae: Saurura chinensis (Lour.), Pteridiaceae: Pteridium aquilium var. latiusculum (L.): gesamte Pflanze als Gemüse genutzt.

Caesalpiniaceae: Bauhinia variagata (L.): Blüten werden als Gemüse genutzt.

Fagaceae: Castanopsis hystrix A. DC.: eßbare Früchte C. indica (Toxb.) DC.: eßbare Früchte

C. mekongensis A. Camus.; Guttiferae: Garcinia xanthochymus : eßbare Früchte

Moraceae: Ficus auriculata Lour.: eßbare Früchte, junge Blätter werden als Gemüse genutzt.

F. callosa Willd.; F. glomerata Roxb und F. lacor Buch.-Ham: junge Blätter werden als Gemüse genutzt.

Burseraceae: Canarium album (L.): eßbare Früchte C. strictum Roxb.: eßbare Früchte

Juglandaceae: Pterocarya tonkinensis Dode: Blätter als Fischgift verwendet, Herstellung von Schießpulver aus Holzasche, Brennmaterial bei religiösen Zeremonien.

Solanaceae: Solanum nigrum (L.): gesamte Pflanze als Gemüse verwendet.

Zingiberaceae: Alpinia galanga (L.) Willd.: Früchte zur Arzneimittelherstellung verwendet.

Palmae: Calamus spp.: Rattan zur Möbelherstellung

Gramineae: Imperata cylindrica (L.) Beauv. var. major (Nees) C. E. Hubb.: Genutzt zum Dachdecken.

Aufforstung: Nach Angabe des Dorfes Dia Linh wurden insgesamt 315 ha Aufforstungen mit schnellwachsenden Baumarten wie Melia azedarach, Tectonagrandis, Eucalytus camaldulensis und Eucalyptus tereticornis durchgeführt. 


\subsubsection{Einkommen aus Wald}

Nicht nur die Landwirtschaft, sondern auch die Waldnutzung spielt eine wichtige Rolle für die Generierung von Einkommen der Tay-Bauern.

Tab.23: Durchschnittliche Einkommen aus Wald, Dia Linh 2001

\begin{tabular}{lllll}
\hline Gruppe & \multicolumn{1}{c}{ Mittelwert Einkommen (in 1000 VND) } \\
\hline Reich $(\mathrm{n}=11)$ & & $1743,5(245,7)$ \\
\hline Mittel $(\mathrm{n}=23)$ & & & $1328,6(177,3)$ \\
\hline Arm $(\mathrm{n}=11)$ & & $1098,2(182,0)$ \\
\hline $\begin{array}{l}\text { Quelle: Eigene } \\
\text { Mittelwertsschätzung }(\alpha=0.1) \\
\text { Kruskal Wallis Test (Forest income) }\end{array}$ & Chi-Square $=1,264 \quad$ Asymp. Sig $=0,37$
\end{tabular}

Tab. 23 zeigt die Einkommen der Familien unterschiedlichen Lebensstandards aus der Waldnutzung. Bei den reichen Familien trägt dieses Einkommen 8,7\%, bei den mittelständischen Familien 12,1\% und bei den armen Familien 18,2\% zum Gesamteinkommen bei. Arme Familien sind also auf Einkommen aus der Waldnutzung stärker angewiesen. Insgesamt zeigt sich aber, dass das Haupteinkommen der Tay-Bauern aus der Landwirtschaft kommt. Deswegen ist nachhaltige Landnutzung in dieser Untersuchung besonders wichtig.

\subsection{Einkommen}

Die Landwirtschaft steht heute im Mittelpunkt eines zunehmenden Konfliktes zwischen Ökonomie und Ökologie. Sinkende Erzeugerpreise zwingen einerseits zu einer hohen Bewirtschaftungsintensität mit möglichst wirtschaftlichem Einsatz der Produktionsmittel, während anderseits die Forderung einer zunehmend für Umweltfragen sensibilisierten Gesellschaft nach einer umweltschonenderen Wirtschaftsweise besteht.

Die Einkommensquellen der verschiedenen wirtschaftlichen Aktivitäten der Haushalte in Dia Linh sind in folg. Tab. 24 dargestellt.

Landwirtschaft: Fast $90 \%$ des Familieneinkommens werden in der Landwirtschaft erzielt. Reis und Mais sind die wichtigsten Verkaufsprodukte. Unmittelbar nach der Ernte werden etwa 3-5\% verkauft. Der Erlös dient dem Erwerb von Gebrauchsgütern. Der Anbau von Gemüse spielt eine untergeordnete Rolle.

Gartenwirtschaft: Wichtige Produkte sind Bananen, Mangos, Grapefruit, Orangen, Lichi (lychee), Longen u.a. Sie werden im Haushalt verwendet. Der Verkauf von Früchten ist kaum einkommenswirksam. 
Tab. 24 :Tatsächliche Einkommen von Familien in Dia Linh 2001(1000VND)

\begin{tabular}{lrrrrrr}
\hline \multirow{2}{*}{ Kennzeichnung } & \multicolumn{1}{c}{$\begin{array}{c}\text { Reich } \\
(\mathrm{n}=11)\end{array}$} & $\begin{array}{c}\text { Mittel } \\
(\mathrm{n}=23)\end{array}$ & \multicolumn{2}{c}{$\begin{array}{c}\text { Arm } \\
(\mathrm{n}=11)\end{array}$} & \multicolumn{3}{c}{$\begin{array}{c}\text { signifikanter } \\
\text { Unterschied }\end{array}$} \\
\cline { 2 - 8 } & \multicolumn{1}{c}{1} & \multicolumn{1}{c}{2} & \multicolumn{1}{c}{3} & $1-2^{1}$ & \multicolumn{1}{c}{$1-3^{2}$} & $2-3^{3}$ \\
\hline Einkommen vom Bauernhof & $\mathbf{1 7 3 5 4 , 2}$ & $\mathbf{9 2 3 4 , 6}$ & $\mathbf{4 9 4 6 , 8}$ & $* * *$ & $* * *$ & $* *$ \\
\hline - \% von Einkommen gesamt & 86,60 & 84,04 & 82,04 & & & - \\
\hline - Einkommen/ ha Boden & 3299,3 & 3484,8 & 2843,0 & - & - & $*$ \\
\hline Nicht landwirtschaftliches & $\mathbf{2 6 8 7 , 5}$ & $\mathbf{1 7 5 4 , 4}$ & $\mathbf{1 0 8 2 , 7}$ & $* *$ & $* * *$ & $*$ \\
Einkommen & 13,40 & 15,06 & 17,96 & & & \\
\hline - \% von Einkommen gesamt & $\mathbf{2 0 0 4 1 , 7}$ & $\mathbf{1 0 9 8 9 , 0}$ & $\mathbf{6 0 2 9 , 5}$ & $* * *$ & $* * *$ & $* *$ \\
\hline Familieneinkommen /a & $(1325,4)$ & $(895,3)$ & $(577,8)$ & & & \\
\hline - Einkommen/ Person/a & 2791,3 & 1859,4 & 1144,1 & $* *$ & $* * *$ & $*$ \\
\hline - Einkommen/ Arbeitkraft & 3497,7 & 2383,7 & 1530,3 & $* * *$ & $* * *$ & $* *$ \\
\hline
\end{tabular}

Note: Der Wert in der Klammer entspricht dem Konfidenzintervall der Mittelwertsschätzung $(\alpha=0.1)$

1,2,3 Die Wahrscheinlichkeit, daß keine signifikanter Unterschied zwischen den Klassen besteht beträgt nach dem MANN-WHITNEY test: ***=99\%; **=95\%; $*=90 \%$.

4) $15000 \mathrm{VND}=1$ US\$

Tierhaltung: Die Tierhaltung leistet einen großen Beitrag zur Versorgung der Familien mit Nahrungsmitteln. Schweine und Geflügel sind eine wichtige Bargeldquelle der Tay. Bis zu 80\% der Schweine und 50\% des Geflügels werden verkauft. Der Anteil der Erlöse durch die Tierhaltung erreicht ein Viertel des gesamten Einkommens.

Fischzucht: Fischwirtschaft ist eine neue Komponente in den Betriebssystemen und trägt bisher bei ca. zwei Drittel der Familien geringfügig zum Einkommen bei.

Waldnutzung: Waldprodukte sind eine wichtige Einkommensquelle der Tay. Die Hauptprodukte sind Bambussprossen, Pilze, Nüsse, Gemüse, Heilpflanzen und Honig. Sie sind hauptsächlich für den Eigenverbrauch bestimmt. Einkünfte aus dem Verkauf sind wegen der niedrigen Preise relativ gering. Durchschnittlich sammelt eine Tay-Familie ca. $15 \mathrm{~kg}$ Brennholz pro Tag bzw. $5.475 \mathrm{~kg}$ pro Jahr. Obwohl Holz überwiegend dem Eigenbedarf dient, sind in Monaten der Nahrungsmittelknappheit insbesondere arme Familien auf den täglichen Verkauf von Brennholz angewiesen. Das Brennholz macht dann den größten Anteil am Einkommen durch die Waldnutzung aus.

Tab. 24 zeigt, dass über $80 \%$ der Einkommen aus der Landwirtschaft generiert werden. Die Landwirtschaft spielt daher eine wichtige Rolle im Leben sowie im Einkommen von der Tay- Gruppe im Untersuchungsgebiet. 
Nach HeMmMER 1999 \& FAO 2001 wird die Armutsgrenze bei einem Einkommen von 1 US\$/Tag/ Person angesetzt, d.h. dass die gesamte Tay Minderheit im Untersuchungsgebiet unabhängig davon ob reich oder arm klassifiziert (s. Tab. 24) als arm einzustufen ist.

\subsection{Schlussfolgerung}

Die sozioökonomische Auswertung der Tay-Haushalte im Untersuchungsgebiet ergibt zusammengefasst folgende Ergebnisse:

1- Die typischen Nutzpflanzen der Tay-Bevölkerungsgruppe sind Monokulturen von Mais, Maniok, Nassreis oder Trockenreis. Die Erträge sind allerdings nicht stabil.

2- Der Umfang der Haushalte ist vergleichsweise sehr klein, Pro Familie gibt es durchschnittlich 5-6 Personen, mit jeweils 2-3 Arbeitkräften. Die Bewirtschaftungsfläche pro Haushalt ist klein, wobei sie 0,53ha bei armen Familien und 2,3 ha bei reichen Familien beträgt.

3- Das Leben der Tay-Bevölkerung in Bergland trägt sich hauptsächlich durch die Nutzung natürlicher Ressourcen, besonders aus Boden, Wasser und Wald. Das Einkommen aus der Landwirtschaft beträgt durchschnittlich $80 \%$ während über $15 \%$ des Einkommen aus der Waldproduktion generiert werden. Sowohl arm als auch reich eingestufte Familien im Untersuchungsgebiet haben ein Einkommen von weniger als $1 \mathrm{US} \$$ /Tag/Person und liegen damit unterhalb der nach FAO definierten und international anerkannten Armutsgrenze. Sogenannte reiche Familien haben ein jährliches Einkommen von 186 US\$/ Person.

4- Die Bauern besitzen wenig oder keine Informationen über die Ursachen, Gefahren und Folgen der Bodendegradation.

5- Die Übernutzung der natürlichen Ressourcen, bedingt u.a. durch die zu kurze Brachezeit, hat seine Ursachen in einem übermäßigen Bevölkerungswachstum. Dieses führt zu einem Nahrungsmittelmangel und folglich zur Waldrodung für den Anbau von Kulturpflanzen, das ökologische Gleichgewicht wird dadurch gestört.

6- Die traditionelle Landnutzung in der Bergregion mit Brandrodung kann angesichts eines anhaltend übermäßig hohen Bevölkerungsdrucks nicht fortgeführt werden.

7- Die genannten sozioökonomischen Faktoren lassen eine Verschiebung in Richtung nachhaltiger Flächennutzung wie angepasster Anbausysteme dringend notwendig erscheinen. Es müssen dringlich Maßnahmen angewendet werden, um dieser Probleme zu überwinden. 


\section{ERGEBNIS DER FELDUNTERSUCHUNG UND IHRE BEWERTUNG}

\subsection{Beschreibung der Bodenprofile}

Für die Bodenansprache im Gelände und zur Entnahme der Bodenproben wurden Profilgruben bis zu 1,5 m Tiefe an den 3 untersuchten Standorten ausgehoben. Die Profilbeschreibung im Gelände erfolgte nach der Anleitung zur Beschreibung der Bodenprofile der FAO UNESCO- ISRIC (1989) und in Anlehnung an die Kartieranleitung der A.G BoDENKUNDE (1982) für Bodenart, organische Substanz, Bodengefüge, effektive Lagerungsdichte, und Durchwurzelung. Die Farbansprache erfolgte anhand der MUNSELL- FARBTAFEL (1967) sowohl an feldfeuchten als auch an luftgetrockneten Proben.

\section{Probefläche Nr. 1 (Agroforstsystem mit Mulch)}

Geographische Lage: Ca. 6 km westlich von Dia Linh Gemeindezentrum

Höhe ü NN: $370 \mathrm{~m}$

Geologie: Kieseliger Schiefer, toniger Kalkstein, tuffig-sandiger Schluffstein

Relief: konvexer Mittelhang, $22^{\circ}$ Hangneigung, Exposition SW

Klima: Jahresniederschlag $1500 \mathrm{~mm}$ (Regenzeit von April bis September), durchschnittliche Temperatur im Sommer $28^{\circ} \mathrm{C}\left(\operatorname{Max} .40^{\circ}\right.$, Min. $\left.12^{\circ}\right)$, $15,5^{\circ}$ im Winter (Max. $25^{\circ}$, Min. $4^{\circ}$ ), Luftfeuchtigkeit bei $85 \%$

Vegetation/Nutzung: Seit 3 Jahren neues Landnutzungssystem: Mischkultur aus Tephrocia candida - Heckenreihen und verschiedenen Obst- und Feldkulturen in den Zwischenreihen, vorher Nutzung der Fläche für den Anbau von grünem Tee Hydrologie: grundwasserfern Bodentyp (FAO): Geric Ferralsol (FRg) 


\section{Beschreibung des Profils Nr.1:}

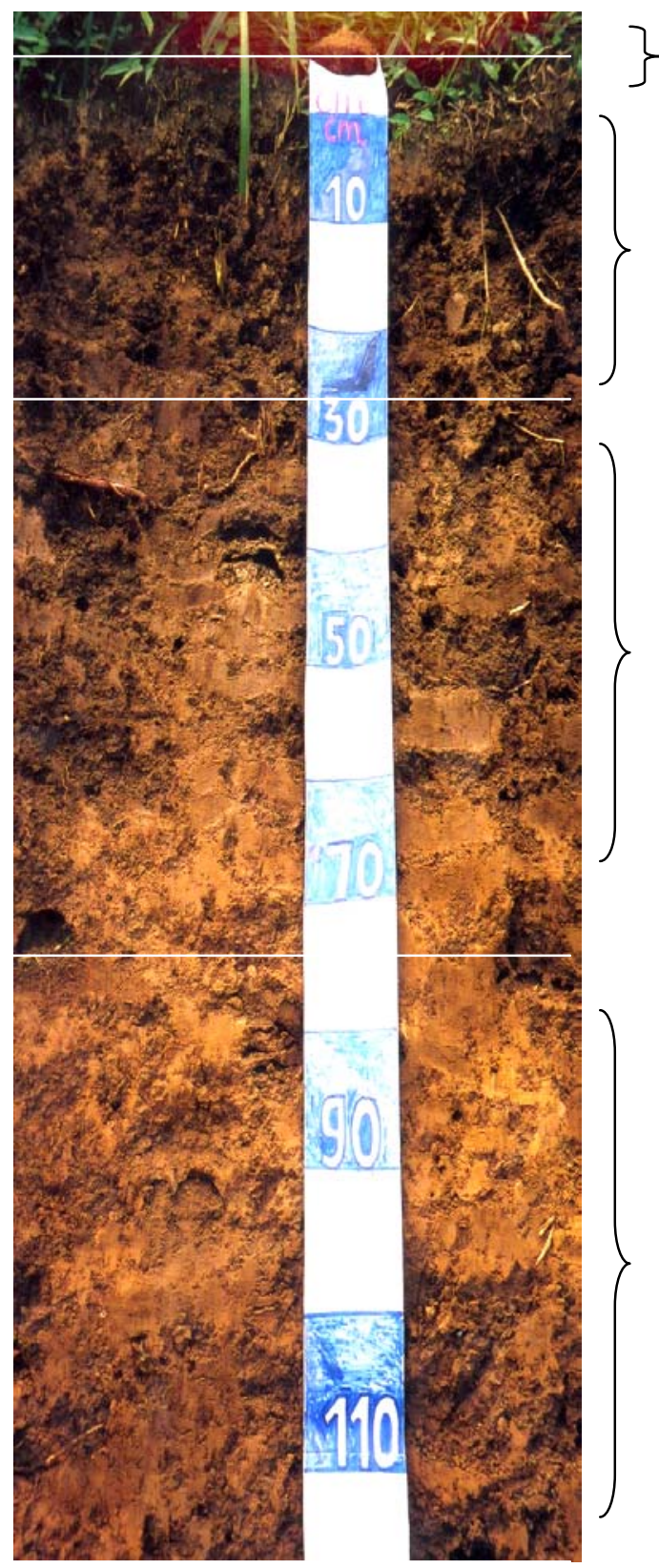

L:Laubstreu und organische Feinsubstanz

Ah: 0-28 cm: Farbe: feldfeucht - stark dunkelbraun (7,5 YR 3/3),

lufttrocken - matt gelb-braun (10YR 5/6), toniger Lehm, stark humos, karbonatfrei, stark durchwurzelt bis $22 \mathrm{~cm}$ Tiefe, schwach steinig, kiesig, Krümelgefüge, deutliche annähernd lineare Horizontbegrenzung, dicht gelagert: geschätzte Lagerungsdichte $1,6-1,8$.

Bws1 28-75 cm: Farbe: feldfeucht dunkelbraun (7/5 YR 4/3), lufttrocken - gelb braun (10YR 6/6), mittel schuffiger Ton (uT) wenig humos, mäßig durchwurzelt, Wurzeldurchmesser 1-2 mm,

Bodenfeuchte: frisch, karbonatfrei, geschätzte Lagerungsdichte 1,4-1,6, Krümel- bis Subpolyedergefüge, undeutlicher Übergang zum nachfolgenden Horizont

Bws2 75-130 cm: Farbe: feldfeucht gelbbraun (10 YR 5/6), lufttrocken hellgelb Braun (10 YR 6/8), schwach schluffiger Ton (uT), wenig durchwurzelt, geschätzte Lagerungsdichte $1,4-1,6$, Subpolyeder- und Polyedergefüge. 


\section{Probefläche Nr. 2 ( Nur Alang Alang Bewuchs)}

Geographische Lage: Ca. 6 km westlich von Dia Linh Gemeindezentrum

Höhe ü NN: 360m

Geologie: Kieseliger Schiefer, toniger Kalkstein, tuffig-sandiger Schluffstein.

Relief: konvexer Mittelhang; $25^{\circ}$ Hangneigung, Exposition S

Klima: s. Profil 1

Vegetation/ Nutzung: Grasbrache - dominierend Alang Alang (Imperata cylindrica P.B.), daneben Oxalis cornicllata L., vorher Anbau von Mais und Maniok

Bodentyp( FAO): Geric Ferralsol (FRg)

\section{Beschreibung Bodentyp Nr.2}

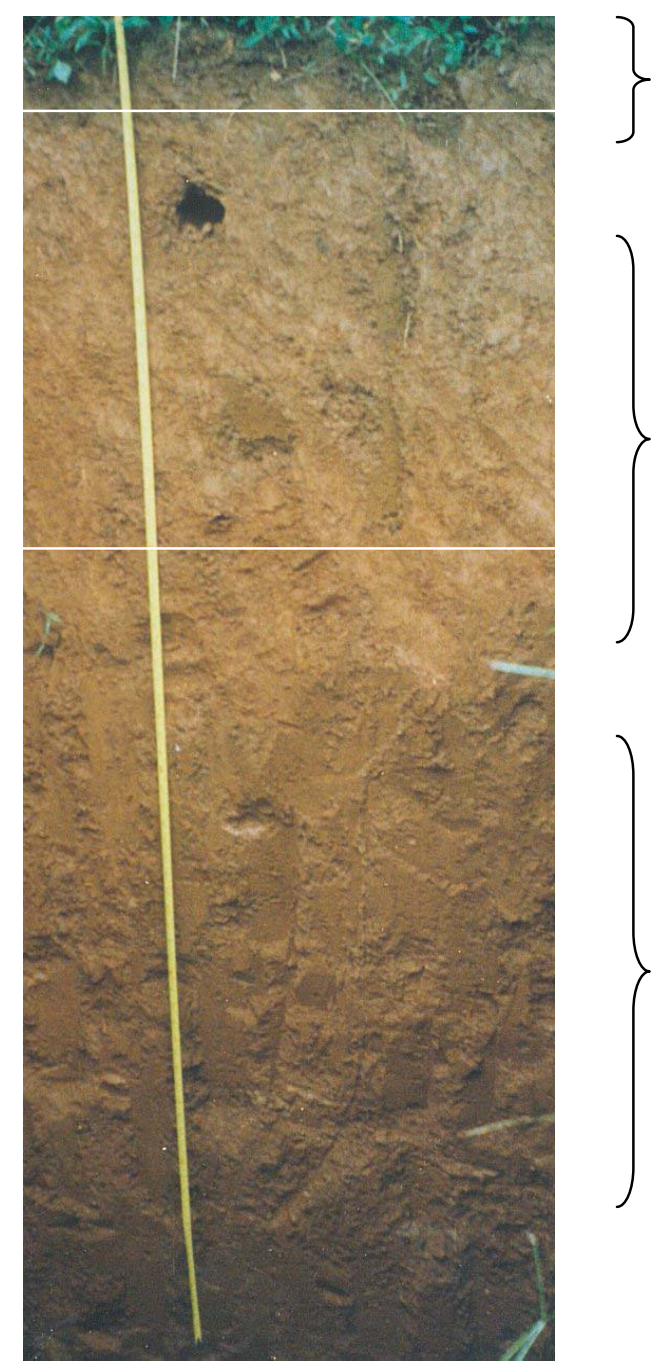

Ah (0-8cm): Farbe: feldfeucht - braun (10 YR 4/6), lufttrocken - hell gelb, orange (10YR 7/4) schluffiger Ton (uT), Bodenfeuchte: trocken, stark grusig (Quarz), 2\% Schotter und Kiese, stark durchwurzelt von Alang Alang, Polyedergefüge, karbonatfrei, deutliche Horizontbegrenzung.

Bws1 (8-50cm): Farbe: feldfeucht - mattbraun (7,5 YR 5/4), lufttrocken - matt orange (7,5 YR 7/4), stark schluffiger Ton (uT), Bodenfeuchte: feucht, geschätzte Lagerungsdichte 1,6 - 1,8. mittelsteinig - kiesig (20\%), stark durchwurzelt, Prismen- und grobes Polyedergefüge, undeutliche Horizontbegrenzung

Bws2 (50- $110 \mathrm{~cm}$ ): Farbe: feldfeucht - hell braun (7,5 YR 5/6), lufttrocken: hell gelb orange (7,5 YR 8/4), schwach schluffiger Ton (uT), Bodenfeuche: feucht, geschätzte Lagerungsdichte 1,6-1,8, stark kiesig - grusig (30-50\%), noch stark durchwurzelt, Polyedergefüge, Horizont in sich sehr homogen. 


\section{Probefläche Nr. 3 ( 1. Jahr nach Brandrodung)}

Geographische Lage: Ca. 6 km westlich von Dia Linh Gemeindezentrum

Höhe ü NN: $380 \mathrm{~m}$

Geologie: Kieseliger Schiefer, tuffig-sandiger Schluffstein, toniger Kalkstein .

Relief: konvexer Mittelhang

Klima: s. Profil 1

Vegetation/ Nutzung: Mais

Hydrologie: grundwasserfern

Bodentyp (FAO): Rhodic Ferralsol (FRr)

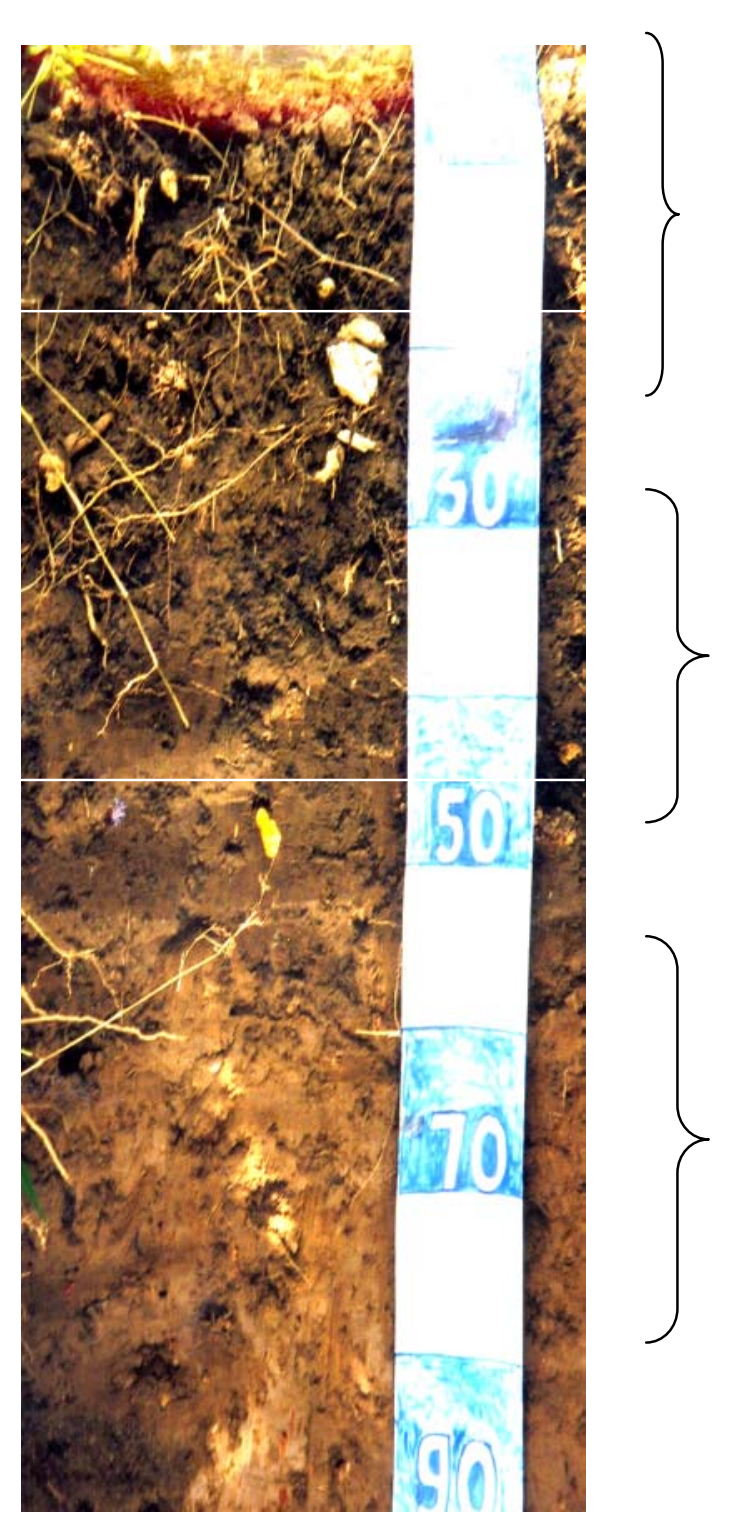

Ap (0-20 cm): Farbe: feldfeucht braunschwarz (10 YR 2/2), lufttrocken gelbbraun (10 YR 5/3), toniger Lehm (tL), schwach steinig, kiesig, grusig, Krümelgefüge, geschätzte Lagerungsdichte 1,4 - 1,6, Probe zerfällt nur in wenige Bruchstücke, die von Hand weiter zerteilt werden können, sehr locker, stark durchwurzelt, stark humos, undeutliche Horizontbegrenzung

Bws1 (20-45 cm): Farbe: feldfeucht matt gelb braun (10 YR 4/3), lufttrocken matt gelb orange (10 YR 6/4), toniger Lehm ( $\mathrm{tL})$, Subpolyedergefüge und Polyedergefüge, geschätzte Lagerungsdichte 1,4-1,6, Bodenfeuchte: feucht, stark durchwurzelt, humos, undeutliche Horizontbegrenzung.

Bws2 (45-95 cm) Farbe: feldfeucht Braun (10 YR 4/4 ),lufttrocken matt gelb orange (10YR 6/3), toniger Lehm (tL), Krümel-und Subpolyedergefüge,

geschätzte Lagerungsdichte 1,4 - 1,6 Bodenfeuchte: feucht, mitteldurchwurzelt, steinig (20\%) 


\section{Probefläche Nr. 4 ( 4. Jahr nach Brandrodung)}

Geographische Lage: Ca. 6 km westlich von Dia Linh Gemeindezentrum, an Probefläche

Nr. 3 angrenzend

Höhe über NN: 336m

Geologie: Kalkstein-Linsen, toniger Kalkstein, Tonschiefer.

Relief: konvexer Mittelhang; Hangneigung $23^{\circ}$

Klima: s. Profil 1

Vegetation/ Nutzung: Mais, sich ausbreitende Brachevegetation mit Alang alang

(Imperata cylindrica), Calamus rudentum, Calamus tennis, Eupatorium Odoratum L.,

Ageratum Cengzoides L.

Hydrologie: grundwasserfern

Bodentyp (FAO): Geric Ferralsol (FRg)

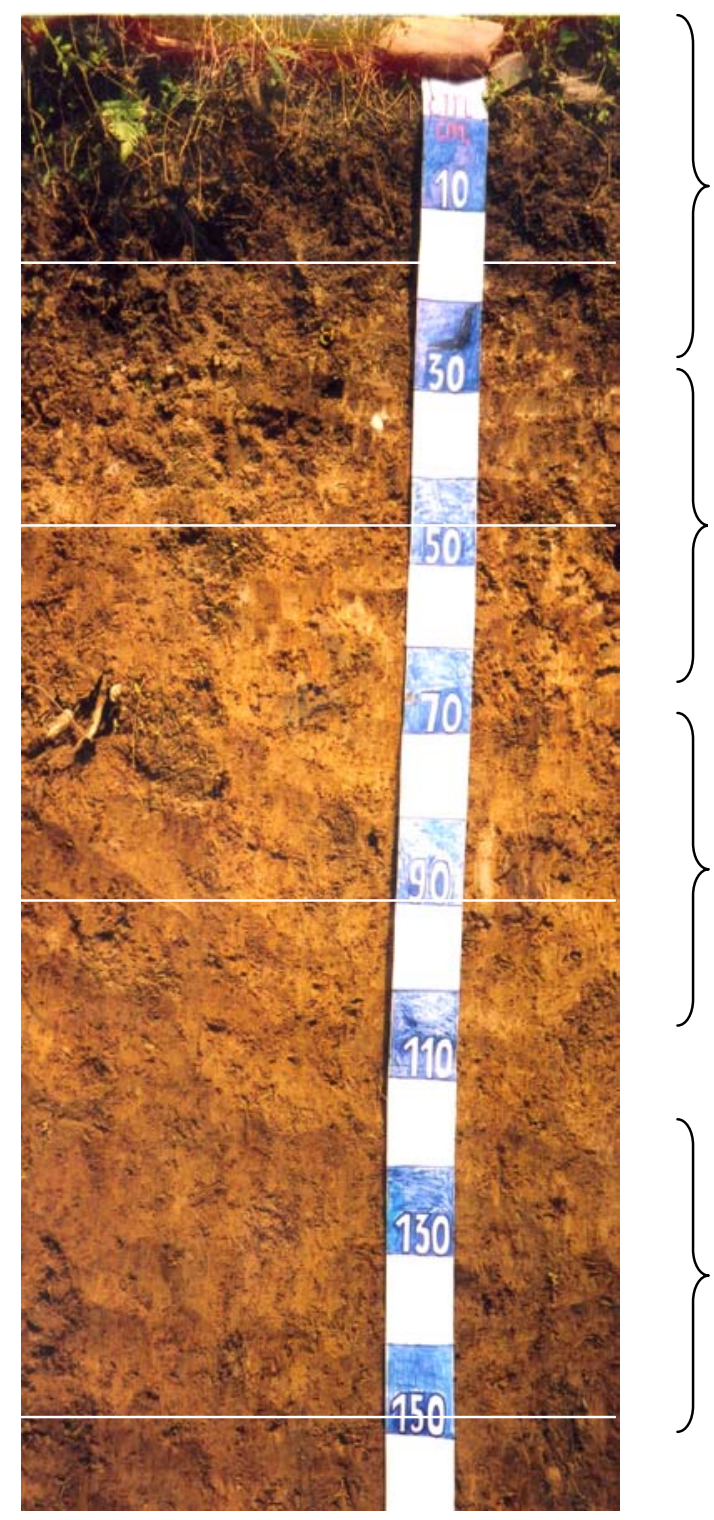

Ap (0-17 cm): Farbe: feldfeucht braunschwarz (10 YR 2/3), lufttrocken, matt gelb braun (10 YR 4/3), schluffig toniger Lehm (utL), Krümelgefüge, geschätzte Lagerungsdichte 1,4 - 1,6, feucht: nicht verklebt; nass: verklebt, locker, stark durchwurzelt mit Durchmesser 1-3 mm, schwach steinig (10\%), mit eckigen kleinenen Steinen von $2-4 \mathrm{~cm}$, humos, deutliche Horizontbegrenzung.

Bws1 (17-43cm): Farbe: feldfeucht hellgelb-braun (10 YR 6/8), lufttrocken matt gelb orange (10 YR 6/4), sandig- schluffiger Lehm (suL), Krümelgefüge und Subpolyedergefüge, wenn trocken: sehr hart, feucht : nicht verklebt, mittel durchwurzelt, mittelsteinig (20-30\%), undeutliche Horizontbegrenzung.

Bws2 (43-90 cm): Farbe: feldfeucht mattgelb-braun (10YR 4/3), lufttrocken matt gelb orange (10YR $6 / 3$ ), schluffig toniger Lehm (utL), Krümel- und Subpolyedergefüge, eckig, trocken : nass: zäh, geschmeidig, stark steinig mit Durchmessern von $2-30 \mathrm{~cm}$, in $50 \mathrm{~cm}$ Tiefe dünne Steinlage, undeutliche Horizontbegrenzung.

Bws3 (90-150 cm): Farbe: feldfeucht hellbraun (10 YR 4/6), lufttrocken hell gelb braun (10 YR 6/6), schwach toniger Lehm (tL), Krümel- und Subpolyedergefüge, wenn trocken : fest, hart; feucht : nicht verklebt, stark steinig mit Durchmessern von $5-20 \mathrm{~cm}$ 


\section{Probefläche Nr.5: 4. Brachejahr (Sekundärwald)}

Geographische Lage: Ca. 6 km westlich von Dia Linh Gemeindezentrum, an Probefläche

Nr. 3 angrenzend

Höhe über NN: $310 \mathrm{~m}$

Geologie: :Tonschiefer, toniger Kalkstein im Untergrund, Kalkstein an der Oberfläche

Hangneigung: $22-25^{\circ}$

Klima: s. Profil 1

Vegetation: Buschbrache bis 2 m Höhe mit Eupatorium Odoratum L., Chromolaene

Odotata Canarium album, Castanapsis sinensis , Calamus rudentum, Calamus tennis

Bodentyp( FAO): Rhodic Ferralsol (FRr)

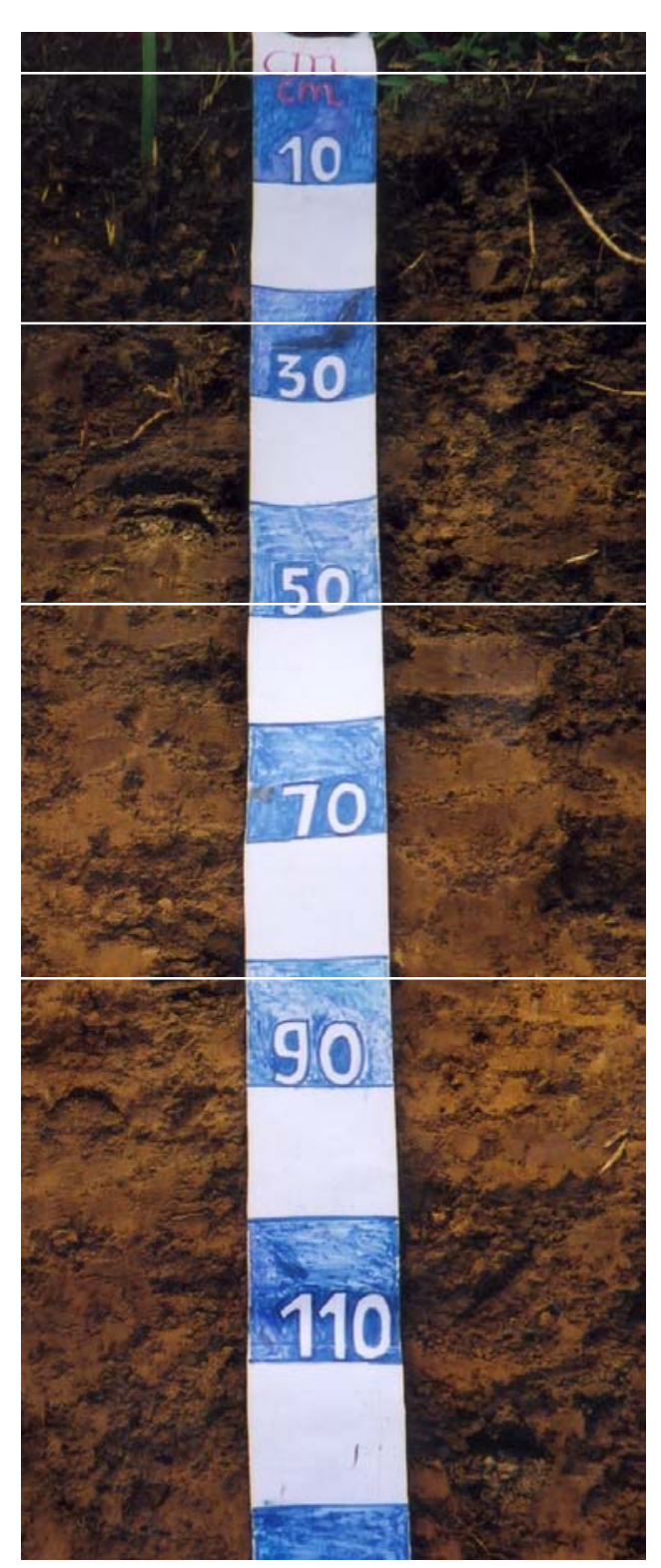

L:Laubstreu und organische Feinsubstanz

Ah (0-22cm): Farbe: feldfeucht braunes Schwarz (10 YR 2/2), lufttrocken matt gelbbraun (10 YR 4/3), sandiger Lehm (sL), stark humos, Bodenfeuchte:feucht,Krümel-und Subpolyledergefüge, stark durchwurzelt mit Durchmessern von 1-3 mm, schwach steinig-kiesig, deutliche Horizontbegrenzung.

Bws1 (22-50cm): Farbe: feldfeucht braunes Schwarz (10YR 3/2), lufttrocken matt gelbbraun (10 YR 5/3), toniger Lehm, Subpolyeder- und Polyedergefüge, geschätzte Lagerungsdichte 1,4-1,6, wenn trocken: hart und verklebt, wenn feucht : nicht verklebt, wenig durchwurzelt, undeutliche Horizontbegrenzung

Bws2 ( 50-82cm): Farbe: feldfeucht mattgelb-braun (10YR 4/3), lufttrocken hell gelbbraun (10YR 6/6), schwach schluffiger Ton, Subpolyeder- und Polyedergefüge, wenn trocken: hart, wenn feucht :verklebt, deutliche Horizontbegrenzung.

Bws3 (82-120cm): Farbe: feldfeucht gelbbraun (10 YR 5/6 M), lehmiger Ton, Prismenund Polyedergefüge, schwach steinig mit Durchmessern von 3-10 cm. 


\section{Probefläche Nr. 6 ( Sekundärwald)}

Geographische Lage: Ca. 6 km westlich von Dia Linh Gemeindezentrum

Höhe ü NN: 260m

Geologie: Kieseliger Schiefer, toniger Kalkstein, tuffig-sandiger Schluffstein.

Relief: konvexer Mittelhang; $25^{\circ}$ Hangneigung, Exposition S

Klima: s. Profil 1

Vegetation/ Nutzung: Grasbrache - dominierend Alang Alang (Imperata cylindrica P.B.), daneben Oxalis cornicllata L., vorher Anbau von Mais und Manio

Bodentyp (FAO): Humic Ferralsol (FRu)

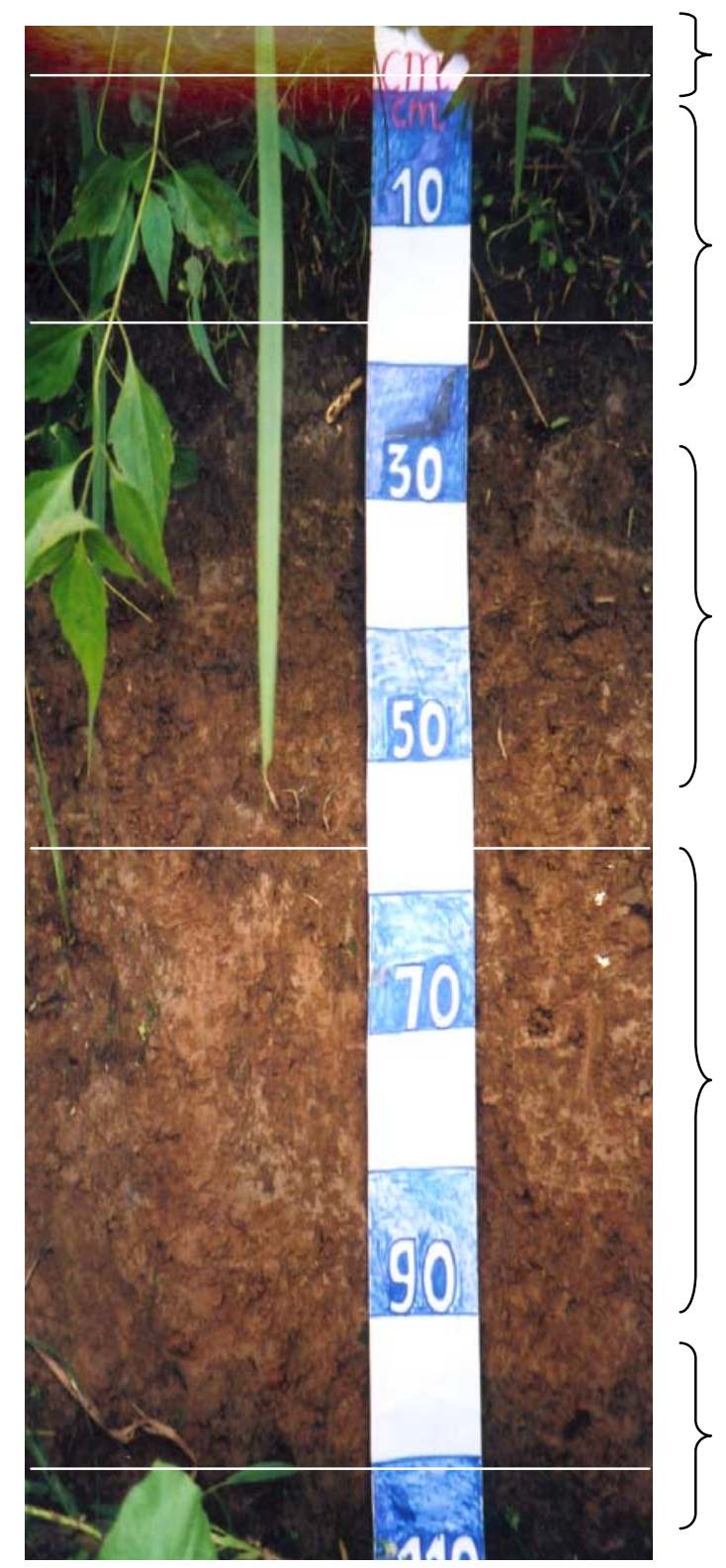

L:Laubstreu und organische Feinsubstanz

Ah (0-18cm): Farbe:feldfeucht braunes Schwarz (10YR 5/3), lufttrocken gelbbraun (10 YR 4/3), sandiger schluffiger Lehm( $\mathrm{uL})$, stark humos,

stark durchwurzelt mit durchmessern von 2-4 $\mathrm{mm}$.

Bws1(18-55cm): Farbe: feldfeucht hellgelb-braun (10YR 6/7), lufttrocken matt gelb-braun (10 YR6/6), schwach schuffiger Ton, Subpolyedergefüge, geschätzte Langerungsdichte 1,4-1,6, undeulichle Horizontbegrenzung.

Bws2 (55-100cm): Farbe.feldfeucht Braun (10 YR 4/4), lufttrocken matt gelb orange(10 YR 6/3), toniger Lehm,Krümelund Subpolyedergefüge geschätzte Langerungsdichte 1,4-1,6 schwach steinig, kiesig, deutliche Horizontbegrenzung

Bws3 (100-105cm): Farbe: feldfeucht hellbraun (10 YR 4/6), lufftrocken gelb orange (10 YR 6/3). Schwach steinig mit Durchmessern von $4-7 \mathrm{~cm}$. 
Abb. 18: Verteilung der Leitbodenprofile bei neuem Landnutzungssystem (Agroforstsystem mit Mulch)

Legende s. Anhang A. verändert nach BARSCH; BILLWITZ

\& BORK, 2000
Agroforstsystem mit Mulch

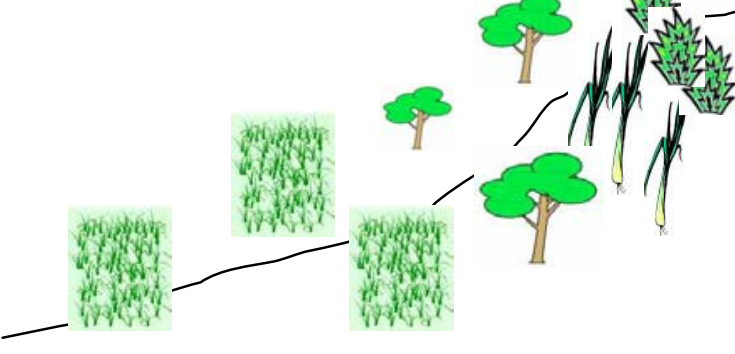

Gartenbaut und Nassreis

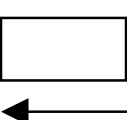

\section{Geric Ferralsol (FRg)}

$120 \perp$

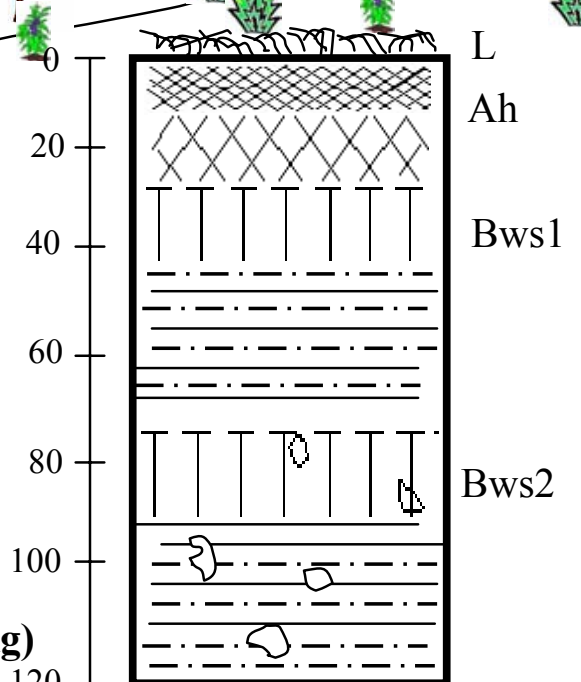

Alang alang
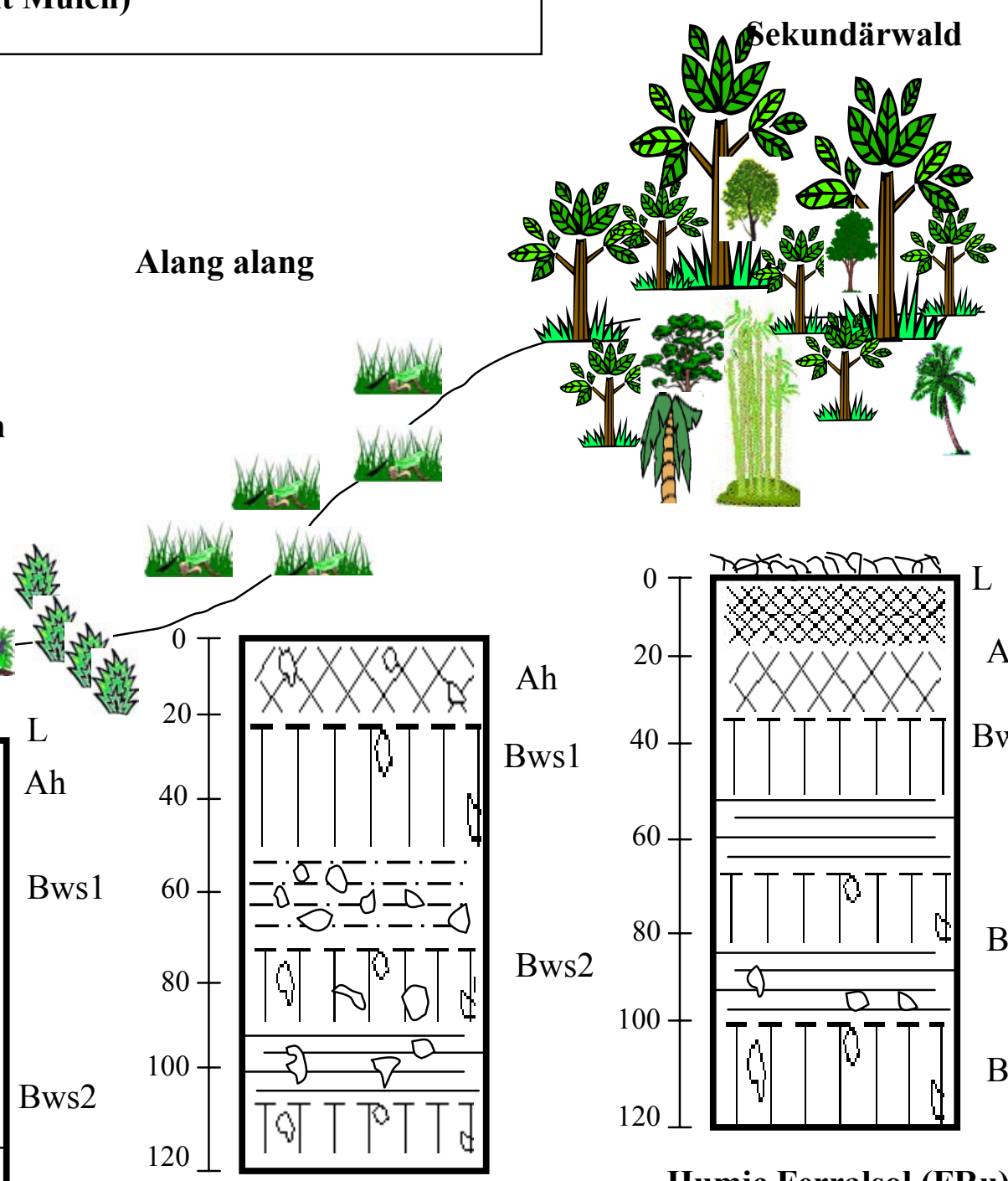

$\mathrm{Ah}$

Geric Ferralsol (FRg)

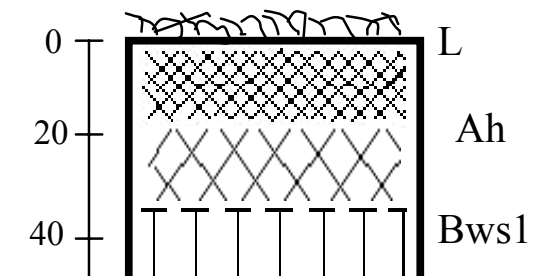

Humic Ferralsol (FRu)

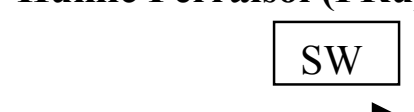


Abb. 17: Verteilung der Leitbodenprofile bei traditionellem Landnutzungssystem

(Wanderfeldbaum mit Brandrodung)

Legende s. Anhang A. verändert nach BARSCH; BILLWITZ

\& BORK, 2000

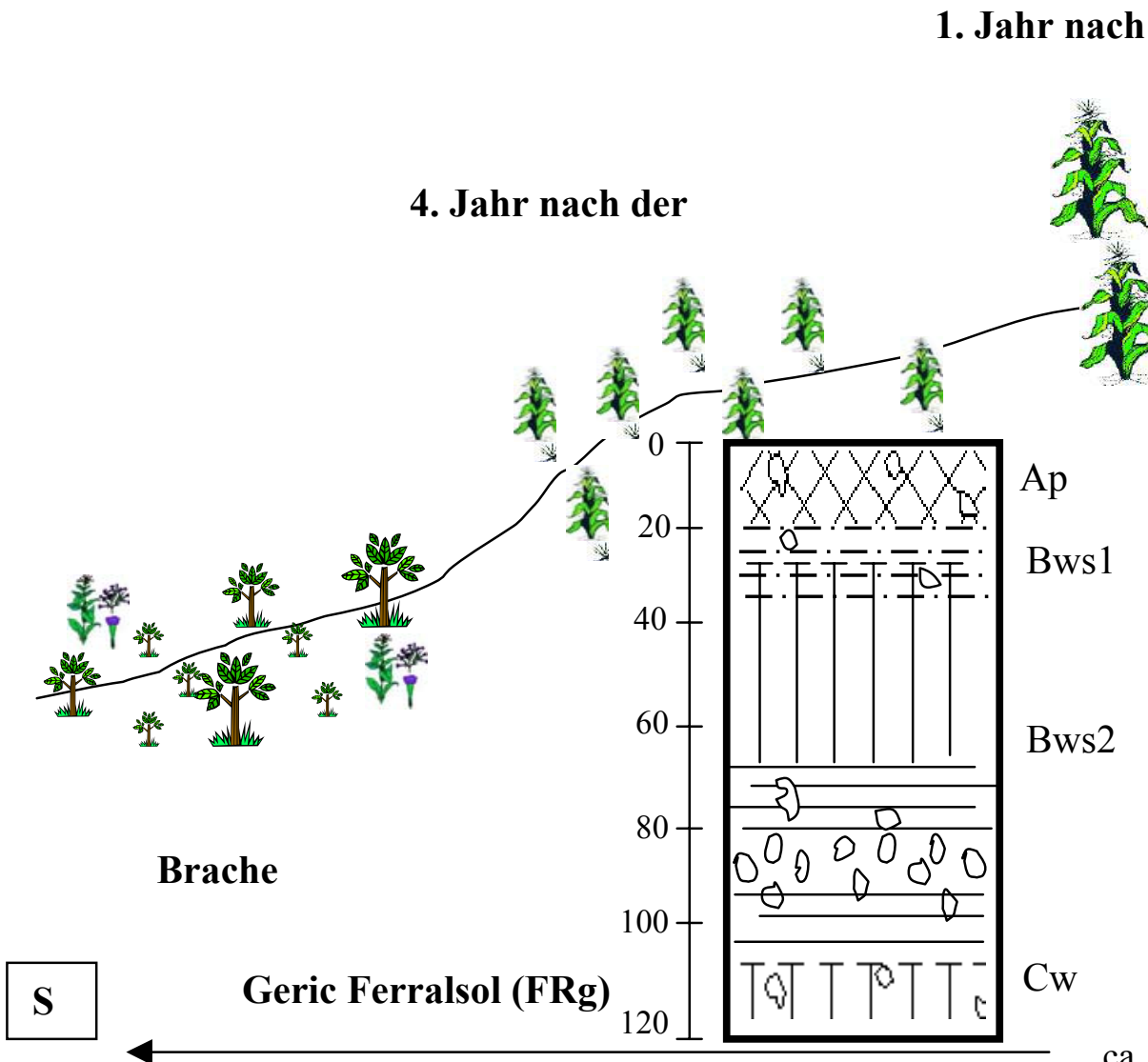

\section{er Brandrodung}
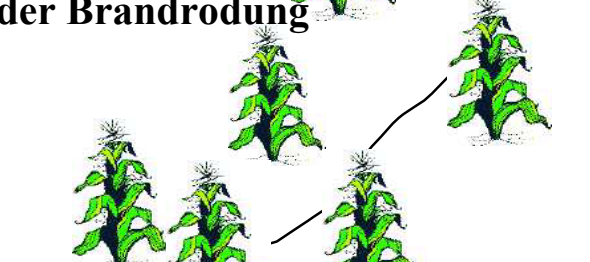$$
\text { 西 }
$$
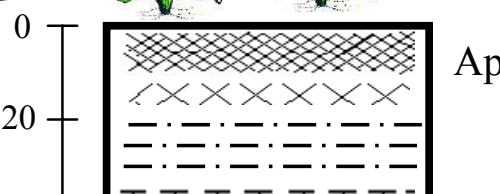

Ap

$40-$

三:二:三:二:三:二$$
40-\top \mathrm{T} T \mathrm{~T} \mathrm{~T}^{-}
$$$$
60-
$$

80

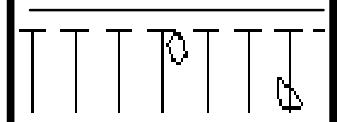

100

120

Rhodic Ferralsol (FRr)
4. Brachejahr Sekundärwald 420 牙
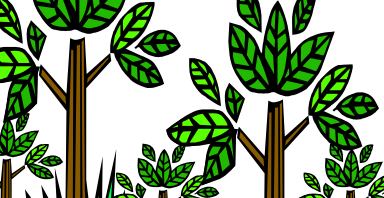

\section{(1)}

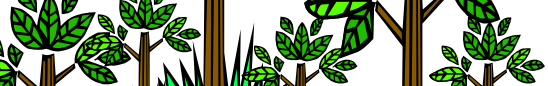

詸

WW

1
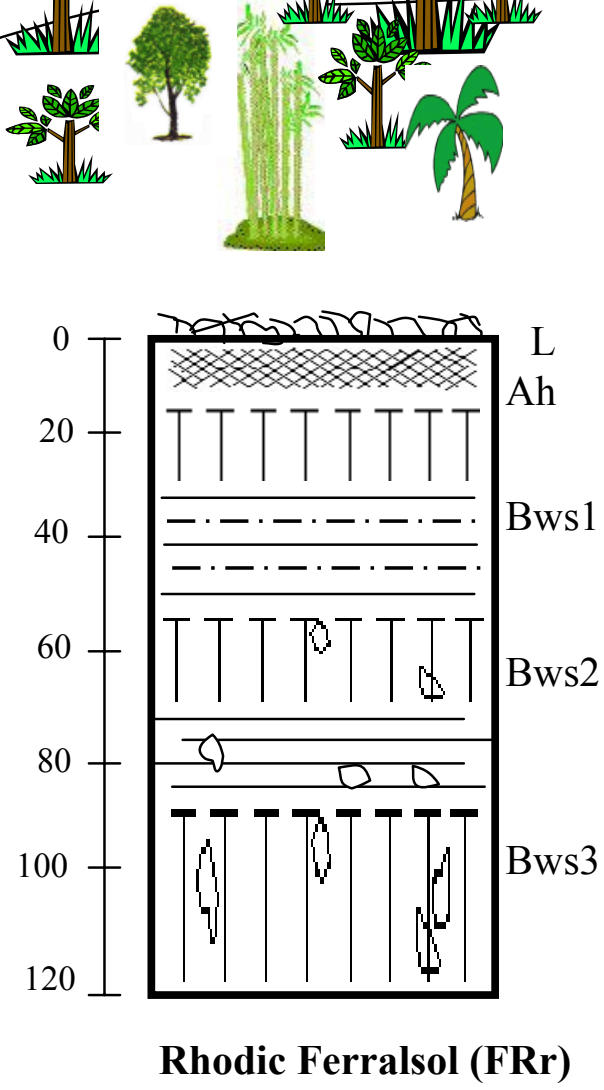

ca. $600 \mathrm{~m}$

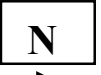




\subsection{Bodeneigenschaften und Bodenqualität im Untersuchungsgebiet}

\section{Interdependenz wichtiger Parameter:}

Viele Bodenparameter stehen in einem kausalen Zusammenhang zueinander, d.h. sie beeinflussen sich in positiver oder negativer Weise. In den vorangegangen Schilderungen des Bodenzustandes wurden solche Parameterbeziehungen herausgestellt. Für einen zusammenfassenden Überblick sollen an dieser Stelle noch einmal einige aussagekräftige Korrelationen in Diagrammen verdeutlicht werden.

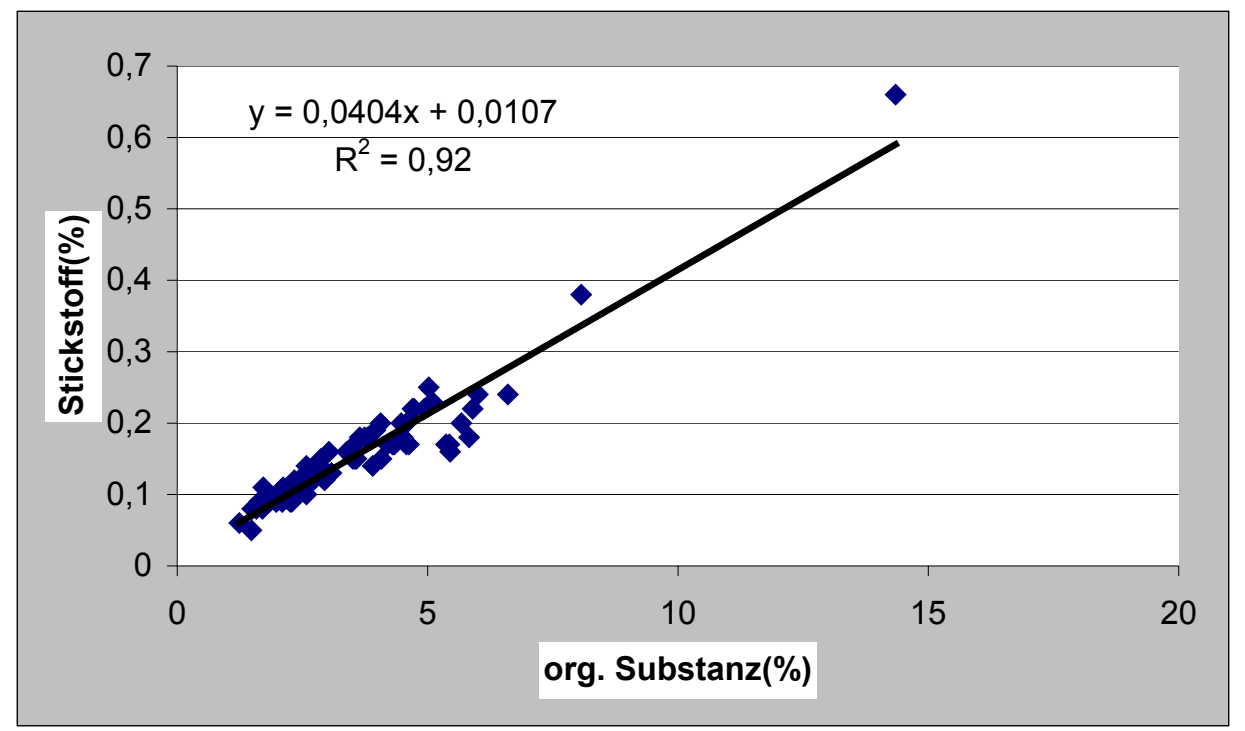

\section{Abb. 19: Korrelation zwischen organischer Substanz und Gesamtstickstoff}

Die organische Substanz hat auf die Nährstoffversorgung entscheidenden Einfluss, da der Hauptnährstoff der Pflanzen, Stickstoff, zu 95\% an Humus gebunden ist. Die enge Korrelation zwischen organischer Substanz und Stickstoff wird in der Abb. 19 deutlich. Der Gesamt $-\mathrm{N}$-Gehalt des Bodens steht in enger Beziehung zum Gehalt an organischer Substanz bzw. zum C-Gehalt (PAGEL 1982).

Die Werte der effektiven Kationnenaustauschkapazität (KAKeff) steigen proportional mit der Zunahme organischer Substanz im Boden. Das Bestimmtheitsmaß $\left(r^{2}=0,60\right)$ verdeutlicht noch einmal die Fähigkeit der Huminstoffe zur Kationenadsorption und damit ihre Funktion als bedeutende Nährstoffträger. MÜLLER-SÄMANN (1986) ermittelte zwischen dem Gehalt der Böden an organischer Substanz und der Austauskapazität eine hoch signifikante, sehr enge Korrelation $(\mathrm{r}=0,988)$. 


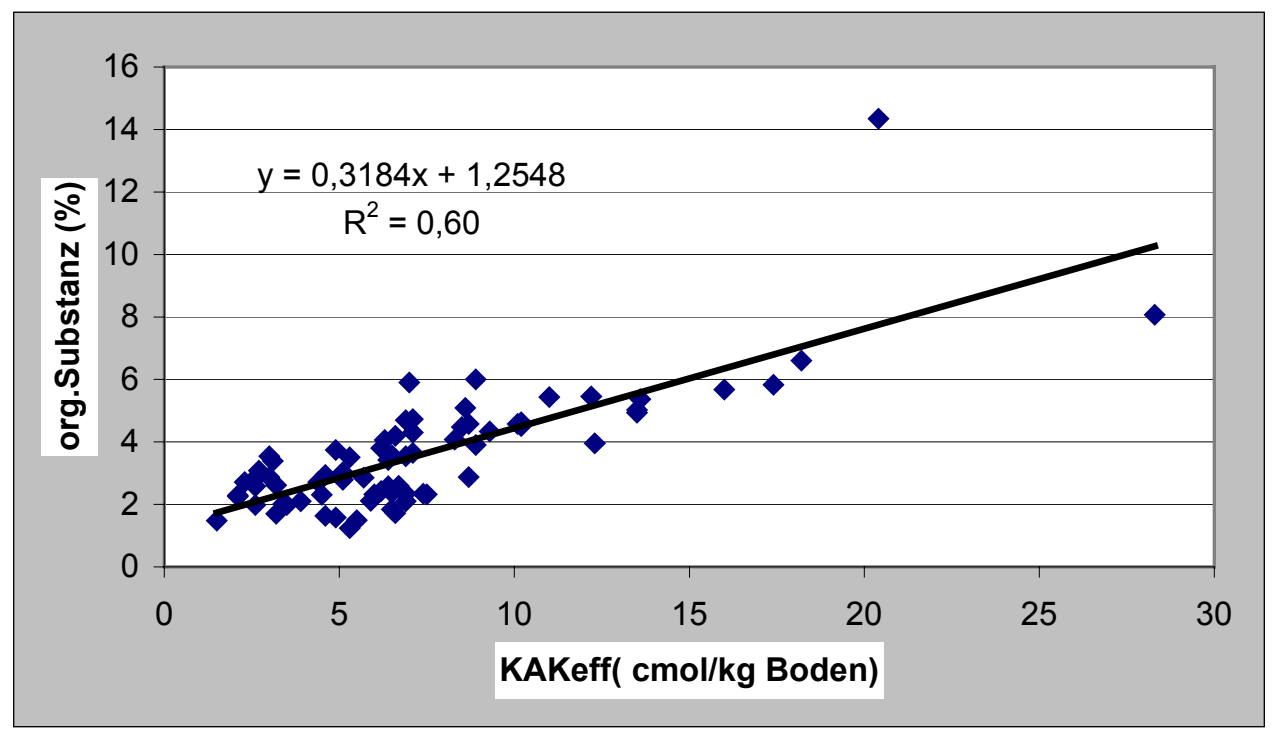

Abb. 20 : Korrelation zwischen organischer Substanz und KAKeff

Die Korrelation zwischen pH-Wert und KAKeff ergab eine stark positive Beziehung zwischen beiden Parametern $(r=0,78)$. Nach PAGEL (1974) können in begrenzten pHBereichen deshalb häufig enge Beziehungen zwischen $\mathrm{pH}-$ Werten und allgemeinen chemischen Bodeneigenschaften bzw. Gehalten an verfügbaren und anderen Nährstoffformen nachgewiesen werden.

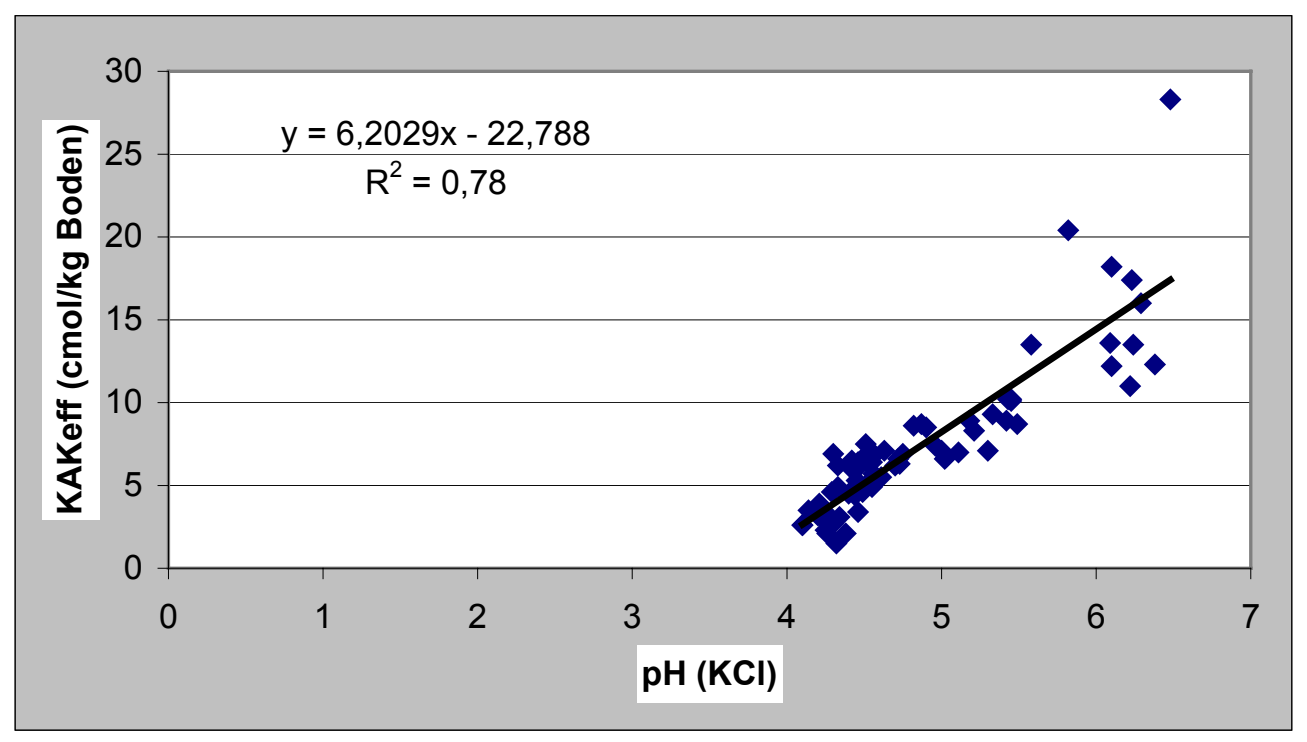

Abb. 21: Korrelation zwischen pH (KCl) und KAKeff

Der pH-Wert wird auch zur Beurteilung weiterer chemischer Bodeneigenschaften herangezogen. PAGEL (1981) ermittelte in Nordvietnam eine positive signifikant Korrelation zwischen pH-Wert und KAKeff an 380 Bodenproben. Die Kationenaustauschkapazität wird bedingt durch die Art und Mengenanteile der 
Tonminerale und Huminstoffe, wobei die KAK mit steigendem pH-Wert normalerweise zunimmt, da die Huminstoffe eine variable Ladung besitzen (AG BODEN 1994).

Enge Korrelationen zwischen dem Sorptionskoeffizienten und dem Gehalt an organischer Substanz des Bodens konnten nachgewiesen werden. (SCHINNER \& SONNLEITNER 1997). Bodeneigenschaften wie $\mathrm{pH}-$ Wert sowie die Tonqualität und- quantität besitzen bei Böden mit hohem organischen Substanzgehalt einen geringeren Einfluss auf die Sorptionskapazität.

Nach BECKER ET AL (1999) sinkt das Verhältnis von KAKeff zu KAKpot im pH Bereich zwischen 7 und 5 von 1 auf etwa 0,85 (abhängig vom Anteil pH-variabler Ladung und von der Ionenstärke der Bodenlösung). Unterhalb von $\mathrm{pH} 5$ sinkt es jedoch rapide ab (bei pH 3 z.B auf 0,29).

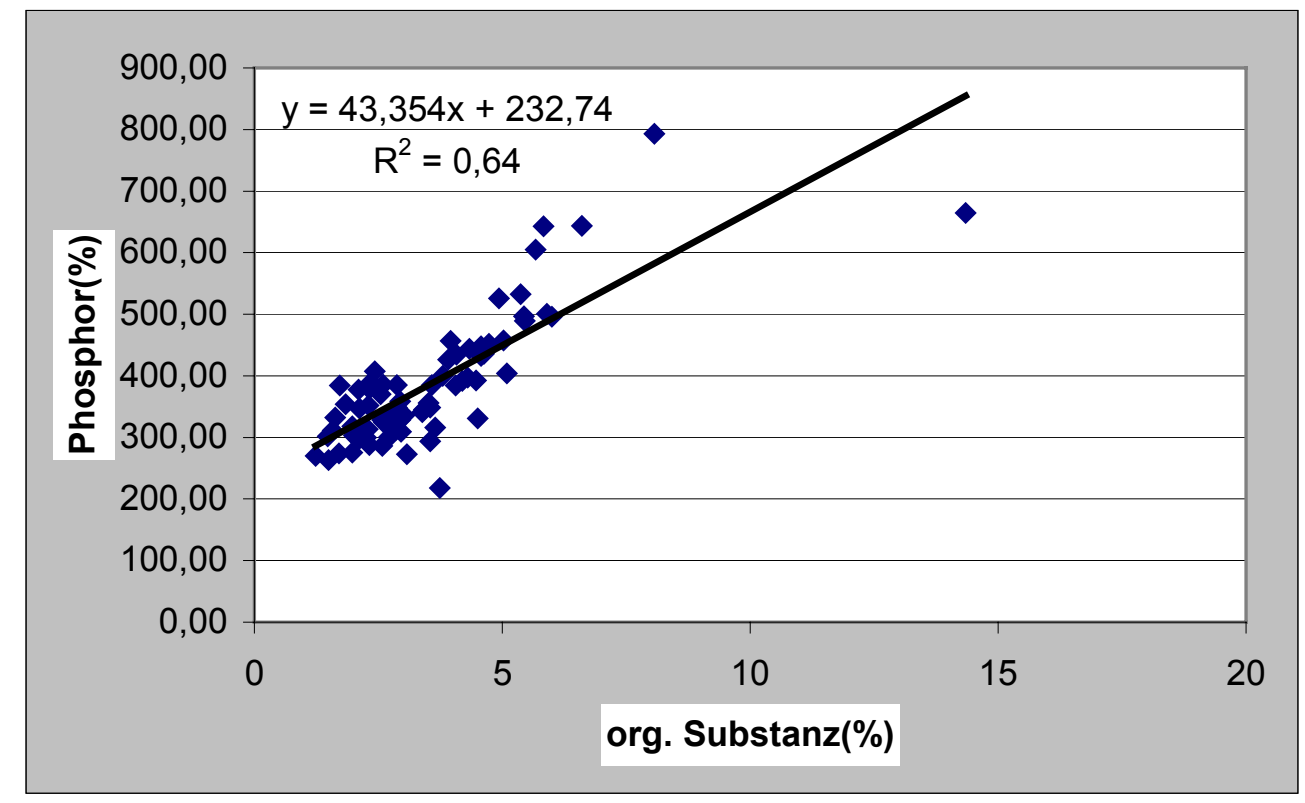

\section{Abb. 22 : Korrelation zwischen Phosphor (Pt) und org. Substanz}

Zwischen organischer Substanz und Phosphor besteht ein enger Zusammenhang, der aus dem hohen Korerationskoeffizienten $(\mathrm{r}=0,64)$ ersichtlich ist. Da mehr als 64\% des gesamten P-Vorrates der untersuchten Böden in organischer Form vorliegen, stellt der Humus einen sehr bedeutenden P-Speicher dar. Hohe biologische Aktivität in den Böden fördert die Phosphatmobilisierung entscheidend.

In Ferallitischen Boden lassen sich bei niedrigen pH- Werten enge Beziehungen zwischen Phosphorverfügbarkeit, Eisen und Aluminium ableiten. Die Beziehungen zwischen pH, Sättigungsgrad, und KAKeff hängen von vielen Faktoren ab. Da das Pflanzenwachstum 
auf sauren Böden primär durch das Aluminium begrenzt wird, sind die Austauschazidität bzw. der Sättigungsgrad des Bodens die wichtigeren Größen zur Beurteilung der Bodenfruchtbarkeit. Infolge der festeren Bindung von $\mathrm{H}^{+}$und $\mathrm{Al}^{+++}$, aber lockereren Bindung von $\mathrm{Ca}^{++}, \mathrm{Mg}^{++}$und anderen basenbildenden Kationen an der $\mathrm{pH}$-abhängigen Ladung, ist der optimale Basensättigungsgrad in Böden mit hohem Anteil pH-abhängiger Ladung niedriger, der entsprechende Al-Sätigungsgrad also höher als in Böden mit vorwiegend permanenter Ladung. EVAN \& KAMPRATH (1970) ermittelten z.B., daß der Al - sättigungsgrad für gutes Wachstum von Mais $<45 \%$, Sojabohnen $<20 \%$ betragen soll.

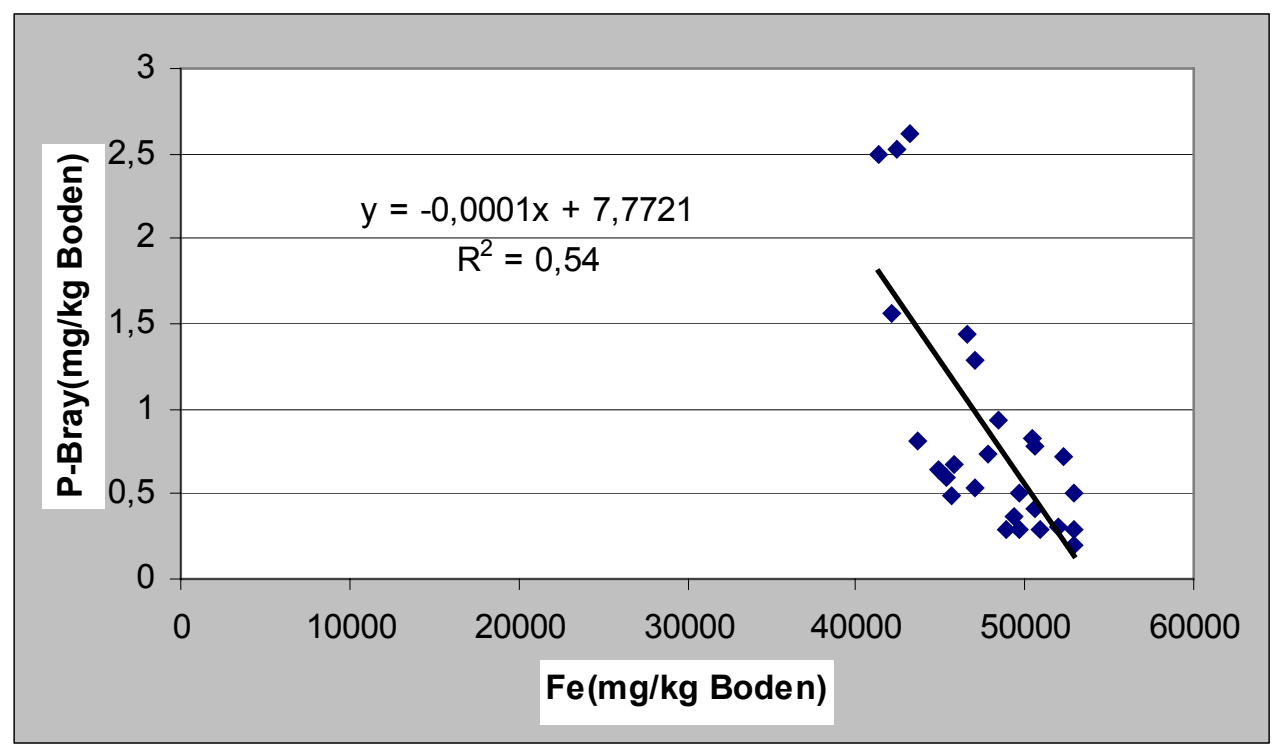

Abb.23: Korrelation zwischen Phosphorverfügbarkeit und Fe

Die Korrelation zwischen Phosphorverfügbarkeit, Al und Fe ergab enge Beziehungen zwischen diesen Parametern. Das Bestimmtheitsmaß von 0,54 zeigt eine starke Streuung an, wobei eine leichte Tendenz $\mathrm{zu}$ erkennen ist, dass die Erhöhung des Fe-und Aluminiumgehaltes eine Abnahme des verfügbaren Phosphors bewirkt. Mit steigender Fe - und Aluminiumtoxität nimmt der Anteil des pflanzen verfügbaren Phosphats ab. (THAI PHIEN ET AL1999) 


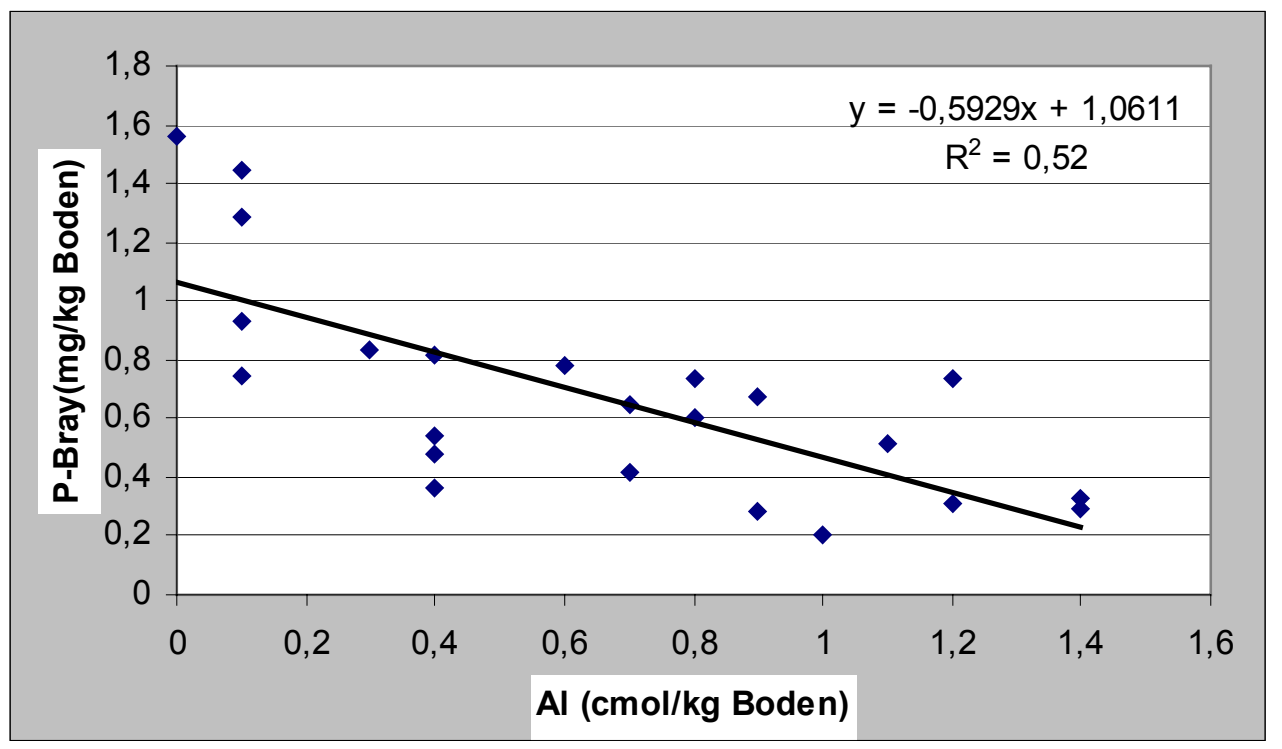

Abb.24: Korrelation zwischen Phosphorsverfügbarkeit und Al

Folgende Charakteristika der untersuchten Böden ergeben sich aus den vorangegangenen Ausführungen:

1- Die Kationenaustauschkapazität der Böden ist gering, meist unter $8 \mathrm{cmol} / \mathrm{kg}$ )

2- Die Böden sind sauer, $\mathrm{pH}$-Werte meist unter $5(5,5)$.

3- Die Profile sind sehr tief, einheitlich verwittert und nach der FAO- Systematik haben die Böden einen B-Horizont, in dem Fe-Oxide angereichert sind.

4- Die Struktur und die Färbung der Böden zeigen im Profil keine deutliche Differenzierung.

5- Diese günstigen Eigenschaften werden kontrastiert durch die zum Teil geringe nutzbare Wasserkapazität und durch Nährstoffarmut. Neben $\mathrm{N}$ tritt insbesondere $\mathrm{P}$ Mangel und zum Teil auch Mangel an Mikronährstoffen auf. Außerdem treten Eisen-, Aluminium- und auch Mangantoxizität auf.

Annuelle Kulturen sind nur mit hohem Risiko anzubauen, da sie einen schnellen Verlust organischer Substanz (2 bis 5\% pro Jahr) nach sich ziehen, eine hohe Erosionsgefährdung aufweisen, und zum Verlust der Bodenfruchtbarkeit -schon nach 2 bis 3 Jahren- führen, die unter natürlicher Vegetation relativ gut sein kann. Diese Böden werden häufig für den Anbau genutzt. Sie erbringen nach 2 jähriger Bewirtschaftung aber nur geringe Erträge, wenn beim Ackerbau keine entsprechenden Erosions- und Humusschutzmaßnahme betrieben werden.

Längerfristig sind nur Buschbrachesysteme mit langen Brachezeiten oder waldähnliche Nutzungsformen möglich, die das enge Kreislaufsystem der Nährstoffe geschlossen 
halten und den Boden dauernd bedecken. Doch auch dann müssen die Nährstoffexporte gering gehalten werden. Lediglich der Export von Kohlehydraten scheint langfristig den ökologischen Verhältnissen angemessen (BUI, Q. T., 1990).

\subsection{Bodenfruchtbarkeitsparameter in Abhängigkeit von der Nutzungsdauer}

\subsection{1. pH und Basensättigung}

Die Bodenreaktion ist ein Indikator zahlreicher fruchtbarkeitsbestimmender oder beeinflussender chemischer, physikalischer und biologischer Bodeneigenschaften. Sie wird in der Regel durch den pH-Wert der wässrigen oder salzhaltigen Bodensuspension erfasst.

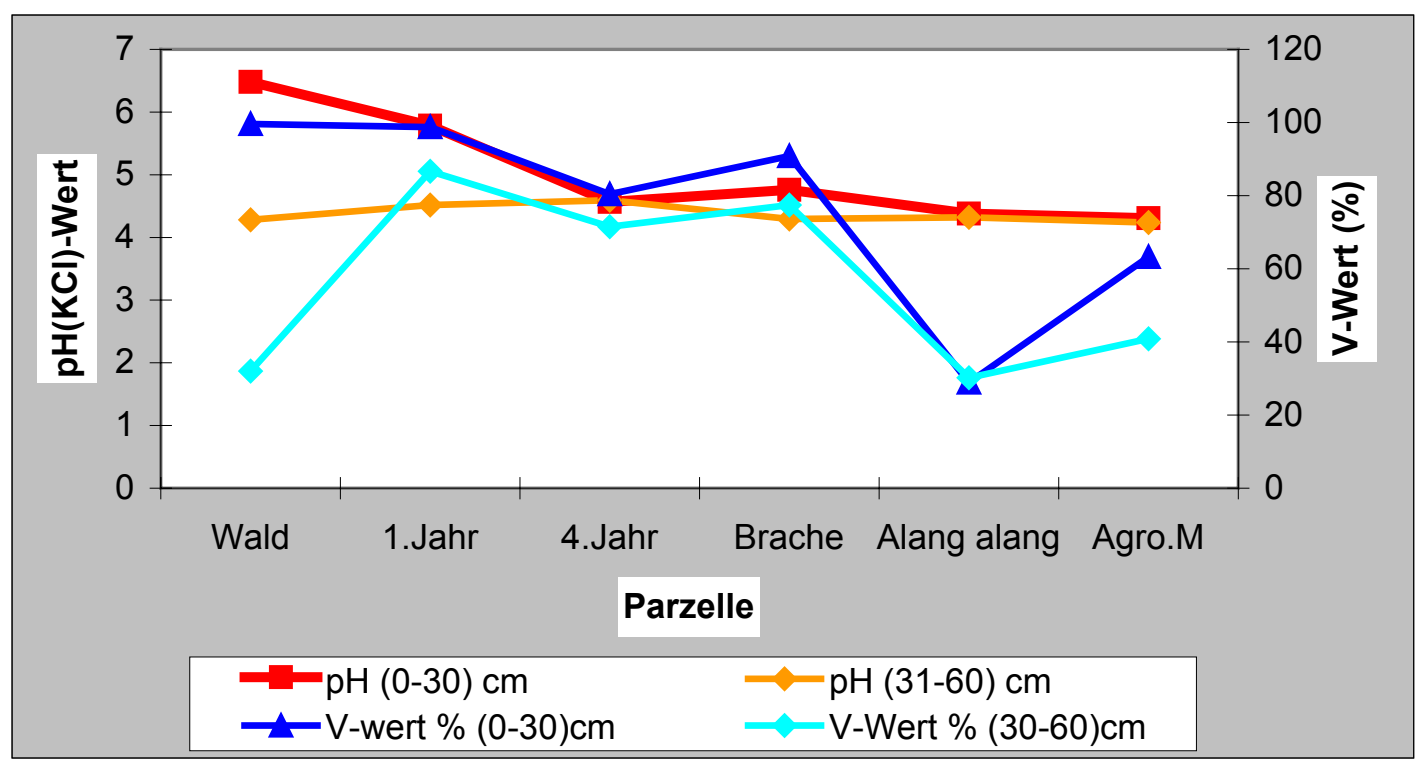

\section{Abb.25: pH-Wert und Basensättigung in Abhängigkeit von Anbaudauer und Nutzungstyp}

Darüber hinaus kommt es unter dem Einfluss der genannten Faktoren und verschiedener Landnutzungen $\mathrm{zu}$ unterschiedlichen $\mathrm{pH}$ - Werten und $\mathrm{zu}$ zeitlichen Veränderungen der Werte im Boden. Abb. 25 zeigt die Entwicklung der pH-Werte in Abhängigkeit von der Nutzungsdauer und Bodentiefe. Es ist eine deutliche Abnahme der $\mathrm{pH}-$ Werte mit Zunahme der Dauer des traditionellen Wanderfeldbaus zu verzeichnen. Der Oberboden $(0-30 \mathrm{~cm})$ der Waldparzelle erreicht einen $\mathrm{pH}-$ Wert von 6,5. Mit Beginn der landwirtschaftlichen Nutzung sinkt der pH-Wert deutlich von 6,5 auf 4,6 im 4. Jahr nach der Brandrodung. Im Unterboden $(30-60 \mathrm{~cm})$ schwankt der pH-Wert kaum. Sowie pH- 
Wert sinkt die Basensättigung deutlich von 99,6\% auf 80,4\% im 4.Jahr und sinkt dann weiter auf nur noch $28,9 \%$ in der Alang alang Parzelle.

Auf Böden mit niedrigem pH-Wert, ist das Pflanzenwachstum häufig gestört, die Ertragbildung ist gering, und in extremen Fällen sterben die Pflanzen ab. PAGEL (1981) nennt als wesentliche ungünstige Fruchtbarkeitsmerkmale saurer Böden:

- Hohe Mengen an austauschbarem und löslichem Al (z.T. auch an Mangan) bei pHWerten von 4,5 bis 5,0 die toxisch auf die Pflanzen wirken, geringe oder negative Wirkung zugeführter NPK- Düngemittel (Düngungsinaktivität).

- Mangel an verfügbarem $\mathrm{Mg}$ und $\mathrm{Ca}$, geringe Kationen-, aber hohe Anionensorption und - Fixierung, Tonmineralabbau, oft hoher Auswachungsgrad an Fe, Mn, Zn, Cu und B.

Bemerkenswert (Abb. 25) ist die sehr deutliche Abnahme von pH-Werte und Basensättigung nach der mehrjährigen landwirtschaftlichen Nutzung. Der $\mathrm{pH}-$ Wert sinkt von 6,5 auf 4,3, der V-Wert von 99,6\% auf 28,9\%. ein Anzeichen für die biologische Bodendegradation.

Bei niedrigen $\mathrm{pH}-$ Werten und schwacher Nährstoffversorgung gelingt keine ausreichende Produktion an Biomasse, so dass auch die Folgekulturen die Nährstoffe in der Biomasse nicht zu der notwendigen Ertragsteigerung verwenden können. Bei niedrigen pH-Wert und austauschbaren Al bereitet die steigende Phosphorfixierung Probleme. Dadurch wird das normale Pflanzenwachstum häufig gestört. Die Ertragsbildung ist sehr gering. Deswegen werden auf diesen Flächen keine landwirtschaftlichen Kulturen mehr angebaut und es kommt zur Ausbreitung des Alang Alang Bewuches. Nach den lokalen Kenntnissen der Bauern ist Alang Alang ein deutlicher Indikator für Bodendegradation. Nach Thai Phien \& Nguyen Tu Siem (1999) sind im Bergland von Vietnam auf $86 \%$ der Fläche sehr stark saure Böden ( $\mathrm{pH}-\mathrm{KCL} 4,5-4$, sogar 3,5) verbreitet und 70\% der Fläche weisen Hangneigungen von $>25^{\circ}$ auf. Als Folge der Bodenerosion sind 500000 ha Land geschädigt.

Der $\mathrm{pH}$ - Wert $(\mathrm{KCl})$ vor dem Anbau und nach dem Ernte bei nur einer zwischenliegenden Regenzeit gemessen, sinkt sehr deutlich von 5,8 auf 5,2 in der Parzelle des 1.Jahres nach der Brandrodung und 4,6- 4,0 im 4. Jahr nach der Brandrodung. Dagegen steigt $\mathrm{pH}-$ Wert $(\mathrm{KCl})$ von $4,3-$ auf 4,8 in der Parzelle des Agroforstsystems mit Mulch. Die Zunahme des $\mathrm{pH}$-Wertes ist bedingt durch die Nährstoffzufuhr mit dem Mulchmaterial.

Bei der Brache schwankt der pH-Wert kaum. Die Ursache während der Regenzeit liegt in dem Verlust an Kationen im Oberboden. Aus den Ergebnissen (Abb. 26) kann man 
abermals festgestellen, dass durch den traditionellen Brandrodungswanderfeldbau die Oberböden sehr schnell degradiert werden. Diese Ergebnisse sind ein deutliches Anzeichen dafür, dass richtiges Management eine Bodendegradation limitieren kann.

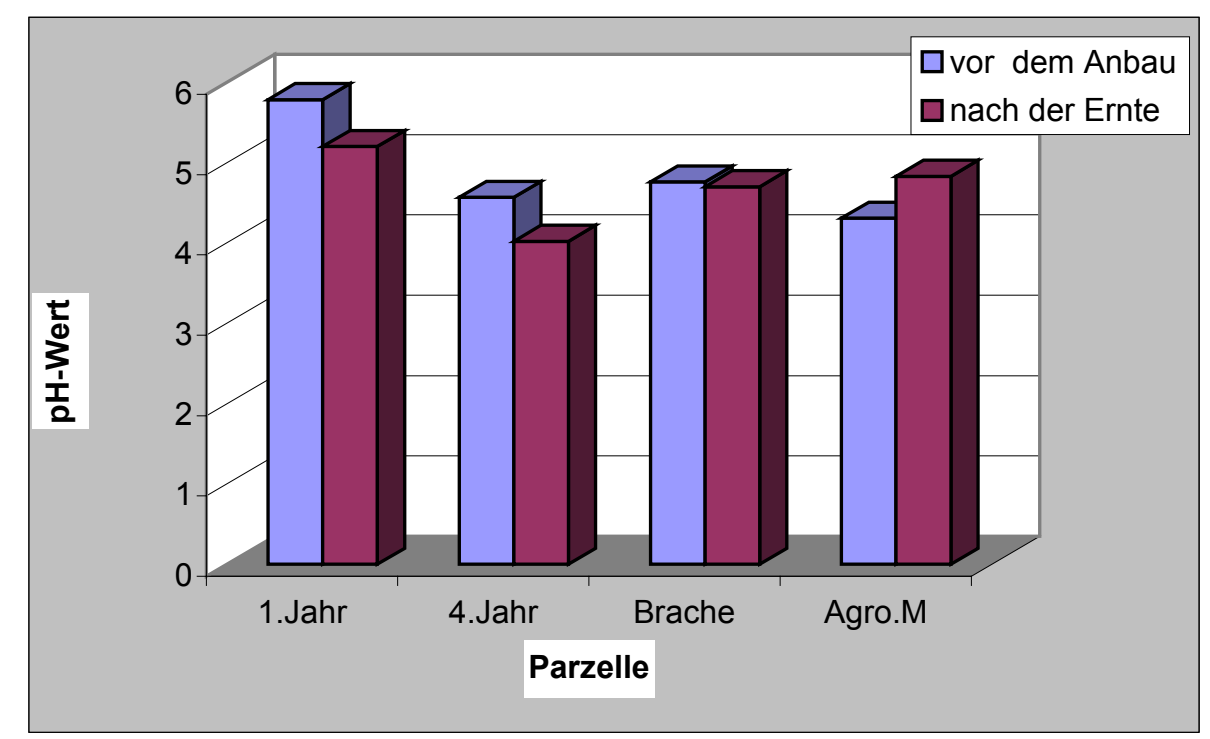

\section{Abb. 26: Die Veränderung der pH-Wert im Oberboden vor dem Anbau und nach der Ernte}

Nach TRAn DuC VIEN (1996) verringern sich die pH -Werte der Böden schnell mit der Dauer der Landnutzung. Der $\mathrm{pH}-$ Wert $(\mathrm{KCl})$ sinkt von $5 \mathrm{im}$ ersten Jahr des Trockenreisanbaus auf 4,1 im 5. Jahr des Anbaus ab. Die Ursache dafür ist der Verlust von Kationen.

Der $\mathrm{pH}-$ Wert ist ein rein chemischer Faktor, der über die Lebensbedingungen von Mikroorganismen auf die Intensität mikrobiologischer Umsetzungen und auf das Anreicherungspotential organischer Substanz (OS) wirkt. Mit sinkendem pH- Wert wechseln die streuzersetzenden Mikroorganismen von Bakterien über Actinomyceten zu Pilzen (AlEXANDER,1980). Weiterhin stehen Boden-pH und Streuqualität in enger Wechselbeziehung zueinander. Eine Abnahme des pHs bedingt eine Abnahme der Zersetzung und eine Reduktion der Mineralisierung. Die Bodenacidität ist für viele Prozesse im Umweltmedium Boden von großer direkter oder indirekter Bedeutung. Die Intensität der chemischen Verwitterung als Bestandteil der Bodenentwicklung nimmt mit sinkendem Boden- $\mathrm{pH} \mathrm{zu}$, die biologische Aktivität und Humifizierung sowie die Verfügbarkeit zahlreicher Pflanzennährstoffe (z.B. S, K, Ca, Mg, Mo) hingehen ab. Andererseits nimmt die Verfügbarkeit bzw. Mobilität vieler Mikronährstoffe (z.B. Fe, 
$\mathrm{Mn}, \mathrm{Cu}, \mathrm{Zn}$ ) oder sonstiger Schwermetalle (z.B. Pb, Cd, Ni) bei annehmendem Boden pH zu. (SCHIEFER, 1984; GOUGH \& MARRS, 1990).

\subsubsection{Organische Substanz und Gesamtstickstoff}

Die organische Substanz spielt bei der Entwicklung und der Funktion terrestrischer Ökosysteme eine tragende Rolle. Die potentielle Produktivität von Ökosystemen steht mit der Menge und dem Umsatz der organischen Bodensubstanz in enger Beziehung. YouNG (1976) schreibt: "Die Bedeutung der organischen Masse in tropischen Böden ist größer als die irgend einer anderen Eigenschaft des Bodens, wenn man einmal von der Feuchte absieht." Und SANCHEZ (1976) betont, dass die Erhaltung der organischen Masse „, von grundlegender Bedeutung für die Produktivität tropischer Böden „, ist.

Betrachtet man die Entwicklung des Gehaltes der organischen Substanz in Anhängigkeit von der Nutzungsdauer, so ist eine deutliche Abnahme der organischen Substanz mit Zunahme der Dauer der traditionellen Wanderfeldbaunutzung zu verzeichnen. Der Oberboden $(0-30 \mathrm{~cm})$ der Waldflächen weist einen hohen Gehalt von 8,1\% auf, nach der Bandrodung weisen die Oberböden nur noch 4,4\% und damit einen mäßigen Gehalt auf. Nach dem 4. Jahr des Maisanbaues nimmt der Humusgehalt stetig und sehr deutlich von 4,4\% auf einen Gehalt von 2,4\% ab. Nach dem 4.Jahr Anbaujahr sind die Böden bereite deutlich degradiert. Die Ursache für die Bodendegradation ist Bodenerosion. (s. Kap. 6.5.2 \& 6.5.3 Bodenerosion und Pflanzenertrag). Deswegen hat der Maisertrag von 3265 $\mathrm{kg} / \mathrm{ha}$ im 1. Jahr auf $962 \mathrm{~kg} / \mathrm{ha}$ im 4. Jahr nach Brandrodung abgenommen. In den traditionellen Landnutzungssystemen des Untersuchungsgebietes lassen die Bauern die Flächen im darauffolgenden Jahr brach liegen zur Rehabilitation der Böden.

Nach Young, (1976) ist die traditionelle Buschbrache ein Mittel, um den Humusstatus der Böden wieder zu verbessern, wobei diese und andere Maßnahmen um so wirksamer sind, je weiter der Boden von seinem optimalen C-Gehalt entfernt ist.

Eine Steigerung des Humusgehalt auf mehr als 75 Prozent des Gehalts unter natürlicher Vegetation kann bei Ackerbau kaum erreicht werden. 


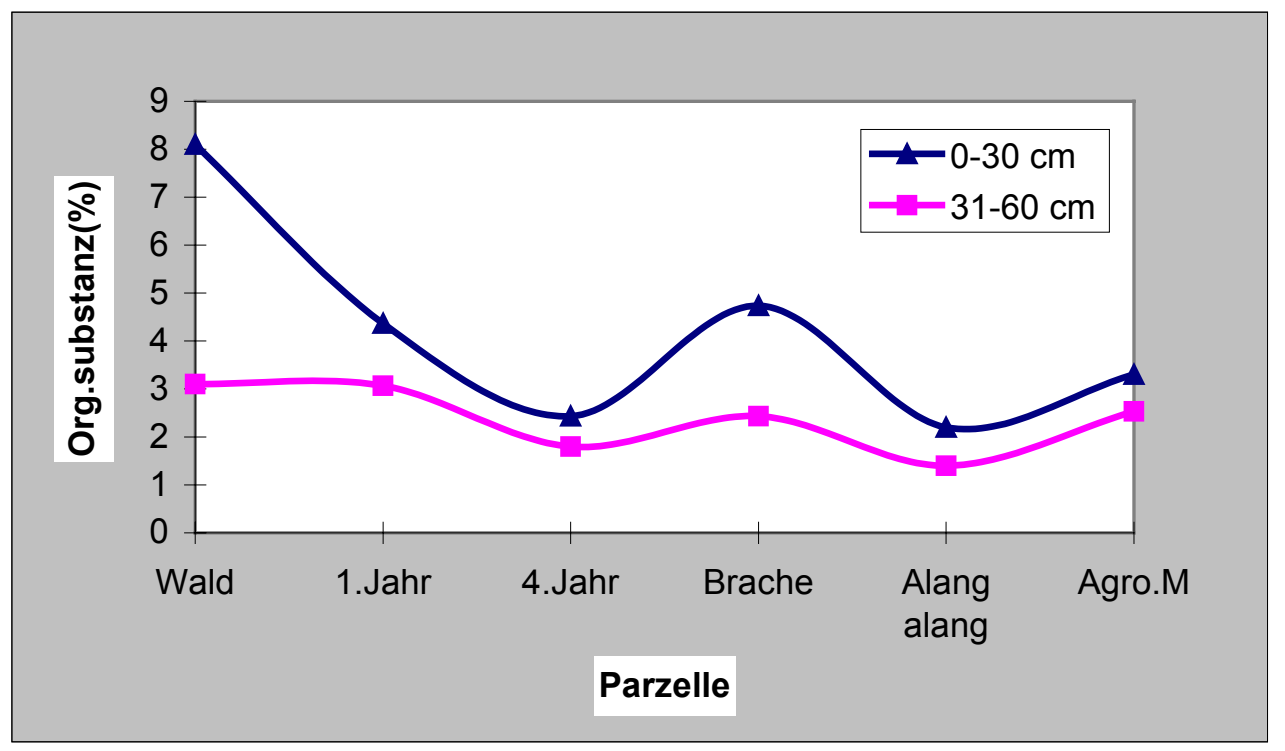

\section{Abb. 27: Die organische Substanz in Abhängigkeit von Anbaudauer und Nutzungstyp}

Darüber hinaus betrachtet man noch die Entwicklung des Gehaltes der organische Substanz im zeitlichen Vergleich, so daß eine deutliche Reduzierung des Gehaltes der organischen Substanz mit Zunahme der Nutzung einhergeht.

Die Fläche des AlangAlang Bewuches weist einen Gehalt von 2,2\% organischer Substanz im Oberboden (0-30 cm) und 1,4\% im Unterboden (30-60) auf. Mit dem 3. Jahre Agroforst mit Mulch nimmt der Humusgehalt von 2,3 auf 3,3\% zu (s. Abb. 27). Die Hecken aus Tephrocia Candida haben eine Fähigkeit, mit ihren Wurzeln eine Symbiose mit Knöllchenbakterien (Rhizobien) einzugehen. Diese sind in der Lage sind, Luftstickstoff zu binden und in organische Form zu überführen. Sie sind deshalb vom Stickstoffangebot des Bodens unabhängig. Außerdem besitzen sie als Gründüngungspflanzen eine gute Langzeitwirkung. Die Pflanzenreste und die Streu von Tephrocia Candida eignen sich besonders als bodenbedeckender Flächenmulch und zur Verbesserung der Bodenstruktur.

Nach BARROW (1991) ist für tropische Böden ein Gehalt an organische Substanz von weniger als 2\% ein Indikator für die Degradation des Bodens.

Parallel zur Entwicklung des C - Gehaltes verringert sich auch der Gesamtstickstoffgehalt der Oberböden in Abhängigkeit von der Nutzungsdauer, wie Abb. 27 deutlich zeigt. Der Stickstoffsgehalt sinkt von 0,38\% im Wald auf 0,12\% im 4. Jahr nach der Brandrodung um über $70 \%$. Dabei findet der größte Teil des N-Abbaus im Oberboden durch die Verbrennung der Biomasse statt. Die Parameter-C und N-verhalten sich im Unterboden 
statisch und ändern sich im Rahmen der Nutzung sehr wenig. Nach dem 4. Jahre Brache hat die Stichstoffgehalt eine Tendenz zur Rehabilitation der Bodenfruchtbarkeit.

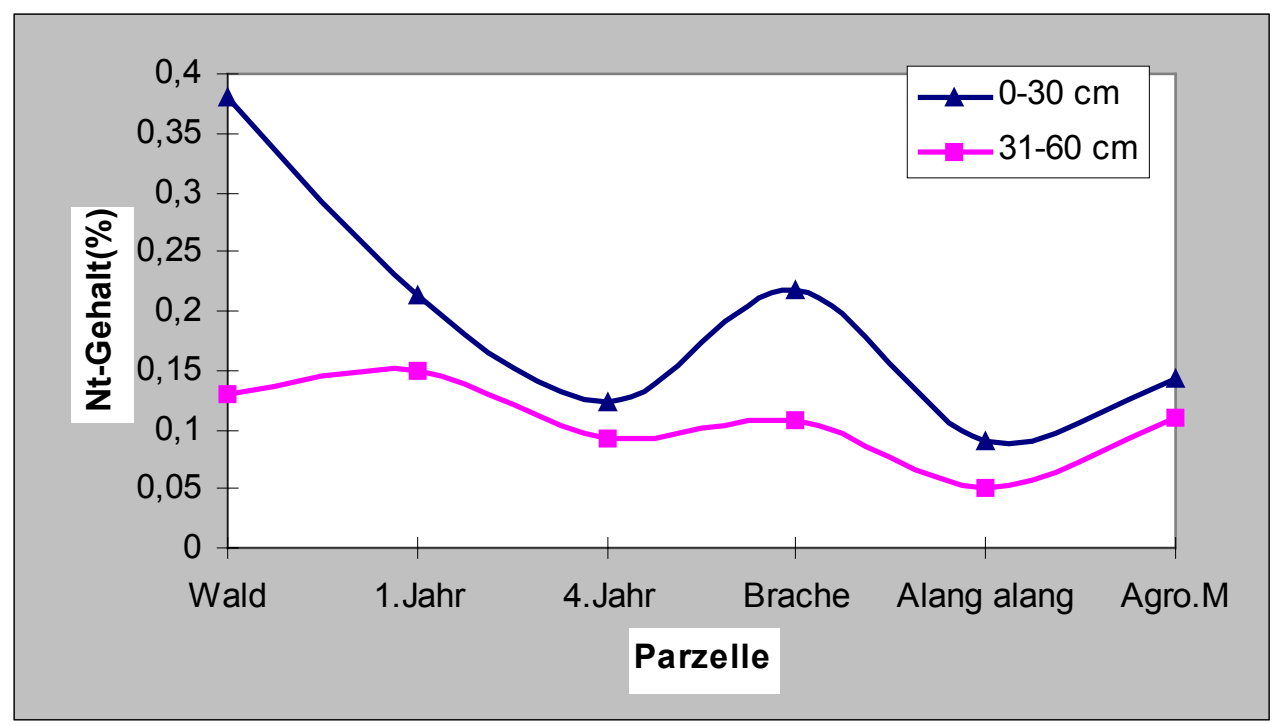

Abb. 28. Gesamtstickstoff in Abhängigkeit von Anbaudauer und Nutzungstyp

Abb.28 stellt noch einmal einen deutlich sinkende Nt- Gehalt im Oberboden (0-30 $\mathrm{cm})$ von $0,38 \%$ auf $0,09 \%$ bei der Fläche Alang alang fest. Aber mit dem Agromulchsystem erreicht der Stickstoffgehalt wieder 0,14\%. Im Unterboden bleibt der Stickstoffgehalt hingegen stabil. Die erklärten Varianzen für organische Substanz und Gesamtstickstoff liegen im Oberboden bei 85 bzw. 86\%. Gemäß dem Bewertungsschlüssel für die Bodennutzung, nach CIAT liegen die Stickstoffgehalte im Oberboden der waldbestandenen Fläche im sehr hohen Bereich, fallen dann nach dem Brand auf hohe Werte ab und darüber hinaus nach 3 Jahren ist die Stickstoff-Versorgung auf den landwirtschaftlichen Flächen nur noch mäßig. Der Unterboden der Fläche ab 31 $\mathrm{cm}$ Tiefe ist vor der Kultivierung noch mäßig mit $\mathrm{N}$ versorgt, mit einsetzender Bewirtschaftung liegen die Stickstoffgehalt nur noch im niedrigen Bereich.

Die beschleunigte Abbau der organischen Substanz nach Eingriffen in Naturwaldsysteme der Tropen zwecks landwirtschaftlicher Bestellung ist vielfach untersucht und belegt worden. WhitMORE (1990) \& TIESSEN ET AL (1992) fanden heraus, dass sich die organische Substanz nach der Brandrodung um 25\% verringert und eine 30\%-ige Verringerung von $\mathrm{C}$ und $\mathrm{N}$ nach 5 Jahren landwirtschaftlicher Nutzung innerhalb eines shifting-cultivation im Nordosten Brasiliens nach sich zieht. In den Böden Vietnams wurde eine KAK von 100 bis 350 mval/100 g organische Substanz ermittelt (PAGEL, 1981). ANDRIESSE \& SCHELHAAS (1987), die die Untersuchung über Nährzyklen im 
Boden benutzt haben, um die Auswirkungen von shifting cultivation unter den klimatischen Bedingungen Sri Lankas, Malaysias und Thailands zu ermitteln, stellten eine 55-58\%- ige Abnahme der organischen Substanz, eine 5-10\% Abnahme von KAK und $>10 \%$ Abnahme der organischen Phosphate nach dem 3. Jahr nach Brandrodung fest. ANDRIESSE \& SCHELHAAS (1987b), die einen Vergleich der Nährstoffgehalte zwischen „Primary forest fallowed by burning ,Sekundärwald und traditionelle kleinbäuerliche Landnutzungssysteme“ erarbeiteten, ermittelten als Hauptursachen für die Verarmung der Nährstoffe in slash-and-burn- system Bodenerosion, Abfluss und Auswaschung, Nährstoffabbau durch geerntetes Getreide, Zerstörung der Waldwurzelmatte und Abnahme der organischen Substanz im Oberflächenboden.
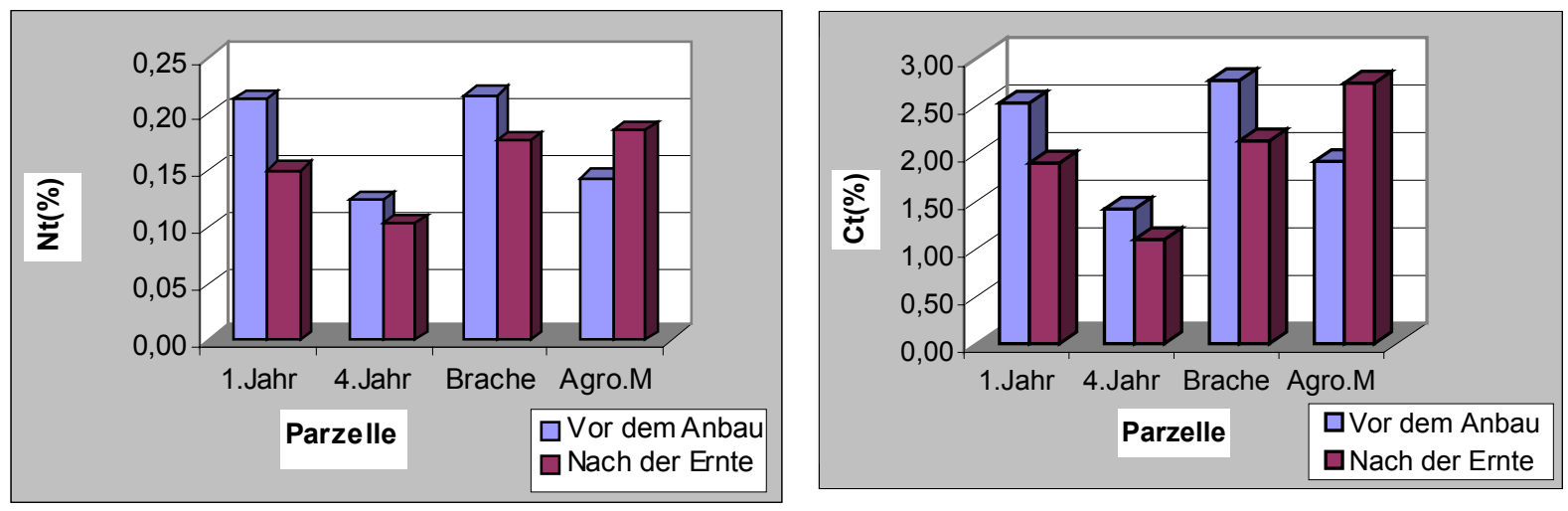

\section{Abb. 29: Die Veränderung von $\mathrm{Ct}$ und Nt vor Anbau und nach der Ernte im Oberboden}

SIEM, 1990 benutzte CI ( Cultivation index = \% Humus der landwirtschaftliche Nutzung/ \% Humus des Waldes), um die Abnahme des Humusgehalts von Böden nach landwirtschaftlicher Bewirtschaftung aufzuzeigen. Im Bergland von Vietnam weisen die Böden eine niedrige $\mathrm{Ct}$ von nur 16-18\% auf. In Indien waren es 30\%, in Südwestamerika 40- 75\% (JENNY \& RAYCHAUDHURI, 1960). Beim Vergleich von 68 Bodenproben errechnete man bei 30 jähriger landwirtschaftlicher Nutzung eine Humusabnahme von 45-60\%, zum Teil sogar bis $80 \%$.

Verursacht wird die Abnahme der organischen Substanz durch den starken Entzug von Biomasse bei der Rodung, Verringerung des organischen Inputs (Ausbleiben des Litterfalls) sowie durch die Zunahme der Dekomposition der organischen Substanz, bedingt durch die erhöhte biologische Aktivität, die durch Bodendurchmischung der Bearbeitung und höhere Bodentemperaturen infolge verstärkter direkter Einstrahlung zustande kommt. (MARKUSSEN, 2000). 
Bei Untersuchungen zur Bodendegradation im Oriente Boliviens zeigte sich beim Vergleich primärer Waldstandorte mit 20 Jahre genutzten Flächen im Mittel ein Rückgang der organischen Substanz von 31\% (GEROLD, 1986b).

Wenn die Böden im Bergand von Vietnam 1\% Humus verloren, stieg die Phosphorfixierung um ca. 50 ppm ( NGUYen Tu SieM \& THAi PHIEN, 1992)

Das $\mathrm{C} / \mathrm{N}-$ Verhältnis des Substrates ist ein wichtiger Faktor zur Bestimmung der Stickstoffmineralisationsrate. Anorganischer N wird freigesetzt, wenn das assimilierte Material mehr $\mathrm{N}$ enthält als die abbauenden Mikroorganismen für ihr Wachstum benötigen. Weisen die Abbauorganismen ein höheres $\mathrm{C} / \mathrm{N}-$ Verhältnis auf als die organische Substanz, wird beim Abbau der organischen Substanz mehr anorganischer Stickstoff freigesetzt. (SCHINNER \& SONNLEITNER, 1996).

Betrachtet man die $\mathrm{C} / \mathrm{N}$-Verhältnisse im zeitlichen Vergleich, so ist eine deutlich erhöhter Werte des C/N- Verhältnisses mit Zunahme der Nutzungsdauer zu verzeichnen. Scheinbar unabhängig von der Bewirtschaftungsform verhält sich das C/N - Verhältnis im Ober-und Unterboden (Abb. 30). Generell zeigen die hohen $\mathrm{C} / \mathrm{N}-$ Werte, dass die organische Fraktion (Humus) des Bodens relativ arm an Stickstoff ist.

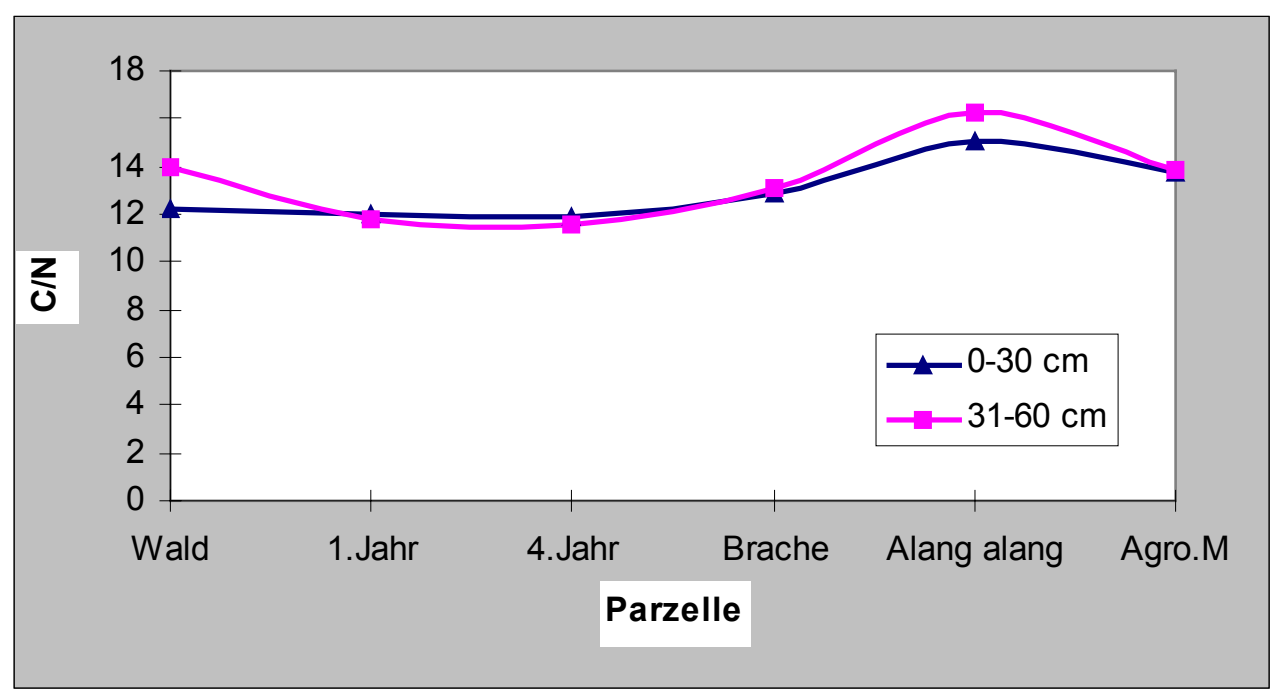

\section{Abb. 30: C/N-Ratio in Ober- und Unterboden in Abhängigkeit von Anbaudauer und Nutzungstyp}

Ein deutliche Zunahme das $\mathrm{C} / \mathrm{N}$ nach langjähriger landwirtschaftlicher Nutzung wird ebenfalls deutlich (s.Abb. 30). Die Waldfläche weist ein C/N -Verhältnis von 12, 2 (Ct: 4,69\% und Nt: 0,38\%) im Oberboden auf. Bei Imperata- Grassland erhöhte sich das C/N auf bis zu 15,1 (Ct: 1,33 \% und Nt: 0,09\%). Sie ist ein Anzeichen für die Degradation der Bodenqualität. Die Abbaurate der organischen Bodensubstanz und Mineralisierungsrate 
von Kohlenstoff und Stickstoff wird durch die Bodentextur und deren Einfluss auf den Porenraum, die Porengrößenverteilung und die Populationen der Mikroorganismen, Protozoen und Nematoden beeinfluss. Nach SCHINNER \& SONNLEITNER (1996) weist die organische Substanz ein C/N- Verhältnis von 15-20 in podsolierten Böden auf. Während in anderen sandigen Böden das C/N- Verhältnis zwischen 10-18 rangiert. In Lehmen und Tonböden betrug das C/N- Verhältnis etwa 10. MöLLER (1979) fand eine verstärkte Humusanreicherung, eine Vergrößerung des C/N- Verhältnisses, die Erhöhung der aktuellen Acidität, eine Verminderung der Konzentration an pflanzenverfügbarem Phosphor und Kalium bei Zugrundelegung gleicher Humusgehalte.

YouNG (1976) zitiert zahlreiche Untersuchungen, wo die Abbaurate des Humus in den ersten 2 bis 3 Jahren bei durchschnittlich 10-20 Prozent lag. Auf Ultisolen im Amazonasbecken betrug der Humusabbau im ersten Jahr nach der Inkulturnahme 25\% (SANCHEZ et al., 1982).

Die Bodennährstoffverarmung auf den Böden der Inneren Tropen bei Brandrodungsfeldbau mit unzureichender Bracheregeneration wird als eine Hauptursache für die Aufgabe der Kolonistenbetriebe mit „Savannisierung“ (z.B. Ausdehnung des Imperata- Graslandes) und Vorrücken der Pionierfront in den Regenwald mit neuer Brandrodung angesehen (GEROLD, 2002).

Der Einfluss, die Funktion und insbesondere die Bedeutung der organischen Substanz auf die Bodenqualität ist wesentlich größer als deren prozentualer Gehalt annehmen ließ. Im Folgenden sind einige wichtige Funktionen der organischen Substanz zusammenstellt: (nach SCHINNER \& SONNLEITNER, 1996; MÜLLER- SÄMANN,1986):

- Funktion als Austauscher bzw. Sorptionskörper.

- Auf den eisenoxidreichen Böden trägt der Umsatz der organischer Substanz zur Mobilisierung von Phosphat bei.

- Förderung der Ausbildung von Bodenaggregaten, prinzipiell fördernde und erhaltende Wirkung auf die Bodenstruktur.

- Verringerung der Plastizität und Kohäsion anorganischer Kornfraktionen.

- Erhöhung der Wasserhaltekapazität. Das Wasseraufnahmevermögen der organischen Substanz kann das Drei- bis Zwanzigfache des Eigengewichtes betragen.

- Speicherung von Nährstoffen in organisch gebundener Form.

- Freisetzung anorganischer Nährstoffe im Zuge der Mineralisierung von organischer Substanz.

- Bildung und Austausch von Nährstoffen, geladenen und ungeladenen Molekülen. 
- Bildung bodenfremder Stoffe (Schadstoffe) wodurch deren biologische Wirksamkeit verringert bzw. deren Persistenz verlängert, sowie deren Mobilität erhöht werden kann.

- Erhöhung der (Mikro) Nährstoffverfügbarkeit durch die Bildung von Chelaten mit mehrwertigen Kationen (z. B . $\mathrm{Cu}^{+2}, \mathrm{Mn}^{+2}, \mathrm{Zn}^{+2}$ ).

- Fördernde Effekte auf Pflanzen bei suboptimalen Wachstumsbedingungen.

- Beeinflussung der Bodenfarbe. Die dunkle Farbe von Huminstoffen kann die Erwärmung der Böden fördern. Da an organischer Substanz reiche Böden auch über hohe Wassergehalte verfügen, sind dunkle Böden nicht immer wärmer als helle.

\subsubsection{Phosphor, Fe und Al}

Sowohl für den Anteil an organischer Substanz als auch für den Gesamtphosphorgehalt ist eine deutliche Abnahme im Verhältnis zur Dauer des traditionellen Wanderfeldbau zu erkennen. Der Gesamtphosphor liegt unter Wald bei $800 \mathrm{mg} / \mathrm{kg}$ Boden und reduziert sich danach sehr stark auf $361 \mathrm{mg} / \mathrm{kg}$ Boden nach dem 4. Jahr nach Brandrodung und unter Maisanbau. Trotz eines sehr geringen Wiederanstiegs während der Brachezeit, sinkt der Gesamtphosphor weiter bis er unter Alang-Alang-Bewuchs nur noch bei $310 \mathrm{mg} \mathrm{P} / \mathrm{kg}$ Boden liegt. Im Unterboden ist dagegen nur eine schwache Änderung des Gesamtphosphorgehaltes sichtbar.

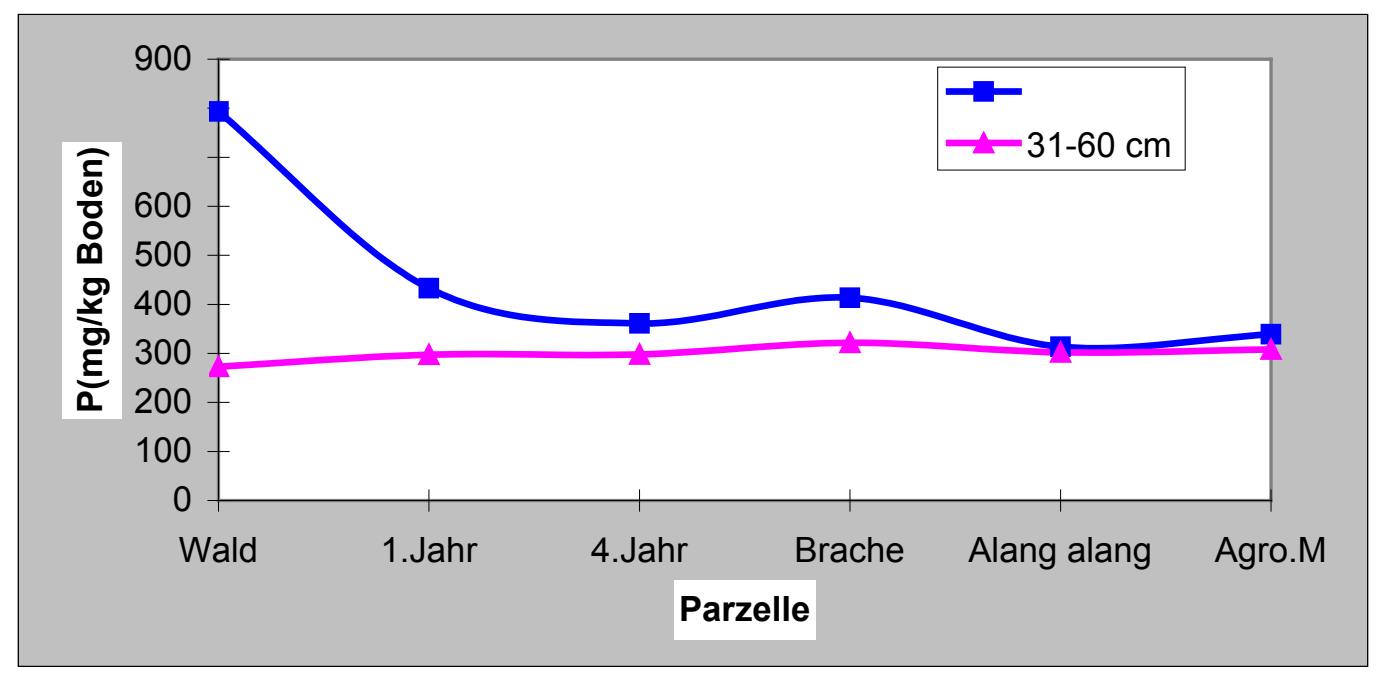

\section{Abb. 31: Gesamt -P-Gehalt in Abhängigkeit von Anbaudauer und Nutzungstyp}

Nach PAGEL (1982) ist die Beurteilung des Gesamt -P-Gehalts eines Bodens schwierig, da die Verfügbarkeit der Phosphate für die Pflanze sehr gering und extrem unterschiedlich sein kann. 
Der Gehalt an pflanzenverfügbarem Phosphor nach Bray (Abb. 32) lässt auf eine sehr schlechte Versorgung der Pflanzen mit Phosphor schließen.

Im Untersuchungsgebiet weisen die Oberböden unter Wald noch Werte um 2,61 mg P $/ \mathrm{kg}$ Boden auf. Nach Brandrodung und Zufuhr von Nährstoffen durch die Asche steigt P-Bray im Oberboden auf den Wert von 3,63 mg/kg Boden (mäßige Versorgung). Trotz eines sehr geringen Wiederanstiegs im 1.Jahr sinkt P-Bray dann weiter, bis er nach dem 4. Jahr unter Maisanbau bei 0,62 mg/kg Boden liegt (83\% des 1.Jahres). Der P-Gehalt ist damit als sehr gering zu beurteilen. Besonders sinkt P-Bray bei Alang-Alang-Bewuchs auf nur noch $0,32 \mathrm{mg} / \mathrm{kg}$ Boden. Alle Werte liegen unterhalb dem niedrigsten Grenzwert von 3 $\mathrm{mg} / \mathrm{kg}$ Boden. Besonders zeigt sich, dass P-pflanzenverfügbar im Brandrodungsfeldbau auf allen Parzellen nach der Ernte stark gesunken ist.

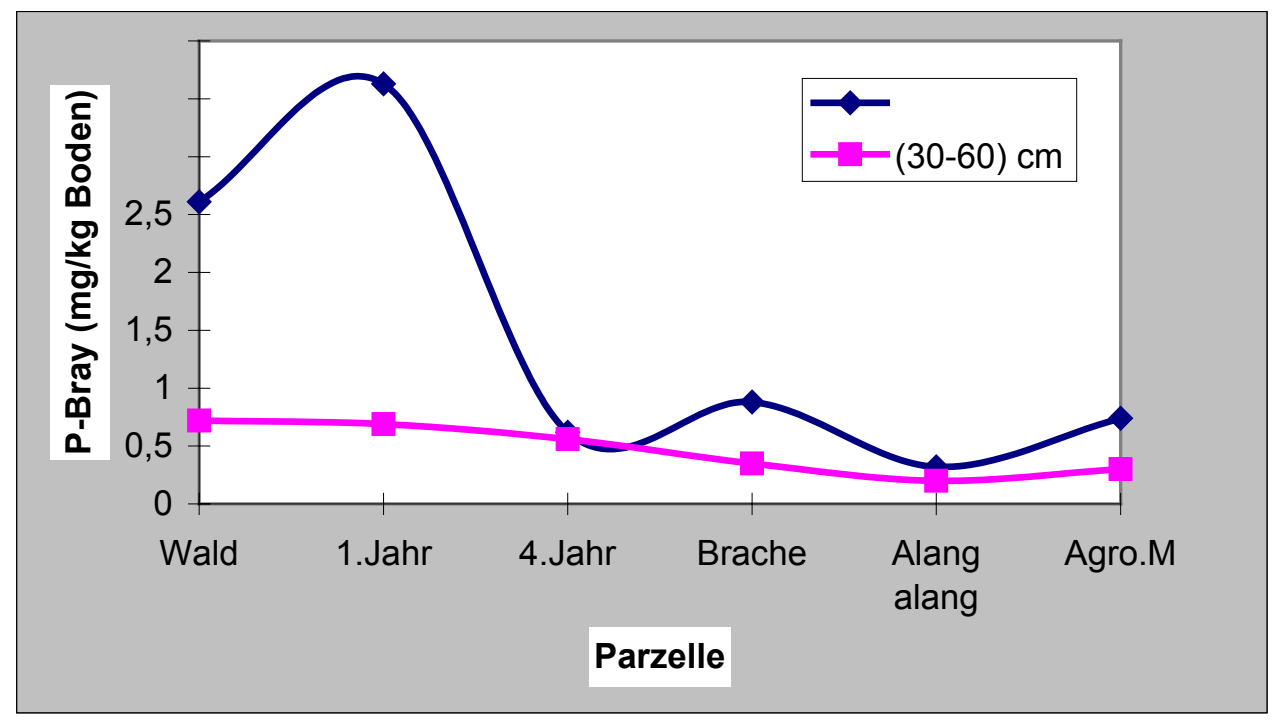

Abb. 32: Die Veränderung von P-pflanzenverfügbar in Abhängigkeit von Anbaudauer und Nutzungstyp

Vergleicht man im traditionellem Anbau P-Bray nach der Ernte mit P-Bray vor dem Anbau so ist ein sehr deutliches Gefälle zur Ernte hin erkennbar. Im Agroforstsystem mit Mulch steigt P-Bray dagegen nach der Ernte von $0,74 \mathrm{mg} / \mathrm{kg}$ Boden auf $0,87 \mathrm{mg} / \mathrm{kg}$ Boden (s.Abb 33).

Eine Abnahme des pflanzenverfügbaren Phosphors bei Andauer der traditionellen Brandrodungswirtschaft liegt u.a. in niedrigen $\mathrm{pH}$-Werten und einer sehr hohen AlSättigung bzw. hohen Al-Konzentration der Böden. Dies führt $\mathrm{zu}$ hoher Phosphatfixierung in den Böden. Desweiteren ist ein schneller Verlust an Bodennährstoffen durch Bodenerosion als Ursache anzusehen. 


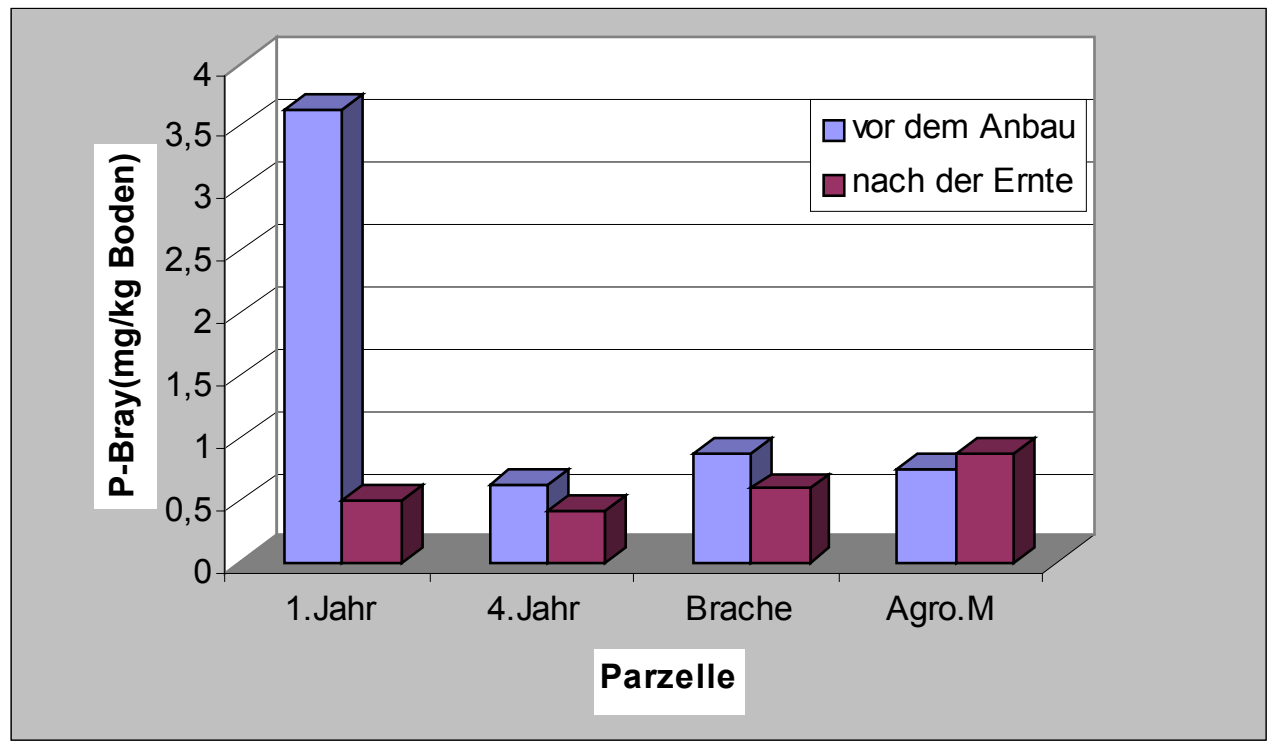

\section{Abb. 33: Die Veränderung von P-pflanzenverfügbar vor dem Anbau und nach der Ernte im Oberboden}

Aufgrund des tropischen Klimas (heiß und feucht) ist das Potential an Phosphor in den Böden Vietnams allgemein sehr niedrig. Je niedriger der pH-Wert des Bodens sowie je höher die Temperatur, je länger die Zeit der Bodentrockenheit, je ärmer der Boden an Humus und je höher der Tongehalt, desto stärker wird Phosphor fixiert. Mit steigender Phosphorfixierung wird auch der pflanzenverfügbare Phosphor fest gebunden, so dass er nicht mehr in den Boden freigesetzt werden kann (SIEM \&T.PHIEN, 1999).

In den sauren Böden wird das P-Ion zuerst durch die Kationen $\mathrm{Fe}^{+3} \& \mathrm{Al}^{+3}$ beeinflusst. Wenn die Böden im Hochland einen $\mathrm{pH}-$ Wert von 4 aufweisen, findet man fast kein $\mathrm{H}_{2} \mathrm{PO}_{4}{ }^{-}$und $\mathrm{HPO}_{4}{ }^{2-}$ mehr. Man kalkuliert für die Böden im vietnamesischem Bergland, dass pro $1 \%$ Verlust an organischer Substanz $500 \mathrm{ppm}$ Phosphor fixiert werden (SIEM \& PHIEN, 1991).

Unter Alang-Alang-Brache lässt sich die geringe Wirksamkeit der eingearbeiteten Biomasse entweder durch ihren relativ geringen Nährstoffgehalt (weites $\mathrm{C} / \mathrm{N}-$ Verhältnis, eventuell biologische P-Blockierung) oder durch eine geringe biologische Aktivität des Bodens (starke Versauerung, Aluminium-Toxizität, Staunässe) erklären.

Böden mit Gehalten von durchschnittlich 4 ppm pflanzenverfügbarem $\mathrm{P}$ sind nach LANDON (1984) als phosphatarm einzustufen. Bei HofFMANN (1991) wird der Boden schon bei Durchschnittsgehalten kleiner 3 ppm P-pflanzenverfügbar als phosphatarm eingestuft.

Die Wirkung des Verbrennens von Biomasse in Shifting cultivation auf die Phosphorverwendbarkeit wird in der folgenden Abbildung analysiert. 


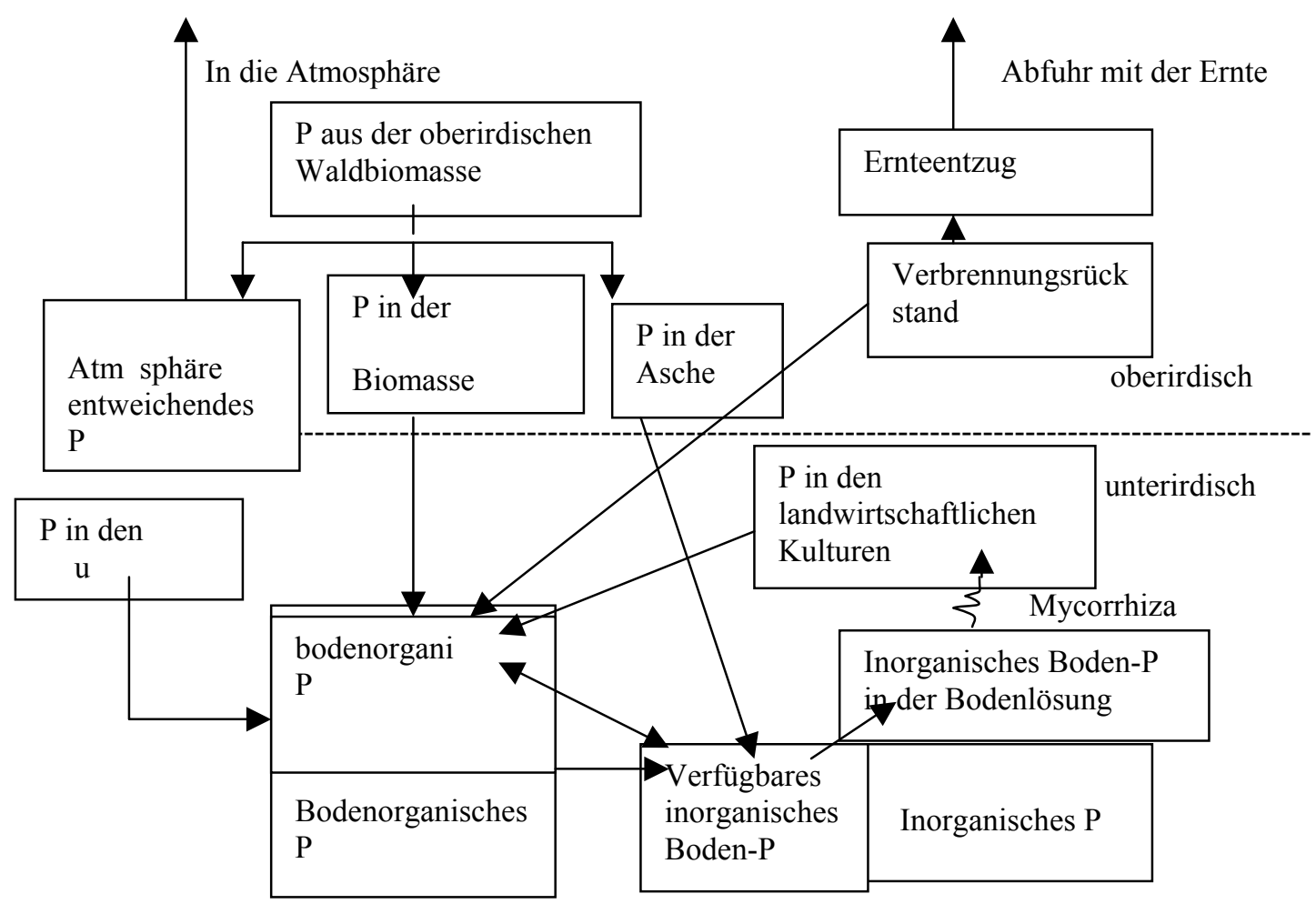

Abb. 34: Der Phosphorkreislauf während der Wachstumsperiode in shifting cultivation

(vereinfacht aus Sanchez et al., 1991). Der inorganische P-Pool beinhaltet nichtverfügbar fixiertes und okkluiertes $P$ sowie verwitterbares $P$, welches freigesetzt dem verfügbaren inorganischem $P$ zufällt.

In den Untersuchungen von JORDAN (1989) wird betont, dass für die Tropen ein charakteristischer Ertragsrückgang mit der Nutzungsdauer aus steigender Aluminiumtoxizität, Eisenoxid- und Phosphatfixierung resultiert, die nur wenige Nutzpflanzen tolerieren. Ergebnisse aus den Provinzen Vinh Phu und Hoabinh in Vietnam zeigen, dass organischer Kohlenstoff, pflanzenverfügbares Phosphor und Basensättigungsgrad in kultivierten Feldböden (Shifting cultivation) unterschiedlichen Alters (1-3 Jahre) viel niedriger sind als in vergleichbaren Waldböden.

In sauren Böden tritt $\mathrm{P}$ als $\mathrm{Fe}^{+3}$ - und Al-Phosphat sowie in Form von „Oberflächenphosphaten“ auf, im neutralen Bereich als Ca- Phosphat. Die Verlagerung von gelöstem P ist im Mineralboden sehr gering, da er leicht durch Fällung oder Sorption immobilisiert wird. Entsprechend gering ist der P-Austrag in gelöster Form (wenige g P $\left.\mathrm{ha}^{-1} \mathrm{a}^{-1}\right)$.

Da die Bodenacidität ebenfalls die Phosphatreaktion mit Eisen, Aluminium und Mangan beeinflusst, ist der Anteil des pflanzenverfügbaren (löslichen) Phosphats als bedeutender 
Nährstofffaktor für die Pflanzen, vom pH-Wert anhängig (JORDAN, 1989). Entsprechend der eben herausgestellten Korrelation wurde im Untersuchungsgebiet aufgrund der stark sauren Bodenbedingungen ein sehr niedriger Gehalt an pflanzenverfügbarem Phosphor festgestellt (s. Abb.35).

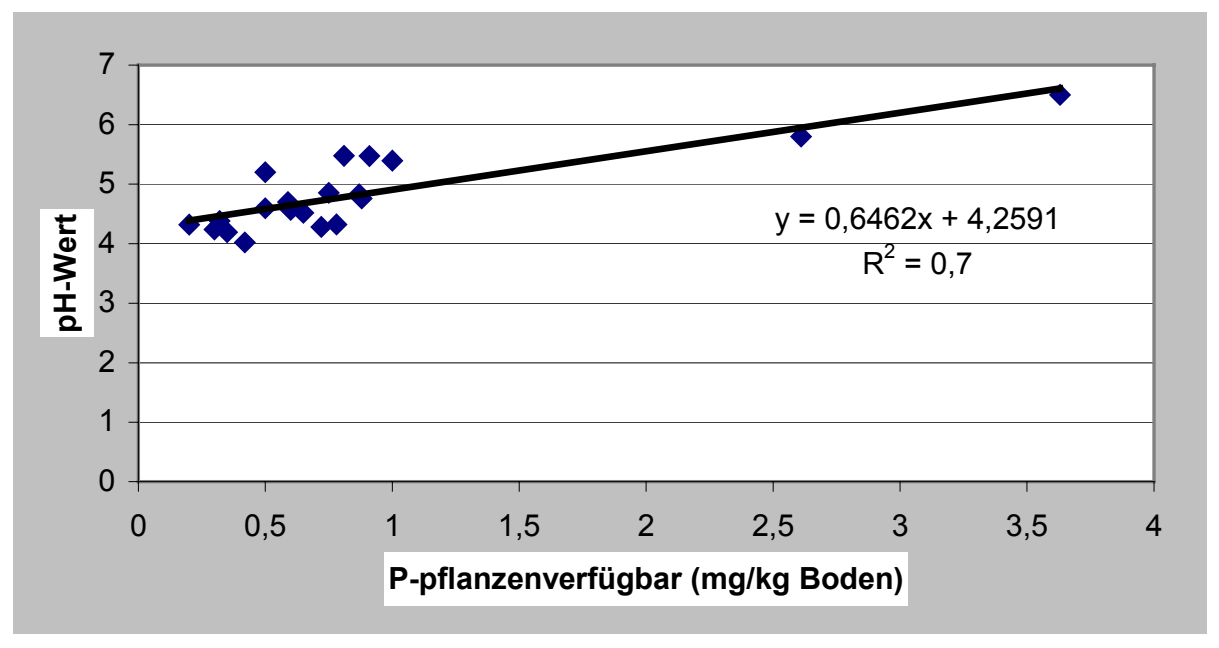

\section{Abb. 35: Korrelation zwischen pH und P- pflanzenverfügbar}

Hohe Gehalte an Eisenoxiden bewirken eine meist gute Bodenstruktur und eine gute Wasserführung. Zum Teil kommt es aber zu Eisentoxizität, speziell bei staunassen Bedingungen. Die Toxizität wird durch reduziertes Eisen $\left(\mathrm{Fe}^{+2}\right)$ hervorgerufen, das bei niederen $\mathrm{pH}$ - Werten und niederem Redoxpotential aus den Fe- Oxiden in Lösung geht.

Dass $\mathrm{Fe}^{+2}-$ Bildung auch schon bei relativ neutralen $\mathrm{pH}-$ Verhältnissen möglich ist, wird in der folgenden Gleichung (nach SCHEFFER \& SCHACHTSCHABEL, 1982) dargestellt:

$$
\mathrm{Fe}(\mathrm{OH})_{3}+3 \mathrm{H}^{+}+\mathrm{e}^{-}=\mathrm{Fe}^{2+}+3 \mathrm{H}_{2} \mathrm{O}
$$

Das bedeutet: Je saurer ein Boden und je mehr Luftmangel im Boden herrscht, desto eher ist mit Eisentoxizität $\mathrm{zu}$ rechnen (z. B nach Einarbeitung unzersetzter organischer Substanz unter staunassen Verhältnissen). Bereits humifizierte organische Substanz (z.B. Kompost) wirkt auch hier durch Bildung größerer Komplexe der Eisentoxizität entgegen. In der Regel ist es jedoch nicht die Toxizität von Eisen, welche das Wachstum von Pflanzen limitiert, sondern die Eigenschaft der Eisenoxide, Phosphat zu fixieren. Phosphatmangel (bzw. Phosphat- Adsorption ) ist besonders ausgeprägt in den tropischen Verwitterungsböden mit hohen Eisenoxidgehalten.

Die spezifische P- Sorption an Eisen- und Al- Oxide (s. Abb. 36) ist relativ stabil, so dass solches Phosphat für Pflanzen praktisch nicht verfügbar ist. 
$(\mathrm{Al}, \mathrm{Fe})-\mathrm{OH}_{2}{ }^{+}+\mathrm{H}_{2} \mathrm{PO}_{4}{ }^{-}=(\mathrm{Al}, \mathrm{Fe})-0-\mathrm{PO}(\mathrm{OH})_{2}+\mathrm{H}_{2} \mathrm{O}$

$\mathrm{O}\left\langle\underset{(\mathrm{Al}, \mathrm{Fe})-\mathrm{OH}}{(\mathrm{Al}, \mathrm{Fe})-\mathrm{OH}}+\mathrm{H}_{2} \mathrm{P}_{4}^{-}=\mathrm{O}<_{(\mathrm{Al}, \mathrm{Fe})-\mathrm{O}}^{(\mathrm{Al}, \mathrm{Fe})-\mathrm{O}}>\mathrm{P}\right\rangle_{\mathrm{OH}}+\mathrm{O}+\mathrm{OH}^{-}+\mathrm{H}_{2} \mathrm{O}$

\section{Abb.36: Spezifische Adsorption von Phosphat an Eisen- oder Aluminiumoxide (aus SCHEFFER \& SCHACHTSCHABEL, 1982)}

Mit steigendem $\mathrm{pH}-$ Wert sinkt die Phosphatadsorption, da Hydroxydionen mit Phosphat um die Sorptionsplätze konkurrieren.

Die Phosphatverfügbarkeit in den tropischen Böden hängt aber nicht nur von Gehalt an Eisen- Aluminiumoxiden ab, sondern wird auch stark vom Gehalt der Böden an organischer Masse und im Fall von Andosolen von deren Allophangehalt beeinflusst.

Ähnlich wie Phosphat kann auch Molybdat (geringer Sulfat und Borat) gebunden, d.h. spezifisch sorbiert werden.

Die unspezifische Adsorption von Anionen (sie erfolgt ausschließlich an variablen Ladungen) ist $\mathrm{pH}$-abhängig und findet in den Fe-Al-oxidreichen, kaolinitischen Böden schon im mäßig sauren $\mathrm{pH}-$ Bereich statt (bei pH 6). Die oxidreichen Böden wirken dann fast als reine Anionenaustauscher und sorbieren bei $\mathrm{pH}-$ Werten unterhalb des Ladungsnullpunktes z.B. $\mathrm{NO}_{3}{ }^{-}$und $\mathrm{Cl}^{-}$, aber kaum Kationen. Bei Anwesenheit von viel organischer Substanz (Ladungsnullpunkt im stark sauren Bereich) und bei steigendem pH-wert geht die Anionenadsorption zurück, die Kationensorption wird deutlich besser.

Bei $\mathrm{pH}<4$ macht $\mathrm{Al}^{+3}$ etwa $80 \%$ der Austauscherbelegung aus. In stark sauren Böden kann der gemeinsame Anteil von $\mathrm{H}$ und $\mathrm{Al}$ über 90\% erreichen. Unterhalb von $\mathrm{pH} 4,5$ ist $\mathrm{Al}^{+3}$ die dominierende Ionenspezies im Mineralboden. Oberhalb $\mathrm{pH}$ 5,5 ist die AlSättigung des Sorptionskomplexes gering. Steigt die Al- Sättigung des Sorptionskomplexes auf $>60 \%$, so geht $\mathrm{Al}$ zunehmend in die Bodenlösung über. Bei pH 5 ist die vorherrschende Al-Verbindung in der Lösungsphase $\mathrm{Al}(\mathrm{OH})_{3}$, bei $\mathrm{pH} 4,2$ kann mit gleichen Anteilen von $\mathrm{Al}(\mathrm{OH})_{3}, \mathrm{Al}(\mathrm{OH})_{2}{ }^{+}$und $\mathrm{Al}^{3}+$ gerechnet werden (FIEDLER, 2001)

Betracht man die Entwicklung des Gehaltes von Fe, Al (s. Abb.37 \& 38) im zeitlichen Vergleich, so ist ein deutliche Zunahme des Gehaltes von Fe, Al mit zunehmender Dauer des traditionellemWanderfeldbaus zu verzeichnen. Dagegen entwickelt sich der PGehalt im umgekehrtem Sinn. Er sinkt mit anhaltendem Anbau. Auf der Parzelle Agroforst mit Mulch weist der Al, Fe-Gehalt eine abnehmende Tendenz auf. 


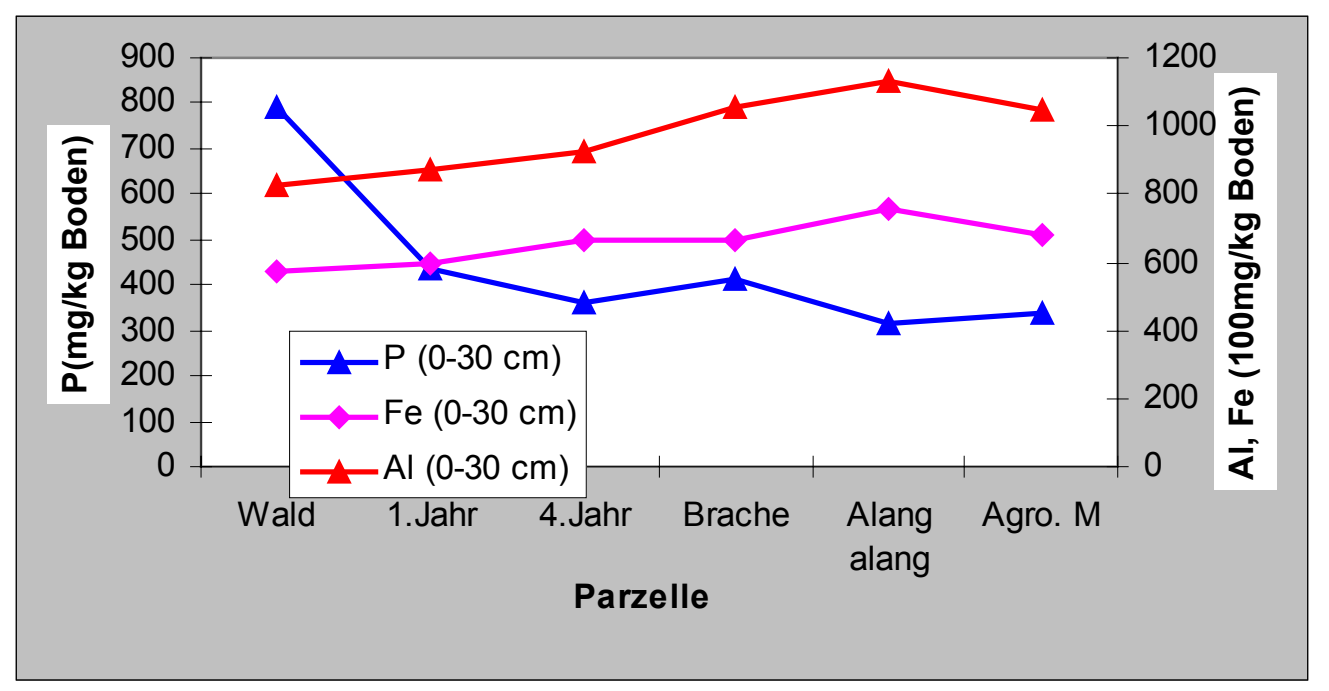

Abb. 37: Veränderung von $\mathrm{Al}, \mathrm{Fe}$ und $\mathrm{P}$ in einem Zyklus des traditionellen Wanderfeldbaues, Alang Alang Brache und Agroforst mit Mulch

Intensiver Wassertransport führt $\mathrm{zu}$ Bodenversauerung, sowie zur Freisetzung von Aluminium, Eisen und Mangan in niedrigeren Pufferbereichen des Bodens. Die Eisenund Aluminiumanreicherung in niedrigeren $\mathrm{pH}$ - Bereichen bewirkt eine verringerte Phosphorverfügbarkeit durch irreversible Phosphatfixierung. Das natürliche Regenerationsvermögen der Böden wird herabgesetzt.

Der Rückgang der Bodenfruchtbarkeit erfolgt in zeitlicher Abhängigkeit von seinen Bodeneigenschaften (Humus-, Nährstoff- und Tongehalt, pH-Wert, Aggegatstabilität, Mikroorganismen) sowie von anthropogenen Einflüssen durch Fruchtfolge, Nutzungsintensität und bodenschützende Maßnahmen. Zur Regeneration der Bodenfruchtbarkeit ist eine Bracheperiode erforderlich.

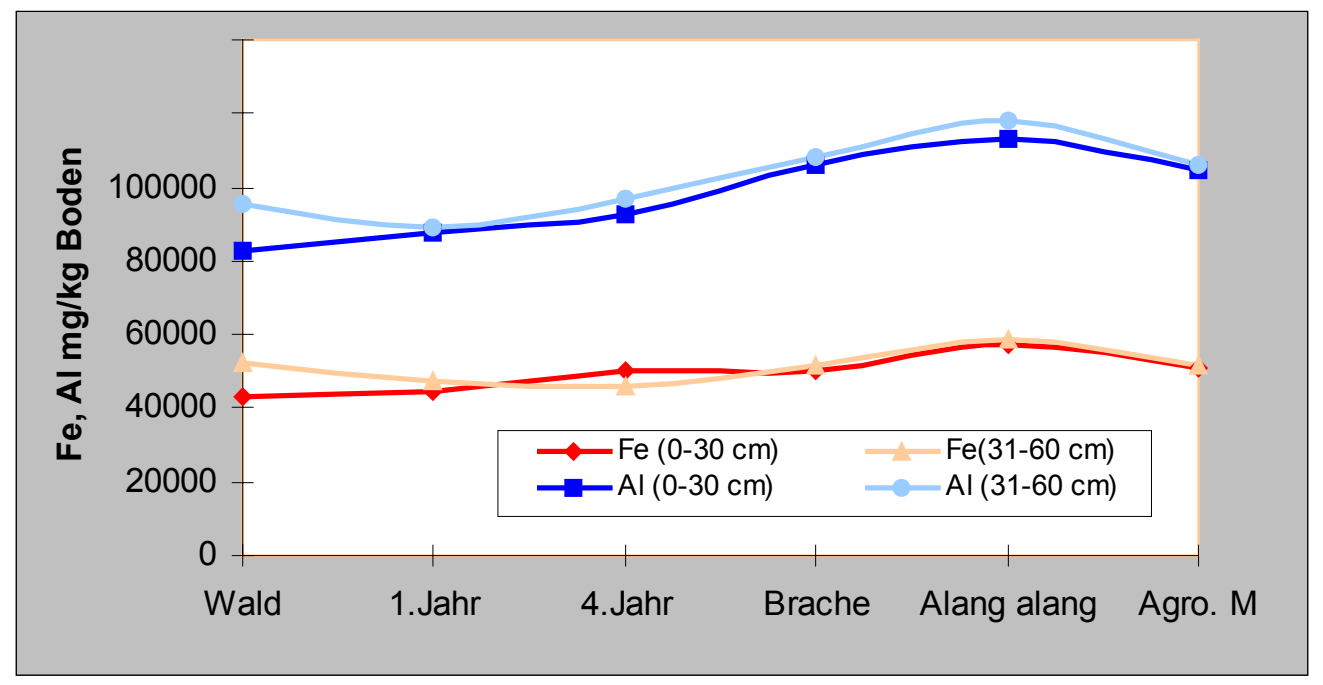

Abb. 38: Veränderung von $\mathrm{Fe}$ und $\mathrm{Al}$ in einem Zyklus des traditionellen Wanderfeldbaues, Alang-Alang-Brache und Agroforst mit Mulch 
Im traditionellem Wanderfeldbau steigen die Al-, Fe-Gehalte nach der Ernte deutlich an (s. Abb. 39). Die Parzelle Agroforst mit Mulch weist dagegen eine abnehmende Tendenz beider Gehalte auf.
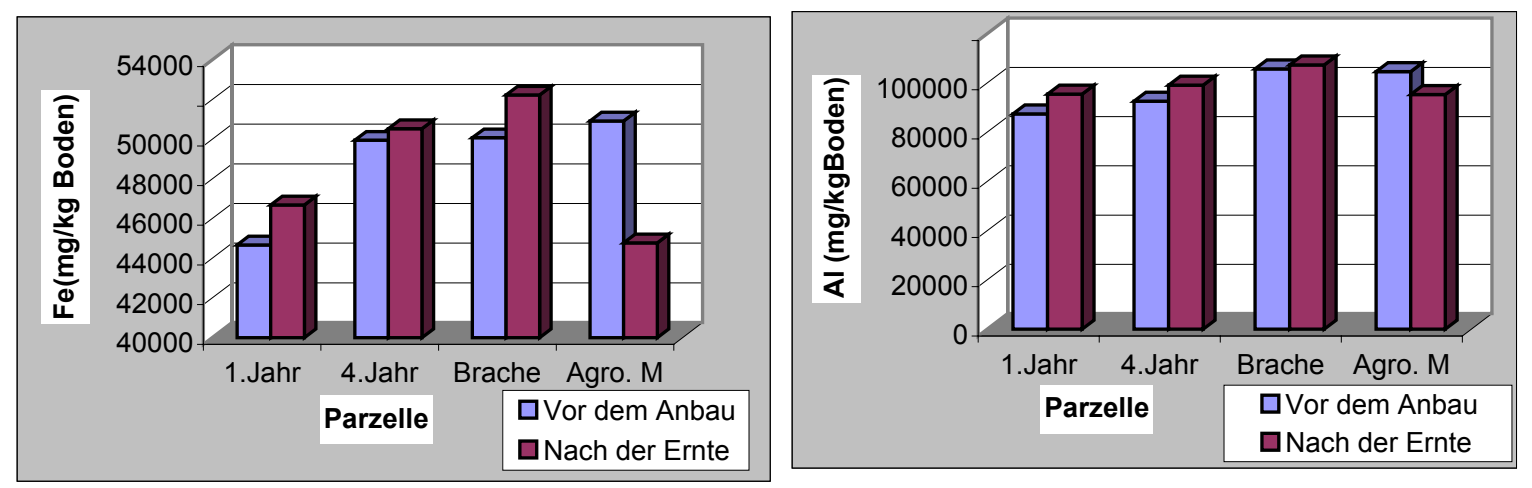

\section{Abb. 39: Fe, Al vor dem Anbau und nach der Ernte im Oberboden}

Diese Ergebnisse entsprechen den Untersuchungen von BUI (1990). Sie stellten im Oberhangboden fest, dass der pH-Wert saurer und der Boden ärmer an Phosphor ist als im Mittelhangboden und Unterhangboden. Im Laufe der Zeit des Wanderfeldbaus nimmt die Bodenfruchtbarkeit deutlich ab: z.B der Humusgehalt betrug im 3.Jahr nur 50\% im Vergleich zum 1.Jahr nach Brandrodung. Im 1. Jahr wurde fast kein $\mathrm{Al}^{+3}$ festgestellt, dass bis zum 3.Jahr aber auf 30-40 $\mathrm{mg} \mathrm{Al}^{+3} / 100 \mathrm{~g}$ Boden ansteigt.

Auf Böden mit pH- Werten unter 5 sind die Austauch- oder Sorptionskörper nicht nur mit Wasserstoff- und Nährstoffkationen ( $\mathrm{Mg}, \mathrm{K}, \mathrm{Ca}$ ) besetzt, sondern sehr häufig zu mehr als 50 Prozent mit Aluminium (Al)-Ionen belegt, was leicht zu Aluminiumtoxizität und zur Phosphatfixierung durch Bildung von Al- Phosphaten führt. Nach SANCHEZ ( 1976) stellt die Azidität (hohe Al-Sättigung bzw. hohe Al-Konzentration, niederer pH, Gefahr von Mangan- Toxizität usw.) ein Hauptproblem vieler tropischer Böden dar. Erst ab pH Werten über 5 fällt Aluminium als Hydroxid aus, so daß Toxizität nicht mehr zu befürchten ist. (Al- Toxizität äussert sich in vermindertem, gestauchtem Wurzelwachstum; (der Ca- und P- Stoffwechsel ist häufig gestört).

\subsubsection{Textur}

In Tab. 25 ist die Textur der verschiedenen Parzellen dargestellt. Die Böden im Untersuchungsgebiet weisen aufgrund starker Verwitterungsprozesse einen sehr hohen Anteil an Feinsubstanz mit 80\% - 90\% auf. Es handelt sich dabei im allgemeinem um 
schluffig-tonigen Lehm bis schluffigen Ton, was auch bei den Felduntersuchungen festgestellt wurde. Der Tonanteil schwankt zwischen 30 bis 50\% mit Ausnahme des Oberbodens unter Brache (6,68\%).

Zum Unterboden hin ist außer bei Alang Alang eine mehr oder weniger deutliche Zunahme des Tongehaltes festzustellen, was durch die hohe chemische Verwitterung verbunden mit geringeren $\mathrm{pH}-$ Werten im Unterboden bedingt ist. Unter Wald, wo der $\mathrm{pH}$ Wert am deutlichsten vom Oberboden zum Unterboden hin abnimmt, ist auch der größte Sprung im Tongehalt von oben nach unten erkennbar.

Tab. 25: Textur in Abhängigkeit von der Dauer der traditionale Wanderfeldbau

\begin{tabular}{|c|c|c|c|c|c|c|c|c|c|}
\hline \multirow{2}{*}{ Parzell } & \multirow{2}{*}{$\begin{array}{l}\text { Boden- } \\
\text { tiefe }\end{array}$} & \multicolumn{3}{|c|}{ Sand $[\mu \mathrm{m}]$} & \multicolumn{3}{|c|}{ Schluff $[\mu \mathrm{m}]$} & \multirow{2}{*}{$\begin{array}{c}\begin{array}{c}\text { Ton } \\
{[\mu \mathrm{m}]}\end{array} \\
0-2 \\
(\%)\end{array}$} & \multirow{2}{*}{$\begin{array}{c}\text { AG } \\
\text { Boden }\end{array}$} \\
\hline & & $\begin{array}{c}630- \\
2000(\%)\end{array}$ & $\begin{array}{c}200- \\
630(\%)\end{array}$ & $\begin{array}{c}63- \\
200(\%)\end{array}$ & $\begin{array}{c}20-63 \\
(\%)\end{array}$ & $\begin{array}{c}6.3-20 \\
(\%)\end{array}$ & $\begin{array}{c}2-6.3 \\
(\%)\end{array}$ & & \\
\hline \multirow[t]{2}{*}{ Wald } & $0-30 \mathrm{~cm}$ & 1,97 & 6,04 & 13,12 & 18,47 & 12,37 & 8,69 & 39,35 & Lt3 \\
\hline & $31-60 \mathrm{~cm}$ & 0,49 & 2,08 & 9,61 & 15,48 & 13,03 & 7,91 & 51,40 & Tu2 \\
\hline \multirow[t]{2}{*}{ 1.Jahr } & $0-30 \mathrm{~cm}$ & 0,40 & 1,58 & 7,17 & 22,93 & 24,43 & 8,70 & 34,82 & Tu3 \\
\hline & $31-60 \mathrm{~cm}$ & 0,35 & 1,68 & 7,03 & 22,70 & 22,35 & 8,28 & 37,63 & Tu3 \\
\hline \multirow[t]{2}{*}{ 4. Jahr } & $0-30 \mathrm{~cm}$ & 0,30 & 3,20 & 10,60 & 25,60 & 21,00 & 8,00 & 31,30 & Tu3 \\
\hline & $31-60 \mathrm{~cm}$ & 0,57 & 2,32 & 9,36 & 23,28 & 21,02 & 8,17 & 35,28 & Tu3 \\
\hline \multirow[t]{2}{*}{ Brache } & $0-30 \mathrm{~cm}$ & 0,47 & 2,63 & 6,45 & 13,79 & 27,51 & 42,47 & 6,68 & Us \\
\hline & $31-60 \mathrm{~cm}$ & 1,12 & 2,32 & 5,76 & 19,00 & 24,22 & 8,56 & 39,02 & Tu3 \\
\hline \multirow{2}{*}{$\begin{array}{l}\text { Alang } \\
\text { Alang }\end{array}$} & $0-30 \mathrm{~cm}$ & 0,28 & 1,42 & 7,92 & 15,69 & 15,53 & 7,07 & 52,09 & Tu2 \\
\hline & $31-60 \mathrm{~cm}$ & 2,89 & 5,68 & 10,50 & 20,40 & 13,00 & 8,38 & 39,16 & $\mathrm{Lt} 3$ \\
\hline \multirow{2}{*}{$\begin{array}{c}\text { Agro. } \\
\text { M }\end{array}$} & $0-30 \mathrm{~cm}$ & 0,21 & 1,32 & 9,24 & 17,49 & 15,48 & 8,31 & 47,95 & Tu2 \\
\hline & $31-60 \mathrm{~cm}$ & 1,04 & 1,26 & 7,81 & 16,25 & 14,04 & 9,77 & 49,83 & $\mathrm{Tu} 2$ \\
\hline
\end{tabular}

Unter Agroforstnutzung mit Mulch 3 Jahre Nutzung beträgt der Tongehalt fast 50\%. Es handelt sich dabei um den Versuch der Rehabilitierung einer ehemaligen Brachefläche mit Alang Alang. Alang-Alang-Bestand stellt sich auf Flächen mit ausgesprochen niedrigem $\mathrm{pH}-\mathrm{Wert}$ ein.

Ein hoher Anteil an Feinsubstanz im Bodensubstrat wirkt sich allgemein negativ auf die Erodibilität der Böden aus. Hoher Tongehalt fördert den natürlichen Prozess der Kompaktierung (,hardsetting“) der Böden (LAL ET AL., 1989 sowie BARBER, 1995). 
Andererseits können damit niedrige Infiltrationsraten verbunden sein. Hohe Sesquioxidgehalte $(\mathrm{Fe}, \mathrm{Al})$ führen in den Ferralsols jedoch zu einem grobporenreichen Gefüge mit guter Wasserleitfähigkeit (s. Tab. 25, Oberflächenabfluss ab $0,15 \mathrm{~mm} / \mathrm{min}$ Niederschlagsintensität).

\subsubsection{Kationenaustauschkapazität}

Die Kenntnis der austauschbaren Kationen ist nicht nur eine Voraussetzung zur Beurteilung der Bodenfruchtbarkeit, sondern auch zur Abschätzung der Nährstoffversorgung, des Düngerbedarfs und der möglichen Düngerwirkung. Die KAK gilt als Maß für die durch Sorption festgehaltenen Nährstoffe, die so vor Auswaschung geschützt werden. Zu Nährstoffeinträgen bzw. Freisetzungen kommt es durch Niederschläge, Verwitterungs- und Mineralisationsprozesse.

Die KAK liegt in den Ah- Horizonten mit den höchsten Humusgehalten entsprechend höher als in den Unterbodenhorizonten. Dabei wird deutlich, dass der Humusanteil in den Tropen eine wesentlich bedeutsamere Rolle spielt, als die Tonfraktion, wenn diese hauptsächlich aus dem sorptionsschwachen Zweischichtmineral Kaolinit besteht (PAGEL 1982, MÜLlER-SÄMAnN 1986, SchefFer/SchachtSchabel, 1992). Die Betrachtung der KAKeff in Abb. 40 zeigt eine deutliche Differenzierung der Nutzungsart. Im Wald weisen die Oberböden noch Werte um $28 \mathrm{cmol} / \mathrm{kg}$ (hoch) auf. Nach dem 1. Jahr Brandund trotz Zufuhr von Nährstoffen durch die Asche- sinkt die KAKeff im Oberboden auf den Wert 10,7 cmol/kg Boden (gering), was einer Abnahme von 62\% entspricht.

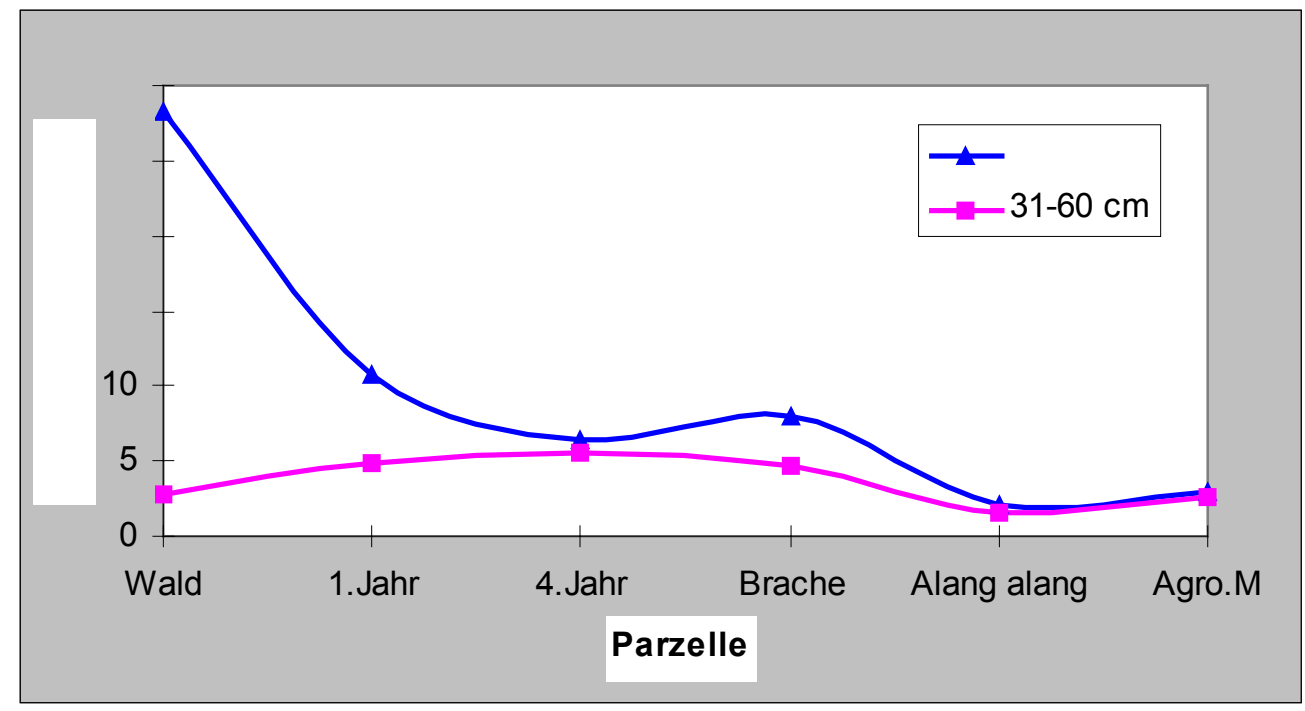

Abb. 40: KAKeff in Abhängigkeit von Nutzungsdauer und Nutzungstyp 
Im 4.Jahr sinkt die KAKeff weiter auf nur noch $6,3 \mathrm{cmol} / \mathrm{kg}$ Boden (niedrig), was $42 \%$ des 1.Jahres entspricht. Die Werte der KAKeff im Unterboden nehmen auf der Parzelle mit Alang Alang weiter ab und sind mit 2,1 cmol/ $\mathrm{kg}$ ebenfalls als niedrig einzustufen. Aus diesen Ergebnissen lässt sich schlußfolgern, dass der Oberboden im Untersuchungsgebiet sehr schnell degradiert. Durch die Anlage von Hecken aus Tephrocia candida und Leuceana glauca versucht man den Nährstoffgehalt der Flächen mit Alang-Alang-Bewuchs zu verbessern.

Die Abnahme der organischen Substanz als Träger von austauschbaren Nährstoffen wirkt sich auf die gesamte Austauschkapazität aus. $\mathrm{Zu}$ den wichtigsten Kationenaustauschern gehört der Gehalt des Boden an Huminstoffen (s. Abb. 20 Korrelation von KAKeff mit organischer Sustanz). Da die organische Substanz innerhalb der falschen Zeitreihe sehr stark zurückgegangen ist, erklärt sich damit auch der deutliche Gradient der KAKeff in Abhängigkeit von der Dauer der landwirtschaftlichen Nutzung.

FIEDLER (2001) schreibt: „Im humusreicheren Oberboden steigt die KAKeff gegenüber dem humusarmen Unterboden an, in besseren Waldböden z.B. von 50-60 auf 150-170 umol $_{\mathrm{c}} \mathrm{g}^{-1}$. Sie liegt damit in der Größenordnung von 10-15 $\mathrm{kmol} \mathrm{ha}^{-1} \mathrm{~cm}^{-1 “}$.

Die Kationenaustauschkapazität der organischen Bodensubstanz nimmt mit steigendem pH zu. In Böden der feuchten Tropen ist bei Fehlen von Dreischicht-Tonmineralen die KAKeff allgemein sehr gering.

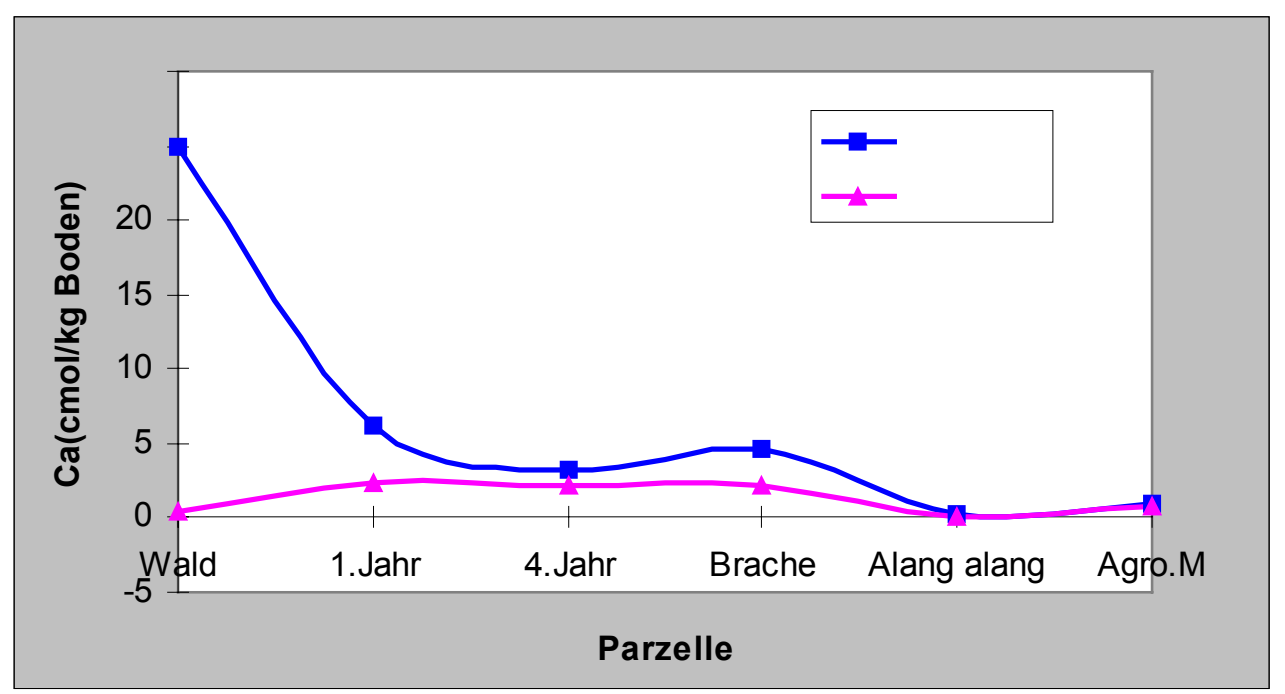

Abb. 41: Austauschbares Ca in Ober- und Unterboden 
Die KAKeff ist überwiegend von den Kationen Kalzium und Magnesium geprägt. Daher ergibt sich bezüglich der einzelnen Elemente ein vergleichbares Bild $\mathrm{zu}$ dem der gesamten KAKeff (Abb. 41 \& 42).

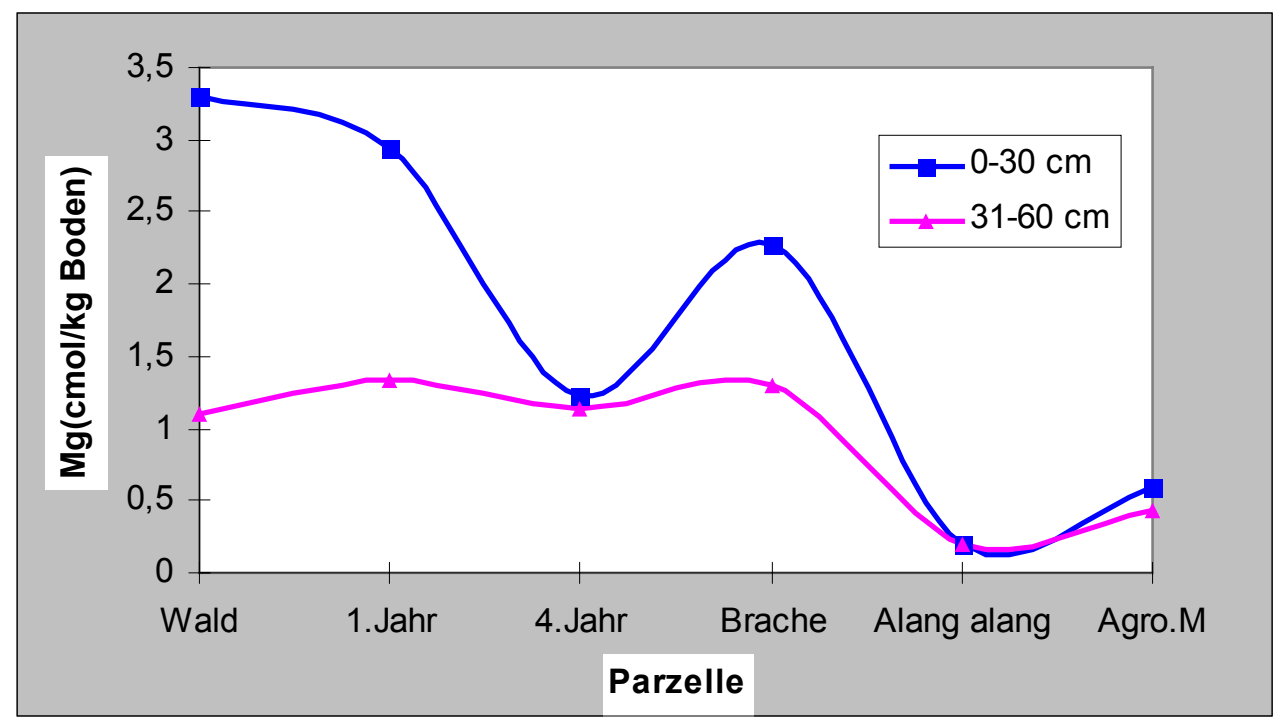

Abb. 42: Austauschbares Mg in Ober - und Unterboden

Zwischen dem $\mathrm{Ca}$ und der gesamten Austauschkapazität besteht eine sehr enge Korrelation (s. Abb. 43), die auch ersichtlich wird, wenn die Kurvenverläufe der zeitlichen Entwicklung der KAKeff und des austauschbaren Ca betrachtet werden. Das Bestimmtheitsmaß der Korrelation liegt mit $r^{2}=0,96$ sehr hoch.

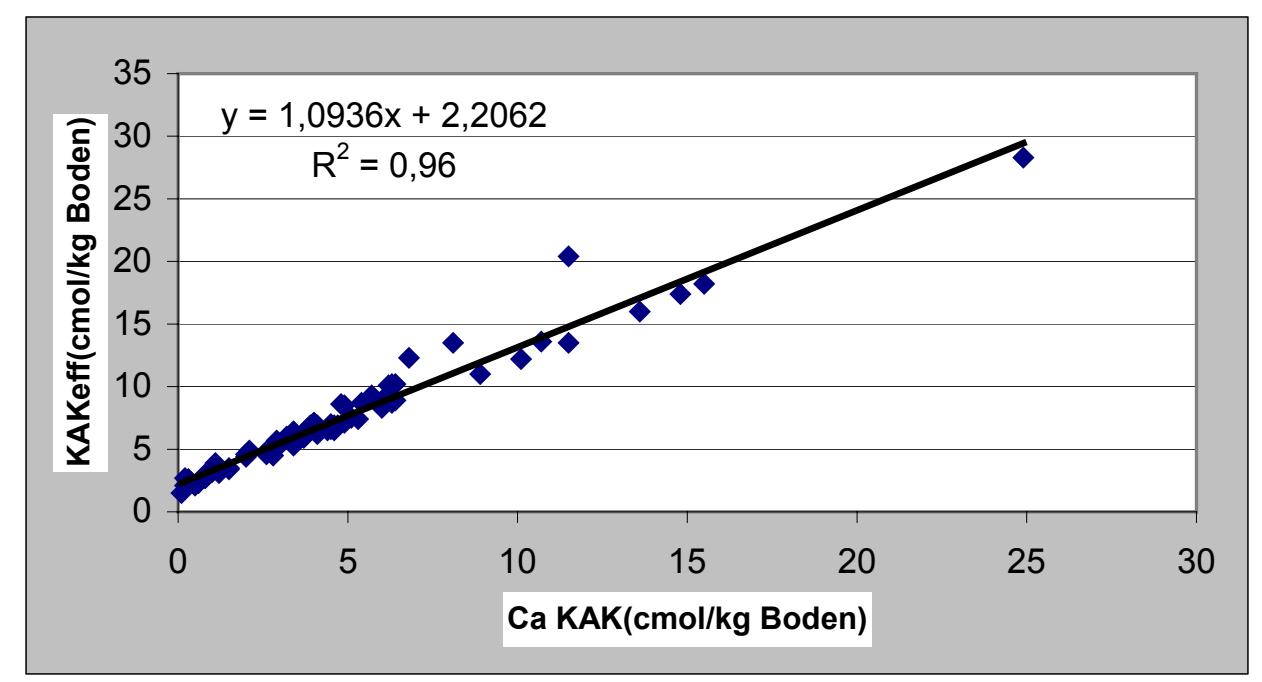

Abb. 43: Korrelation zwischen austauschbarem Ca und der Austauschkapazität

Wie SAIKH ET AL. (1998) auf Alfisolen in Indien feststellten, konnten auch in dieser Untersuchung $\mathrm{Ca}, \mathrm{Mg}, \mathrm{K}$ als Hauptbasen an der KAKeff ermittelt werden. Ca stellt 
mengenmäßig das Hauptaustauscherelement unter den austauschbaren Basen, gefolgt von Mg und K dar. Auch die Basensättigung der Böden entspricht im weitesten Umfang den hier dargestellten Ergebnissen und liegt mit vorwiegend über 80\% in den Oberböden sehr hoch. In Anlehnung an die weiter oben dargelegte Entwicklung der organischen Substanz werden auch die Ergebnisse des Austauschkomplexes interpretiert. Kultivierung verursacht eine hohe signifikante Verringerung der KAKeff des Bodens. Die Anteile der Basen $\mathrm{Ca}, \mathrm{Mg}$ und $\mathrm{K}$ an der gesamten Austauschkapazität ist ziemlich hoch im Wald und im 1. Jahr bzw. 4. Jahr nach Brandrodung ( $80-90 \%$, s. Anhang KAK). Nach längerer Zeit Bewirtschaftung (wie Parzelle Agroforst mit Mulch und Alang Alang) weisen die Böden in Ober- und Unterboden eine niedrige Basensättigung (22,9\%- 56\%) auf. Die KAKeff beträgt sogar nur noch 1,5-3,0 cmolc/kg Boden. Mit der Tiefe nehmen die Hauptbasenbildnern tendenziell ab, während parallel dazu die Gehalte an austauschbarem Aluminium steigen (vgl.Tab. Anhang KAK).

Verlust an organischer Substanz und KAKeff bzw. Basensättigung sind eng miteinander korreliert. Dies entspricht den Ergebnissen von TRAN DUC VIEN (1998) aus einem Feldversuch in der Provinz Hoabinh: Nach dem 2. Zyklus „slash -and Burn“ ist der pHWert von 5,2 auf 4 gesunken und der V-Wert für alle Landnutzungsarten war $<50 \%$ außer im 1. Jahr nach Brandrodung. Bei KARSTEN (2000) ist die Abnahme der KAKeff nach Entwaldung und einsetzender Kultivierung von bis zu 43\% sehr deutlich.

MÜLLER-SÄMANN (1986) beschreibt ebenfalls, dass die negativen Auswirkungen des Humusschwundes wichtige erwähnte fruchtbarkeitsbestimmende Eigenschaften des Bodens verändern. So ging zum Beispiel parallel zur Abnahme der organischen Substanz um 70\% die Kationenauschtauschkapazität (KAK) um 68\% zurück.

Abb. 44 zeigt einen sehr deutlichen Unterschied der KAKeff im Oberboden vor dem Anbau und nach der Ernte. Besonders deutlich ist es im 1. Jahr nach Brandrodung, wo die KAKeff von 10,7 cmol/ $\mathrm{kg}$ Boden vor dem Anbau auf nur noch 5,8 cmol/kg Boden nach der Ernte sinkt. Dagegen steigt die KAKeff auf der Parzelle Agroforst mit Mulch von 2,93 auf 11,43 cmol/kg Boden. KAKeff und damit auch Basensättigung werden in diesem Fall aufgrund besserer Humusbildung durch u.a. auf dem Boden verbleibendem Pflanzenschnitt, biologische Stickstofffixierung der Pflanzen und Schutz des Oberbodens durch Mulchen erhöht. 


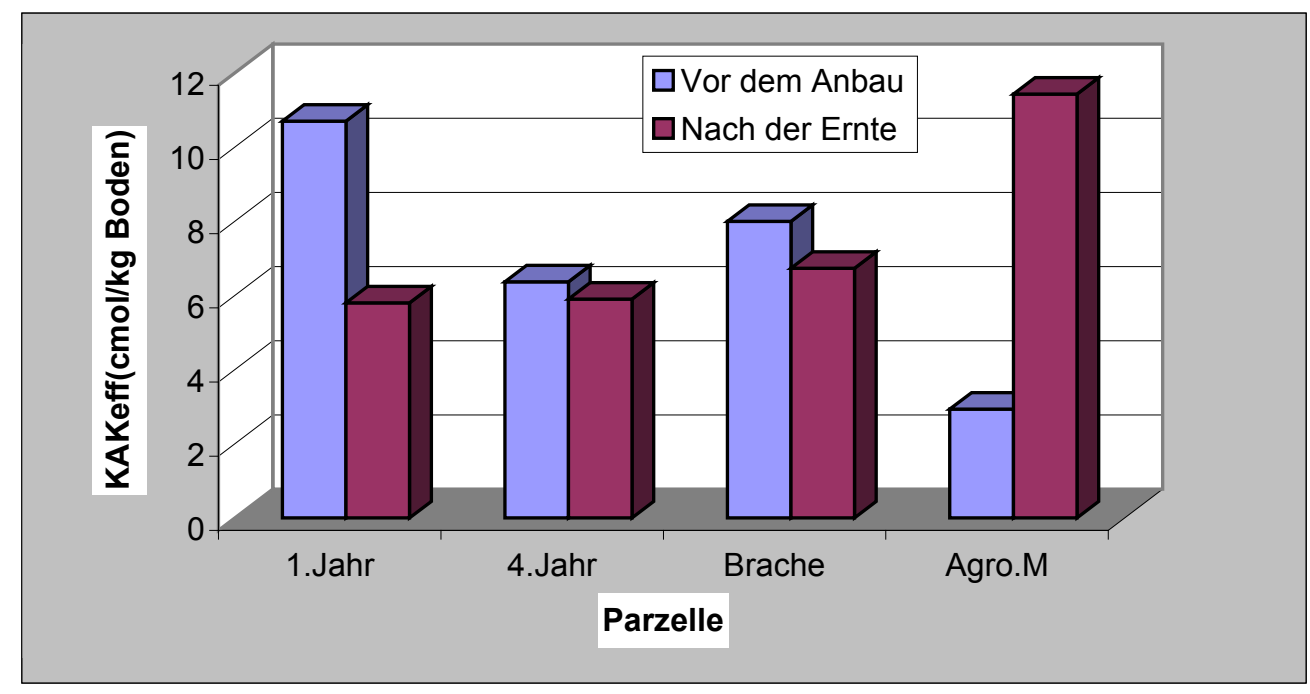

Abb.44: KAKeff vor dem Anbau und nach der Ernte im Oberboden

Die Kationenaustauschkapazität ist ein Maß für die Fähigkeit der Böden, pflanzenverfügbare Nährstoffe (Nährstoffkationen wie $\mathrm{Ca}^{++}, \mathrm{Mg}^{++}, \mathrm{K}^{+}, \mathrm{NH}_{4}{ }^{+}$) $\mathrm{zu}$ speichern und festzuhalten. Eine niedere Kationenaustauschkapazität $(<5-10$ m.e./ 100g Boden) bedeutet, dass die Böden bzw. die Tonminerale der Böden wenig negative Oberflächenladung besitzen, um an ihren Oberflächen Nährstoffe festzuhalten. Wenn auf solchen ferallitischen Böden mit geringer Nährstoffspeicher- und Austauschkapazität gedüngt wird, ist es zur Vermeidung von Nährstoffauswaschungen und zur Verhinderung von Nährstoffungleichgewichten wichtig, nicht viel Dünger auf einmal zu geben, sondern in mehreren kleinen Gaben, entsprechend dem zeitlichen Bedarf der Kulturpflanzen, zu düngen. (HARTGE \& HORN, 1991)

Zunehmend feuchttropische Verhältnisse bewirken zum einen, dass Tonminerale wie Kaolinit, Gibbsit und Halloysit gebildet werden, die eine geringe Kationenaustauschkapazität besitzen, zum anderen einen zunehmenden Abbau des mineralischen Bodenskeletts (des Ausgangmaterials), wodurch die Böden nur noch sehr wenig Mineralstoffreserven besitzen, die bei ihrer Verwitterung mineralische Nährstoffe freisetzen. Nach MoLL (1982) haben ferrallitische Böden nur noch weniger als 10\% verwitterbare Mineralreserve, meist sogar weniger als 5\%.

Böden der humiden Tropen mit Kaolinit und freien Oxiden in der Tonfraktion (geringer Anteil permanenter Ladung) haben deshalb einen geringen $\mathrm{pH}$ - Wert und V-Wert (NYE \& GREENLAND, 1960a).

Die potentielle Kationenaustauschkapazität ist aufgrund des hohen Tonanteils der Feinsubstanz selbst bei geringen Gehalten an organischer Substanz mittel bis hoch. Bei 
Gehalten der organischen Substanz von $>3 \%$ werden sehr hohe Austauschkapazitäten erreicht (bis 50,9me/100g). Drückt man die Austauschkapazität in Prozent des Tongehaltes aus, ergeben sich annäherungsweise 50-80me/100g Ton. Dies deutet auf die Anwesenheit von 2: 1 Tonschichtmineralen hin. Neben Illit (40me/100g) müssen daher größere Reserven Montmorrilonit (80-100me/100g) oder Vermiculit (100me/100g) in der Tonfraktion vorhanden sein (vgl. SCHERFFER UND SCHACHTSCHABEL, 1982).

Alle aufgeführten Aspekte werden stark vom klimatischen Parameter des Niederschlags geprägt. Die Wassererosion spielt dabei eine wichtige Rolle (s.Kap. 6.3). Durch Erosionsvorgänge auf der Hangfläche werden die Böden in ihrem Profilaufbau und in ihrem Nährstoffhaushalt verändert. Am Oberhang wird das Bodenprofil verkürzt und der Gehalt an organischer Substanz und Nährstoffen verringert. Am Unterhang wird das von der Hangfläche abgetragene Material mit organischer Substanz und Nährstoffen akkumuliert und, da es sich in den meisten Fällen um Feinmaterial handelt, sehr dicht gelagert (TEHRAMI, 1977).

Wie die Untersuchung von OvUKA (2000) im Bergland Kenyas herausgefunden hat, besteht ein hoher signifikanter Unterschied des Nährstoffgehaltes auf den verschiedenen Hangpositionen. Die Menge an K, P, Gesamtstickstoff und organischer Substanz sowie KAK, pH-Wert hängen von intensiver Landnutzung, Hangposition, Niederschlag und Bodenerosion ab. Maßnahmen des Erosionschutzes sind deshalb wegen der Hangneigung und der oftmals erosiven Niederschläge in den Tropen besonders zu beachten.

Auf den alten, kaolinit- und oxidreichen Böden der feuchtheißen Tropen stellt die niedere Kationenaustauschkapazität, neben dem Mangel an verwitterbaren Mineralen, ein großes Problem für die Bodennutzung dar.

KAKeff liegt auf Acrisolen meist unter 10 m.e./100 g Boden (teilweise sogar unter 5 m.e/100 g), wodurch die Böden nur wenig Nährstoffe festhalten und speichern können. Das ist besonders dann der Fall, wenn z.B nach Rodung und wenigen Jahren Ackerbau mit annuellen Kulturen auch noch die organische Substanz zurückgegangen ist, die gerade auf tropisch-verwitterten Böden erheblich zur Austauschkapazität beiträgt. Ein weiteres, wichtiges Merkmal fersiallitischer, kaolinitischer- oder humusreicher Böden ist die Tatsache, daß ihre Sorptionskraft (ihre Fähigkeit zur Ionenbildung) stark pHabhängig ist, dass heißt, sie besitzen einen hohen Anteil an variabler Ladung (KREBS, 1978; SCHEFFER\& SCHACHTSCHABEL, 1982).

YOUNG (1976) schreibt: „, Die Bedeutung der organischen Masse in tropischen Böden ist größer als die irgend einer anderen Eigenschaft des Bodens, wenn man einmal von der 
Feuchte absieht." Und SANCHEZ (1976) betont, dass die Erhaltung der organischen Masse „,von grundlegender Bedeutung für die Produktivität tropischer Böden“ ist.

Somit kann nach GEROLD (1991) für den Regenfeldbau in den wechselfeuchten Tropen aus der Bedeutung des Humushorizontes für die bodenchemische Fruchtbarkeit, den Bodenwasserhaushalt sowie für die Bodenerosionsresistenz die vorrangige Notwendigkeit der Anwendung humusschonender Anbausysteme gefordert werden. Die aufgezeigten Gradienten der organischen Substanz, des Kationenaustauschkomplexes sowie die Korrelation zwischen den austauschstarken Humuskomplexen und der Kationenaustauschkapazität machen im Hinblick auf eine nachhaltige Nutzung einen humusschonenden Anbau dringend erforderlich.

\subsubsection{Andere Parameter}

Die Gesamtgehalte der in Abb. 45 dargestellten Nährstoffelemente weisen im Zyklus der traditionellen Landnutzung deutliche Unterschiede auf. Es lässt sich wie folgt ihr Verhalten zusammenfassen:

- Die Gehalte aller Parameter im 1.Jahr nach Brandrodung sind sehr viel höher als auf allen andern Flächen. Aschedüngung führt hier zu diesen erhöhten Konzentrationen.

- Bei $\mathrm{K}, \mathrm{Mg}, \mathrm{Na}, \mathrm{Cr}, \mathrm{Ti}, \mathrm{Mo}, \mathrm{Cu}, \mathrm{Co}, \mathrm{Pb}$, ist folgende Verlaufzeit der Nährstoffabnahme zu erkennen: 1.Jahr $>$ Wald $>$ Brache $>4$.Jahr $>$ Agroforst mit Mulch $>$ Alang Alang.

- Bei Ca, Mn, Ni, Zn, S ist folgende Verlaufzeit der Nährstoffzunahme zu erkennen: Wald $>$ 1.Jahr $>$ 4.Jahr $>$ Brache $>$ Agroforst mit Mulch $>$ Alang Alang.

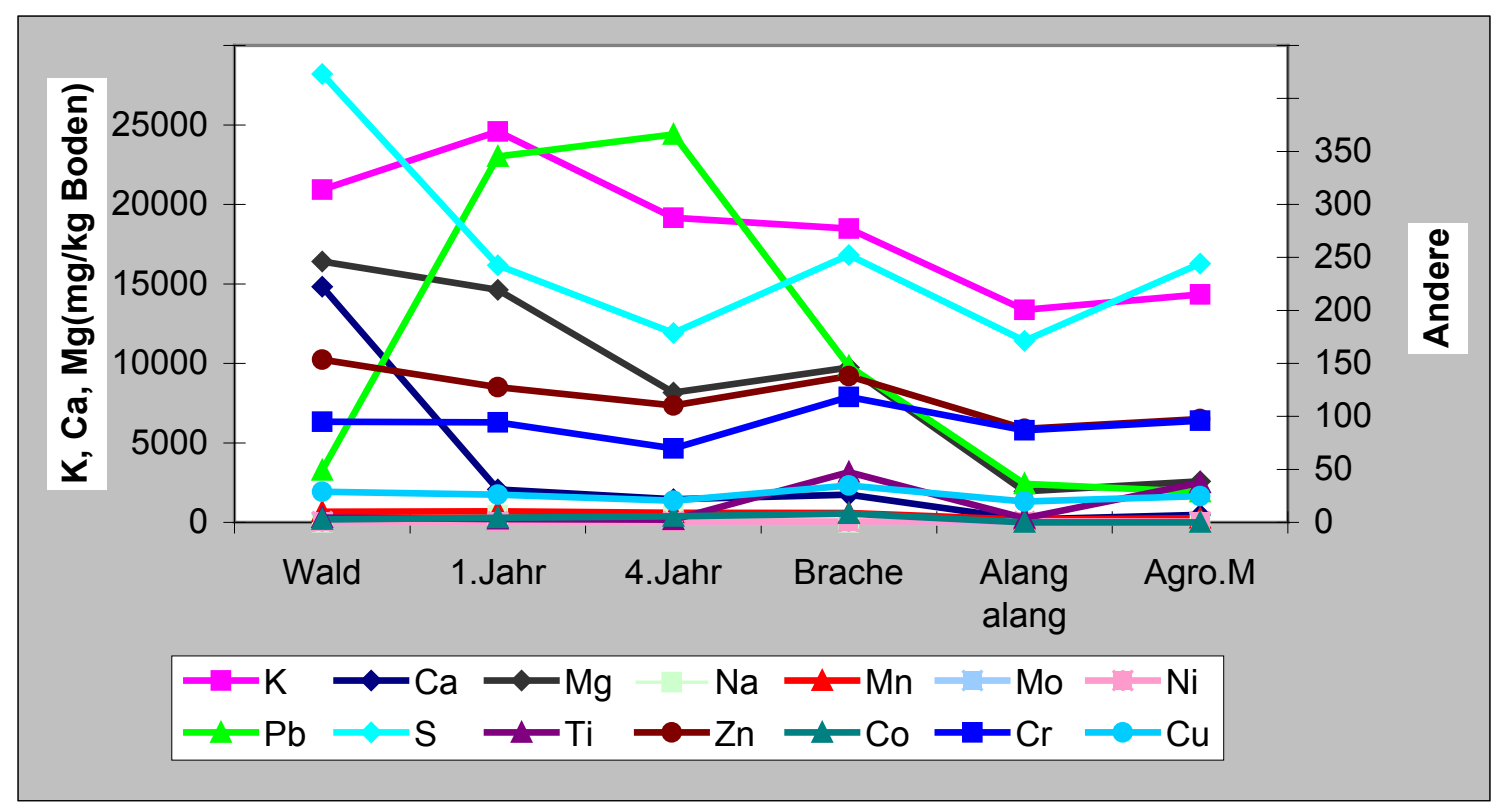

Abb.45: Gesamtgehalte in Abhängigkeit von Anbaudauer und Nutzungstyp 
Den Mangangehalt von Bodenlösung und Sorptionskomplex bestimmen die Art des Substrates (Tongehalt und organische Substanz), die Bodenfeuchte, der $\mathrm{pH}-$ Wert und die mikrobielle Aktivität. Je saurer und humusreicher ein Boden ist, desto größer ist der als Austauschmangan vorliegende Anteil des Gesamt-Mangans (FIEDLER, 2001)

Bei $\mathrm{pH}>6,5$ sind im Oberboden die gelösten Zink-Verbindungen zu 60-80\% durch die organische Substanz komplexiert. Mit abnehmendem pH-Wert nimmt die Mobilität des $\mathrm{Zn}$ im Boden zu. Bei $\mathrm{pH}<5$ liegen 99\% der Zn-Spezies in Form organischer Ionen bzw. Verbindungen vor $\left(\mathrm{Zn}^{2+}, \mathrm{Zn}(\mathrm{OH})^{+}, \mathrm{ZnSO}_{4}, \mathrm{ZnH}_{2} \mathrm{PO}_{4}{ }^{+}\right)$.

Kupfer ist eines der im Boden am geringsten löslichen Metalle. Die Bindungen des $\mathrm{Cu}$ an organische und anorganische Bodenbestandteile sind sehr fest. An der organischen Substanz können 25-75\% der Cu-Ionen sorbiert sein (FIEDLER, 2001).

Wesentliche ungünstige Fruchtbarkeitsmerkmale saurer Böden sind:

- Das Vorhandensein größerer Mengen austauschbaren und löslichen Aluminiums und Mangans bei pH-Werten unter 5,0. Größere Mengen aktiven Aluminiums wirken toxisch auf nahezu alle Kulturpflanzen.

- Saure Böden sind in der Regel arm an verfügbarem $\mathrm{Ca}, \mathrm{Mg}$, da die Kationen in Böden des humiden Klimas den wesentlichen Teil basisch wirkender Kationen darstellen und deshalb am stärksten ausgewaschen werden.

- Niederige pH-Werte erhöhen die Anionensorptionskapazität, reduzieren aber die KAK und damit den „Schutz“ des Bodens vor weitere Kationenauswaschung.

- Die Verfügbarkeit bzw. Löslichkeit der meisten Mikronährstoffe, wie Mn, Fe, Zn, B z.T. auch Cu, ist im sauren Bereich erhöht (PAGEL, 1981).

BRECKLING \& BIRKENMEIER (2000) behauptet: Der Vorteil des Abbrennens besteht darin, dass die in der Asche enthaltenen Mineralien den Kulturpflanzen direkt zur Verfügung stehen. Insbesondere Kalium, das in der Asche als Oxid oder Karbonat vorliegt, wirkt als mineralischer Nährstoff und hebt außerdem den pH-Wert des Boden an. Bei Werten des Ausgangbodens um pH 4-5 führt dies zu einer zusätzlichen Mobilisierung von Phosphat aus fixierten, sonst nicht pflanzenverfügbaren Bindungsformen. Ein Vorteil wird häufig auch darin gesehen, dass während des Brennens ein großer Teil der potentiell an Kulturpflanzen schädlichen Organismen abstirbt (z.B. praktisch alle epigäischen Insekten). Aufgrund der hohen Wiederbesiedlungskapazität ist dieser Effekt jedoch nur von kurzer Dauer. Die Nachteile und Gefahren des Abbrennens bestehen insbesondere in einem erheblichen Nährstoffverlust. Der größere Teil des organisch gebundenen Stickstoff und Schwefels verflüchtig sich während des Brennens in die Atmosphäre. Die 
löslichen Nährstoffe, insbesondere das mobilisierte Phosphat, unterliegen der Auswaschung, so dass nach dem Nutzungszyklus ein stark verarmter Standort zurückbleibt, der nur schwer regenerierbar ist, sofern es sich um eine großflächigeres Areal handelt.

MCGRATH (1987) stellt für die Brandrodung eine relativ schlechte Nährstoffbilanz auf: „Wenn dieser Biomassebeitrag mit berücksichtigt wird, erweist sich shifting cultivation als ein extrem unproduktives landwirtschaftliches System. Im Gegensatz zur vorherrschenden Auffassung ist im Brandrodungsverfahren das Problem unfruchtbarer Böden und starken Schädlingsdrucks nicht durch ein niedrig-input System sondern durch eines mit hohen Inputs gelöst. Brandrodung beinhaltet insofern eine grundlegend andere Strategie als Agroforst- oder Forstwirtschaft.

Weiterhin, wenn shifting cultivation heute die vorherrschende Anbauform ist, besagt dies nicht, dass das auch in der Vergangenheit überall galt. Die erfolgreichsten traditionellen Anbausysteme im Hinblick auf die Tragfähigkeit des Systems waren arbeits- und landintensiv, und sie waren mit einem aquatischen System verbunden. ... solche Systeme können hochproduktiv sein im Hinblick auf die Produktion pro Flächeneinheit. Außerdem scheinen sie unempfindlicher gegen Schädlinge und Krankheiten zu sein als terrestrische Systeme“.

\subsection{Schussfolgerung: Einflüsse des Brandrodungsfeldbaus auf die Bodenfruchtbarkeit}

Die vorliegende Untersuchung sollte mit ihren vorwiegend bodenchemischen Ergebnissen das Bild der Bodenfruchtbarkeit im Bergland Vietnams darstellen. Mit dem Eingriff des Menschen in das natürliche Waldökosystem am Standort durch Rodung, Brandrodung und Feldbau kommt es zu:

- einer Veränderung des pH-Werts und der Basensättigung. Es wurde eine sehr deutliche Abnahme nach der langjährigen landwirtschaftlichen Nutzung der Böden im Untersuchungsgebiet festgestellt ( $\mathrm{pH}-$ Wert von 6,48 auf 4,38; V-Wert von 99,6\% auf 28,9\%). Es ist ein Anzeichen für Bodendegradation.

- Das C/N Verhältnis wird enger 10-15. Die Humusqualität der untersuchten Parzellen ist als hoch einzustufen. Der Gesamtkohlenstoff und -stickstoff nimmt sehr deutlich ab nach der Brandrodung: organische Substanz von 8,1\% im Wald auf 4,4\% im 1.Jahr und auf 2,4\% nach dem 4.Jahr nach Brandrodung; $N_{t}$ von $0,38 \%$ im Wald auf $0,12 \%$ nach 
dem 4.Jahr nach Brandrodung. Die Ursache für die deutliche Abnahme der organischen Substanz wird durch die Bodenerosion und in der Volatisation der Elemente $\mathrm{C}$ und $\mathrm{N}$ gesehen.

- Die P- Verfügbarkeit nach Bray in Ober- und Unterboden ist als sehr gering einzustufen und die Abnahme der P- Verfügbarkeit ist sehr stark (P-Bray von 2,61 mg/kg Boden auf 0,42 $\mathrm{mg} / \mathrm{kg}$ Boden). Der Grund dafür liegt zum einem im Verlust von pflanzenverfügbarem Phosphors durch Bodenerosion und zum anderen in Eisen- und Aluminiumanreicherung in niedrigen $\mathrm{pH}-$ Wert-Bereichen, die eine verringerte Phosphorverfügbarkeit durch die irreversible Phosphatfixierung bewirken.

- Die KAKeff nimmt im Oberboden folgendermaßen ab: von $28 \mathrm{cmol} / \mathrm{kg}$ Boden unter Wald auf 10,7 cmol/ $\mathrm{kg}$ Boden nach dem 1. Jahr nach Brandrodung und auf $6,3 \mathrm{cmol} / \mathrm{kg}$ Boden nach dem 4. Jahr nach Brandrodung. Bei Alang Alang Bewuchs liegt die KAKeff sogar nur noch bei 2,1 cmol/kg Boden. Dabei folgt die KAKeff der Abnahme der austauschbaren Hauptbasen Ca und Mg. Die KAKeff ist als niedrig einzustufen. Mit der verringerten KAKeff sowie den abnehmenden Werten der organischen Substanz ist eine signifikante Herabsetzung des gesamten Austauschkomplexes - mineralogische Bodenmatrix und Humuskomplex-gegeben.

- Nach dem 3.Jahr der Anlage von Heckenreihen aus Tephrocia candida und Leuceana glauca und der Mischung annuelle Kulturpflanzen und Fruchtbäumen ist eine Tendenz der Rehabilitation der Bodenfruchtbarkeit erkennbar.

Die Bodenfruchtbarkeit im tropischen Regenwaldgürtel ist an sich von Natur aus beachtlich, wird jedoch außerordentlich labil, sobald der Mensch mit Kulturmaßnahmen in die natürliche Pflanzengemeinschaft eingreift. Wie Kap. 6.5 zeigt, werden rasch die Erträge bei mehrjähriger Ackernutzung absinken. Ursache des raschen Ertragsabfallen bei einjährigen Feldfrüchten im tropischen Regenwaldgürtel ist die durch heftige und hohe Niederschläge bedingte Wassererosion, die zu Bodenverschlämmung und -verkrustung, schlechter Bodendurchlüftung und Nährstoffauswaschung führt. Das andauernd feuchtheiße Klima hat auch einen raschen Humusabbau zur Folge und ebenso eine beträchtliche Verunkrautung sowie eine starke Verbreitung von Pflanzenkrankheiten und tierischen Schädlingen. Es sind also zahlreiche Probleme der Bodenfruchtbarkeit, die dem bodenbebauenden Menschen in der Regenwaldzone besondere Maßnahmen abverlangen. Durch die Rodung tropischer Wälder verändern sich Bodenklima, Wasser- und Nährstoffhaushalt erheblich. Bodendegradation ist oft, aber nicht zwangsläufig eine Folge (PRINZ, IN REHM 1986). Das Ausmaß der ökologischen Wirkungen wird durch eine Reihe 
weiterer Faktoren modifiziert (Rodungsart, Flächenausmaß, Hangneigung etc.), so dass im Einzelfall eine spezielle Bewertung angeraten ist.

Der Rückgang der Bodenfruchtbarkeit ist in zeitlicher Abhängigkeit von seinen Bodeneigenschaften (Humus-, Nährstoff- und Tongehalt, pH-Wert, Aggregatstabilität, Mikroorganismen), Fruchtfolge, Nutzungsintensität und bodenschützenden Maßnahmen zu sehen. Eine Bracheperiode zur Regeneration der Bodenfruchtbarkeit ist erforderlich. GEROLD (1991, a, b, c; 2002) stellt folgende aus anthropogen bedingten Nutzungseingriffen in den tropischen Wald resultierende pedoökologischen Folgen zusammen:

1. eine erhebliche Verminderung der vegetationsgebundenen Nährelementvorräte durch Nährstoffentzug der Kulturpflanzen und Brandwirkung

2. eine Öffnung des Bioelement-Kreislaufs mit Verlust des Wurzelfilzes und damit hohen Auswaschungsraten,

3. Rückgang der organischen Substanz und damit Verlust an Austauschkapazität sowie Verminderung der mineralisierten Nährelemente

4. Verlusten des gesamten Oberbodens durch Erosion

5. Bodenprofilverkürzung und Degradation des Humushorizontes durch Erosion EGGER \& KORUS (1995): Nährstoffversorgung und Mineralhaushalt stellt unter traditioneller Brandrodung in tropischen Bedingungen ein großes Problem dar. Zumal auf Granitverwitterung, die zu nährstoffarmen und stark sauren Böden führt, fehlt es stets an $\mathrm{N}$, oft an $\mathrm{P}$ und $\mathrm{K}$, stets sind $\mathrm{Ca}, \mathrm{Mg}$ sehr knapp, gelegentlich $\mathrm{S}$ und häufig gibt es Probleme mit Spurenelementen wie B, Zn, Mo. Die Böden haben wenig Innenbindefähigkeit. Sie muss, da nicht mineralisch gesichert, in Form gut zersetzter organischer Substanz als Humus geliefert werden- da dieser aber rasch weiter abbaut, muss entsprechend nachgeliefert werden.

Nach MÜLLER- SÄMAN (1986) stellt das regelmäßige Brennen mit der Überweidung zusammen die stärkste und häufigste menschliche Beeinflussung der Savannenökosysteme dar: Den kurzfristigen Vorteilen des Brennens (eine saubere Fläche, ein früherer Grasaustrieb, unter Umständen eine Einengung des $\mathrm{C} / \mathrm{N}$-verhältnisses) stehen langfristig schwerwiegende Nachteile entgegen.

Der bereits erwähnte Verlust an organischer Masse und die Reduktion des Bodenlebens führen zu Bodenverhärtungen, vermindertem Infiltrationsvermögen des Bodens, verminderter Wasserkapazität und als Folge davon auch oft zu großen Erosionsschäden, um nur einige der Negativeffekte zu nennen. 
Die Entnahme von nutzbaren Harthölzern führt zur Verarmung und Auflichtung der Vegetation. Brandrodung zerstört die Vegetation bis auf wenige Stockausschläge: Wird die Brandrodewirtschaft nach 1 bis 3 Jahren aufgegeben (meist nicht wegen rasch sinkender Bodenfruchtbarkeit, sondern primär wegen starker Verunkrautung und Vergrasung), so ist die Verbuschung und anschließende Regeneration des Waldes möglich LAUER (1956). Weitere Übernutzung durch Vieh und Brandrodewirtschaft führt zur Vernichtung der Bodenfruchtbarkeit, zu Bodenerosion, Devastierung und zum Verlust der Standortfruchtbarkeit.

Es ist daher umso dringlicher, dass die Anwendung angepasster Anbausysteme (z.B Agroforst mit Mulch) auf längerfristige Gleichgewichtszustände der Nährstoffversorgung im Bereich der organischen Substanz und des Austauschkapazität hinwirkt. Bodenbedeckung mittels organischer Substanz (Gründüngung, Mulchen, Verbleiben der Ernterste) wirkt als Input an Humus und Mineralien und wirkt höheren Auswaschungsraten entgegen.

Durch die Agroforstsysteme werden natürliche Landschaftselemente wie Gehölze, Hecken und Brachen verwendet. Die eingesetzten Pflanzenarten verstärken mit der Bestrebung nach Autochthonie die natürliche Wirkung. Allerdings sind immer noch viele der in den Agroforstsysteme eingesetzten Arten als nicht autochthon anzusehen, was der natürlichen Wirkung abträglich ist und durch weitere Anstrengungen dahingehend geändert werden sollte.

\subsection{Ergebnisse der Bodenerosionsmessung}

\subsubsection{Niederschlag}

\subsubsection{Erosivität des Niederschlages}

Die Erosivität des Niederschlages ist dessen potentielle Fähigkeit, einen Bodenabtrag hervorzurufen. Sie ist eine Funktion der physikalischen Eigenschaften des Niederschlages. Eine Rolle spielen hierbei die Niederschlagsmenge, die Niederschlagsdauer je Niederschlagsereignis und die Niederschlagsintensität.

Nordwest-Vietnam weist mit 1500 - $3000 \mathrm{~mm}$ eine sehr hohe jährliche Niederschlagsmenge auf. Der durchschnittliche Jahresniederschlag variiert innerhalb der Region und im zeitlichen Verlauf des Jahres. 80\% der Niederschlagsumme konzentrieren sich auf die Monate von April bis Oktober.

In Abb. 43 ist die monatliche Niederschlagsmenge während des Untersuchungszeitraumes von April bis Oktober 2001 nach eigenen Messungen dargestellt. Der Monat Juli weist 
dabei mit $384 \mathrm{~mm}$ die höchste Niederschlagsrate auf. Die Niederschlagshöhe zu Beginn (April) und Ende der Regenzeit (September) im Jahr 2001 (s. Abb. 46) entspricht mit 151,4 mm bzw. 157,8 mm in etwa dem Monatsmittel der Niederschläge der Jahre 19962000.

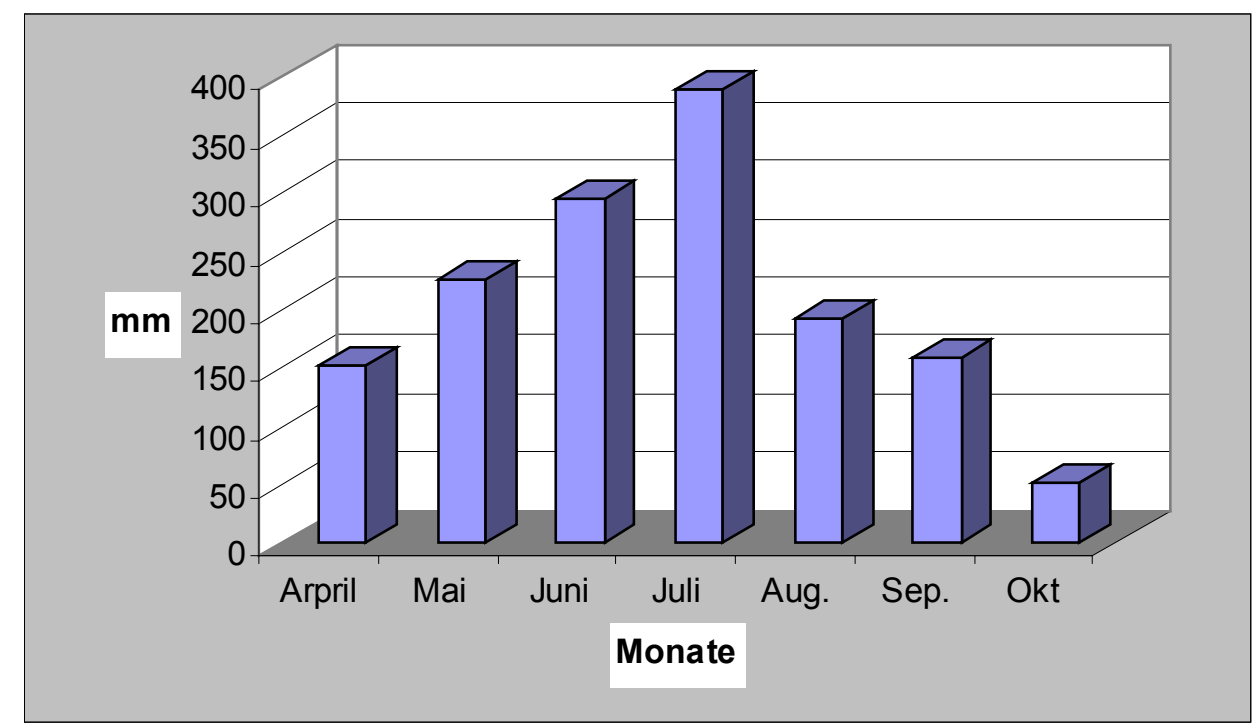

Abb. 46: Monatlicher Niederschlag von April - Oktober 2001 in Dia linh

Die Niederschlagssumme der Regenzeit des Jahres 2001 (April bis Oktober) entspricht mit 1462 mm dem Mittel der Regenzeiten der Jahre 1996-2000 (s. Kap. 3). In Abb. 47 ist erkennbar, dass die Monate Juni und Juli die höchste monatliche Niederschlagssumme aufweisen, was in der verhältnismäßig hohen Anzahl besonders niederschlagsreicher Ereignisse begründet ist. Während im Juni fünf Ereignisse mit jeweils mehr als $50 \mathrm{~mm}$ stattfanden, wurde im Juli nur drei Ereignisse dieser Art registriert. Die Verteilung der Niederschlagsereignisse über die Regenzeit war insgesamt gleichmäßig: durchschnittlich ein Niederschlagsereignis alle 4 Tage. Die Mittleren Tagesniederschläge liegen im Juni/Juli zwischen 50 und $70 \mathrm{~mm}$. Sie sind tendenziell im Juli höher als in anderen Monaten während der Regenzeit. THAI PHIEN \& N.T. SIEM (1999) stellten für das Hochland von Nordvietnam fest, dass die mittlere Niederschlagsmenge pro Regentag im Juli höher ist, als in allen anderen Monaten. 


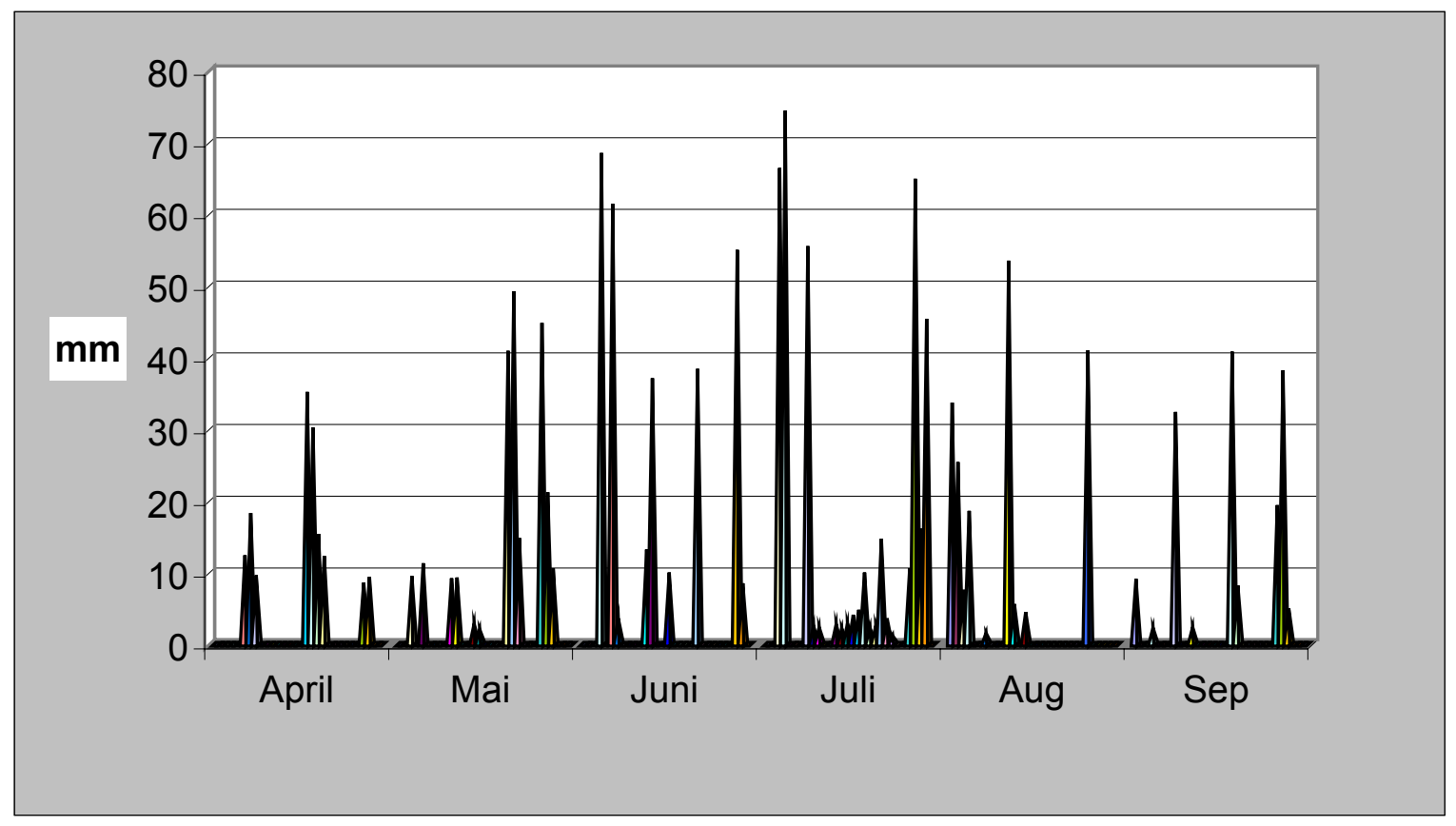

\section{Abb. 47: Die Verteilung und Höhe der Niederschlagsereignisse während der Regenzeit}

Bezüglich der Erosivität der Niederschläge in Vietnam gibt es bisher keine Untersuchungen. Es liegen aber Arbeiten aus anderen Regionen mit ähnlichen klimatischen Verhältnissen vor. MOHR \&VAN BAREN (1954) haben in ihrer Untersuchung in tropischen Klimaten wie Indonesien und den Philippinen festgestellt, dass $22 \%$ der Niederschläge als Starkregen fallen.

Obwohl Niederschlagsintensitäten von $150-200 \mathrm{~mm} \mathrm{~h}^{-1}$ bei relativ kurzer Niederschlagsdauer von z.B. 5-10 Minuten durchaus immer wieder vorkommen, sind die Niederschlagsintensitäten normaler Starkregen mit denen der gemäßigten Breiten durchaus vergleichbar. Da in den Tropen aber ein Großteil des Niederschlages in Form von Starkregen fällt und Niederschlagsintensitäten von 10 bzw. $25 \mathrm{~mm} \mathrm{~h}^{-1}$ oft überschritten werden, sind die tropischen Regen in ihrer Gesamtheit deutlich erosiver als die Regen in anderen Klimaten.

Nach Schwertmann et al (1990) wurde der Grenzwert für erosive Regen bei einer Niederschlagsintensität von $10 \mathrm{~mm} / \mathrm{h}$ festgelegt. Die Aufteilung des Niederschlags wurde durch das unten stehende Schema ausgedrückt, wobei Oberflächenabfluss stattfindet. Wenn es auf den Hang regnet, infiltriert ein Teil des Wassers in den Boden (I); ein Teil verdampft (E) und der restliche Teil fließt ab (Ao):

$$
\mathrm{Ao}=\mathrm{N}-(\mathrm{I}+\mathrm{E})
$$


Im Untersuchungsgebiet spielt der Verdampfungsfaktor (E) aufgrund der sehr hohen Luftfeuchtigkeit von $82 \%$ kaum eine Rolle. Während es regnet liegt er fast bei Null. Die Abflußmenge (Ao) wird deshalb umso größer und stärker sein, je höher die Niederschlagsmenge und je konzentrierter der Regen fällt.

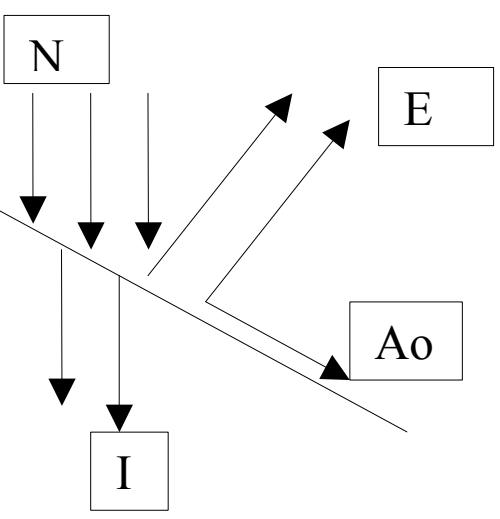

\subsubsection{Einfluss von Regenintensität und Niederschlagsmenge auf die Bodenerosion im Bergland.}

Die Höhe des Bodenabtrages steht allgemein in enger Beziehung zum Niederschlag und zwar teilweise durch die Ablösekraft der Regentropfen an der Bodenoberfläche und teilweise durch den Beitrag des Regens am Oberflächenabfluss. Bei der Erosion durch Oberflächenabfluss wird die Regenintensität als bedeutendste Einflussgröße angesehen.

Zur Charakterisierung eines Erosionsereignisses muss die Erosivität, d.h. die Erosionsfähigkeit der Niederschläge festgestellt werden. Da die Niederschlagsmenge wenig geeignet ist, die Erosionsfähigkeit eines Regens zu beschreiben (WISCHMEYER, 1959), wird die Intensität eines Regens zur Kennzeichnung der Erosivität herangezogen. Starkregen sind nach WeISCHET (1979) solche, die z.B. in 5 Minuten $5 \mathrm{~mm}$, in 60 Minuten $16 \mathrm{~mm}$ oder in 120 Minuten $24 \mathrm{~mm}$ Niederschlag bringen. Die maximale 30 Minuten-Intensität des Starkregens vom August 1997 betrug 28,7mm/h bei einer Gesamtniederschlagsmenge von 35,4 mm in $120 \mathrm{~min}$ in Thainguyen Provinz. Fast der gesamte Niederschlag von 25,8 $\mathrm{mm}$ fiel in $20 \mathrm{~min}$, davon $15 \mathrm{~mm}$ in $10 \mathrm{~min}$ (HA, SIEM \& PHIEN, 1998) 
In Tab.26 sind die beobachteten Niederschlagsereignisse auf den Testflächen in 2001 dargestellt. Bei einem Niederschlag von 20,2 mm mit einer Regenintensität von 0,07 mm/min wurde auf allen 4 Testflächen kein oberirdischer Abfluss und damit keine Bodenerosion festgestellt. Die gesamte Regenmenge von $202 \mathrm{~m}^{3}$ konnte in den Boden infiltrieren. Dagegen konnte bei einer Niederschlagsmenge von 68,5 $\mathrm{mm}$ und einer Regenintensität von $0,38 \mathrm{~mm} / \mathrm{min}$ ein starker Oberflächenabfluss mit starker Bodenerosion beobachtet werden.

Tab.26: Beziehung zwischen Niederschlag und Bodenerosion - Beobachtung einiger charakteristischer Niederschlagsereignisse

\begin{tabular}{|l|c|c|c|c|}
\hline $\begin{array}{r}\text { Bodenabtrag } \\
\text { kg/ha }\end{array}$ & 1. Jahr & 4. Jahr & Brache & $\begin{array}{l}\text { Agroforstsystem } \\
\text { mit Mulch } \\
\text { Miederschlag: } \\
\text { Menge(Intensität) }\end{array}$ \\
\hline $\begin{array}{l}38,4 \mathrm{~mm} \\
(0,116 \mathrm{~mm} / \mathrm{min})\end{array}$ & 160 & 260 & 0 & 0 \\
\hline $\begin{array}{l}68,5 \mathrm{~mm} \\
(0,38 \mathrm{~mm} / \mathrm{min})\end{array}$ & 6560 & 8240 & 220 & 360 \\
\hline $\begin{array}{l}20,2 \mathrm{~mm} \\
(0,07 \mathrm{~mm} / \mathrm{min})\end{array}$ & 0 & 0 & 0 & 0 \\
\hline $\begin{array}{l}25,4 \mathrm{~mm} \\
(0,19 \mathrm{~mm} / \mathrm{min})\end{array}$ & 580 & 920 & 20 & 60 \\
\hline
\end{tabular}

Weiterhin wurde beobachtet, dass eine enge Beziehungen zwischen Regenintensität, Regenmenge, Oberflächenabfluss und Bodenabtrag besteht.

\section{Tab:27: Beziehung zwischen Regenintensität und Oberflächenabfluss}

\begin{tabular}{|l|l|}
\hline $\begin{array}{l}\text { Regenintensität } \\
\mathbf{m m} / \mathbf{m i n}\end{array}$ & Oberflächenabfluss, Bodenabtrag \\
\hline$<0,07$ & $\begin{array}{l}\text { Kein oberirdischer Abfluss, keine Bodenerosion } \\
\text { Oberirdischer Abfluss entsteht nur bei Wassersättigung des } \\
\text { Bodens }\end{array}$ \\
$0,07-0,15-0,30$ & $\begin{array}{l}\text { Oberirdischer Abfluss findet statt, Bodenabtrag beginnt } \\
\text { Starker oberirdischer Abfluss mit starker Bodenerosion }\end{array}$ \\
\hline
\end{tabular}


In einigen Provinzen Nordvietnams weisen 41-62\% der Niederschläge eine für Bodenerosion relevante Stärke von $>25 \mathrm{~mm} / \mathrm{h}$ auf (HA, SIEM \& PHIEN, 1998).

Für das südliche Asien fand HUDSON (1971), dass Niederschläge erst bei einer Niederschlagsintensität von $>25 \mathrm{~mm} / \mathrm{h}^{-1}$ erosiv wirken.

Führen aber längeranhaltende geringe Niederschläge im Voraus zu Wassersättigung des Bodens, kann ein plötzlich auftretender Regen starker Intensität vollständig oberflächlich abfließen. In der Folge entstehen nicht nur starke Bodenerosion sondern auch Hangbewegungen z.B. in Form von Rutschungen und plötzliche Überschwemmungen: so z.B. bei dem Regen vom 13. Mai 2002 in Baccan, bei dem $192 \mathrm{~mm}$ fielen und durch die Überschwemmung 78 Menschen starben sowie zahlreiche Häuser zerstört wurden (www.VnExpress.vn).

Der Wunsch, die Bodenerosion in den landwirtschaftlich genutzten Gebieten im Bergland zu stoppen, lässt sich nicht verwirklichen, aber man kann durch Maßnahmen der Bodenbearbeitung, Kulturpflanzenpflege, Bodenbedeckung, Heckenpflanzungen etc. den Oberflächenabfluss und damit den Bodenabtrag vermindern. Nach BUI (1990), sind Böden aus Tonschiefer und Granit stärker anfällig für Bodenerosion als Böden auf Kalkstein. Ebenso sind die Erträge der Kulturpflanzen geringer und die Länge der Anbauperiode ist kürzer. Die Gründe dafür sind: Böden aus Kalkstein weisen im Oberboden eine gute Struktur und Textur mit hoher Wasserspeicherkapazität und Aggregierung auf. Der ca. $30 \mathrm{~cm}$ mächtige Oberboden (Ah) ist humos, feinkrümelig und locker. Da der Regen besser in der Boden infiltrieren kann, entsteht weniger Oberflächenabfluss. Darüber hinaus, bevorzugen die Bauern aufgrund langjähriger Erfahrungen und lokaler Kenntnisse westexponierte Hänge für den Anbau. An ostexponierten Hängen trocknet der Boden aufgrund höherer Sonneneintrahlung in den Nachmittagsstunden stärker aus und ist deshalb leichter erodierbar.

Es konnten verschiedene Formen der Bodenerosion im Bergland beobachtet werden: z.B flächenhafte Erosion, Rillenerosion, Gully-Erosion und sogar Massenbewegungen wie Hangrutschungen.

Neben der Gesamtniederschlagsmenge und der Niederschlagsintensität, übt auch der einzelne Regentropen durch seinen Aufprall auf den Boden eine erosive Wirkung aus. Diese wird durch physikalische Charakteristika der Regentropfen bestimmt d.h. durch die Tropfenmenge, -größe, die Größenverteilung sowie die Fallgeschwindigkeit. Obwohl bisher nur relativ wenige Untersuchungsergebnisse zur Charakterisierung der 
Regentropen tropischer Niederschläge vorliegen, kann man feststellen, dass der Tropfendurchmesser im Mittel mit etwa 2-4 mm relativ groß ist.

Im Falle von Ibadan (Nigeria) konnte LAL (1987) feststellen, dass die Häufigkeit von Niederschlägen mit Tropfengrößen über $3 \mathrm{~mm}$ in den Monaten April bis Juli und im Oktober hoch ist. Es handelt sich dabei um Monate mit 14 bis 24 Niederschlagsereignissen. In den anderen Monaten mit einer wesentlich geringeren Zahl an Niederschlagsereignissen blieb die Tropfengröße teilweise deutlich unter $3 \mathrm{~mm}$. Zu der relativ großen Tropfengröße kommt hinzu, dass Starkregen in den Tropen oft mit relativ hohen Windgeschwindigkeiten gekoppelt sind. Der Wind verändert durch eine horizontale Komponente die Tropfengeschwindigkeit und verstärkt sehr oft die Prall- und Planschwirkung.

Aus der oben dargelegten Niederschlagscharakteristik tropischer Regen resultiert eine relativ hohe kinetische Energie des Einzelregens wie auch des erosiven Niederschlages pro Monat bzw. pro Jahr. LAL (1987) z.B. berichtet von einer Energiesumme eines Einzelregens in Ibadan im Jahre 1979 in der Größenordnung von 7000-8000 J/m². Die Häufigkeit von Niederschlagsereignissen mit einer kinetischen Energie von 500 Joules und mehr pro Regen ist relativ groß, dies trifft auch für die monatliche kummulative kinetische Energie der Niederschläge am Standort Ibadan (Nigeria) zu, wie LAL (1987) dargestellt hat. Ackerbaulich von Bedeutung ist, dass sich auch die erosiven Niederschläge ganz offensichtlich auf einige Monate im Jahr konzentrieren und nicht gleichmäßig über das Jahr verteilt sind.

Zusammenfassend kann man festhalten, dass jahreszeitlich begrenzt, tropische Regen potentiell eine hohe Erosivität besitzen und damit viele Standorte mit hohen Hangneigungen in den Tropen eher ackerbaufeindlich als - freundlich sind. Allerdings wird, wie oben bereits aufgezeigt, der Bodenabtrag nicht nur durch die Erosivität des Niederschlages allein, sondern ganz besonders auch durch die Erodibilität des Boden beeinflusst.

$\mathrm{Zu}$ Beginn der Regenzeit ist die Intensität und die Menge des Regens im Vergleich zum Ende der Regenzeit geringer, trotzdem ist der Bodenabtrag doppelt so groß (s Tab.28). Der Grund dafür liegt darin, dass der Boden zu Beginn der Regenzeit einer langen Trockenperiode ausgesetzt war, und somit bei noch geringer Bodenbedeckung leicht erodiert werden kann. Ein weiterer Grund liegt darin, dass das Wurzelwerk der noch jungen Maispflanzen noch nicht weit genug ausgeprägt ist, um den Boden festzuhalten. 
Tab.28: Vergleich von Bodenabtrag zu Beginn und Ende der Regenzeit

\begin{tabular}{|c|c|c|c|c|c|c|}
\hline \multirow{2}{*}{$\begin{array}{l}\text { Zeitraum der } \\
\text { Beobachtung }\end{array}$} & \multirow{2}{*}{$\begin{array}{c}\text { Niederschlag } \\
\text { (mm) }\end{array}$} & $\begin{array}{l}\text { Regen- } \\
\text { intensität } \\
(\mathbf{m m} / \mathbf{m i n})\end{array}$ & $\mathbf{4 . ~ J a h r}$ & $\mathbf{1 . ~ J a h r}$ & Brache & $\begin{array}{l}\text { Agrofors } \\
\text { t } \\
\text { mit } \\
\text { Mulch }\end{array}$ \\
\hline 20.05.2001 & 30,6 & 0,21 & 16,1 & 12,8 & 0,5 & 0,75 \\
\hline 21.05 .2001 & 35,5 & 0,23 & 18,2 & 15,0 & 0,65 & 0,95 \\
\hline 18.09 .2001 & 40,8 & 0,32 & 9,7 & 7,1 & 0,15 & 0,40 \\
\hline 27.09 .2001 & 38,2 & 0,28 & 7,6 & 3,8 & 0,1 & 0,3 \\
\hline
\end{tabular}

Auf den zur landwirtschaftlichen Nutzung bestimmten Arealen werden die Primär- oder Sekundärwälder zunächst Mitte der Trockenzeit abgeholzt und am Ende derselben abgebrannt. Von diesem Zeitpunkt bis zur Entwicklung einer ausreichenden Vegetationsdecke (etwa zwischen Februar und Ende März) sind diese Flächen den erosiven Niederschlägen ungeschützt ausgesetzt. Gerade am Anfang der Regenzeit führen die Niederschläge zu hohen Bodenabträgen, welche dann aber mit zunehmendem Bedeckungsgrad des Pflanzenbewuchses zurückgehen (Abb.48 \& Tab. 29). In ähnlichem Zusammenhang berichten Cuevas \& Duma (1998) aus Los Banos Philippine, dass bedeckungsfreie Bergfelder (gerade bei und kurz nach der Aussaat) stark der splasherosion zum Oper fallen. Ferner berichten sie, dass Oberflächenabfluss, bedingt durch höhere Intensitäten der Niederschläge, in tropischen Regionen früher als in gemäßigten Regionen einsetzt. Zu Beginn der ersten Messperiode 1990/91 im Alto Beni-Gebiet bei unbedeckter Bodenoberfläche der Reisparzelle verursachten gerade die ersten Niederschläge einen stärkeren Bodenabtrag als zu Beginn der zweiten Messperiode 1991/92 bei einer Bodenbedeckung von ca. 40\%. Das Aufprallen von Regentropfen auf die ungeschützte Bodenoberfläche zerstörte 90/91 schnell die Bodenaggregate, löste die Bodenpartikel ab und spitzte sie in verschiedene Richtungen (splash erosion), so dass sich die Bodenoberfläche schneller versiegelte (SMITH ET AL, 1990, ROTH 1991, ROTH ET AL, 1986). Nach Roth, (1991) ist dieser Prozess bei getrockneten Böden auf die Luftsprengung der Aggreate zurückzuführen. Nach der Abholzung und dem Brennen des 
Primär- oder Sekundärwaldes sowie zunehmender Bodenbearbeitung geht aber die Infiltrationsrate des Bodens stark zurück (LAL 1976, ROTH ET AL 1986). Aus diesen Gründen steigt der Oberflächeabfluss und dementsprechend der Bodenabtrag.

Erosionsstudien in Mittel-Bedfordshire in England (MORGAN et al., 1986) belegen, dass in der Zeit von 1973 bis 1979 80\% der Erosion während 13 Starkregen auftraten. Der größte Bodenverlust mit $21 \%$ des Gesamtabtrags entstand durch einen Starkregen mit $57,2 \mathrm{~mm}$ Niederschag. Derartige Starkregen treten in England mit einer Häufigkeit von 24 pro Jahr auf.

Erosionsmessungen in der Nähe von Ibadan, Nigeria zeigten, dass die Hälfte des jährlichen Bodenverlustes mit 2-7 Unwettern verbunden ist, was die Häufigkeit von Starkregenereignissen in Beziehung zur Stärke der Hangerosion setzt. HusON (1981) betont ebenfalls die Rolle der Stärke eines Unwetters für den Bodenabtrag. Gestützt auf eigene Forschungen in Zimbabwe stellt er fest, dass 50\% des jährlichen Bodenverlustes durch nur zwei Starkregen verursacht wurden und dass in einem Jahr 75\% des Abtrags in 10 Minuten stattfand.

THAI PHIEN (1998) hat bei Untersuchungen in Vietnam herausgefunden, dass Niederschläge mit einer Intensität von $>25 \mathrm{~mm} / \mathrm{h}$ zu 55,6\% am Bodenabtrag beteiligt waren. Als besonders schwerwiegend erwiesen sich Niederschläge $>75 \mathrm{~mm} / \mathrm{h}$, die ca. 8,5\% des Jahresniederschlags entsprechen. Derartige Starkregen führen zu Gully-Erosion und Massenbewegungen wie Muren und Hangrutschungen.

Die Aufgabe besteht deshalb darin, für die Bewirtschaftung im Bergland angepasste Maßnahmen zur Verminderung von Bodenerosion zu finden.

\subsubsection{Bodenabtrag}

In Abb. 48 sind die gemessenen Abtragsraten der verschiedenen Testparzellen zusammenfassend dargestellt. Die Bodenabtragsraten variieren sehr stark in Abhängigkeit von der Bodennutzung und der Niederschlagshöhe.

Der Bodenabtrag im Maisanbau ist am höchsten: von $43 \mathrm{t} / \mathrm{ha} / \mathrm{a}$ auf der Parzelle 1.Jahr nach Brandrodung bis 58,3 t/ha/a im 4. Jahr nach Brandrodung. MoRGAN (1999) erklärt, dass die Nachwirkung des Waldes auf den Humusgehalt und die Aggregatstabilität des Bodens im allgemeinen für das erste Jahr des Anbaus vorhält. In Sabah, Malaysia (SINAJIN, 1987) betrug der Bodenverlust von Maisparzellen im ersten Jahr 0,05 t/ha in der Zeit vom Auspflanzen bis zur Ernte, stieg aber im dritten Anbaujahr auf 2,02 t/ha an. 
Der niedrigste Gesamtbodenabtrag wird auf der Testparzelle im Agroforstsystem mit Mulchbedeckung erreicht: 3,686 t/ha/a.

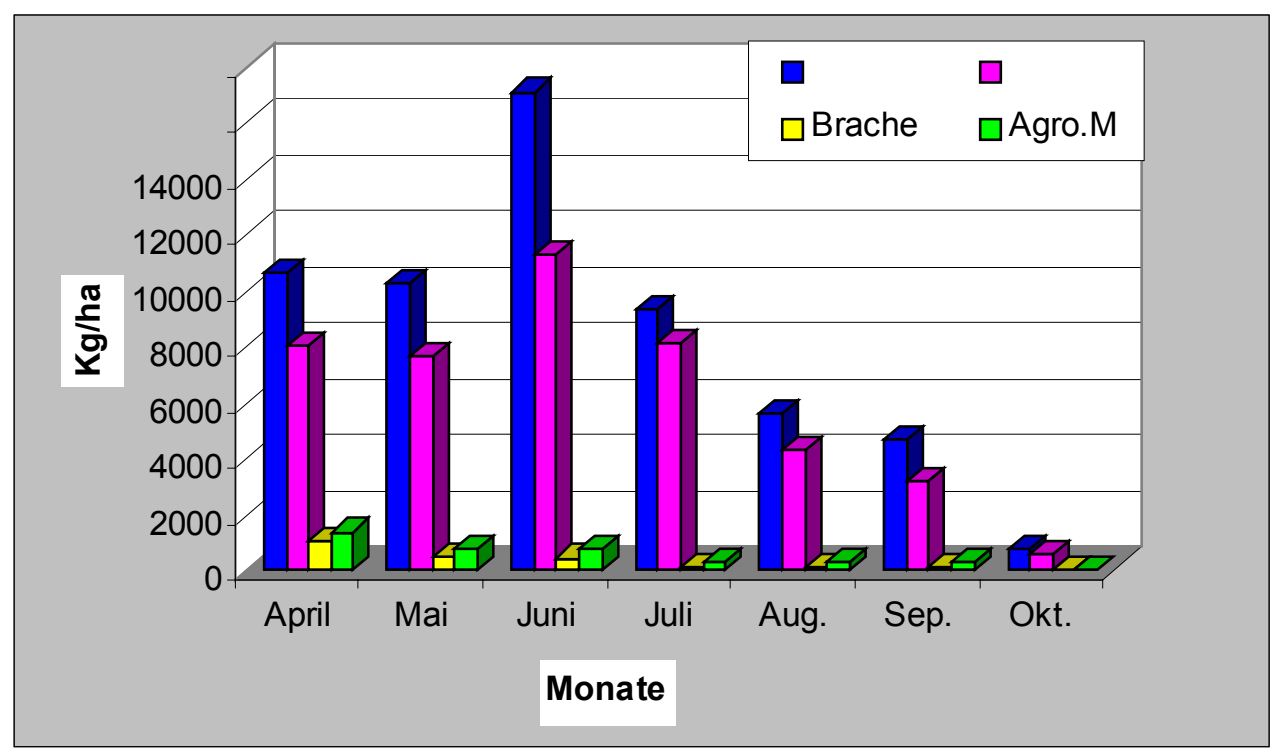

Abb. 48: Bodenabtrag verschiedener Landnutzungssysteme in 2001

Vergleicht man den Gesamtbodenabtrag der Parzellen unter Erosionschutzmaßnahmen und Maisanbau, so stellt man fest, dass Agroforstsysteme mit Mulch einen verringerten Bodenabtrag aufweisen und so wird deutlich, welchen erheblichen Beitrag zur Bodenerhaltung (Reduktion des Bodenabtrags auf weniger als 1/10) traditionelle Brandrodung ohne Erosionsschutzmaßnahme leisten können.

Aus Abb. 48 sieht man, dass der Bodenabtrag im Juni am höchsten ist, obwohl der Juli die höchste Niederschlagsmenge aufweist. Der Grund dafür liegt mit darin, dass im Juli die Bodenbedeckung am größten ist. Ein weiterer Grund dafür ist, dass im Juni mehr Ereignisse von jeweils mehr als $50 \mathrm{~mm}$ stattfanden.

Innerhalb der Parzellen mit Erosionsschutzmaßnahmen ist zu erwähnen, dass das Mulchen am Anfang des Versuchs noch einem besseren Erosionsschutz bietet als Hecken sowie Alley croping (Obstkulturpflanzen wie Longan, Litchi, Orange).

Das mit einer 0,5 $\mathrm{m}$ breiten Tephrocia candida- und Leuceana glauca- hecke angelegte Alley cropping erreicht nur 3,68 t/ha/a Bodenabtrag. Der Einfluss wird umso größer, je mehr die Bodenoberfläche bedeckt wird.

Die schützende Wirkung des Bedeckungsgrades auf der Brache zeigt sich deutlich durch eine Verringerung des Bodenabtrags. Die Rehabilitation der Bodennährstoffe verläuft in einem 4- jährigen Zyklus zwischen Maisanbau und Brache. 
SIEM \& PHIEN (1999) haben in Untersuchungen festgestellt, dass der Bodenabtrag von Niederschlagshäufigkeit, Niederschlagsintensität, Hangneigung, Bodenart, Bodenbedeckung und Landnutzung abhängt. Im Wald findet ein nur geringer Bodenabtrag von ca. 2-5 t/ha/a statt. Unter Teepflanzen beträgt der Abtrag 3-4 t/ha/a, bei Maniok oder Kulturpflanzen 40-100 t/ha/a je nach Bodenbedeckung. Bei fehlender Bodenbedeckung ist der Bodenabtrag je nach Bodenart mit 80-100 t/ha/a am größten. Von TRAN (1996) und BUI (1990) wurden sogar Bodenabtragsraten in slash- and- burn (Mais- und Reisanbau) von 75-100 t/ha/a gemessen.

Untersuchungen aus dem Hochland von Kenia und aus Tansania zeigen, dass bei einjährigen Feldfrüchten und besonders Mais, Bohnen und Sorghum hohe bis sehr hohe Erosionsraten erreicht werden (MESTMACHER,1989). Mit Mais bepflanzte Äcker liegen zudem zu Beginn der Regenzeit etwa einen Monat ungeschützt. Der Deckungsgrad steigt erst während des zweiten Monats (MORGAN,1999).

Die erosionshemmende Wirkung des Mulchens für die Verringerung von Erosion wurde durch Felduntersuchungen von BORST \& WOODDBURN (1942) aufgezeigt, die herausfanden, dass auf einem Schluff-Lehm-Boden auf einem $7^{\circ}$ - geneigten Hang der jährliche Bodenverlust für nicht bewirtschaftete, unbedeckte Flächen 24,6 t/ha betrug, aber nur 1,1 t/ha für Flächen, die mit Strohmulch in einer Menge von 5t/ha bedeckt waren. LAL (1976) stellt fest, dass die Bedeckung eines Alfisols auf einem $6^{\circ}$ - geneigten Hang mit 6 t/ha Strohmulch zu einem Jahresbodenverlust von 0,2 t/ha führte, verglichen mit 23,3 t/ha für unbedeckten Boden. Die Aufbringung von Mulch aus Alang Alang Gras (Imperata cylindrica) in einer Menge von 3t/ha bei Mais auf einem sandigen Lehmboden auf einem $4^{\circ}$ - geneigten Hang auf der Experimental Farm der Universität Pertanian Malaysia verringerte den Bodenverlust zwischen Oktober 1978 und Juli 1979 von 7,5 t/ha für Mais ohne Mulch auf 0,5 t/ha (MOKHTARUdDin \& MAENE, 1979).

Obwohl so niedrige Bodenverluste nicht immer erreicht werden, ist das Ausmaß, mit dem Mulch Erosion vermindert, allgemein beeindruckend. Ein Grasmulch von 4t/ha, aufgebracht auf einen Schluff-Lehm-Boden auf einem 4,5 - geneigten Hang in der Nähe von Dehra Dun, Indien, verringerte den Jahresbodenverlust unter Mais auf 5,0 t/ha, verglichen mit 22,4 t/ha für Mais unter herkömmlicher Bodenbearbeitung hangauf- und abwärts (KHYBRI, 1989). In der Nähe von Pakhribas im östlichen Nepal ergab ein von Grasmulch 15 t/ha auf einem sandigen Lehmboden mit $3^{\circ}$ - Hangneigung unter Mais einen Jahresbodenverlust von 16,9 t/ha, verglichen mit 32,8 t/ha bei der traditionellen Methode der Maisaussaat, dem ein zweimaliges Hacken folgte (SHERCHAN ET AL., 1990). 
Mulchen kann auch unter Baumkulturen betrieben werden. Durch die Bedeckung der Wirtschaftswege mit geschnittenen Palmwedeln in einer Ölpalmenplantage wurde in Johor, Malaysia, der Jahresbodenverlust auf 4,2 t/ha gegenüber 14,9 t/ha auf ungeschützten Wegen verringert (MAENE ET AL., 1979). Grasmulch, aufgebracht zwischen den Reihen junger Teesträucher auf einem $6^{\circ}$ - geneigten Hang auf einem Ultisol in der Nähe von Kericho, Kenia, verminderte den mittleren Jahresbodenverlust über einen Zeitraum von 3 Jahren auf 0,68 t/ha verglichen mit 210,8 t/ha bei unbedecktem, von Hand bearbeiteten Boden zwischen den Reihen (OTHIENO, 1978).

Mulchen bedeutet meist, dass Pflanzenreste auf der Bodenoberfläche verteilt werden, aber diese Methode bringt Probleme beim Drillen oder Pflanzen durch die Mulchschicht hindurch mit sich, es sei denn man verfügt über spezielle Geräte. Der Mulch kann auch das Auflaufen verzögern und, außer wenn die Mulchbedeckung $>90 \%$ beträgt, das Unkrautwachstum fördern. Eine Alternative besteht darin, den Mulch einzuarbeiten. Obwohl dies die Oberflächenbedeckung verringert, helfen die Mulchbestanteile, den Boden zu binden, indem sie die Wirkung von Pflanzenwurzeln simulieren, und die Infiltrationsraten zu erhöhen. DULEY \& RUSSEL (1943) fanden heraus, daß eingearbeitete Pflanzenreste den Jahresbodenverlust von 35,7 t/ha auf einem unbedeckten Boden auf 9,9 t/ha senkten.

Neben der Erosionsschutzwirkung werden als weitere Vorteile des Mulchens genannt:

- Zufuhr von Pflanzennährstoffen (MÜLER-SÄMANN, 1986; SIEM \& T.PHIEN, 1999)

- Mobilisierung von Nährstoffen durch Aktivierung des Bodenlebens (z.B: Rhizobien, Mycorrhiza) (DERPSCH AT AL, 1988; MÜLER-SÄMANN, 1986; SIEM \& T.PHIEN, 1999)

- Steigerung der Kationenaustauschkapazität aufgrund der Humusmehrung (DERPSCH AT AL, 1988; MÜLER-SÄMANN, 1986)

- Unterdrückung des Beikrauts (KUYPERS, 1987 ; MÜlER-SÄMANN, 1986; PRINZ, 1986)

- Reduzierung der Evaporation (BREBURDA, 1983; KUYPERS, 1987 ; MÜLER-SÄMANN, 1986; PRINZ, 1986)

Natürliche, stabile Bodenaggregate entstehen durch das Zusammenspiel von biologischchemischen und physikalischen Prozessen. Durch Pilzhyphen und Ausscheidungen der Bodenlebewesen werden Bodenteilchen miteinander verbunden, was die Erosionsgefahr verringert. Die absterbenden Wurzel einer Gründüngung versorgen Bodenlebewesen in Tiefen von mehr als $10 \mathrm{~cm}$ mit der notwendigen energiereichen Nahrung und sorgen für die bodenschützende , Lebendverbauung“( PREUSCHEN \& BERNATH, 1983). 
BUI (1990) hat zwischen 1963-1971 viele Versuche durchgeführt, um den Stand der Bodenerosion zu bewerten. Die traditionale Landnutzung der ethnischen Minderheit im Bergland, egal welche Bodenart und egal welche Kulturpflanzen betrugen $100-350$ t/ha/Jahr. In vielen Regionen haben die Bauern nur 1-2 Jahre Kulturpflanzen angebaut, danach müssen sie brach fallen lassen, und sich einen anderen Ort suchen, weil der Oberboden erodiert ist, und das Anstehende hervortritt.

Aufgrund der hohen Abtragsraten auf den kleinbäuerlich bewirtschafteten Parzellen in Butara (246 Tonnen pro Hektar und Jahr im fünfjährigen Mittel) kommt es ohne wirkungsvollen Erosionsschutz zu einer irreversiblen Degradation der Böden mit deutlichen Auswirkungen auf die Standortproduktivität, d.h. zu einer deutlichen Verarmung des obersten Profilabschnittes an Nährstoffen und an organischer Substanz. Allein durch den Übergang zu Baumintegration, Mischkultur, Gründüngung und Kompostierung (Pazelle1) läßt sich die fortschreitende Bodendegradation- bei einer Abtragsrate von 83t/ha/a - nicht wirkungsvoll aufhalten (MÜLLER- SÄMANN 1986). Die zusätzliche Integration höhenlinienparalleler Leuguminosenhecken erlaubt es dagegen, Oberflächenabfluss und Bodenabtrag auf ein unter den gegebenen Standortbedingungen tolerierbares $\mathrm{Ma} 3 \mathrm{zu}$ begrenzen. Vergleicht man den auf Parzelle 2 gemessenen Bodenabtrag (10,8 t/ha/a bei traditionellem Maniokanbau zwischen Tephrocia candida Hecken) mit dem auf der kleinbäuerlich bewirtschafteten Parzelle (Maniok keine Hecken, $246 \mathrm{t} / \mathrm{ha} / \mathrm{a})$, so wird deutlich, welchen erheblichen Beitrag zur Bodenerhaltung (Reduktion des Bodenabtrags auf weniger als fünf Prozent) allein Hecken auf Mikroterrassen leisten können.

Bodenerhaltungseffekte von Agroforstsystemen wurden in Zusammenhang mit Heckenreihen von Tephrocia candida in Nordthailand studiert, in dem Testparzellen (HURI \& NUNTAPONG, 1983) auf einem 54\% geneigtem Hang konstruiert wurden. Die Agroforst - Parzelle wurde mit einer Mischung verschiedener Baumsorten wie Kaffee, Obst (in den Streifen) und Getreide wie Trockenreis, Mais, Erdnuss bepflanzt. Die Bodenverluste auf der Agroforst - Testparzelle waren 10-20 t/ha/a und damit 4-9 mal niedriger, als entsprechender Bodenverlust bei traditionellem Trockenreis ( $89 \mathrm{t} / \mathrm{ha} / \mathrm{a})$. 


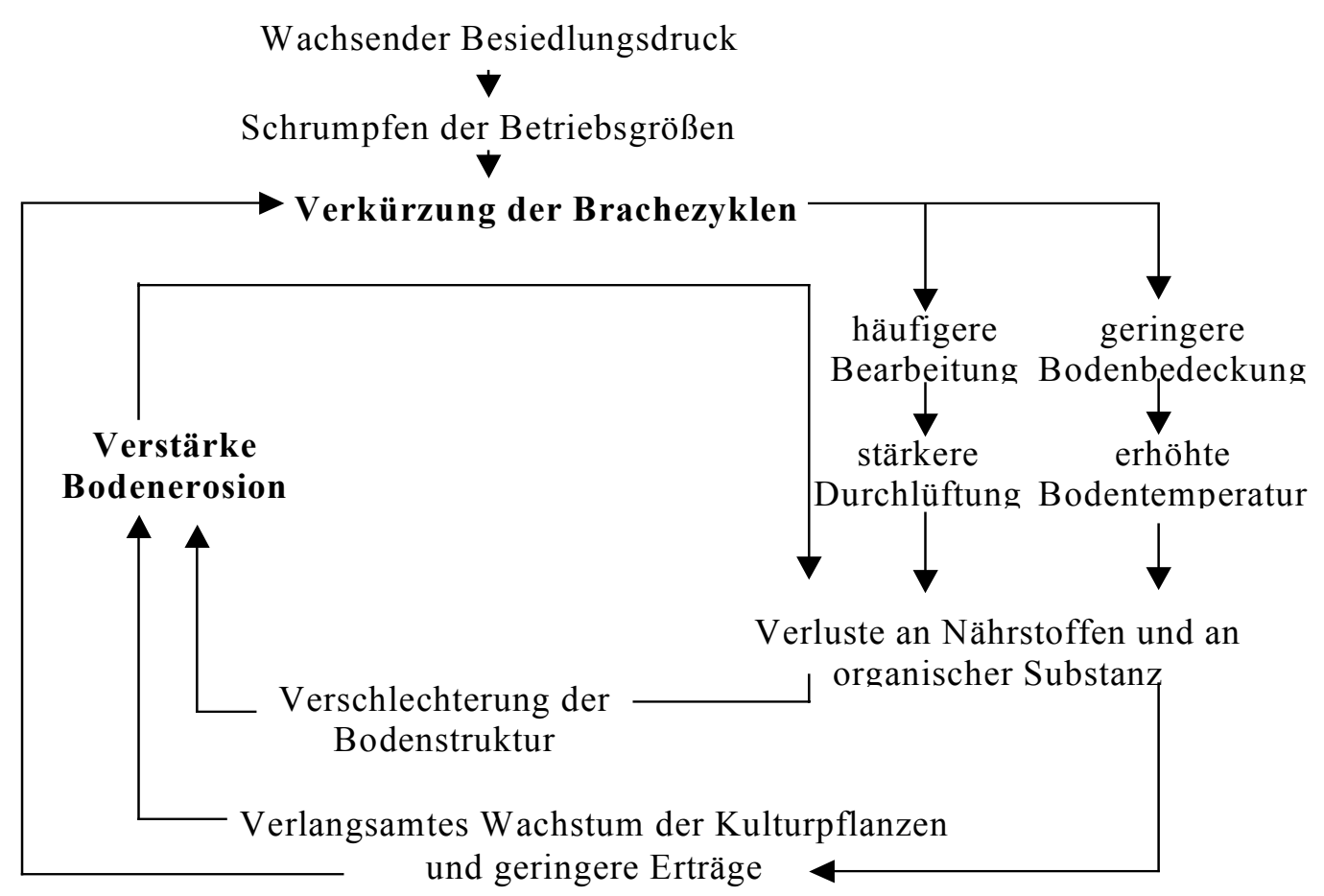

Abb.49: Fortschreiten von Bodendegradation und -erosion

Der ständige Verlust landwirtschaftlicher Nutzfläche durch Bodendegradation und erosion und das starke Bevölkerungswachstum machen die Erschließung von Neuland, wo immer dies noch möglich ist, unumgänglich. Dabei bleibt - neben der Trockenlegung der Talsümpfe oder der Umsiedlung von Bauern in bislang von der Besiedlung ausgesparten Räumen- nur ein Ausweichen auf immer steilere Hänge von bis zu $45^{\circ}$ Neigung. Bereits die einmal pro Saison durchgeführte traditionelle Bodenbearbeitung kann an derart steil geneigten Hängen Bodenverluste in der Größenordnung von hundert Tonnen pro Hektar und Jahr verursachen (ERGER, 1995). Der durch Oberflächenabfluss und Bodenbearbeitung hervorgerufene Bodenabtrag kann auf solchen Standorten zum Verlust des gesamten fruchtbaren Oberbodens innerhalb eines Jahrzehnts führen.

Letzte Möglichkeit zur Neulandgewinnung ist die Rodung der Bergnebelwälder. Eine geschlossene Waldvegetation bietet hier zunächst auch an Steilhängen einen praktisch vollständigen Schutz der Böden vor Erosion, die Abtragsraten erreichen nur Bruchteile einer Tonne pro Hektar und Jahr. Die Rodung der an die Standortbedingungen optimal angepassten Vegetation und ihre Ersatz durch saisonale Baumarten lässt den Bodenabtrag auch in nur wenigen Anbaujahren häufig auf hundert Tonnen pro Hektar und Jahr und mehr, also um vier Zehnerpotenzen, ansteigen. 
Die meisten Kleinbauern sind sich des Problems nachlassender Bodenfruchtbarkeit durchaus bewusst, da die Bodendegradation auf vielen Standorten mit großer Geschwindigkeit voranschreitet, dass sie sich innerhalb weniger Jahre in einer deutlichen Abnahme der Erträge bemerkbar macht.

Die Zunahme des Bevölkerungswachstum und der Wunsch der Bauern, ihren Lebensstandard $\mathrm{zu}$ erhöhen, indem sie von der Selbstversorgung $\mathrm{zu}$ einer marktorientierten Produktion übergehen, setzen das Land unter Druck, was zu einer Verkürzung und manchmal zur Aufgabe von Brachezeiten führt. Andere Verfahren als Wanderfeldbau sind erforderlich, um diese Probleme zu lösen. Solche Verfahren schließen Zwischenfruchtanbau, Mulchen und Agroforstsystem ein. Der Schutz der Böden vor Erosion ist ein wichtiges Thema der Beratungsdienste, aber die meisten Bauern halten dies für eine unnötige Arbeitslast ohne wirklichen Wert.

\subsubsection{Bodenerosion und Pflanzenertrag}

Die Bodenfruchtbarkeit wird von einer großen Zahl von Bodeneigenschaften bestimmt, die über die Ertragsleistung eines Standortes entscheiden. Durch die Prozesse Abtrag und Auftrag werden wichtige Bodeneigenschaften verändert, wie in Kap. 6.3 \& 6.5 .2 dargestellt. In diesem Kapitel wird der Ertrag von traditionellem Wanderfeldbau und Agroforst mit Mulch verglichen. Desweiteren wird untersucht, welche Parameter den Ertrag bestimmen.

In Abb. 50 ist die Wachstumsveränderung von Mais durch Bodenerosion dargestellt: Wenn die Maispflanzen 3-4 Blätter haben, ist die Wuchshöhe von $20 \mathrm{~cm}$ noch gleich auf beiden Testparzellen (1. und 4. Jahr nach Brandrodung). 


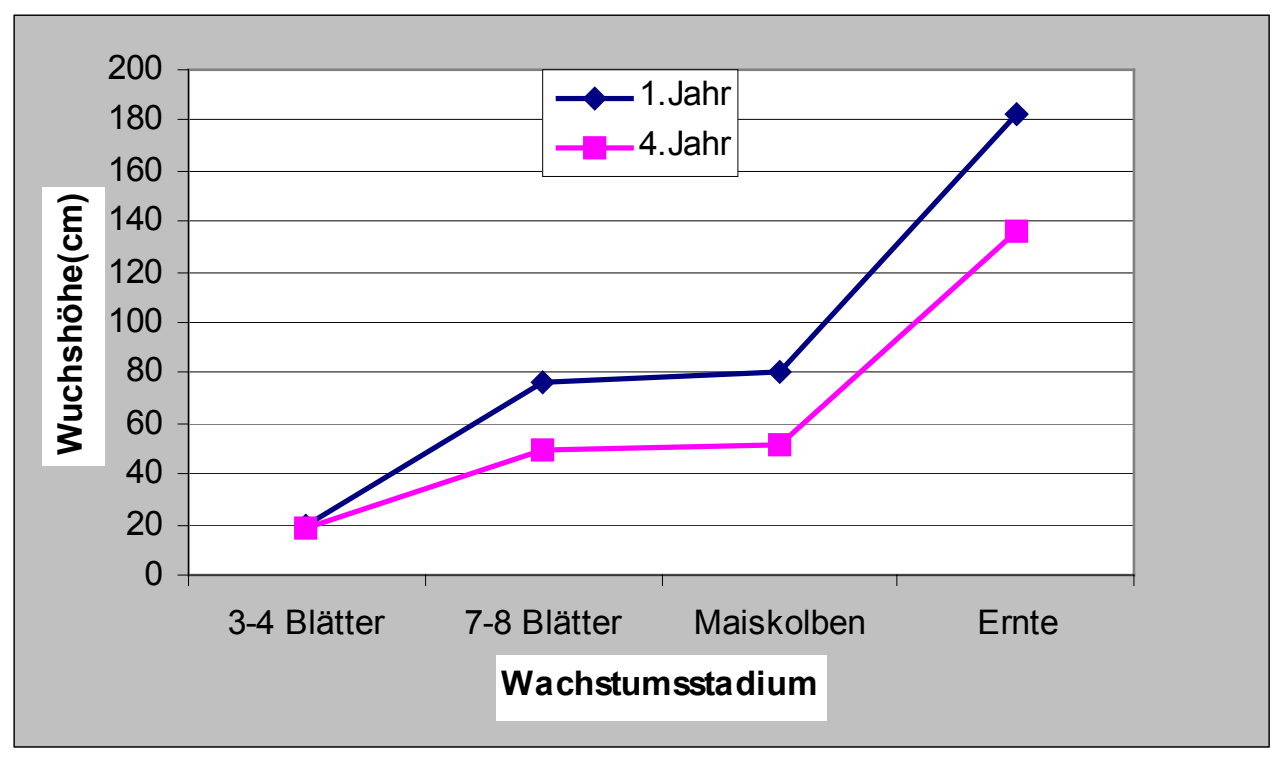

\section{Abb. 50: Wachstum von Mais im 1. und 4. Jahr nach Brandrodung (Testparzelle Dialinh, Babe)}

Danach besteht ein großer Unterschied in der Wuchshöhe der Maispflanzen. Im ersten Jahr nach Brandrodung war der Mais 1,84 m groß, während er im vierten Jahr nur 1,34 m hoch war. Die Gründe dafür sind, dass im vierten Jahr nach Brandrodung der Boden sehr viel ärmer an Nährstoffen aufgrund des Verlustes durch Bodenabtrag ist.

MÜLLER-SÄMANN (1996) folgert daraus, dass einsaisonale Intensivbrachen das Ertragsniveau von Böden stabilisieren, aber nicht erhöhen können, und dies auch nur dann, wenn sie mit geeigneten Kulturen alternieren. Das Verhältnis von Kultur- und Brachezeit sollte maximal bei 2:1 liegen, um einen langfristigen Ausgleich der Ertragsbilanz zu erreichen. Der Autor konnte nachweisen, dass die Erntebilanz einer Maiskultur in 22 Jahren bei 14 Kulturperioden und acht Bracheperioden (einsaisonale Brache mit Mucuna utilis oder Crotalaria juncea) doppelt so hoch war wie bei 22jähriger reiner Maiskultur (WEBSTER \& WILSON, 1966).

Viele Ferralsole in Südostasien dienen dem Anbau von Mais, Kaffee, und Kakao. Die Erträge von Ackerfrüchten sinken aber bereits im dritten Jahr stark ab, weil die an Humus gebundenen Nährstoffe nach Brandrodung und intensivem Humusabbau rasch aus dem Oberboden ausgewaschen werden, was auf $\mathrm{zu}$ geringe Transpirationsleistung der Ackerfrüchte und zu schwache Nährstoffbindungsvermögen der Bodenminerale zurückgeht (SCHEFFER/ SCHACHTSCHABEL, 1998).

GEROLD (1991b) führt den Ertragsrückgang auf den 4-5- jährigen brandgerodeten Anbauparzellen im immergrünen Tieflandregenwald (Bolivien) auf verschlechterte biologisch-bodenchemische Eigenschaften zurück. 
Tab.29: Kenndaten zum Maisertrag und tatsächlicher Maisertrag

\begin{tabular}{|l|l|l|l|l|l|l|l|}
\hline Test & $\begin{array}{l}\text { Länge } \\
\text { der } \\
\text { Mais- } \\
\text { kolben } \\
(\mathrm{cm})\end{array}$ & $\begin{array}{l}\text { Durch- } \\
\text { messer } \\
\text { der } \\
\text { Mais- } \\
\text { kolben } \\
(\mathrm{cm})\end{array}$ & $\begin{array}{l}\text { Zahl der } \\
\text { Mais- } \\
\text { körner } \\
\text { pro } \\
\text { Reihe }\end{array}$ & $\begin{array}{l}\text { Zahl der Korn } \\
\text { Reihen/ } \\
\text { Maiskolben }\end{array}$ & $\begin{array}{l}\text { 1000 } \\
\text { Korn- } \\
\text { gewicht } \\
(\mathrm{g})\end{array}$ & $\begin{array}{l}\text { Mais- } \\
\text { pflanzen } \\
\text { pro m }\end{array}$ & $\begin{array}{l}\text { Ertrag } \\
\text { (kg/ } \\
\text { ha) }\end{array}$ \\
\hline 1. Jahr & 19,00 & 4,45 & 30,60 & 14,20 & 255,00 & 4,40 & 3265 \\
\hline 4. Jahr & 11,02 & 3,20 & 18,20 & 11,00 & 250,00 & 3,90 & 962 \\
\hline
\end{tabular}

Der Ertrag von Pflanzen ist ein Indikator der Bodenfruchtbarkeit. Wenn die Bodenfurchtbarkeit hoch ist, ist der Ertrag von Pflanzen auch erhöht. Aus Tab.29 kann man entnehmen, dass der Ertrag von Mais im ersten Jahr nach Brandrodung (3265 kg/ha) 3x höher war, als der Maisertrag im vierten Jahr (962 kg/ha). Der Grund liegt darin, dass der Boden im vierten Jahr nach Brandrodung sehr am an Nährstoffen ist. Aufgrund sehr starker Bodenerosion verliert der Boden die für das Pflanzenwachstum notwendigen Nährstoffe.

Die Bodenerosion würde also nicht nur die Produktionskosten erhöhen, indem mit einem Mehrbedarf an Mineraldünger und Kraftstoff zu rechnen ist, sie würde ebenfalls die Ernteerträge verringern. Bei Mais würde ein Übergang von leichter zu mäßiger Erosion die durchschnittlichen Erträge bereits um etwa 16 t/ha verringern, und beim Übergang zur 3. Kategorie mit schwerer Erosion käme ein Verlust von weiteren $7 \mathrm{t} / \mathrm{ha}$ hinzu (BROWN \& WOLF, 1985).

Die Flächenerträge von Kulturpflanzen im Agroforstsystem \& Mulch sind gegenüber traditionellem Maisanbau um 18,8\% erhöht, wobei die Pflanzendichte der Kulturen in beiden Fällen etwa gleich ist. Dabei wird deutlich, dass eine Düngung mit Tephrosia candida zu einer Erhöhung des Kornertrags pro Pflanze führt. Eine wichtige Rolle spielt auch die Verbesserung des Saatgutes, erkennbar an einem deutlich erhöhtem Tausendkorngewicht.

In semi-humiden tropischen Gebieten sind Nebenwirkungen von Mulch in Form niedrigerer Bodentemperaturen und erhöhter Bodenfeuchte nützlich und können zu Ertragssteigerungen beitragen. In vielen Arbeiten wird der Grund des Produktionsrückganges kontrovers diskutiert. Dass die Pflanzenproduktion nach der Inkulturnahme von tropischen Böden schnell absinkt, wird im allgemeinen auf den 
Nährstoffentzug durch die Anbausorten, die Bodendegradation - Erosion sowie das Einfallen von Unkräutern zurückgeführt (SANCHEZ, 1976; GREENLAND \& LAL, 1977; SCHULTZ, 1995).

Der Boden dient dem Menschen u.a. als Produktionsgrundlage für Nahrungs- und Futtermittel und pflanzliche Rohstoffe. Ein Kriterium für die Nachhaltigkeit der landwirtschaftlichen Nutzung von Böden ist die Erhaltung der Produktivität. Ertragsveränderungen infolge Erosion genau zu quantifizieren ist von erheblicher Bedeutung, da nur dann dem Landwirt die Notwendigkeit von Erosionsschutzmaßnahmen offenbar wird.

\subsubsection{Art und Grad der Bodenbedeckung}

Die Vegetationsbedeckung schützt in Abhängigkeit von ihrem Entwicklungszustand den Boden vor aufprallendem Regen, da sie die Fallgeschwindigkeit der Tropfen abpuffert und damit insbesondere die splash- Wirkung (Planschwirkung) der Regentropfen abschwächt. Je länger und flächendeckender der Boden von der schützenden Vegetation eingenommen wird, desto geringer ist die Erosionsgefahr. Die Erosionsanfälligkeit der Fläche ist je nach Testfläche somit deutlich unterschiedlich.

In der Tab.30 werden Flächen verschiedener Gesamtdeckungsgrade im Hinblick auf Erosionserscheinungen miteinander verglichen.

Tab. 30: Trockenmasse von Bodenbedeckungspflanzen der Testparzelle im Dialinh, Babe 2001

\begin{tabular}{|c|c|c|c|c|c|c|c|c|}
\hline $\begin{array}{c}\text { Test- } \\
\text { par- } \\
\text { zelle }\end{array}$ & \multicolumn{2}{|c|}{ 1.Jahr } & \multicolumn{2}{|c|}{ 4.Jahr } & \multicolumn{2}{|c|}{ Brache } & \multicolumn{2}{|c|}{$\begin{array}{l}\text { Agroforst. } \\
\text { mit Mulch }\end{array}$} \\
\hline $\begin{array}{l}\text { Mo- } \\
\text { nate }\end{array}$ & $\begin{array}{l}\text { Un- } \\
\text { kraut } \\
(\mathrm{g} / \mathrm{m} 2)\end{array}$ & $\begin{array}{l}\text { Boden- } \\
\text { Bedeck- } \\
\text { ung } \\
(\%)\end{array}$ & $\begin{array}{l}\text { Un- } \\
\text { kraut } \\
(\mathrm{g} / \mathrm{m} 2)\end{array}$ & $\begin{array}{l}\text { Boden- } \\
\text { Bedeck- } \\
\text { ung } \\
(\%)\end{array}$ & $\begin{array}{l}\text { Un- } \\
\text { kraut } \\
(* *) \\
(\mathrm{g} / \mathrm{m} 2)\end{array}$ & $\begin{array}{l}\text { Boden } \\
\text { Bedeck- } \\
\text { ung } \\
(\%)\end{array}$ & $\begin{array}{l}\text { Un- } \\
\text { kraut } \\
(*) \\
\left(\mathrm{g} / \mathrm{m}^{2}\right)\end{array}$ & $\begin{array}{l}\text { Boden- } \\
\text { Bedeck- } \\
\text { ung } \\
(\%)\end{array}$ \\
\hline April & 0 & 0 & 0 & 0 & 2657 & 75 & 251 & 14,5 \\
\hline Juni & 216 & 65 & 176 & 50 & 2842 & 95 & 525 & 80 \\
\hline $\begin{array}{l}\text { Okto- } \\
\text { ber }\end{array}$ & 117 & 60 & 70 & 35 & 2745 & 90 & 334 & 60 \\
\hline
\end{tabular}


Der Bedeckungsgrad der einzelnen Parzellen zu Versuchsbeginn lag unterschiedlich hoch:

Die Parzellen Brache und Agroforst mit Mulch wiesen zu Anfang der beiden Versuche einen hohen Bedeckungsgrad von 75\% und 14,5\% auf. Danach ist in Brache die Bodenbedeckung am größten, weil diese Parzelle schon 4 Jahre lang brach lag und einzelne Gehölze mit einem Durchmesser von 5-8 cm und Lianen (Schlingpflanzen) gewachsen sind. Deswegen erreicht der Bodenbedeckungsgrad im Juni 95\%.

Darüber hinaus (s. Tab. 30) haben sich Kulturpflanzen und Erosionsschutzpflanzen auf den Erosionsmessparzellen unterschiedlich schnell entwickelt haben.

Am Anfang der Regenzeit sind die Böden in 1.Jahr und 4.Jahr nicht von Pflanzen bedeckt. Die Maispflanzen sind gerade erst gesät. Deswegen ist der Boden überhaupt nicht geschützt. Das Pflanzenwachstum folgt einem ähnlichen Muster, wie die Zunahme des Niederschlages, erreicht seinen Höhepunkt jedoch später als der Niederschlag. Die erosionsanfälligste Zeit liegt am Anfang der Regenzeit, wenn die Niederschlagsmenge hoch ist, aber die Pflanzendecke noch nicht dicht genug ist, um den Boden ausreichend zu schützen. Daher geht der Erosionshöhepunkt dem Niederschlagshöhepunkt voran.

Die Maispflanzen bewirkten 4 Wochen nach der Einsaat eine 10\%ige Bodenbedeckung von 1.Jahr und 4.Jahr. Danach besteht im Juni ein Unterschied zwischen 1.Jahr und 4.Jahr, auf Parzelle 1.Jahr wird eine Trockenmasse von $216 \mathrm{~g} / \mathrm{m}^{2}$ erreicht, auf Parzelle 4.Jahr erreicht die Trockenmasse nur $176 \mathrm{~g} / \mathrm{m}^{2}$. Im Oktober reduziert sich der Bodenbedeckungsgrad, weil in diese Zeitraum Mais sowohl auf Parzelle 1.Jahr als auch auf Parzelle 4.Jahr geerntet wurde und die Trockenzeit langsam angefangen hat. Die Entfernung der Vegetation führte zu einer Zunahme von Oberflächenabfluss und Erosion.

Die Entwicklung sowie die Zunahme der Bodenbedeckung scheint aber auch vom Agrarsystem abhängig zu sein. In Parzelle Agroforstsystem mit Mulch stieg der Bodenbedeckungsgrad von 14,5\% im April auf 80\% im Juni. Die gute Entwicklung von Tephrosia candida als Heckenreihe bietet einen hohen Bewuchs als Bodenbedeckung. Young (1991a), Thai PHien (1998), Minh Ha HoAng FAgerström, (2000) fanden, dass diese Pflanzen sowohl als Gründüngung, als auch als Bodendecker für eine ökologische Nachhaltigkeitslandwirtschaft geeignet ist.

- Eine schnelle Bodenbedeckung in relativ kurzer Zeit, verhindert Oberflächenabfluss und schützt den Boden vor Abtrag. 
- Der Erhalt der Nährstoffe reduziert sich nicht mit der Tiefe des Bodenprofils und die hohen Nährstoffgehalte, besonders Stickstoff (200-300 kg N/ha) und Kalium (300$500 \mathrm{~kg} / \mathrm{ha}$ ), verhindern eine Phosphatfixierung und ein hohes Nährstoffaufschlussvermögen.

- Die Kationenaustauchkapazität verbessert sich.

- Die Pflanzen haben eine hohe Affinität zu Mykorrhiza, um das Inokulum im Boden zu verbessern.

- Textur und Wasserspeichervermögen der Böden verbessert sich.

- Die Gründüngungspflanze erlaubt in ihrer Nebenfunktion auch eine Nutzung als Futterpflanze.

Vegetation wirkt wie eine Schutzschicht oder ein Puffer zwischen der Atmosphäre und dem Boden. Die oberirdischen Bestandteile wie Blätter und Stengel absorbieren einen Teil der Regenenergie, des fließenden Wassers und des Windes, so dass weniger davon den Boden trifft. Die unterirdischen Pflanzenteile, das Wurzelsystem, tragen zur mechanischen Bodenfestigkeit bei.

\subsubsection{Nährstoffverlust}

„Jeder Bodenabtrag bedeutet also Substanzverlust und damit eine Schädigung des Bodens und dessen Fruchtbarkeit. Bodenerosion sollte deshalb auch möglichst vermieden werden“"

Je größer der Bodenabtrag, desto rascher verliert der Boden seine Fruchtbarkeit und seine ökologischen Funktionen.

Böden stellen offene Systeme dar. So unterliegen sie einerseits einer Stoffzufuhr (Atmosphäre, Dünger), andererseits werden Stoffe aus den Böden in andere Ökosysteme eingetragen. In erosionsbeeinflussten Bodenlandschaften werden Nährstoffe in gelöster Form oder partikulär gebunden abgetragen (AUERWALD, 1985; ROHRER, 1985). Bestimmte Nährstoffe wie Stickstoff und Phosphor sind aufgrund ihrer umweltrelevanten Wirkung (Eutrophierung von Oberflächengewässern, Qualitätsprobleme bei Grundwasser für Trinkwasserzwecke) von besonderer Bedeutung. Zur Beurteilung von Umweltbelastungen und Bodenfruchtbarkeit mittels Nährstoffverluste wurden die Bodenabträge der einzelnen Parzellen verwendet.

Aus der Analyse des Sediments wurde der Gesamtverlust einzelner Bodennährstoffe und Schwermetalle ermittelt wie in Tab.31 aufgezeigt. 
Bodenerosion ist der Hauptfaktor des Verlustes an Nährstoffen, insbesondere von Kationen. Der Nährstoffverlust hängt von der Art der Bodenbearbeitung und den angebauten Kulturpflanzen ab. Der $\mathrm{pH}$-Wert sank mit fortschreitender Bewirtschaftungsdauer von 6,2 (KCl) im ersten Jahr nach der Brandrodung auf 4,4 im vierten Jahr nach Brandrodung. Die KAKeff sank auf einen Wert $<7$ (cmol/kg Boden) auf Parzellen auf denen Mais angebaut wurde, was die rasche Degradierung der Böden belegt. Die Basensättigung verringerte sich auf den bewirtschafteten Zellen auf $<50 \%$. Besonders drastisch war ihre Verringerung auf der Parzelle mit Alang-Gras, wo sie nur noch $32 \%$ betrug.

Tab.31: Nährstoffverlust durch Bodenerosion

\begin{tabular}{|c|c|c|c|c|c|}
\hline \multirow{2}{*}{\multicolumn{2}{|c|}{ Bodenabtrag(kg/ha) }} & 1. Jahr & 4.Jahr & Brache & $\begin{array}{l}\text { Agroforst } \\
\text { mit Mulch }\end{array}$ \\
\hline & & 43042 & 58270 & 2216 & 3686 \\
\hline \multirow{19}{*}{$\begin{array}{l}\text { Nährstoff- } \\
\text { verlust } \\
\text { (kg/ha/a) }\end{array}$} & $\mathrm{C}$ & 1234,01 & 969,03 & 90,88 & 116,22 \\
\hline & $\mathrm{N}$ & 82,21 & 73,42 & 6,43 & 6,78 \\
\hline & $P$ & 18,99 & 23,03 & 1,10 & 1,98 \\
\hline & $\mathrm{K}$ & 854,58 & 1468,02 & 50,58 & 54,76 \\
\hline & $\mathrm{Ca}$ & 89,74 & 97,81 & 8,14 & 18,84 \\
\hline & $\mathrm{Mg}$ & 642,8 & 1035,24 & 29,82 & 17,57 \\
\hline & $\mathrm{Na}$ & 22,65 & 26,25 & 3,75 & 4,86 \\
\hline & $\mathrm{Fe}$ & 1864,56 & 2568,22 & 104,48 & 186,54 \\
\hline & $\mathrm{Al}$ & 3716,72 & 5171,25 & 220,03 & 377,44 \\
\hline & $\mathrm{Mn}$ & 28,28 & 35,81 & 1,23 & 1,65 \\
\hline & Mo & 0,06 & 0,05 & 0,00 & 0,01 \\
\hline & $\mathrm{Ni}$ & 1,83 & 2,91 & 0,12 & 0,20 \\
\hline & $\mathrm{Pb}$ & 14,91 & 21,80 & 0,08 & 0,14 \\
\hline & $\mathrm{S}$ & 10,48 & 11,13 & 0,78 & 1,06 \\
\hline & $\mathrm{Ti}$ & 0,11 & 0,10 & 0,45 & 0,02 \\
\hline & $\mathrm{Zn}$ & 6,37 & 7,75 & 0,34 & 0,55 \\
\hline & Co & 0,16 & 0,29 & 0,01 & 0,00 \\
\hline & $\mathrm{Cr}$ & 4,17 & 5,70 & 0,24 & 0,36 \\
\hline & $\mathrm{Cu}$ & 0,84 & 1,36 & 0,07 & 0,09 \\
\hline \multicolumn{2}{|c|}{ Maisertrag (kg/ha) } & 3265 & 962 & - & - \\
\hline
\end{tabular}


Es konnte festgestellt werden (s.Tab. 31), dass der Pflanzenertrag, als Indikator für Bodenfruchtbarkeit, in enger Korrelation steht mit dem Bodenabtrag und dem gemessenen Nährstoffverlust von 73 - $82 \mathrm{~kg}$ Stickstoff ha/a, 19-23 kg Phosphor ha/a und 854-1468 kg Kalium ha/a.

Je höher der Oberflächenabfluss, desto höher die Nährstoffverluste. Die Menge des Nährstoffverlustes steigt in der Reihenfolge: $\mathrm{Al}>\mathrm{Fe}>\mathrm{C}>\mathrm{K}>\mathrm{Mg}>\mathrm{Ca}>\mathrm{N}>\mathrm{Na}>\mathrm{Mn}$ $>\mathrm{P}>\mathrm{Pb}>\mathrm{Zn}>\mathrm{S}>\mathrm{Ti}>\mathrm{Mo}$. Diese Reihe variiert zu dem Ergebnis, das in der Provinz Vinh phu im Bergland von Vietnam auf einem Boden aus Tonschiefer, bei einer Hangneigung von $10-30^{\circ}$ und unter Maniok- und Sojabohnenanbau ermittelt wurde. Dort sanken die Nährstoffverluste in der Reihenfolge $\mathrm{Ca}>\mathrm{Fe}>\mathrm{Al}>\mathrm{K}>\mathrm{C}>\mathrm{Mg}>\mathrm{N}>\mathrm{P}$. Auch dort konnte festgestellt werden, dass Bodenerosion die Hauptursache für die Verringerung an Nährstoffen ist. Infolge des Abtrages organischer Substanz vermindert sich der Anteil an verfügbarem Phosphors um 90\%, der verfügbare Stickstoffs um $80 \%$.

Es findet kein Ersatz der Nährstoffverluste über Düngemittel statt. Tierischer Dung und Mineraldünger werden in der traditionellen Landwirtschaft nur für Nassreis aber nicht für die Bergkulturen (Brandrodungsflächen) verwendet. Im 4. Jahr nach Brandrodung ist der Maisertrag auf ein Drittel des Ausgangswertes gesunken, so dass im darauf folgendem Jahr die Fläche aus der Nutzung genommen werden muß (Brache).

Diese Ergebnisse stimmen mit den Ergebnissen von LE VAN TIEM (1994) überein. Er hat festgestellt, dass Mangel an Kalium und Phosphor im Bergland von Vietnam häufig sind. Er schlägt die mangelnde Phosphor- und Kaliumverfügbarkeit als Indikator für Bodendegradation im Bergland von Vietnam vor. Feldversuche in der Hoabinh-Provinz, (nordvietnamesisches Bergland) haben herausgefunden, dass sich im 3. Jahr nach der Rodung der Trockenreisertrag schnell verringert. Er beträgt dann nur noch $37 \%$ des Trockenreisertrages des ersten Jahres unmittelbar nach der Brandrodung (VIEN, 1996).

Verursacht wird die starke Abnahme des Ertrages durch Nährstoffverluste infolge Bodenerosion. Im Kapital 6.3.2 wird erklärt, dass ein deutlicher Verlust an organischer Substanz und Stickstoff stattfindet. Mit dem Verlust an organischer Substanz und der damit verbundenen Verringerung der Austauschkapazität versauern die Böden.

In vielen Untersuchungen über Bodenerosion in Vietnam wurden Menge und Zusammensetzung der Abtragssedimente analysiert. Bei einem Bodensediment mit den mengenmäßigen Anteilen $\mathrm{C}$ von $1 \%$; $: 0,1 \% ; \mathrm{P}_{2} 0_{5}: 0,08 \% ; \mathrm{K}_{2} 0_{5}: 0,06 \%$ und einem ermitteltem durchschnittlichen Bodenabtrag von 10 t/a stellte SIEM \& PHIEN (1999) folgende Rechnung auf. Um die Pflanzenernährung zu gewährleisten, entspräche das 
einem Düngemitteleinsatz von: 0,5 t Stalldünger, $20 \mathrm{~kg}$ Urea (Harnstoff), $44 \mathrm{~kg}$ SuperPhosphat, $10 \mathrm{~kg} \mathrm{~K}_{2} \mathrm{~S}_{4}$ und damit verbundenen Kosten von $150000 \mathrm{VND} / \mathrm{ha}$ (10 USD). Auf die gesamte landwirtschaftlich bearbeitete Fläche Vietnams, also auf 5 Mio ha umgerechnet, bedeutet das bei einem durchschnittlichen Bodenabtrag von $10 \mathrm{t} / \mathrm{ha}$ einen Kostenaufwand von 7500 Million VND/Jahr (50 Millionen USD/Jahr), um die Nährstoffverluste durch Düngemittel wieder auszugleichen wie die Ergebnisse in Kap. 6.4 der Bodenerosionsmessung belegen. In Tab. 32 wurde der Kostenaufwand an NPKDüngung für die in dieser Arbeit untersuchten Parzellen berechnet.

Tab. 32: Kostenaufwand an NPK-Düngung zum Ausgleich von Nährstoffverlusten durch Bodenerosion

\begin{tabular}{|c|c|c|c|c|c|}
\hline \multicolumn{2}{|c|}{ Parzelle } & $\begin{array}{r}\text { Nährstoff- } \\
\text { verlust } \\
(\mathrm{kg} / \mathrm{ha} / \mathrm{a})\end{array}$ & $\begin{array}{l}\text { Düngung } \\
\text { (kg/ha/a) }\end{array}$ & $\begin{array}{r}\text { Gesamtverlust/ } \\
\text { ha/a (VND) }\end{array}$ & $\begin{array}{r}\text { Gesamtkosten } \\
\text { NPK/ha/a } \\
(V N D)\end{array}$ \\
\hline \multirow{3}{*}{ 1.Jahr } & $\mathrm{N}$ & 73,4 & $159,6^{*}$ & 558.600 & \multirow{3}{*}{$\begin{array}{l}5.383 .000 \\
(359 U S \$)^{1}\end{array}$} \\
\hline & $\mathrm{P}$ & 19,0 & $90,5 * *$ & 135.800 & \\
\hline & $\mathrm{K}$ & 854,0 & $1674,5 * * *$ & 4.688 .600 & \\
\hline \multirow{3}{*}{ 4.Jahr } & $\mathrm{N}$ & 82,2 & $178,7 *$ & 625.500 & \multirow{3}{*}{$\begin{array}{l}8.849 .400 \\
(590 \text { US\$) }\end{array}$} \\
\hline & $\mathrm{P}$ & 23,0 & $109,5 * *$ & 164.300 & \\
\hline & $\mathrm{K}$ & 1468,0 & $2878,4 * * *$ & 8.059 .600 & \\
\hline \multirow{3}{*}{$\begin{array}{l}\text { Agro. } \\
\mathrm{M}\end{array}$} & $\mathrm{N}$ & 6,8 & $14,8^{*}$ & 51.800 & \multirow{3}{*}{$\begin{array}{r}370.300 \\
(24,7 \text { US\$) }\end{array}$} \\
\hline & $\mathrm{P}$ & 2,0 & $9,4 * *$ & 14.100 & \\
\hline & $\mathrm{K}$ & 54,8 & $107,3 * * *$ & 304.400 & \\
\hline
\end{tabular}

Man kann feststellen, dass der Nährstoffverlust durch Bodenerosion im Brandrodungsfeldbau erheblich größer ist als im Agroforstsystem. Die notwendigen Kosten für Düngung zum Ausgleich der Nährstoffverluste betragen im Brandrodungsfeldbau das 3 - 4fache des Jahreseinkommens der Tay-Familien (s. Tab. 24). Darüber hinaus ist auch der Nährstoffentzug durch die Pflanzen zu berücksichtigen wie in Tab. 33 für Mais dargestellt. Im Untersuchungsgebiet wird aber traditionell keine Düngung auf Bergfeldern benutzt, so dass der Boden sehr schnell degradiert. 
Tab.33 : Richtwerte für Nährstoff - Bedarf von Mais (nach PAgEL 1982)

\begin{tabular}{|l|l|l|l|}
\hline $\begin{array}{l}\text { Nährstoff } \\
(\mathrm{kg} / \mathrm{ha})\end{array}$ & niedriger Ertrag & mittlerer Ertrag & hoher Ertrag \\
\hline $\mathrm{N}$ & $30-50$ & $60-120$ & $150-250$ \\
\hline $\mathrm{P}$ & $6-12$ & $20-30$ & $40-50$ \\
\hline $\mathrm{K}$ & $20-40$ & $60-110$ & $130-170$ \\
\hline $\mathrm{Mg}$ & $4-8$ & $10-16$ & $20-30$ \\
\hline
\end{tabular}

\subsubsection{Schussfolgerung:}

In den vorangegangenen Kapiteln wurde festgestellt, dass

1. Bodenerosion findet im Bergland von Vietnam auf landwirtschaftlich genutzen Hängen vor allem in Folge hoher und konzentrierter Niederschläge während der Regenzeit von April bis September statt. Bei einer Regenintensität 0,15-0,30 $\mathrm{mm} / \mathrm{Min}$. finden bei einer durchschnittlichen Hangneigung von $22^{\circ}$ starker Oberflächenabfluss und Bodenerosion statt. Die Erosion zu Beginn der Regenzeit ist stärker als am Ende der Regenzeit.

2. Im Brandrodungsfeldbau gingen durch Abtrag während der Regenzeit zwischen $43 \mathrm{t} / \mathrm{ha}$ und $58 \mathrm{t} / \mathrm{ha} / \mathrm{a}$ Boden verloren. Der Bodenabtrag auf Brache oder Agroforstsystem mit Mulch betrug nur 3, $7 \mathrm{t} / \mathrm{ha} / \mathrm{a}$.

3. Die Vegetationsdecke modifiziert die Wirkung der Hangneigung. Es wurde festgestellt, dass bei Deckungsgraden von etwa $60 \%$ oder weniger die Schutzwirkung der Vegetation für den Boden nahezu aufgehoben ist. Der Zustand der Vegetationsdecke zu einem bestimmten Zeitpunkt gibt damit die aktuelle Erosionsgefährdung an.

4. Bodenerosion ist die Hauptursache von Bodendegradation. Als Nährstoffverluste wurden 996-1234 kg org. Substanz /ha/a, 73-82 kg N/ha/a, 854-1468 kg K/ha/a, 19$23 \mathrm{~kg} \mathrm{P} / \mathrm{ha} / \mathrm{a}, 642-1035 \mathrm{~kg} \mathrm{Mg} / \mathrm{ha} / \mathrm{a}, 89-97 \mathrm{~kg} \mathrm{Ca} / \mathrm{ha} / \mathrm{a}$ gemessen und sind als schwerwiegend einzustufen. Der Kostenaufwand an NPK zum Ausgleich von Nährstoffverlusten durch Bodenerosion beträgt im Brandrodungsfeldbau 5.383.0008.849.400 VND/ha/a und entspricht damit dem 3-4 fachen des Jahreseinkommens 
der Tay-Familien. Dagegen liegt der Kostenaufwand im Agroforst mit Mulch bei nur $370.300 \mathrm{VND} / \mathrm{ha} / \mathrm{a}$.

5. Der Maisertrag auf der Parzelle im 4. Jahr nach Brandrodung betrug nur ein Drittel der des 1. Jahres.

6. Die Anlage von Hecken aus Tephrosia candida und Leuceana glauca stellt eine sehr gute Möglichkeit zur Reduzierung des Bodenabtrages dar.

Zusammenfassend ist festzustellen, dass die Ackerflächen insgesamt als sehr erosionsanfällig einzustufen sind und die Integration von Bäumen und insbesondere von Hecken aus Tephrosia candida und Leuceana glauca eine wirkungsvolle Begrenzung von Oberflächenabfuß und Bodenabtrag darstellt. Darüber hinaus können sie unmittelbar (über die Ernährung des Viehs) sowie mittelbar (Produktion von Mulch- und Kompostmaterial) zu einer Steigerung der landwirtschaftlichen Produktivität beitragen. Schutzmaßnahmen wie Agroforstsystem mit Mulch können gut zu einer Verminderung des Bodenabtrags beitragen, werden aber im Untersuchungsgebiet kaum angewandt (Tab. 20 in Kap. 5). Es besteht die Notwendigkeit der Beratung der Bauern, um ihnen neue gezielte agrarforstwirtschaftliche Maßnahmen mit angepassten Nutzungssystem nahe zu bringen.

\subsection{Wirkung der Schutzmaßnahme gegen Erosion und Erhaltung der Bodenfruchtbarkeit:}

„A little effort to spread corn residue on the soil surface as mulch after harvest will go a along way to reduce both run off and soil loss..." (BABALOLA \& CHHEDA, 1975)

Nach LAL (1975) liegt der größte Vorteil des Agroforstsystems mit Mulch für tropische Böden in der „,sicheren und billigen Minderung der Erosion“. Er hat heraus gefunden: Durch Mulchraten von nur 2 t/ha wurden der Oberflächenabfluss um $60 \%$ und die Bodenerosion um durchschnittlich über $90 \%$ gemindert. Bei der dreifachen Menge (6 t/ha oder $600 \mathrm{~g} / \mathrm{m}^{2}$ ) konnten der Oberflächenabfluss zu 90\% und die Bodenerosion quasi völlig verhindert werden. Aus Tab. 34 ist ersichtlich, dass das Anpflanzen von Heckenreihen als Erosionschutzmaßnahme ein deutliche Verminderung des Bodenabtrags zur Folge hat. Im Babe - Distrikt konnte der Abtrag im Durchschnitt um 91 - 93\% im Vergleich zu Gründüngung ohne Heckenreihen reduziert werden. 
Tab.34: Wirkung von Gründüngungspflanzen gegen Bodenerosion

\begin{tabular}{lccr}
\hline \multirow{2}{*}{ Ort } & \multicolumn{2}{c}{ Bodenerosion (t/ha/a) } & \multicolumn{2}{c}{$\begin{array}{c}\text { Reduzierung \% } \\
\text { (verglichen mit } \\
\text { keine Hecken) }\end{array}$} \\
\cline { 2 - 4 } & $\begin{array}{c}\text { Keine Hecken mit } \\
\text { Gründüngung }\end{array}$ & $\begin{array}{c}\text { Gründüngung mit } \\
\text { Heckenreihen }\end{array}$ & 27,8 \\
\hline Quangchau (China) & 80,2 & 31,8 & 65,3 \\
\hline Kuamang (Indonesien) & 204,7 & 5,2 & 84,5 \\
\hline Mabini (Philipinen) & 60,1 & 2,0 & 91,3 \\
\hline Tanay (Philipinen) & 49,7 & 44,2 & 94,4 \\
\hline Chiangrai (Thailand) & 129,7 & 0,7 & 65,9 \\
\hline Chiangmai (Thailand) & 10,6 & 5,0 & 93,4 \\
\hline Luongson (Vietnam) & 30,0 & $\underline{\mathbf{3 , 7}}$ & $\underline{\mathbf{9 1 , 4 - 9 3 , 6}}$ \\
\hline$\underline{\text { Babe (Vietnam) }}$ & $\underline{\mathbf{4 3 - 5 8}}$ & & 83,3 \\
\hline
\end{tabular}

(Quelle: auserdem Babe, andere aus SIEM \& PHIEN, 1999)

In bereits erfolgten Untersuchungen an der Thai Nguyen Universität wurde daher versucht, traditionellen Vorbildern folgend, ausdauernde Sträucher und Bäume an Stelle der Gründüngung zu pflanzen. Der Erfolg ist eigentlich überzeugend. Die Hecken liefern das Doppelte an Schnitt, insbesondere haben sie bis zum Ende der Trockenzeit frisches Grün. Außer Laub können auch Stangen für Bohnen, Tomaten und ähnliches gewonnen werden, die Böschung schließlich ist für praktisch unbegrenzte Zeit befestigt. Inzwischen verfügt man über Erfahrung mit 10 Pflanzenarten, die sich jedem Standort und jedem Nutzungswunsch anpassen lassen. Auch gemischte Hecken sind leistungsstark, wenn eine Leguminose (Tephrocia candida oder Leucaena glauca) mit einer nicht Stickstoff sammelnden Art (Maniok, Mais, Trockenreis...) gemeinsam gepflanzt wird. Die wichtigsten Heckenpflanzen sind: Flemingea congesta, Vertiver, Tephrocia candida oder Leucaena glauca. Der Heckenschnitt ist übrigens stets vielseitig verwendbar: neben Gründungung und Mulch auch als Futter, als Holz oder Kompostmaterial.

Besonders wichtig und unverzichtbar werden Hecken, wenn von der geneigten Terrasse zu völlig ebenen oder gar sanft gegen den Hang geneigten übergegangen werden soll.

Ein als Alley-croping bezeichnetes Gründüngungssystem hat in diesem Zusammenhang mehr Interesse von seiten der Wissenschaftler erfahren als jede andere vergleichbare Gründüngungsmethode im Agroforstsystem. Diese auch „hedgerow intercroping“ genannte Technik sieht den Anbau von Feldfrüchten zwischen Hecken oder Baumreihen von stickstofffixierenden Gehölzpflanzen vor. Diese werden regelmäßig beschnitten, um 
die Beschattung der Nahrungsmittelkulturen zu minimieren. Das Schnittmaterial wird in den Gängen eingearbeitet oder als Mulch verwendet. Schnittmaterial aus Hecken, Bäume, Ernterückstände aus Kulturpflanzen und Unkräuter sind die Quellen von Mulchmaterial. Der Mulch kann zwischen die Reihen, in die Reihen oder als deckende Lage verabreicht werden.

Mulch wirkt also in zweierlei Hinsicht erosionsmindernd: Zum einen schützt er den Boden vor der Aufprallenergie der Regentropfen (dem Verschlämmen) und bewirkt dadurch, dass seine Poren zur Aufnahme der Niederschläge geöffnet bleiben. Zum anderen bremst Mulch den Abfluss des Wassers und verhindert dadurch, dass das abfließende Wasser viel Boden mittransportieren (erodieren) kann. Je größer die Erosionsgefahren durch heftige Niederschläge, je geringer die Infiltrationsfähigkeit und je erosionsanfälliger die Böden, desto eher sind Maßnahmen zum Erosionsschutz notwendig.

Das Agroforstsystem mit Mulch hat aber nicht nur eine Verminderung des Bodenabtrages, sondern dient auch der Erhaltung und Verbesserung der natürlichen Bodenfruchtbarkeit.

In den Versuchen mit jungen Strauchlinien (1.-3.Jahr) war mit zunehmender Dauer der Gründüngungsmaßnahmen im Alley-cropping-System ein Anstieg des Ertragsgewinns gegenüber der ungedüngten Reiskultur von $0 \%$ auf bis zu $20 \%$ festzustellen.

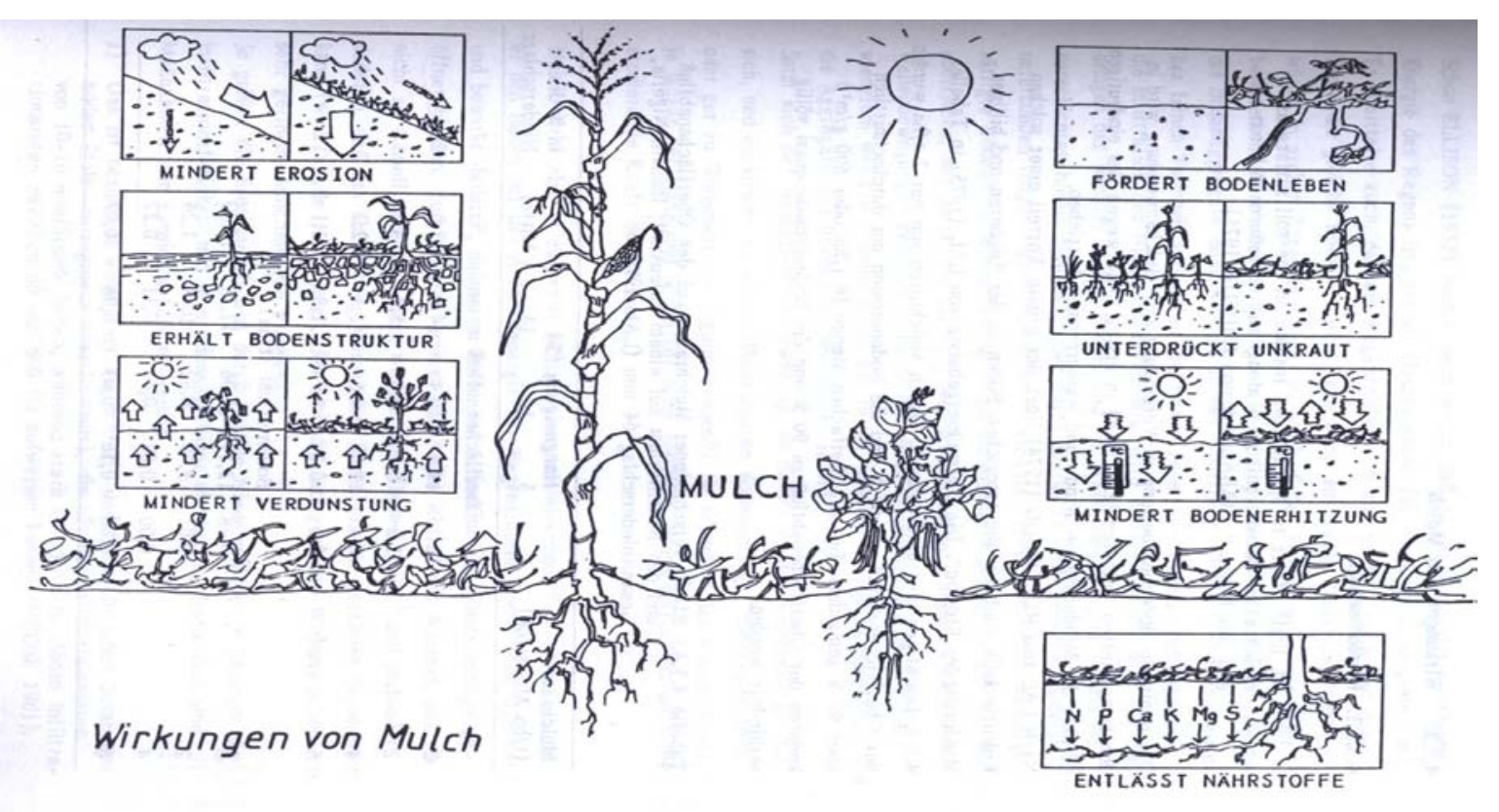

Abb.51: Wirkungen von Mulch auf die Bodenfruchtbarkeit 
Die Ertragssteigerungen waren im etablierten Alley-cropping-System (4-6.Jahr) am größten. Trotz allmählich sinkender Menge der eingebrachten Grünmasse wurde der Ertragsgewinn pro Saison auf $15-30 \%$ stabilisiert. Offentsichtlich tragen die höhenlinienparallelen Strauchlinien zur Verminderung der Erosion (Unterterrassierung) somit schon ohne direkte Düngewirkung zur Aufrechterhaltung der Bodenfruchtbarkeit bei. Weitere Nährstoffverluste durch Verbrauch, Auswaschung und Entgasung können mit dem Biomasseneintrag ausgeglichen werden. Während also das Ertragsniveau ungedüngter Reinkulturen allmählich abnimmt, kann es im Alley-cropping-System durch die Verringerung des Bodenabtrags und durch die Gründüngung mit Tephrosia- Laub trotz der offensichtlichen Konkurrenz zwischen Sträuchern und Feldkulturen stabilisiert oder sogar gesteigert werden. Der Nachfruchteffekt des Agroforstsystems mit Mulch geht in erster Linie auf den Eintrag von Stickstoff (bis $300 \mathrm{Kg} \mathrm{N} / \mathrm{ha} / \mathrm{Jahr}$ ) und Phosphor (bis 40 $\mathrm{kg} / \mathrm{ha} / \mathrm{Jahr}$ ) in leicht mineralisierbarer Form zurück. Regelmäßige Gründüngung verbesserte die Bodensituation und erhöhte die Erträge.

Die Entwicklung angepasster Techniken der Bodenfruchtbarkeitssteigerung und erhaltung mit Hilfe von Heckenreihen und anderen Gründüngungsmethoden ist seit Beginn der Arbeiten von THAI PHIEN im Jahr 1993 ein Schwerpunkt der begleitenden Forschung, welche sich möglichst nahe an der betrieblichen Praxis orientiert.

Verschiedene Gründüngungsarten erreichten ebenfalls eine hohe Biomassenproduktion (bis $9 \mathrm{t} / \mathrm{ha}$ Trockenmasse Streu und Aufwuchs nach fünf Monaten), konnten aber den Boden nicht schnell genug decken, um das Aufkommen von Unkräutern zu verhindern.

„Solange es mit den Bäumen noch Probleme gibt, lässt sich die Agroforstwirschaft zunächst auf Hecken aufbauen. Aber auch hier gilt, dass die erfolgreichsten Hecken wie Leucaena auch nur auf den guten Böden wirklich gedeihen, nur Tephrosia oder Cajanus cajan und mehrere Crotalaria-Arten bringen auch auf den schwächeren Böden überraschende Leistungen als Gründüngungs- und Heckenpflanzen. Daher wird der Beginn eines Agroforstsystems mit intensiven Heckenpflanzungen dieser Arten empfohlen“ (EGGER, 1989).

Die Gründüngung spielt in diesem System eine wichtige Rolle bei der organischen Versorgung des Bodens. Die bedeutendste Wirkung einer Leguminosengründüngung auf den Nährstoffhaushalt geht von der Stickstoffwirkung aus.

Gründüngung kann in der Regel nur die Verfügbarkeit von Stickstoff verbessern. $\mathrm{Ca}, \mathrm{Mg}$, P, auch K und Spurenelemente (B, Zn, Mo) müssen zugeführt werden (EGGER, 1995). 
Häufig wird in der Literatur auch darüber berichtet, dass mit Leguminosen-Gründüngung der Status des Bodens an verfügbaren Phosphor verbessert werden könnte (KANNT, 1983). Auf einem Standort in Niegeria konnte mit einsaisonalem Gründüngungsanbau der P-Gehalt eines Ackerbodens von 7,8 kg/ha auf 12,1 kg/ha verbessert werden. Neben solchen bodenchemischen Erklärungsansätzen zur Verfügbarkeit von Phosphaten darf nicht vergessen werden, dass durch die Verbesserung der bodenphysikalischen Bedingungen und durch die bessere N-Versorgung nach Gründüngung natürlich auch die Durchwurzelung und Wurzelbildung im Boden verbessert wird, das heißt, durch die höhere Wurzeloberfläche und durch die intensivere Durchwurzelung des Bodens in Gründüngungsfruchtfolgen wird das P-Aneignungsvermögen insgesamt verbessert. Diese Wirkung bezieht sich auch auf den Aufschluß anderer Bodennährstoffe wie $\mathrm{K}, \mathrm{Mg}$, Spurennährstoffe usw. Die Löslichkeit metallischer Spurennährstoffe wird durch Gründüngung (über Komplex- und Chelatbildung) verbessert. Die Eisen- und ManganLöslichkeit kann im Zuge der Zersetzung frischer organischer Substanz unter anaeroben Verhältnissen sehr stark ansteigen, was auf eisenreichen Böden zu Toxizität führen kann (FLAIG ET AL., 1978).

Tab.35: Nährstoff von Gründüngungspflanzen (kg/ha)

\begin{tabular}{|c|c|c|c|c|c|}
\hline Gründüngungsarten & $\mathrm{N}$ & $\mathrm{P}$ & $\mathrm{K}$ & $\mathrm{Ca}$ & $\mathrm{Mg}$ \\
\hline Tephrosia candina & 498,0 & 34,0 & 448,0 & 113,1 & 90,3 \\
Stylo & 295,3 & 25,8 & 69,0 & 115,2 & 83,0 \\
Fabaceae & 295,3 & 19,0 & 338,8 & 62,3 & 47,2 \\
Leuaceana glauca & 199,0 & 20,0 & 80,6 & 44,0 & 38,0 \\
& & & & & \\
\hline
\end{tabular}

(Quelle: SIEM \& PHIEN, 1999)

FungAMEZA (1991) konnte durch Feldversuche in der Region Kigoma/ Tansania zeigen, dass Gründüngung die Wirksamkeit von NPK- Dünger erheblich verbessert. Gründüngung kann also als ökophysiologischer Anknüpfpunkt für den Einsatz von Mineraldünger fungieren und verbessert gleichzeitig die ökonomischen Voraussetzungen, in dem sie die Abhängigkeit der Betriebe von Düngemittel zu Kauf verringert.

Gründüngung ist unter den ökonomischen Bedingungen kapitalschwacher Länder ein wichtiges Instrument zur Erhaltung der Bodenfruchtbarkeit. Die Kulturbrache ist aber immer als Ergänzung - nicht als Alternative- zu anderen Formen der organischen und mineralischen Düngung anzusehen. 
Die Gründüngungsmaßnahme hat eine ökologische Leistung. Wird nährstoff- und energiereiche Gründüngungsmasse in den Boden eingebracht, so werden verschiedene biologische und chemische Vorgänge in Gang gesetzt, die sehr komplexe Auswirkungen auf das Nährstoffangebot haben. MÜLLER-SÄMANN (1986) nennt den Stickstoffhaushalt als den wichtigsten Einflussbereich. Leguminosengründüngung weist je nach Art und Bedingungen unterschiedlich hohe und unterschiedlich schnell verfügbare Stickstoffanteile auf, die von der Nachfrucht verwertet werden können. Darüber hinaus regt die eingearbeitete Grünmasse das Bodenleben an, so dass auch stabiler HumusStickstoff mobilisiert wird. Leguminosengründüngung verbessert auch die Verfügbarkeit von Bodenphosphor. Dies ist auch auf $\mathrm{H}^{+}$-Ionen zurückzuführen, die in die Rhizosphäre abgeschieden werden und zur besseren Löslichkeit von (Roh-) Phosphaten beitragen. Eine gute Affinität der Wurzeln von Gründüngungspflanzen zu Mycorrhiza ist ebenfalls eine Eigenschaft, die zur verstärkten Anreicherung von Phosphor in der Grünmasse führen kann, da diese Pilze Phosphate im Boden mobilisieren. Die Aussagen über die Auswirkung von Gründüngung auf den Kohlenstoff- (Humus-) haushalt in tropischen Böden gehen in der Literatur stark auseinander. In Rotationen mit relativ hohem Gründüngungsanteil konnte der Humusgehalt über lange Zeit hinweg stabil gehalten werden. Je kleiner die Menge der eingearbeiteten Grünmasse, um so größer ist in der Relation dazu der Abbau von dauerhaftem Bodenhumus. Diese umgekehrte Proportionalität ist besonders dann gegeben, wenn das eingebrachte Pflanzenmaterial schnell mineralisiert werden kann und selbst wenig zur Humusbildung beiträgt.

Gründüngungsmaßnahmen haben im allgemeinen auch deutlich positive Auswirkungen auf die betriebswirtschaftliche Bilanz. Bei diesen Betrachtungen sollte auch die ökologische Ökonomie nicht außer acht gelassen werden. Nach MÜLLER-SÄMANN (1986) geht z.B. einem Betrieb bei einem Bodenabtrag von $2,5 \mathrm{~cm}$ im Jahr ca. $2 \%$ des Humusgehalts und damit etwa $500 \mathrm{~kg}$ Stickstoff/ha durch Erosion verloren. Wird dieser Abtrag durch Bodendeckung mit Gründüngungspflanzen in risikoreichen Perioden gemindert, dann erfährt der Betrieb eine nicht unbeträchtliche Verlustminimierung.

Der standortgerechte Landbau strebt durch die Einbeziehung mulchliefernder Bäume und Sträucher in die Anbaufläche (Agroforstwirtschaft), durch Mischkultur, Gründüngung und durch ein Wirtschaften in möglichst geschlossenen Biomassekreisläufen (Kompostwirtschaft...) eine Erhaltung und Verbesserung der Bodenfruchtbarkeit an (ERGER \& KORUS, 1995). 
Der Schutz der Böden vor Erosion und Degradation erfolgt dabei nicht durch aufwendige und ertragsmindernde Zusatzmaßnahmen, sondern kostenarm und effizient durch biologische Verfahren: Eine möglichst ganzjährig hohe Bodenbedeckung, die Erhaltung und Vermehrung der organischen Substanz und eine verbesserte Bodenstruktur verhindern eine übermäßige Erwärmung und Austrocknung des Bodens, die Ablösung von Bodenpartikeln und die Zerstörung der Bodenstruktur durch den Aufprall von Regentropfen auf den ungeschützten Boden, erhöhen die Infiltrationskapazität der Böden und bremsen den Oberflächenabfluss. Die möglichst geschlossene, ganzjährige Vegetationsbedeckung des Bodens stellt dabei den wirksamsten Schutz vor Bodendegradation und -Erosion dar. Ein hoher Bodenbedeckungsgrad kann durch ein möglichst dichte Aussaat der Kulturpflanzen und Gründüngung als Heckenreihen, durch „mehrstöckigen“ Anbau ein- oder mehrsaisonaler Arten in Mischkultur erreicht werden.

Die beschrieben Nachteile lassen sich durch die Anwendung von Mulch vermeiden. Mulchen, d. h das Bedecken des Bodens mit totem Pflanzenmaterial, ist eines der effektivsten Bodenschutzverfahren. Eine Mulchbedeckung schützt den Boden vor Splasherosion und vor Austrocknung, dient den Bodenorganismen als Nahrung, trägt so zu einer Erhöhung der Makroporengehalte des Bodens bei und bremst eventuell auftretenden Oberflächenabfluss. Die mit dem Mulchauftrag verbundene Erhöhung des Gehaltes an organischer Substanz verringert über eine Verbesserung der Bodenfruchtbarkeit die Erodiertbarkeit der Böden. Insbesondere unter den sozioökonomischen Bedingungen eines Entwicklungslandes wie Vietnam sollten biologische Bodenschutzmaßnahmen, die auf eine Förderung des Pflanzenwachstums und eine Erhöhung der Bodenbedeckung abzielen, eine weit größere Bedeutung beigemessen werden, als rein mechanischen Erosionsschutzverfahren.

Abschießend ist festzustellen, dass ökologisch bewirtschaftete, in ihrer Erosionsschutzwirkung optimierte Agrofortsysteme durch eine erhebliche Verminderung erosionsbedingter Nährstoffverluste, durch das Recycling ausgewaschener Nährstoffe über den Blattfall der Bäume und die Verwendung von Heckenschnittmaterial als Mulch zu einer deutlichen Verlangsamung der Bodendegradation beitragen können.

Der Einsatz von Mischkulturen zusammen mit Hecken, Bäumen und Gründüngungspflanzen stellt jedoch eine erhebliche Erhöhung des Arbeitsaufwandes und der Betriebsorganisation gegenüber einer mit Monokulturen ausgestatteten Landschaft dar. 


\section{MODELLVORSCHLAG ZUR GESTALTUNG NACHHALTIGER LANDNUTZUNG}

„Nachhaltige Entwicklung bedeutet, dass die heutige Generation ihren Enkeln und Urenkeln die Möglichkeit offen hält, ihre eigenen Bedürfnisse zu befriedigen und einen eigenen Lebensstil zu wählen. Das geht nur, wenn mit endlichen Ressourcen Haus gehalten wird. Dahinter steht die Erkenntnis, dass Entwicklung und Armutsbekämpfung langfristig nicht auf Kosten der Lebensgrundlagen und Ökosysteme denkbar sind. Wirtschaftliches Wachstum, soziale Entwicklung und der Schutz der Umwelt müssen miteinander in Einklang stehen, um die Lebensbedingungen der Menschen zu verbessern“ (BMZ, 2002).

Das im Jahr 2025 verfügbare Ackerland wird im Vergleich zu 1990 spürbar zurückgehen: Nach Schätzungen in Afrika um zwei Drittel, in Asien um etwa ein Drittel und in Lateinamerika um etwa ein Fünftel. Das hat dramatische Folgen für die Sicherung der Ernährung. Wer die Bodenfruchtbarkeit erhält und die Desertifikation bekämpft, d. h. gefährdete Böden schützt und betroffene Böden rehabilitiert, mindert in den jeweiligen Ländern auch die Armut. Dies gilt auch im Umkehrschluss: Wer die Armut bekämpft, verringert damit auch die Bodendegradation. Die Nahrungsmittelversorgung wird gesichert, Hungersnöte beseitigt, Ursachen für Landflucht, Migration über Grenzen hinweg und Konfliktursachen werden behoben und letztlich ein Beitrag zu einer nachhaltigen Entwicklung geleistet. (BMZ-inforthek-spezial 054)

Die steigende Bevölkerungszahl wird dieses Probleme in nächster Zeit noch verstärken. Wenn die Erträge in den betroffenen Gemeinden nicht gesteigert werden, wird die Abwanderung in die Stadt und in die landwirtschaftlichen Gebiete im Tiefland und im Bergregenwald zunehmen. Es ist deshalb sinnvoll, mit Hilfe der vorhandenen Forschungsergebnisse nach Möglichkeiten der Ertragssteigerung in die Subsistenzwirtschaft zu suchen.

„Für die Gestaltung nachhaltiger Nutzungssysteme müssen daher die Grundlagen von Bodenqualität/ Bodenfruchtbarkeit für die einzelnen Nutzungssysteme bekannt sein. Die Nachhaltigkeit landwirtschaftlicher Nutzungssysteme wie auch die Regenerationsfähigkeit tropischer Wälder darf in der externen Nährstoffbilanz langfristig nicht negativ sein, wobei je nach Bodennährstoffvorrat das Defizit über eine gewisse Zeitdauer ausgeglichen werden kann“(GEROLD, 2002). 
Darauf aufbauend wurden in dieser Arbeit für das Untersuchungsgebiet Dialinh, (Babe) im Bergland Nordvietnams die sozioökonomischen Bedingungen im Zusammenhang mit der landwirtschaftlichen Nutzung und wichtige die Bodenfruchtbarkeit bestimmende Parameter analysiert.

Ableitend aus diesen Ergebnissen soll nachfolgend ein Modellvorschlag erarbeitet werden, unter den Gesichtspunkten, die Erosion zu stoppen, fruchtbare Böden zu erhalten und damit den Lebensstandard der ansässigen Bevölkerung zu steigern.

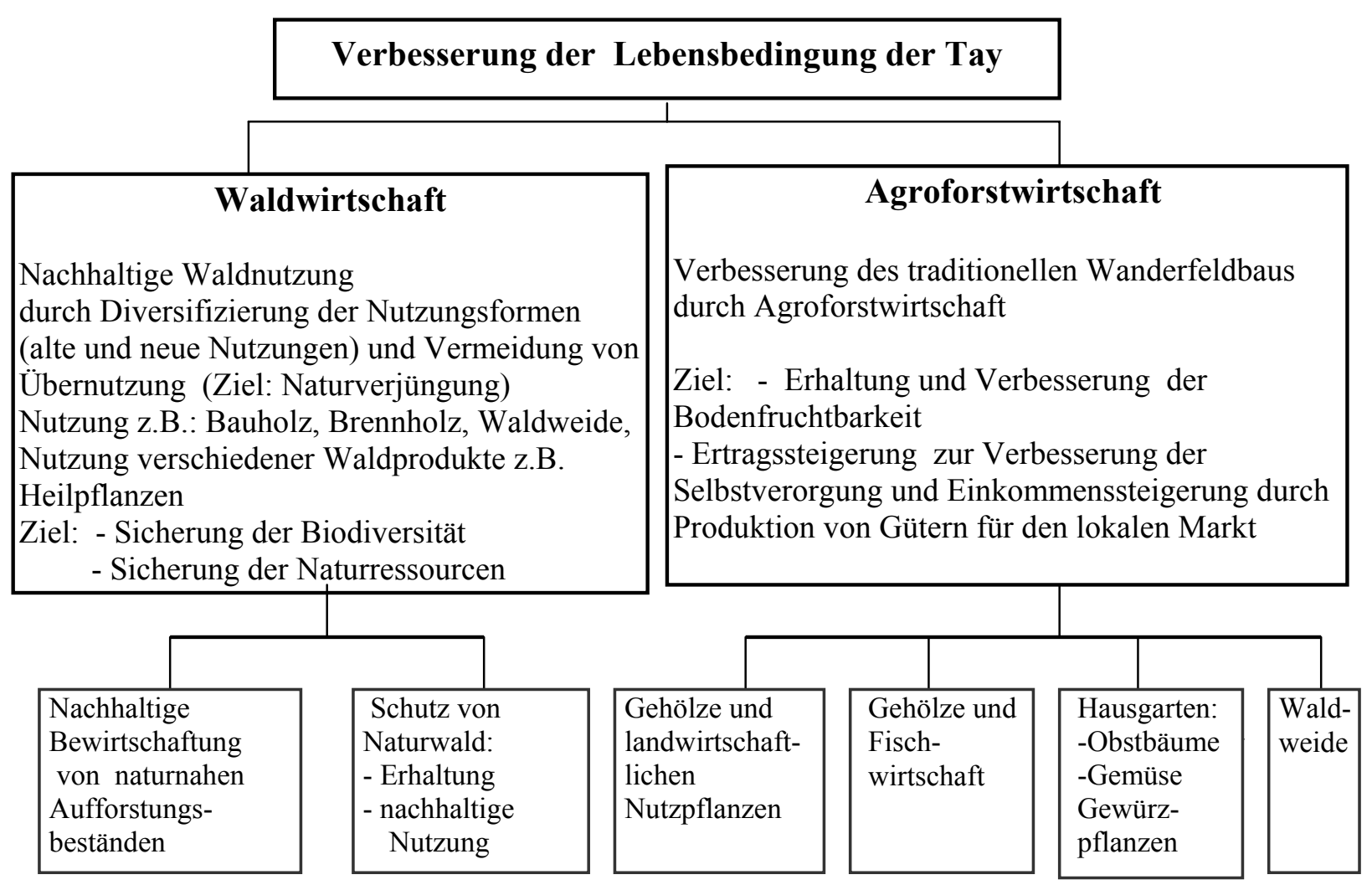

Abb.52: Modellvorschlag zur Gestaltung einer nachhaltiger Landnutzung und

\section{Waldwirtschaft}

Das Oberziel für die künftige Gestaltung der Landnutzung in diesem Gebiet ist die Verbesserung der Lebensbedingungen der ansässigen Tay-Familien. Grundlage dafür bildet der Schutz ihrer natürlichen Umwelt, d.h. Schutz der noch vorhandenen Naturwälder und Erhaltung der Bodenfruchtbarkeit. Unterziele sind deshalb die Produktion von lebensnotwendigen Gütern in Landnutzungssystemen, die die Bodenfruchtbarkeit nachhaltig sichern und verbessern, sowie nachhaltige Waldwirtschaft. Da sich diese Arbeit im wesentlichen mit der Verbesserung der landwirtschaftlichen Nutzung befasst, wird nachhaltige Waldnutzung auch nur kurz abgehandelt. 
Traditionell bilden Waldwirtschaft und Brandrodungsfeldbau die wichtigsten Komponenten der Landnutzung. Einen schematischen Überblick über Vorschläge zu ihrer Verbesserung vermittelt

Der Schutz des Naturwaldes durch die ansässige Bevölkerung könnte im Konkreten durch folgende Maßnahmen unterstützt werden:

- Vergütung, die der gesellschaftlichen Wertzumessung für den Wald entspricht

- Regelungen für saisonale produkt- und mengengebundene Nutzungen, die die nachhaltige Produktion gewährleisten

- Waldweide: Regelungen für die zeitweilige Nutzung mit dem Schwerpunkt in der Trockenzeit bei Futterknappheit

- Bewirtschaftung von Aufforstungsbeständen: gemeinschaftliche Baumschule, familiengebundene Pflanzung und Management der Gehölze

- Aufforstung mit Waldbaumarten, die Nutzung für Bevölkerung ermöglichen und eventuell damit zusätzliches Einkommen ermöglichen

In Tab.36 ist das Modell zur Waldnutzung kurz zusammengefasst.

Tab. 36: Vorschlag zum Managment von Wald in Tay - Dörfern

\begin{tabular}{|c|c|c|c|c|c|c|}
\hline Modell & Gehölzart & $\begin{array}{l}\text { Eigen- } \\
\text { bedarf }\end{array}$ & $\begin{array}{l}\text { Ver- } \\
\text { kauf }\end{array}$ & $\begin{array}{l}\text { Ver- } \\
\text { wendung }\end{array}$ & Ausbildung & $\begin{array}{l}\text { Förder- } \\
\text { maßnahme }\end{array}$ \\
\hline $\begin{array}{l}\text { Waldbewirtschaftung } \\
\text { 1.Schutz von } \\
\text { Naturwald Entlohnung } \\
\text { - Erhaltung } \\
\text { - nachhaltige Nutzung }\end{array}$ & & $\mathrm{X}$ & & $\begin{array}{l}\text { ES } \\
\text { ES }\end{array}$ & & Entlohnung \\
\hline $\begin{array}{l}\text { 2. Bewirtschaftung } \\
\text { von } \\
\text { Aufforstungsbeständen }\end{array}$ & $\begin{array}{l}\text { Tectona- } \\
\text { grandis } \\
\text { Bambusa } \\
\text { asundinace } \\
\text { ae } \\
\text { Leucaena } \\
\text { glauca }\end{array}$ & $\begin{array}{l}\mathrm{x} \\
\mathrm{xx}\end{array}$ & $\begin{array}{l}\mathrm{xx} \\
\mathrm{x}\end{array}$ & $\begin{array}{l}\text { Ba, ES } \\
\text { ES } \\
\text { Fu; BV, } \\
\text { ES }\end{array}$ & $\begin{array}{l}\text { Saatgut- } \\
\text { beschaffung } \\
\text { und -behandlung } \\
\text { Pflanzenanzucht, } \\
\text { Pflanztechnik } \\
\text { Schutz und Pflege }\end{array}$ & $\begin{array}{l}\text { Entlohnung } \\
\text { Saatgut }\end{array}$ \\
\hline
\end{tabular}

$\mathrm{Fr}=$ Frucht

$\mathrm{Fu}=$ Futter
$\mathrm{Bh}=$ Brennholz

$\mathrm{Ba}=$ Bauholz
$\mathrm{ES}=$ Erosionsschutz

$\mathrm{BV}=$ Bodenverbesserung $\mathrm{x}=$ zutreffend

$\mathrm{xx}=$ überwiegend 


\section{Landwirtschaft}

Zur Verbesserung der landwirtschaftlichen Nutzung durch Erhaltung der Bodenfruchtbarkeit wurde in dieser Arbeit das alternative Agroforstsystem mit Mulch im Vergleich zum traditionellem Wanderfeldbau untersucht. Die Vorteile des Agroforstsytems mit Mulch beruhen darauf, dass es durch seine Mischung verschiedener Feld- und Baumkulturen ein an der Natur orientiertes System darstellt.

Wesentliche Vorteile des Agroforstsystems hinsichtlich Bodenerhaltung und Bodenfruchtbarkeit gegenüber traditioneller Nutzung wurden bereits in Kap. 6.3 erarbeitet.

Im Bergland von Vietnam wurden bereits seit 1996 praktische Erfahrungen mit dem Agroforstsystem mit Mulch seitens der Thai Nguyen Universität gesammelt. Diese Erfahrungen und die Untersuchungsergebnisse der vorliegenden Arbeit wurden in dem folgenden Modellvorschlag verarbeitet.

Ein anstrebenswertes Ziel wäre es, den Wanderfeldbau schrittweise durch das Agroforstsystem mit Mulch $\mathrm{zu}$ ersetzen. Aufgrund der Eingliederung von Erosionsschutzmaßnahmen (Terrassenanbau, Heckenreihen, Mulchen) ist das Agroforstsystem bis $30 \%$ Hangneigung geeignet. In der Abb.53 ist ein Vorschlag unterbreitet bezüglich der Verteilung von Land- und Forstwirtschaft (oder Baum- und Feldkulturen) in Abhängigkeit von der Hangneigung, um ausreichend Erosionsschutz zu erhalten.

Hangneigung $>30^{\circ}$

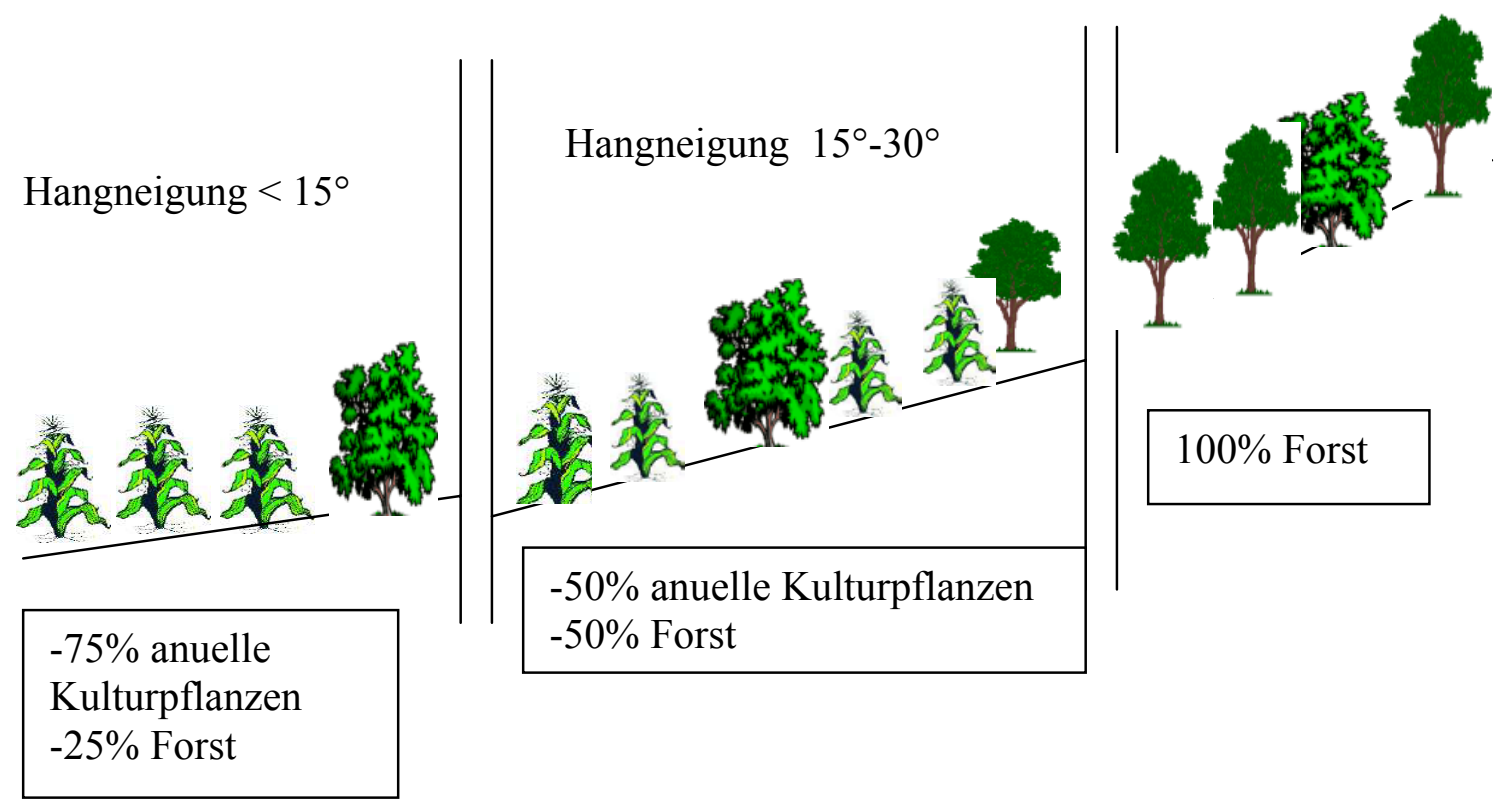

Abb. 53: Verteilung von Land-und Forstwirtschaft in Abhägigkeit von der Hangneigung 
Bei Hangneigungen $>30^{\circ}$ muß man bei landwirtschaftlicher Nutzung trotz Erosionschutzmaßnahmen mit Bodenabtrag rechnen. Eine Verschlechterung der Bodenqualität ist dann unabdingbar. Forstwirtschaft sollte daher als einzige Nutzung angestrebt werden.

Da das Agroforstsystem Maßnahmen zur Erhaltung der Bodenfruchtbarkeit beinhaltet, sind Brachezeiten nicht mehr notwendig. Eine permanente Nutzung der Flächen wird somit möglich. Aufgelassene Brandrodungsfelder vor allem in steileren Hanglagen und degradierten Standorten könnten aufgeforstet werden. Später ist eine neue Nutzung dieser Sekundärwälder möglich (s. Waldwirtschaft).

Da das Agroforstsystem Maßnahmen zur Erhaltung und Verbesserung der Bodenfruchtbarkeit beinhaltet, wird dadurch eine Rehabilitierung bereits degradierter Standorte möglich. Dies wird am Beispiel der in dieser Arbeit untersuchten Agroforstparzelle mit Erfolg praktiziert. Nach 3 jähriger Nutzung einer ehemaligen Alang - Alang - Brache in Agroforstwirtschaft ist bereits eine deutliche Verbesserung der Bodenfruchtbarkeit gegenüber dem traditionellen Wanderfeldbau sichtbar.

Bei dem vorgeschlagenem Agroforstsystemsystem handelt es sich um eine Mischung von Feld- und Baumkulturen, wobei Leguminosenhecken zum Erosionsschutz in Konturstreifen gepflanzt mit Bergkulturen wechseln (Abb. 54). Zwischen den Feldkulturen sind Obstbaumkulturen gepflanzt.

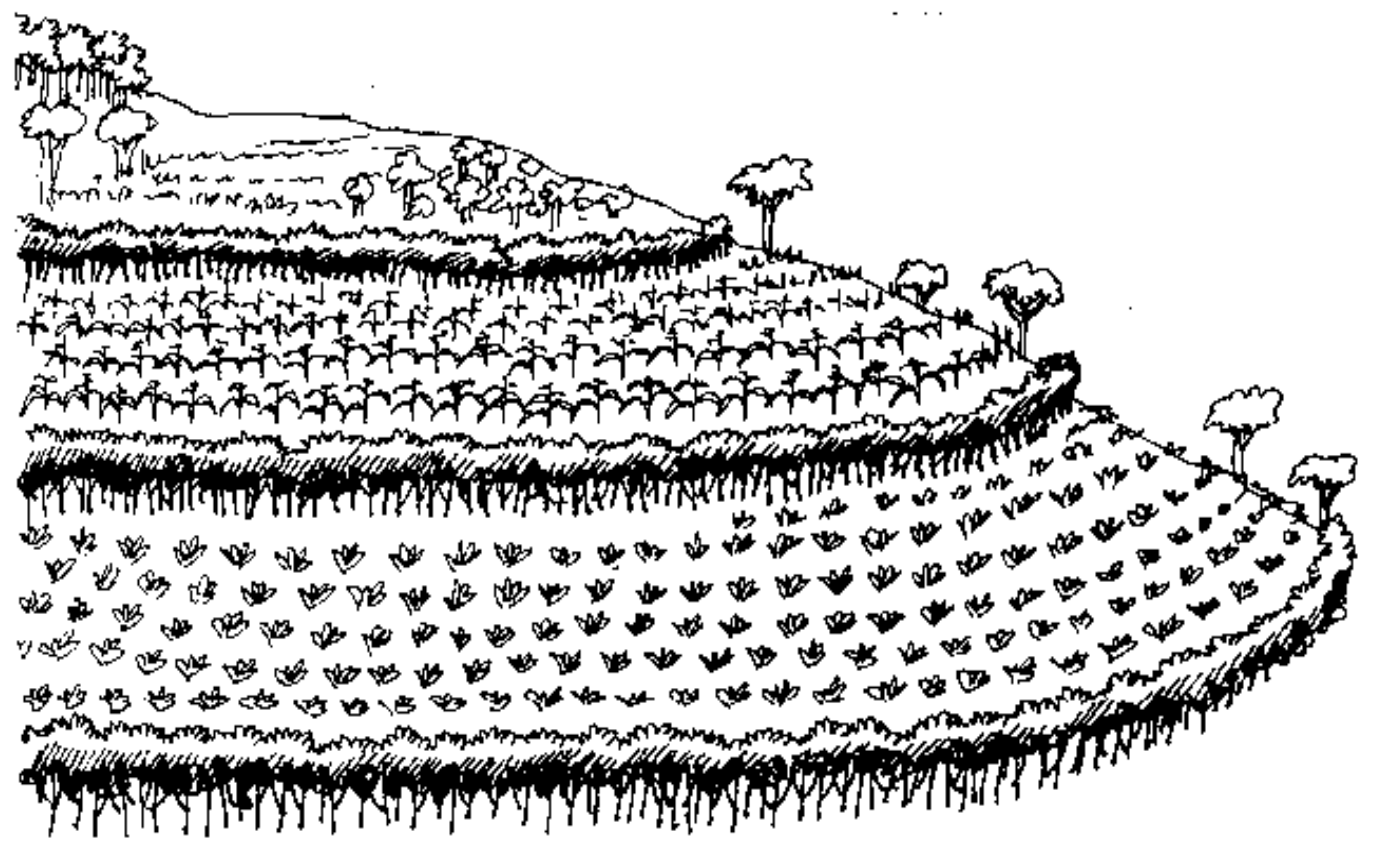

Abb.54: Konturstreifenanbau 
Weitere Details zur praktischen Umsetzung sind in Kapitel 8 insbesondere unter dem Gesichtspunkt des Erorsionsschutzes dargestellt.

In Tab. 37 ist ein Vorschlag zum Management von Agroforst in Tay - Dörfern unterbreitet.

Tab. 37: Vorschlag zum Management von Agroforst in Tay-Dörfern

\begin{tabular}{|c|c|c|c|c|c|c|}
\hline Modell & Gehölzart & $\begin{array}{l}\text { Eigen- } \\
\text { bedarf }\end{array}$ & $\begin{array}{l}\text { Ver- } \\
\text { kauf }\end{array}$ & $\begin{array}{l}\text { Ver- } \\
\text { wendung }\end{array}$ & Ausbildung & $\begin{array}{l}\text { Förder- } \\
\text { maßnahme }\end{array}$ \\
\hline $\begin{array}{l}\text { Agroforstwirtschaft } \\
\text { 1.Hausgarten } \\
\text { Obstbäume und } \\
\text { Nutzpflanzen } \\
\text { * Baumschule } \\
\text { * Pflanzung } \\
\text { * Pflege } \\
\text { * Ernte }\end{array}$ & $\begin{array}{l}\text { Euphoria } \\
\text { longana } \\
\text { Citrus spp, } \\
\text { Ziziphus } \\
\text { Jujuba, Jack } \\
\text { fruit, Litchee, } \\
\text { mango ... }\end{array}$ & $\begin{array}{l}\mathrm{xx} \\
\mathrm{xx} \\
\mathrm{xx} \\
\mathrm{xx} \\
\mathrm{xx} \\
\mathrm{xx}\end{array}$ & $\begin{array}{l}\mathrm{x} \\
\mathrm{x} \\
\mathrm{x} \\
\mathrm{x} \\
\mathrm{x} \\
\mathrm{x}\end{array}$ & $\begin{array}{l}\text { ES, Fr } \\
\text { ES, Fr } \\
\text { ES, Fr } \\
\text { ES, Fr } \\
\text { ES, Fr } \\
\text { ES, Fr }\end{array}$ & $\begin{array}{l}\text { Saatgutbe- } \\
\text { schaffung und } \\
\text { - behandlung } \\
\text { Pflanzen- } \\
\text { anzucht, } \\
\text { Pflanztechnik } \\
\text { Schutz und } \\
\text { Pflege }\end{array}$ & $\begin{array}{l}\text { Entlohnung } \\
\text { Saatgut }\end{array}$ \\
\hline $\begin{array}{l}\text { 2. Gehölze und } \\
\text { landwirtschaftlich } \\
\text { Nutzpflanzen }\end{array}$ & $\begin{array}{l}\text { Leguminosae } \\
\text { Tephrosia } \\
\text { candida } \\
\text { Bohnenarten }\end{array}$ & $\begin{array}{l}\mathrm{xx} \\
\mathrm{x} \\
\mathrm{xx}\end{array}$ & $\mathrm{X}$ & $\begin{array}{l}\text { Fu, BV,ES } \\
\mathrm{Fu}, \mathrm{ES} \\
\mathrm{Fu}, \mathrm{BV}\end{array}$ & & \\
\hline 3. Waldweide & Futterpflanzen & $\mathrm{x}$ & & $\mathrm{Fu}, \mathrm{ES}$ & & \\
\hline
\end{tabular}

$\mathrm{Fr}=$ Frucht $\quad \mathrm{Bh}=$ Brennholz $\quad \mathrm{ES}=$ Erosionsschutz

$\mathrm{Fu}=$ Futter $\quad \mathrm{Ba}=$ Bauholz $\quad \mathrm{BV}=$ Bodenverbesserung $\mathrm{x}=$ zutreffend $\mathrm{xx}=$ überwiegend

Neben traditionellen Feldkulturen des Wanderfeldbaus, die zur Ernährung der Familien bestimmt sind (Maniok, Mais, Sojabohne, Trockenreis), beinhaltet das Agroforstsystem auch den Anbau neuer Kulturen: Hecken (Tephrosia cadinda, Leucacia glauca,) Calliandra, Gliricilia sepium (madre de cacao), die neben Erosionschutz und Erhaltung der Bodenfruchtbarkeit auch der Ernährung der Tiere in der Trockenzeit bzw. als Brennholz dienen können. Über die Eingliederung von Obstbaumkulturen, wie sie bereits in Hausgärten vorzufinden sind, ist eine weitere Diversifizierung der Nutzung gegeben. Neue Kulturen bzw. Diversifizierung des Anbaus schaffen nicht nur neue Nutzungsmöglichkeiten, sondern können auch die Ernährungssituation der Bauern verbessern und die Möglichkeit für ein zusätzliches Einkommen durch Verkauf von Produkten (z.B. Obst) auf dem lokalen Markt ermöglichen. 
Maßnahmen zur Ertragssteigerung könnten neue Sorten bzw. Pflanzenschutz beinhalten, sind jedoch stark von der finanziellen Situation der Familie oder finanzieller Unterstützung von außen abhängig.

Seit das Agroforstsystem seit 4 Jahren von der Thai Nguyen Universität praktisch umgesetzt wurde, haben es bisher nur einige wenige Bauern mit höherer Ausbildung angenommen.

Die Mehrzahl der Bauern haben noch keine Erfahrung mit dem in der Untersuchung vorgestelltem System. Eine Beratung und Hilfestellung der Bauern, die meistens keine Ausbildung haben, ist daher unabdingbar. Die Einrichtung von landwirtschaftlichen Beratungsstellen bzw. die landwirtschaftliche Unterstützung vor Ort durch die Universität oder staatliche Berater ist notwendig.

Nach entsprechender Beratung und Unterstützung bei der Umsetzung ist eine zukünftig gute Akzeptanz des Agroforstsystems denkbar. Bei der Umsetzung und Bewirtschaftung des Agorforstsystems könnte man die bisherigen Erfahrungen der Bauern aus der Nutzung von Hausgärten (agroforstwirtschaftliches System in kleinem Rahmen) und in der Bewirtschaftung von Terrassen nutzen (neues System ähnlich vorhandener Systeme). Das Agroforstsystem ist nur am Anfang arbeitsaufwendiger als die traditionelle Brandrodungswirtschaft, zudem ist die verwendete Technik einfach und die Kosten sind relativ gering. Da die Mehrzahl der Bauern aber nur in Subsistenzwirtschaft leben ist eine finanzielle Unterstützung von außerhalb zumindest für den Anfang absolut notwendig. Angepasste Bodennutzungskonzepte sind notwendig, um den weiteren Rückgang des Waldes zu verringen. Konzepte, die bei möglichst gleichzeitiger Intensivierung des Anbaus eine langfristige Stabilität des Nährstoffhaushaltes auf den Nutzungsparzellen beinhalten, sind anzustreben (GEROLD, 1997). 


\section{ASPEKTE DER PRAKTISCHEN UMSETZUNG VON EROSIONSSCHUTZMAßNAHMEN}

Selbst ein gut vor Bodenerosion geschütztes, ökologisch angepasstes Anbausystem ist jedoch per se nicht in der Lage, die Fruchtbarkeit bereits stark degradierter Standorte zu regenerieren. Dem vorbeugenden Schutz der Böden vor Degradation kommt somit eine entscheidende Bedeutung zu.

\subsection{Terrassierung}

Das Vermessen und Markieren von Linien gleicher Höhe (Isohypsen) an einem Hang ist Voraussetzung für die Realisierung verschiedener Erosionschutzmaßnahmen, wie z.B des Konturanbaus sowie von Terrassen und dem Errichten von Abflusshindernissen.

Der vorliegenden Literatur zufolge ist der A-Rahmen offensichlich das in Vietnam und sowie Philippinen meist verwendete Gerät (DANG, 1998; THAI, 1996; TACIO, 1988, 1991). Der A-Rahmen ermöglicht sowohl die Festlegung von Konturlinien, als auch die Bestimmung eines geringen Gefälles, wie es z.B. bei der Anlage von Ableitungsgräben und -terrassen notwendig ist.

Zum Bau des Gerätes genügen drei 1,5 bis 2,0 m lange Holzlatten bzw. annähernd gerade Äste, die $\mathrm{zu}$ einem Gestell, welches die Form des Buchstabens „A“ hat, zusammengebunden bzw.- genagelt oder- geschraubt werden. Hinzu kommt ein Faden, welcher, am unteren Ende mit einem Lot (z.B.: Stein, Flasche) beschwert, von der Spitze des aufgestellten Rahmens herunterhängt, wobei er die Querlatte durchkreuzt (Abb. 55)

Zur Eichung wird das Gerät mit je einem Bein auf jeweils einen markierten Festpunkt gestellt. Nachdem das Lot zur Ruhe gekommen ist, kennzeichnet man die Querlatte dort, wo sie von dem Faden geschnitten wird. Danach wird der Rahmen, um $180^{\circ}$ gedreht, erneut auf die Festpunkte plaziert. Der neue Schnittpunkt wird ebenfalls markiert. Die Mitte zwischen beiden Schnittpunkten ist der O-Punkt. Kreuzt ihn das Lotband, so stehen beiden Beine des A- Rahmens auf Positionen gleicher geodätischer Höhe. Falls nicht ausgeschlossen werden kann, dass sich das Holz verzieht, sollte die Eichung zu Beginn eines Arbeitstages überprüft und gegebenenfalls erneut vorgenommen werden 


\section{Abb.55: A-Rahmen: Einfache Geräte zur Vermessung von Höhenlinien}

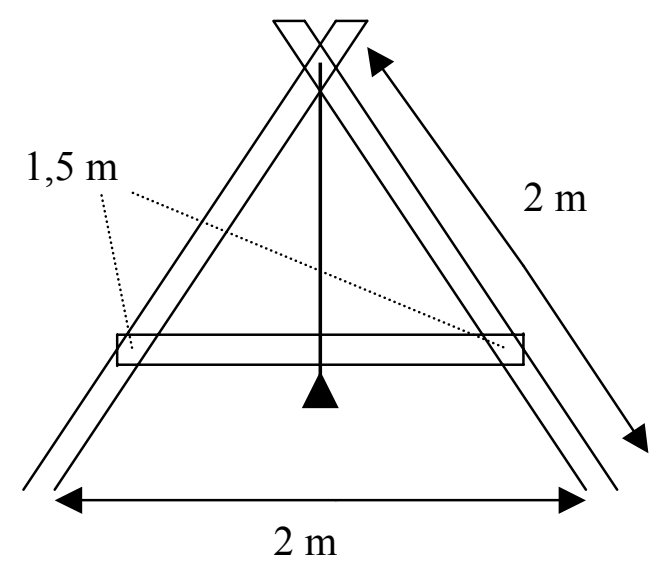

Beim Vermessen einer Höhenlinie steht immer ein Bein auf einem bereits festgelegten Punkt, während mit dem freien Bein durch hangaufwärts bzw. hangabwärts gerichtetes Tasten der nächste Punkt gesucht wird.

\section{2 weitere Erosionsschutzmaßnahmen}

Als Elemente einer Modernisierung und Intensivierung kommen nun in Frage: Erosionsschutzlinien, Wiedereinführung von Bäumen im Feldbereich unter Zuziehung neuer Baumarten, Forstbänder, erweiterte Mischkulturen. Diese Elemente müssen zu einem produktiv und nachhaltig funktionierenden System zusammengefügt werden, das einen möglichen Prototyp für weitergehende Feinentwicklungen darstellt. Der seit 1995 in der Thainguyen Provinz in Erprobung genommene, durch Vorversuche abgesicherte Vorschlag gliedert sich in zwei Teile (Abb. 56):

- die ( Agro-)forst-Komponente und

- die Rotation der eigentlichen Felder

Agroforstsystem

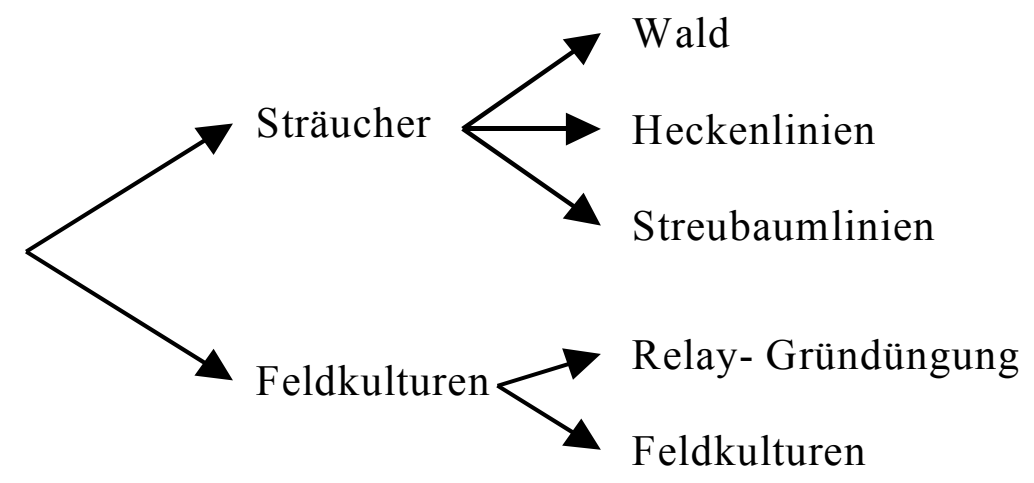

Abb. 56: Synthese: Ein agroforstliches Rotationsystem 
Es enthält als Elemente:

- Wald mit Nutz- und Brennholzarten (Teak, Leucaene, einige Akazienarten...) am ganzen Oberhang oder ab und zu von 6-10 m Breite, annähernd den Höhenlinien parallel geführt, sollen die langen Hänge unterbrechen - dazwischen liegende Felder mit 20-30 m Breite.

- Streubäume, früher regellos locker in allen Altersstufen über die Felder verteilt, sollen nun in einer den Höhenlinien folgenden Reihe, durch die Feldmitte, in Abständen von 5$10 \mathrm{~m}$ angepflanzt werden.

- Heckenlinien sollen oder können die Waldstreifen säumen, Schnittmaterial für Mulchzwecke (Alley croping) oder Tierfutter liefern. Geeignet sind Leucaena leucocephala, Flemingea conguesta und ganz besonders Tephrosia candida. Heckenlinien, wiederum entlang der Höhenlinien, können zusätzlich besonders breite Felder gliedern und dabei helfen, eine leichte Terassierung herbeizuführen.

Alle drei „Forst“ - Komponenten dienen wirkungsvoll der Wasserrückhaltung, dem Erosionsschutz und dem Humusaufbau (Heckenschnitt und Laubabwurf)

Die notwendige Abkehr von extensivem degradierendem Wanderfeldbau, ist prinzipiell möglich. Danach sollten Korrekturen, Anpassungen und Variationen durch Bauern und Landesforschungseinrichtungen erst recht beginnen. Ohne eine Chance $\mathrm{zu}$ eigenen Erfolgserlebnissen bliebe sonst ein solcher Wandel der Agrikultur fremd und aufgesetzt.

Die zwei Grundprinzipien des ökologischen Landnutzungssysteme sind folgende:

- Aufbau bzw. Erhalt der Bodenfruchtbarkeit nach dem Vorbild der Natur

- Gesunder Boden= Gesunde Kulturen = Gesundmenschen .

Bei diesem Verfahren werden die Hecken zu Beginn der Anbausaison auf 0,5- 1 Meter Höhe zurückgeschnitten. Laub und feines Astwerk werden als nährstoffreicher Mulch in die Gänge zwischen den Strauchreihen gelegt- Stärkere Äste werden als Brennholz genutzt. Die annuellen Kulturen werden in den Mulch gesät bzw. gepflanzt und während des Wachstum nochmals gemulcht, denn während des Vegetationszyklus der annuellen Kultur müssen die Sträucher 3-4 mal im Jahr zurückgeschnitten werden, um eine zu 
starke Beschattung zu vermeiden. Die Produktionsmenge der Heckenreihen beträgt 6-8 t/ha/a (PHIEN \& SIEM, 1998).

Bei der Auswahl von Gründüngung ist nach MüLlER-SÄMANN (1986) auf eine Reihe von Eigenschaften zu achten, um die Erfolgsaussichten der Gründüngungsmaßnahme zu erhöhen. (vgl.Tab.38). Ebenso beeinflussen selbstverständlich die natürlichen Standortfaktoren (Klima, Bodeneigensachten) die Auswahl.

Tab. 38: Wünschenswerte Eigenschaften von Gründüngungspflanzen (Quelle: MÜLLER-SÄMANN (1986)

\begin{tabular}{|l|}
\hline I. Genetische Eigenschaften: \\
\hline hohe Biomassenproduktion in relativ kurzer Zeit \\
hoher Anteil von grünen, nicht verholzten Pflanzenteilen \\
Fähigkeit zur schnellen Bodendeckung \\
Durchsetzungsvermögen gegenüber dominanten Unkräutern \\
Starkes, tiefgehendes Wurzelwerk \\
Hohes Nährstoffaufschlussvermögen \\
N-Fixierung (Leguminosen) \\
Hohe Affinität zu Mycorrhiza \\
\hline II. Phytosanitäre Eigenschaften: \\
\hline robust und unempfindlich \\
hohe Krankheits- und Schädlingsresistenz \\
keine Affinität zu Krankheitserregern und Schädlingen (Wirtspflanzenfunktion), die \\
Nutzpflanzen gefährden könnten (im Idealfall Feindpflanze) \\
\hline III. Ackerbauliche Eigenschaften: \\
\hline Anbau erfordert geringen Aufwand an Arbeit und speziellem“ know-how“ \\
Leicht Verfügung des Saatgutes am Standort \\
Kontrollierbarkeit der Gründüngungspflanze/geringe Verunkrautungsgefahr \\
Mögliche Nebennutzung der Pflanze (z.B. als Futter)
\end{tabular}

Bei der Aussaat von Gründüngungspflanzen sollte im allgemeinen eine möglichst hohe Bestandsdichte angestrebt werden, damit:

- der Bestand möglichst schnell den Boden deckt und

- das Auflaufen unerwünschter Breikräuter unterdrückt,

- die Durchwurzelung der oberen Bodenschichten erhöht,

- der hohe Populationsdruck die Biomassenproduktion verstärkt und

- bei Leguminosen die Netto- N- Fixierung gesteigert,

- die Verholzung verringert und

- das C/N-Verhältnis verengt wird.

RosA ET AL. (1980) versucht Reihenmischkultur -bzw. Mulchversuche in den Feuchttropen der Philippinen. Sie legten hangparallele Reihen (ebenfalls) mit $L$. leucocephala an, wobei 10, 15, 20 Pflanzen pro laufenden Meter gesät wurden 
(Reihenabstand 1,5 Meter, Gefälle 30\%). Leucaena wurde 90 Tage vor dem Mais gesät und nach 3 Woche ausgedünnt. Der Mais wurde im Abstand von $25 \mathrm{~cm}$ auf kleinen Hügeln zwischen den Hecken gesät (26.667 Pflanzen pro Hektar gegenüber 53.333 in der Kontrolle ohne Leucaena): Die Erträge pro Maispflanzen waren im Reihenanbau über 100\% höher. Über den Mehrertrag pro Einzelpflanzen konnte der Verlust an Fläche für den Mais (gegenüber traditioneller Pflanzenweise) mehr als wettgemacht werden. Die Erosion wurde gemindert, die Infiltration verbessert.

Diesen Fragen sollte in Zukunft größere Bedeutung zugemessen werden, um bei einer Einführung neuer Methoden in der Landbewirtschaftung eine größere Akzeptanz und eine bessere Umsetzung zu erreichen.

Wenn aber Bauern die Notwendigkeit, die Bodenfruchtbarkeit zu verbessern, einsehen und die Prinzipien des Recyclings, der Vielfalt, der Integration und ständigen Bodenbedeckung verstehen, können viele eigenständige Wege der Ertragssteigerung, der Erhaltung und Verbesserung von Bodenfruchtbarkeit gefunden werden, die nicht so arbeitsintensiv sein müssen. Je größer die Vielfalt der Anbaumethoden, je größer die Vielfalt der Aktivitäten und ihre Integration, desto wahrscheinlicher wird es, dass der Arbeitseinsatz besser über das gesamte Jahr verteilt wird. Einige traditionelle Anbaupraktiken mögen hier ihren festen Platz finden.

Die Entscheidung der Betriebsleiter, die Pflanzen Tephorocia candida und Leuceana glauca als Erosionsschutzmaßnahme zur Gewährleistung langfristiger Nutzung auf landwirtschaftlichen Flächen in Hanglagen einzusetzen, ist von verschiedenen Faktoren abhängig. Als mögliche Entscheidungskriterien werden die Arbeitskraftaustattung des Betriebes, die Betriebsgröße, der Umfang der beratungsdienstlichen Tätigkeit, die Bildung des Betriebsleiters, die Besitzstruktur der bewirtschafteten landwirtschaftlichen Fläche sowie die Kapitalausstattung des Betriebes herangezogen.

Der Hypothesenstern, der in Abb. 57 dargestellt ist, veranschaulicht die nach Bedarf weiter zu untersuchenden Entscheidungskriterien, die für die Einführung der Erosionsschutzmaßnahme von Bedeutung sind. Mit abnehmender Bedeutung einzelner Faktoren (sich verjüngende Spitze des Sterns) nimmt auch die Nutzungsintensität ab. 


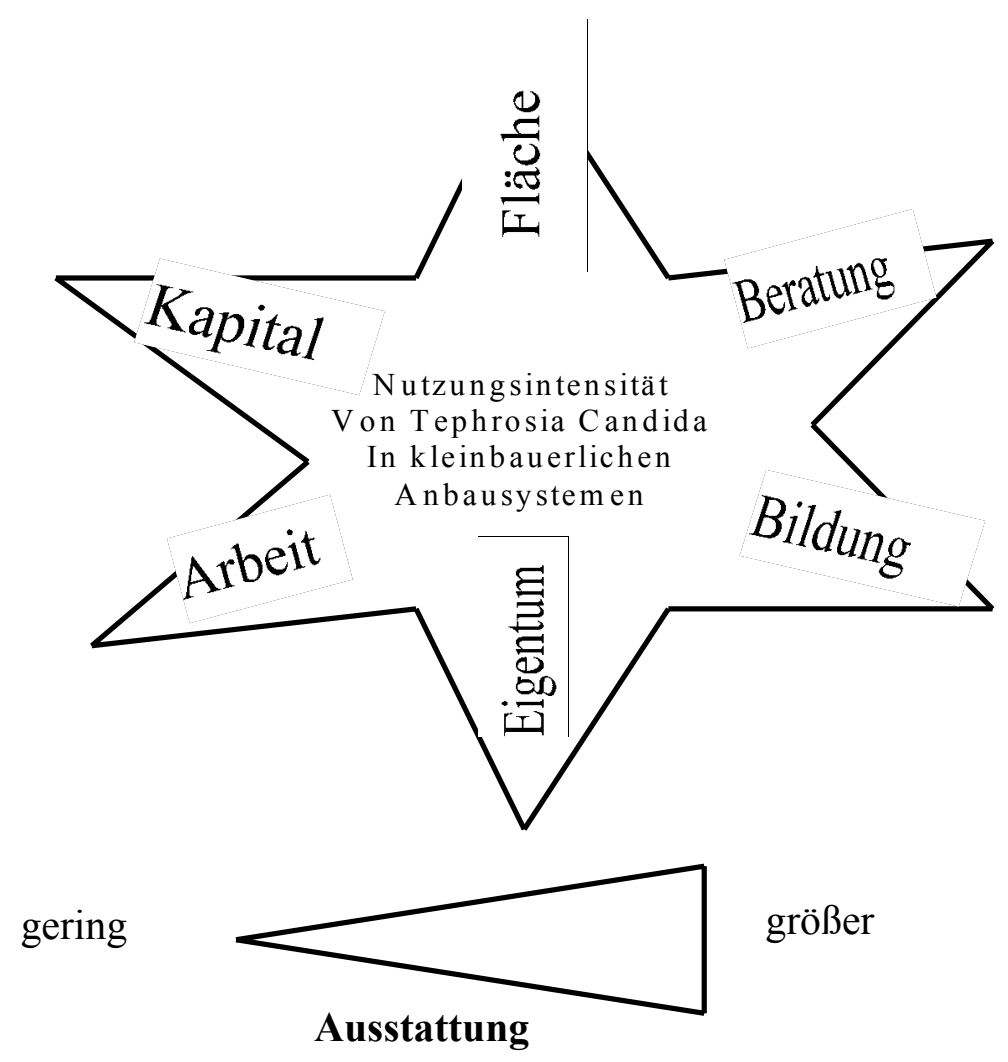

Abb. 57:Hypothesenstern

Um die Akzeptanz und den nachhaltigen Erfolg von Bodenkonservierungmaßnahmen zu gewährleisten, sind Programme und Maßnahmen seitens der Regierung, sowie die Unterstützung der Bauern vor Ort durch staatliche Agrarberater erfordert. Es ist notwendig, die angepasste Bodenkonservierung als einen Komplex miteinander vernetzter Ursachen und Wirkungen sowie entsprechender Maßnahmen zu betrachten. Im Mittelpunkt des integrierten Erosionsschutzes sollte immer die Anwendung standortgerechter Landbaumethoden stehen. So reduziert Agroforst mit Mulch nicht nur den Bodenabtrag sondern trägt allgemein zu einer nachhaltigen, risikoarmen, Erhaltung und Verbesserung der Bodenfruchtbarkeit und gesteigerten Agrarproduktion bei. 


\section{ZUSAMMENFASSUNG}

Die vorliegenden Untersuchungsergebnisse dokumentieren, dass Bodendegradierung durch Wassererosion ein zum Teil schwerwiegendes Problem in den Bergregionen Nordwestvietnams darstellt. Viele Böden verlieren ihre natürliche Bodenfruchtbarkeit durch Bodendegradierung. Langfristig gesehen kann so die Ernährungssicherung der Bevölkerung gefährdet sein. An den Standort angepasste Anbausysteme, die eine Erhaltung bzw. Verbesserung des langfristigen Ertragspotentials gewährleisten, müssen sofort umgesetzt werden, damit das Ernährungspotential in Zukunft sichergestellt ist.

In dieser Arbeit wurden zwei Landnutzungssysteme aus der Region Naduc - Dialinh, (Babe Distrikt) im Bergland NW-Vietnams miteinander verglichen: das Agroforstsystem mit Mulch, das neue landwirtschaftliche Techniken beinhaltet, und der konventionelle Feldanbau (traditioneller Wanderfeldbau mit Brandrodung). Agroforstsysteme mit Mulch scheinen gegenüber dem aktuellen traditionellen Wanderfeldbau mit Brandrodung einen deutlich niedrigeren Bodenabtrag und bessere Bodenfruchtbarkeit aufzuweisen.

In dieser Arbeit wurde untersucht, ob Bodenerosion als bedeutende Einflussgröße Bodendegradation und landwirtschaftlichen Ertrag in den o. g. Systemen beeinflusst. Zu diesem Zweck erfolgte eine ausführliche Analyse der agroökologischen Standortbedingungen für das Agroforstsystem mit Mulch und dem traditionellen Wanderfeldbau mit Brandrodung.

Der Schwerpunkt der vorliegenden Arbeit basiert auf:

1. soziökonomischen Erhebungen in Naduc-Dialinh

2. der detaillierten Darstellung der Bodenfruchtbarkeitsparameter in Abhängigkeit von der Nutzungsdauer.

3. der Darstellung der Auswirkungen der Wassererosion auf chemische und physikalische Bodeneigenschaften und deren Einfluss auf die Ertragsleistung der Böden.

Zu Punkt 2 und 3 wurden folgende Parameter analysiert (jeweils Ober- und Unterboden der beiden Systeme und zu Beginn und Ende der Regenzeit 2001):

- die Nährstoffkonzentrationen (Nt, $\mathrm{Ct}, \mathrm{P}_{2} \mathrm{O}_{5}$ nach Bray),

- austauschbare Kationen: $\mathrm{Ca}^{2+}, \mathrm{Mg}^{2+}, \mathrm{Na}, \mathrm{K}, \mathrm{Fe}^{2+}, \mathrm{Al}^{3+}, \mathrm{Mn}, \mathrm{pH}-$ Wert und Totalaufschluss von $\mathrm{Ca}^{2+}, \mathrm{Mg}^{2+}, \mathrm{Na}, \mathrm{K}, \mathrm{Fe}^{2+}, \mathrm{Al}^{3+}, \mathrm{Mn}, \mathrm{Zn}, \mathrm{Cu}, \mathrm{Pb}, \mathrm{S}, \mathrm{Cr}, \mathrm{Cd}, \mathrm{Co}$, Mo, $\mathrm{Ni}$

Die Erträge wurden ebenfalls erhoben. Zudem werden sowohl acker- und pflanzenbauliche als auch technische Maßnahmen beschrieben. 
Aus den sozioökonomischen Erhebungen wird zusammenfassend festgestellt:

Die typischen Nutzpflanzen der Tay-Bevölkerungsgruppe sind Monokulturen von Mais, Maniok, Nassreis oder Trockenreis. Die Erträge sind allerdings nicht stabil. Das Leben der Tay-Bevölkerung in Bergland trägt sich hauptsächlich durch die Nutzung natürlicher Ressourcen, besonders aus Boden, Wasser und Wald. Das Einkommen aus der Landwirtschaft beträgt durchschnittlich $80 \%$, während über 15\% des Einkommens aus der Waldproduktion generiert werden. Sowohl reich als auch arm klassifizierte Familien haben ein Einkommen von <1US\$/ Person/ Tag und liegen damit unter der Armutsgrenze nach FAO 2001. Die Bauern besitzen wenig oder keine Informationen über die Ursachen, Gefahren und Folgen der Bodendegradation. Die Übernutzung der natürlichen Ressourcen, bedingt u.a. durch die zu kurze Brachezeit, hat seine Ursachen in einem übermäßigen Bevölkerungswachstum. Dieses führt zu einem Nahrungsmittelmangel und folglich zur Waldrodung für den Anbau von Kulturpflanzen. Das ökologische Gleichgewicht wird dadurch gestört.

Bodenuntersuchungen und Geländeaufnahmen zeigen, dass $\mathrm{pH}-\mathrm{Wert}$ und Basensättigung im Untersuchungsgebiet sehr deutlich nach langjähriger landwirtschaftlicher Nutzung der Böden abnehmen (pH-Wert von 6,5 auf 4,4; V-Wert von 99,6\% auf 28,9\%). Besonders deutlich wird die Degradation durch landwirtschaftliche Nutzung sichtbar anhand der Veränderung der organischen Substanz, des Gesamtstickstoffgehaltes und der Kationen Austauschkapazität. Der Rückgang der organischen Substanz mit Dauer des Anbaus bewirkt einen Verlust an Austauschkapazität sowie Verminderung der mineralisierten Nährelemente. Die P- Verfügbarkeit nach Bray ist im Ober- und Unterboden als sehr gering einzustufen und die Abnahme der P- Verfügbarkeit ist sehr stark. Der Grund liegt darin, dass zum einen ein Teil der P-Verfügbarkeit durch Bodenerosion verloren geht und zum anderen aufgrund von Eisen- und Aluminiumanreicherung bei niedrigen $\mathrm{pH}-$ Werten eine verringerte Phosphorverfügbarkeit durch die irreversible Phosphatfixierung gegeben ist.

Bodenerosion findet im Bergland von Vietnam auf landwirtschaftlich genutzten Hängen vor allem in Folge hoher und konzentrierter Niederschläge während der Regenzeit von April bis September statt. Die Bodenerosion beträgt 43 -58,3 t/ha/a im 1.Jahr und 4.Jahr nach Brandrodung. Dagegen liegt sie unter Agroforst mit Mulch bei nur 3,7 t/ha/a. Bodenerosion ist die Hauptursache von Bodendegradation. Als Nährstoffverluste wurden 996-1234 Kg org. Substanz/ha/a, 73-82Kg N/ha/a, 854-1468 Kg K, 73-82Kg P/ha/a, $642-1035 \mathrm{Kg} \mathrm{Mg} / \mathrm{ha} / \mathrm{a}, 89-97 \mathrm{Kg} \mathrm{Ca} / \mathrm{ha} / \mathrm{a}$ gemessen und sind als schwerwiegend 
einzustufen. Der Kostenaufwand an NPK zum Ausgleich von Nährstoffverlusten durch Bodenerosion beträgt im Brandrodungsfeldbau 5.383.000-8.849.400 VND/ha/Jahr und entspricht damit dem 3-4 fachen des Jahreseinkommens der Tay-Familien. Dagegen liegt der Kostenaufwand im Agroforst mit Mulch bei nur 370.300/ha/ a.

Aufgrund hoher Abtragsraten und geringer Mineralreserven der Böden im Babe - Distrikt kommt es am Untersuchungsstandort zu einer irreversiblen Bodendegradation durch Bodenerosion mit erheblichem Rückgang der Bodenfruchtbarkeit. Der Maisertrag auf der Parzelle im 4.Jahr nach Brandrodung betrug nur ein Drittel des 1. Jahr. Eine Grundlage für die Erhaltung der Böden, können biologische Maßnahmen darstellen, welche zu einer nachhaltigen Verbesserung der Bodenfruchtbarkeit und einer besseren biologischen Aktivität des Bodens führen. Dazu werden u.a. Bodennutzungssysteme wie agroforstliche Systeme und Mischanbau mit Mulch, welche natürliche Pflanzengesellschaften zum Vorbild haben, ausführlicher diskutiert. Bei den in optimal geschützten, ökologisch bewirtschafteten Agroforstsystemen gemessenen Abtragsraten (3,69 t/ha/a) bleiben die Verluste an Nährstoffen und organischer Substanz auf ein tolerierbares Ausmaß begrenzt. Die nach wie vor in solchen gut gegen Bodenerosion geschützten Agroforstsystemem auftretenden Nährstoffverluste können durch den Streufall von den Bäumen und die Verwendung von Heckenschnitt als Mulch bei weitem kompensiert werden, eine nachhaltige Intensivierung der landwirtschaftlichen Produktion wird damit möglich. Tephrosia candida und Leucaena glauca als Heckensystem auf Terrassen zur Reduzierung des Bodenabtrags sowie Mulchverfahren, die eine ganzjährig möglichst hohe Bodenbedeckung gewährleisten, stellen dafür potentielle Möglichkeiten dar. Ziel der Arbeit war es daher, das Nutzungspotential der Pflanzen Tephrosia candida und Leucaena glauca als Erosionsschutzmaßnahme zur Gewährleistung der langfristigen Nutzung landwirtschaftlicher Nutzflächen in Hanglagen zu bestimmen.

Um die dargestellten Maßnahmen in der Praxis umsetzen zu können, werden einfache Vermessungsgeräte zur Bestimmung von Isohypsen und Hanggefälle sowie die Bedeutung traditioneller Formen der gemeinschaftlichen Arbeit vorgestellt. Ferner eine Kombination von nachhaltigen Landnutzungsystemn und Waldbewirtschaftung.

Die Untersuchung ist auf eine ethnische Minderheit gerichtet, die selbst nach vietnamesischem Standard als besonders arm gilt. Eine Erweiterung und Vertiefung derartiger Studien bilden deshalb eine der Voraussetzungen für eine sozial gerechte und friedliche Entwicklung. Sie helfen außerdem, die Kenntnisse, über besonders arme Teile der Gesellschaft, zu erweitern. 


\section{Summary}

The mountainous areas of Vietnam cover $3 / 4$ of the national territory. In these areas, the socio-economic development is based on the high exploitation of the available natural resources such as land and forests for agricultural production and forestry. In process of economic improvement the farmers are still confronted with many challenges especially land issue as result of increasing land degradation. This is due to serious soil erosion exacerbated by the natural conditions such as very steep land, heavy and concentrates rainfall. The Misuse and overuse by farmers give favourable condition for land degradation. The consequence of increasing land degradation reduces crop yields and deteriorates living standard and food security for people lives there. Therefore, in order to improve living standard and food security situation for the farmers in the mountainous areas, a shift toward sustainable land use systems is required in the mountainous areas.

The overall objective of the study was to explain the level of soil erosion and its impact on land degradation process as well as change in the level of crop yields. Also, ist aims to understand the situation of soil erosion control and land rehabilitation measures by the farmers in the mountainous areas under the traditional systems of shifting cultivation and the new model of Agro-forestry with mulch. The specific objectives of the study were:

- To understand the socio-economic status of the farmers in the study area especially concern toward land resource issues.

- To assess soil fertility at different points during different land use cycles and different land use systems.

- To assess soil erosion level due to rainfall and establish the relations to land degradation as well as crop yields at different points during crop cycle and different land use systems.

- To determine the land use type that is more sustainable to extent to farmers in the mountainous areas in Babe district of Vietnam.

The study area is representative for the mountainous areas of North Vietnam with serious soil erosion problem and high available land resource potential. There exists different plots have been set up: (1) shifting cultivation such as plot with forest. (2) first year cultivated land after slash and burn (3) fourth year cultivated land after slash and burn (4) natural fallow land and (5) plot with degraded land (Imperata Cylindrica) (6) agroforestry with mulch. Based on these criteria Babe district was choosen as the study area. The field 
study was done at the start of the rainy season (April) untill the end of the season (October) during 2001.

In order to achieve the objectives of the study, farm and family survey was carried out using standardized questionaire and Participartory Rural Appraisal (PRA) tools to collect the information of the current socio-economic development situation in the area. In order to assess the soil erosion level due to rainfall, an experiments were set for different plots of shifting cultivation systems and agroforestry with mulch system. Measurement of soil erosion level and soil fertility lost during one year was done. Soil samples were taken from experimental plots at two different depth levels and different slope locations (0-30 $\mathrm{cm}, 31-60 \mathrm{~cm})$. The following soil- and nutrient analyses in the laborary have been carried out: - $\mathrm{Nt}, \mathrm{Ct}$ and $\mathrm{P}_{2} \mathrm{O}_{5}$ after Bray

- Exchangeable cations: $\mathrm{Ca}^{2+}, \mathrm{Mg}^{2+}, \mathrm{Na}, \mathrm{K}, \mathrm{Fe}^{2+}, \mathrm{Al}^{3+}$ and $\mathrm{Mn}$

- $\quad \mathrm{pH}$ value and soil content of $\mathrm{Ca}^{2+}, \mathrm{Mg}^{2+}, \mathrm{Na}, \mathrm{K}, \mathrm{Fe}^{2+}, \mathrm{Al}^{3+}, \mathrm{Mn}, \mathrm{Zn}, \mathrm{Cu}, \mathrm{Pb}, \mathrm{S}, \mathrm{Cr}$, $\mathrm{Cd}, \mathrm{Co}, \mathrm{Mo}$ and $\mathrm{Ni}$

In addition, the yield of crops in the plots were measured in order to assess the effect of soil erosion on crop yields on sloping lands.

The socio-economic analysis shows that generally, income from agriculture and forestry contributes more than $90 \%$ of family income. This means that farm activities play an important role to the income of family. Family income was found to have close relationship with land size in general. Family with larger farm size have higher family income. Therefore, the socio-economic analysis indirectly shows the important role of land resources in improving the living standard and food security situation for the farmers in the area. The typical cropping system of the farmers in the study area (Tay ethnic) is mainly monoculture growing maize, cassava, paddy rice and upland rice. On the sloping areas the crop yields decreases over time due to increasing of land degradation. However, it should be noted that there are some farmers (34\%) who do not know the causes leading to soil erosion, and the higher percentage of farmers in the area did not care about the dangers and consequences of soil degradation (62\% of total farmers did not apply any soil erosion control measures). The rapid population growth, increasing human needs and increasing land degradation due to natural conditions as well as human misuse have been leading to food deficits in the area in the past. Therefore, in order to overcome food deficit problems, the natural fallow period was reduced from 5 - 8 years before into 2 - 3 years currently. In addition with the extension of agricultural land from forests may lead 
to increase degradation land process and resulting to the environmental condition in the whole area.

Soil erosion is serious during the rainy period from April until end of September. The results of soil erosion measurements show that during the rainy season in the plots of first year after slash and burn a soil loss of 43 tons/ha due to soil erosion and this figure increases to 58,3 tons/ha in the plot at fourth year after slash and burn. While in the plots of agroforestry and mulch only 3.7 tons of soil loss per ha was recorded during this period. Soil erosion causes land degradation. The highest soil loss due to erosion in year fourth after slash and burn is equivalent to loss of $1234 \mathrm{~kg}$ organic matter, $82 \mathrm{~kg} \mathrm{~N}, 1468$ $\mathrm{kg} \mathrm{K}, 82 \mathrm{~kg} \mathrm{P}, 1035 \mathrm{~kg} \mathrm{Mg}$ and $97 \mathrm{~kg} \mathrm{Ca}$ per ha per year. To replace or purchase the amount of nutrient elements lost by erosion, farmers need to spend approximately $9,000,000$ VND per year to buy fertilizer. This expense seem will increase year after year if current use of land with the traditional shifting cultivation method continues. The yield of maize cultivated on the plot in the fourth year after slash and burn is only $1 / 3$ as compared with the yield of maize on the plot of first year after slash and burn due to high level of soil erosion.

The examination of soil samples shows that $\mathrm{pH}$ value and base saturation in the area clearly decreased after cultivation for few years. The $\mathrm{pH}$ value decreased from 6.5 in the plots of the first year after slash and burn to 4.4 in the plot cultivated of fourth year due to the $\mathrm{V}$-value decreased from $99.6 \%$ in first year to $28.9 \%$ in fourth year cultivated land. The degradation of soil from the first to the fourth year of maize cultivation is indicated clearly by a decrease of organic matter, total nitrogen content and the cations exchange capacity between forestland and first year after slash and burn or between the first year and the fourth years after slash and burn. This change is due to the different level of soil erosion in the plot of first year to fourth year cultivated land. The availability of $\mathrm{P}$ content after Bray in both soil depths (0 to $30 \mathrm{~cm}$ and 31 to $60 \mathrm{~cm}$ ) were very low especially in the plots that was used for long time and tended to decrease fast from the first year to the fourth years after cultivation. This indicates that land becomes degraded fast after few years cultivation. The reason lies in the fact that partly available $\mathrm{P}$ is lost through erosion process and also due to iron and aluminum enrichment at low $\mathrm{pH}$ values leading to reduce phosphorus availability through the irreversible phosphate fixation. Therefore, long time use of land without any soil conservation measures (soil erosion control) soil degradation leads to fallow and unusable grassland (Imperata Cylindrica). 
In this study the agroforestry with mulch indicates the high improved (compared with traditional fallow system) soil erosion control and avaible rehabilitating methods for the sloping lands. Since it reduces soil erosion during the cultivated periods (lowest soil erosion level as compared to the others cultivated plots) in addition to increasing soil fertility when compared with the degraded plots of Alang-alang. Therfore, in order to maintain and improve soil fertility, the agroforestry system (combination between agriculture, forests and hedging of Tephrosia candia and Leucaena glauca, and using their leaves for mulch ) seems to lead to a subtainable land use on sloping areas.

The study also shows that the agroforestry system with using Tephrosia candia and Leucaena glauca (plant at isolines) has a high potential to substitute the traditional shifting cultivation system because this system increases land use rate $(\mathrm{R})$ with increases of soil fertility on the slopping fields and resulting increases in crop yields. Therefore, this model should be extended and developed for the whole region.

In order to achieve the objective of development and extension the agroforestry with mulch to the whole region, which has high benefits in terms of environmental and economic aspects, there is a need to train farmers in simple techniques of setting and using the Agro-forest with mulch model that was presented in the thesis.

Further more, the result of the research also contributes to overall food and poverty reduction effort by quantifying what would be gained in term of yield gain if these measures are probably implemented in the mountainous areas, where mainly ethnics and poor people live. Therefore, the result of the study not only establish es sustainable land use alternative, but also contributed to the equal development between the mountainous areas and the lowland areas. 


\section{LITERATURVERZEICHNIS}

A. G. BodEN (1994): Bodenkundliche Kartieranleitung. 4. Auflage .Hannover.

ACHTNICH W (1986): Landnutzung, Bodenfruchtbarkeit und Pflanzenproduktion in den Tropen und Subtropen, Heft 12- Göttingen beiträgt zur Land -und Fortwirtschaft in den Tropen und Subtropen.

Agassi, M. (1996): Soil Erosion, Conservation and Rehabilitation. New York-BaselHong Kong

AgBoola, A. A (1975): Problems of improving soil fertility by the use of green manuring in the tropical farming system. FAO Soils Bulletin No.27

AID (1993): Erosionsschäden vermeiden -Bonn, S32.

ALEXANDER, M. (1980): Effects of acidity on micro-organisms and microbial processes in soil.- In: HutChINSON, T.C. \& M. HAVAS (Hrsg.): Effects of acid precipitation on terrestrial ecosystems- Plenum Press, New York, S. 363-380)

ANDRIESSE, J. P \& KoOPMANS (1984): A Monitoring study on nutrient cycles in soils use for shifting cultivation under various climatic conditions in tropical Asia. I. The influence of simulated burning on form and availability of plant nutrients. - In Agriculture, Ecosystems and Environment, 12(1984) 1-16.

Andriesse, J. P. \& SchelhaAs, R. M. (1987a): A Monitoring Study on Nutrient Cycles in Soils Used for Shifting Cultivation under Various Climatic Condition in Tropical Asia. II. Nutrient Stores in Biomass and Soil-Results of Baseline Studiens. -In: Agriculture, Ecosystems and Environment, 19 (1987) 285-310.

Andriesse, J. P. \& SchelhaAs, R. M. (1987b): A Monitoring Study on Nutrient Cycles in Soils Used for Shifting Cultivation under Various Climatic Condition in Tropical Asia. III. The Effects of Land Clearing Through Burning on Fertility Level. -In: Agriculture, Ecosystems and Environment, 19 (1987) 311-332.

ANDRIESSE, J. P. (1987): Monitoring project of nutrient cycling in soil used for shifting cultivation under various climates conditions in Asia. Royal Tropical Institute, Amsterdam, S. 1-140.

Auerswald, K. ( 1993): Relief Boden Paläoklima- Bodeneigenschaften und Bodenerosion. Gebrüder Bornträger. Berlin. Stuttgart.1993

Auerswald, K. (1991b): Onsite- und Offsite- Schäden durch Bodenerosion. - Boden nutzung und Bodenfruchtbarkeit Bd. 3, Verlag Paul Parey, Hamburg und Berlin , S.75-82

AuERWALD, K. (1985): P-und K- Verluste durch Wassererosion nach der Wintergerstenund Rapsernte. -Kali-Briefe (Büntehof) 17

BABALOLA, O. \& CHHEDA, H. R. (1975): Influence of crops and cultural practice on soil and water loss from a western Nigerian soil. Ghana Journal of Science 15.

BABALOLLA, O. \& CHHEDA, H. R. (1975): Influence of crops and cultural practice on soil and water loss from a western Nigerian soil. Ghana Journal of Science 15: 9395.

BARBER, S.A (1995): Soil nutrient bioavailability: a mechanistic approach, NewYork

BACHE, B. W. \& HeAthcote, R. G. (1969): Long-term effects of fertilizers and manure on soil and leaves of cotton in Nigeria. Expl. Agric. V. 5: 241-247.

BAhrenberG, G., Giese, E. \& NiPPER, J. (1990): Statistische Methoden in der Geographie 1.-Stuttgart, 233S.

BARSCH, H.; BILlwitz, K \& BORK H-R. (2000): Arbeitsmethoden in Physiogeographie und Geoökologie, Stuttgart :Klett-Perthes. $612 \mathrm{~S}$.

BARROW, C. J. (1991): Land degradation: development and breakdown of terrestrial environments. Cambridge University Press, Cambridge, 295 S. 
BECKER, B. (1994): Integrierter Erosionsschutz in den Anden - Journal of Agriculture in the Tropics and Subtropics. Der Tropenlandwirt - Beiheft Nr.50.

BECKER. K. W. et al. (1999): Materialien zur Vorlesung: Aspekte und Grundlagen der Bodenkunde - 24.Aufl., Göttingen: Eigenverlag, 1999

Bergeret, A. (1984): Ökologisch lebensfähige Produktionssysteme Illustration im Bereich der Landwirtschaft- In: Rottach, P.(Hrsg.): Ökologischer Landbau in den Tropen. Verlag C.F. Müller, Karlruhe, 107-139.

BMZ (2002): Umwelt-Entwicklung- Nachhaltigkeits- Entwicklungspolitik und Ökologie

Boardman, J., Foster, I. D. L. \& Dearning, J. H. (1990): Soil erosion on agricultural land. - British Geomorph. Res. Group Symposium Ser., Chicheter, 687 S.

BODENKUNDLICHES PRAKTIKUM (1995): Eine Einführung in pedologisches Arbeiten für Ökologen, insbesondere Land- und Forstwirte, und für Geowissenschaftler-2. neubearbeite Auflage- Blackwell wissenschafts- Verlag Berlin. Wien -

BöHM, P. (1995): Bodenerosion und pedohydrologische Gebietsvarianz im Einzugsgebiet des Karabalcik (Türkei). Diss im Geogr. Inst. Univ. Göttingen 197 S.

BoHNET, M. (2001): Stellenwert der Ländlichen Entwicklung in der deutschen Entwicklungszusammenarbeit. In Entwicklung ländlicher Raum-Schwerpunk: Neue Konzepte zur Welternährung. Helf 1/2001.

BoRK, H. R. (1988): Bodenerosion und Umwelt. Verlauf, Ursachen und Folgen der mittel alterlichen und neuzeitlichen Bodenerosions- Landschaftsgenese Landschaftsökologie, Bd.13 249 S.

Borst, H. L. \& WoOdDBuRn, R. (1942): The effect of mulching and methods of cultivation on runoff and erosion from Muskingum slit loam. Agricultural Enginneering 23: 19-22.

BRAUNS, T. \& SchOlZ, U. (1997): Shifting cultivation - Krebsschaden aller Tropenländer ? Geographische Rundschau 49 (1997) H.1, S.3-10.

Breburda, J.,(1983): Bodenerosion und Bodenerhaltungs- DLG- Verlag , Frankfurt (Main)

BRECKLING, B.\& BIRKENMEIER, P. (2000): Landnutzungsalternativen im Regenwald Praxis und Theorie der Ökosystemkonformen Landnutzung- Peter Lang Europäischer Verlag der Wissenschaften(Sig F2002, B 31)

Brown, R. LESTER \& WOLF, C. ERWARD (1985): Erosion der Tod der Böden oder Die schleichende Gefahr für die Weltwirtschaft. Biologische Schutzgemeinschaft Hunte Weser-Ems Naturschutzverband Niedersachsen- Worldwatch Paper 60).

BRYAN, R. B. (1987): Processes and significance of rill development. - Catena Suppl.8: 116.

BÜHL, A. \& P. ZÖFEL, (1996): SPSS für window 10.0: Praxisorientierte Einführung in die moderne Datenanalyse, Bonn(u.a) Addison-Wesley-Lonhmann,1996. 670 S.

BUI, Q. T (1990): Some Problems of soil on slash-and-burn cultivation in North-western region and the direction of its utilization. Ph.D. Thesis. Hanoi University, Hanoi (Vietnamese).

Chao Shang \& Holmt (1997): Soil Science. Vol. 162, No 11, Printed in U.S.A.

Cuevas , V. C. \& Duma, J. C. (1998): Nutrient Balance of Three Different Land Uses in the Forest Reserve of Mt. Makiling, Philippines (S.11-62) in : Land degradation and Agricultural sustainability: Case Studies from Southeast and East Asia Edited by Aran Patanothai - Regional Secretariat- Khon kaen, Thailand.

DE Rouw, A. (2003): Weed Infestation and Soil Erosion Resulting from the Breakdown of the Slash and Burn Cultivation System in: ANTHONY S. R. JuO \& ANDREW 
MANU (1996): Chemical dynamics in slash-and-burn agriculture -In: Agriculture Ecosystems \& Environment S.49-60.

Derpsch, R., Roth, C. H., SidiRAS, N. \& KÖPKE, U. (1988): Erosionsbekämpfung in

Parana, Brasilien: Mulchsystemme, Direktsaat und Konservierende Bodenbearbeitungs- Schriftreihen der GTZ, Nr. 205, TZ- Verlagsgesellscharf, Rossdorf.

Do, D. S. (1994): Shifting cultivation in Vietnam: Its social, economic and environmental values relative to alternative land use. IIED Forestry and Land Use Series No.3.IIED.

DoAn, T N. (1990): Xoi mon va bien phap chong xoi mon tren lanh tho Vietnam. Hanoi

Doleschel, P. \& Heissenhuber, A. (1991): Externe Kosten der Bodenerosion. Landwirtschaftliches Jahrbuch, 68Jhrg., Helft 2, S.187-209

DOLESCHEL, P. (1993): Fruchtfolgeoptimierung zur Einhaltung von Bodenabtragstoleranzen.- Dissertation, Lehrstuhl für Wirtschaftslehre des Landbaues der Technischen Universität Münschen

Doppler, W. \& BAUER, S. (2001): The Socio-Economic Impact of Soil Degradation on Upland Farming Systems In West Sumatra, Indonesia- Farming and Rural Systems Economics- Volume 101- Wissenschaftsverlag VAUK.

DoppleR, W. (1991): Landwirtschaftliche Betriebssysteme in den Tropen und Subtropen. Stuttgart 1991.

Doppler, W AT AL (2000): Farming and Rural Systems Approaches, Hohenheim, Stuttgart: Published Lecturing Material.

DRECHSEL, P. (1992): Beziehungen zwischen Standort, Ernährungsleistung und Wuchsleitung von Teak (Tectona grandis L.P) im humiden Westafrika. - In Bayreuther Bodenkundliche Berichte, Bd. 31.

Duley, F. L. \& Russel, J. C. (1943): Effect of stubble mulching on soil erosion and runoff. Proceedings of the Soil Science Society of America 7: 77-81.

EgGER, K. (1989): Ökolandbau in den Tropen. In: Ökologie und Landbau, IFOAM 70, S.18-21.

EgGer, K; KorUs U. (Hrsg.) (1993): Öko-Landbau in den Tropen-Stiftung Ökologie \& Landbau C.F.Müller Verlag.

EITEL, B. (1999): Bodengeographie - Westermann GmbH, Braunschweig. 244 S.

ELLENBERG, H. (1984): Entwicklung ohne Rückschläge -Schriftenreihe der GTZ, Nr. 156, TZ- Verlagsgesellschaft, Rossdorf.

El-Swaify, S. A., E. W. Dangler, C. L. Armstrong (1982): Soil Erosion by water in the tropic College of Tropical Agriculture and Human Resources, University of Hawaii. - In: Morgan, R. P. C. (1999): Bodenerosion und Bodenerhaltung, Stuttgart 1999, 236S

Erger, K./Korus, U. (1995): Öko-Landbau in den Tropen- Traditionelle und moderne Bespiele aus Ostafrika- Alternative Konzepte- Buchreihe der Stiftung Ökologie \& Landbau, Bad Dürkheim- C.F. Müller Verlag, Heidelberg

EvAn, C. E. \& E. J. KAMPRATH (1970): Lime responses as related to percent aluminum saturation, solution aluminum, and organic content. Soil Sci. Soc. Am. Proc. 34:893-896.

FAO (1995): The Conservation of Lands in Asia and the Pacific (CLASP), Rome

FAO (1996): Workshop on Forestry and Agro-forestry on Sloping Land of Northern Vietnam.

FAO( 2001): Farming Systems and Poverty Improving farmers' livelihoods in a changing world 
FiedLeR, H. J. (2001): Boden und Bodenfunktion in Ökosystemen, Landschaften und Ballungsgebieten. Forum eipos -Band 7- Experte verlag

FINCK, A. (1963): Tropische Böden- Einführung in die bodenkundlichen Grundlagen tropischer und subtropischer Landwirtschaft - Verlag Paul Parey. Hamburg und Berlin

Flaig, W., Nagar, B. H., Söchtig, H. und Tietjen, C. (1978): Organic materials and soil productivity. Rom, FAO Soils Bull. No.35

FrANKE, G. (1970): Probleme der Intensivierung der Pflanzenproduktion in Entwicklungsländer in: Probleme des Aufbaues einer progressiven Landwirtschaft in den Entwicklungsländern. Institut für tropische Landwirtschaft und Veterinärmedizin der Karl-Marx-Universität Leipzig, S123141.

FrANKE, G. (1981): Nutzpflanzen der Tropen und Subtropen. 3. Auflage, Bd. 1 und 2, Leipzig, Hirzel, 441 bzw. 398 S.

FungameZA, D. B. (1993): Agro-forestry and Eco-farming Practices for Soil Conservation in Kigoma Tanzania. Göttingen Beiträge zur Land- und Forstwirtschaft in den Tropen und Subtropen, Helft 63.

Gabriels, D. \& BoodT, M. (Hrsg.) (1980): Assessment of erosion.- Chichester, 563 S.

GEROLD, G. (1979): Untersuchungen zum Naturpotential in Südostsizilien im Hinblick auf ihre Bedeutung für die agrare Landnutzung. Jb. Geogr. Ges. Hanover, Bd.186, Hanover, 273S.

GEROLD, G. (1986a): Vegetationsdegradation und fluviatile Bodenerosionsgefährdung in Südbolivien. Habilitationsschrift, Hanover 1986, 404 S.

Gerold, G., (1986b): Klimatische und pedologische Bodennutzungsprobleme im ostbolivianischen Tiefland von Santa Cruz. -In: Jahrbuch der GGH, Hanover, S.69-162.

GEROLD, G. (1988): Zur Anwendung von Schätzmodellen der Abspülresistenz tropischer Böden bei Neulanderschließungen am Beispiel der äußeren Tropen- Jb. Geogr. Ges. Hanover (1988) S. 161-188.

GEROLD, G. (1989): Vegetationsdegradation und fluviatile Bodenerosionsgefährdung in Südostbolivien- Habilitationsschrift, 404S. (im Druck)

GEROLD, G. (1991a): Klimatische und pedologische Bodennutzungsprobleme in tropischen Waldgeschenschaften (Bolivien).- In: Giessener Beiträge zur Landund Forstwirtschaft in den Tropen und Subtropen, Heft 60, Göttingen, S.91-110.

GEROLD, G. (1991b): Nutzungseingriffe in tropischen Waldgesellschaften und deren pedoökologische Folgen (Bolivien). In: Giessener Beiträge zur Entwicklungsforchung. Reihe I(Syposien), 19, Tropeninstitut Gießen, S.25-40.

GEROLD, G. (1991c): Human impact on Forest Ecosystems and Soil Deterioration in Tropical Bolivia. - In ERDELEN, W., MÜLLER, P. ISHWARAN, N.: Tropical Ecosystems. Markgraf Scientific Books, Weikersheim, S. 107-120.

GEROLD, G. (1997): Bodendifferenzierung, Bodenqualität und Nährstoffumsatz in ihre Bedeutung für die Waldrehabilitation und landwirtschaftliche Nutzung in der Ostregion der Elfenbeinküste. In: Göttinger Geographische Abhandlungen, 100, S.147-148

GEROLD, G. (2002): Geoökologische Grundlagen nachhaltiger Landnutzungssysteme in den Tropen- Geographische Rundschau 54 Mai, 2002.

Ghosh, S. P., Mohan Kuma, B., Kabeerathumma, S. \& Nair, G. M. (1986): Productivity, Soil Fertility and Soil Erosion under Cassava Based Agro-forestry Systems. In: Agro-forestry System 8.S.229-249. 
GLASOD, 2000: Global Assessment of Soil Degradation aus http:/edoc.huberlin.de/dissertationen/agrar/morgenroth-silvia/HTML/morgenroth-ch3.html:

HA, NGuyen T; SiEM, T \& PHIEN, T. (1998): Identification of Erosion Factors and Evaluation of Potential soil loss on Sloping lands of Vietnam S.149-163 - In: Sustainable farming on sloping lands in Vietnam (Research Results 1990-1997), Agriculture publishing house Hanoi.

Hadley, M. \& LANLy, J. P. (1983): Tropical forest ecosystems: identifying differences, seeking similarities, Nature and Resources, vol. 19, S. 2-19.

HARRACH, T. (1975): Die Ertragsfähigkeit von Lößböden unterschiedlichen Erosionsgrades. Lanw. Forschung Bd. 28

HARRACH, T. (1981): In BOGUSLAWSKI, E. Von (1981) Ackerbau: Grundlagen der Pflanzenproduktion. Frankfurt, DLG- Verlag, Kap. 5: 127-169.

HARTGE, K,H \& HoRN, R. (1991): Einführung in die Bodenphysik- Stuttgart: Enke

HATCH, T. (1982): Shifting cultivation in Sarawak- A review, Technical paper No. 8, Soil Division Research Branch, Department of Agricultural, Sarawak, Indonesia.

Heisenhuber, A. \& SCHMidTlein, E. A. (1987): Ökologische und ökonomische Probleme der Bodenerosions-Vorträge der XXV. Weihenstephaner Hochschultagung am 10.Juli 1987 in Weihenstephan, Bayerisches Landwirtschaftliches Jahrbuch, 65. Jahrgang, Sonderheft 1.

HEMMER, H.,R(1999): Wirtschaftliche Systemtransformation und Armutsbekämpfungeine ökonomische Analyse am Beispiel der Sozialistischen Republik VietnamBand 78-Verlag Münster-Hamburg-London

HOANG FAGERSTRÖM, M. H. et al., (2000): Innovations within upland rice-based systems in northern Vietnam with Tephrosia Candida as fallow species, hedgerow, or mulch: net returns and farmers' response. In Agriculture Ecosystems \& Environment - 86 (2000) 23-37.

HoANG, X, T (2000): Kein thuc ban dia cua dong bao dan toc thieu so vung cao-Vien tai nguyen sinh thai va moi truong- Nha xuat ban Ha noi

HoFFMANN G. (1991): Methodenbuch-Band1- Die Untersuchung von Böden- VDLUFAVerlag. Darmstadt.

http://vnexpress.net/Vietnam/Xa-hoi/2002/05/3B9BC34F

http://vnexpress.net/Vietnam/Xa-hoi/2003/03/3B9C5B94/

http://www.bmz.de/infothek/buerger/oekobroschuere.pdf

http://www.bmz.de/infothek/fachinformationen/konzepte/konzept114 asien.pdf

http://www.bmz.de/infothek/fachinformationen/spezial/spezial066/spezial066 14.html

http://www.mekonginfo.org/mrc en/doclib.nsf/0/1DE552D39A4E73F347256CED00300

B7F/\$FILE/FULLTEXT.html

http://www.mekonginfo.org/mrc en/doclib.nsf/0/3390086C8EE15ABC47256CE5003089 F7/\$FILE/FULLTEXT.html

http://www.mekonginfo.org/mrc en/doclib.nsf/0/840A97D821C0725547256CF5003556

D9/\$FILE/FULLTEXT.pdf

http://www.mekonginfo.org/mrc en/doclib.nsf/0/A78B0DE410AD149A47256D6600360

D5B/\$FILE/FULLTEXT.html

http://www.mekonginfo.org/mrc en/doclib.nsf/0/C5C949362A3770A047256D6E002756 84/\$FILE/FULLTEXT.html

http://www.tropentag.uni-goettingen.de/links/Do_Thi me40cpoE.pdf

http://www.troz.uni-hohenheim.de/research/Thesis/MScEE

http://www.zef.de/zefnews/no11-12-02-dt.pdf

HudsOn, N. W. (1981): Soil conservation. London, Batsford. 
HURI, H \& NUNTAPONG, S. (1983): Agro-forestry improvements for shifting cultivation systems soil conservation research in northern Thailand, Mountain Research and Development, Vol.3, no. 4, pp. 338-345.

JanetscheK, H.(Hrsg), AleXander, C., Georg, G., Werner, S., WolfGang, S. (1982): Ökologische Feldmethoden- Hinweise zur Analyse von LandökosystemenVerlag Eugen Ulmer Stuttgart -1982.

JANOS, D. P. (1980): Mycorrhizae influence tropical succession. Tropical Succession.

JAROSCH, J. \& ZEDDIES, J. (1991): Bodenerosion - Ökonomisch Aspekte von Schäden und Schutzmaßnahmen- In: Bodennutzung und Bodenfruchtbarkeit Bd. 3, Verlag Paul Parey, Hamburg und Berlin, S.99-116

JenNy \& Raychaudhuri (1960): In Nguyen, T, S. \& Thai, P (1999): Upland soil in Vietnam Degradation and Rehabilitation. Agriculture Publishing House, Hanoi 1999

JORDAN, C. P. (1989): An Amazonian rain forest: the structure and function of a nutrient stressed ecosystem and the impact of slash and burn agriculture. Series : MAB. Unesco. Athens, Georgia.

JuLIO, C. \& CASSEL, D. K. (1996): Dynamics of soil physical properties under alternative systems to slash-and- burn. In Agriculture, Ecosystems and Environment - 58 (1996) 39-48.

KAnNT, G. (1983): Gründüngung. 2. verb. Auflage. Frankfurt a.M. DLG-Verlag, 146 S.

Karsten, R. (2000): Pedoökologische Auswirkungen des Brandrodungsfeldbaus in den wechselfeuchten Tropen (Santa Cruz- Bolivien)- Diplomarbeit Göttingen

KELlOGG, C. (1962): Shifting cultivation. In: Soil Science, vol.95 (1963), p. 221-230.

KHYBRI, M. L. (1989): Mulch effects on soil and water loss in maize in India. In W C Modenhauer, N W Hudson, T C Sheng and S W Lee (eds), Development of conservation farming on hillslopes. Ankeny IA, Soil and Water Conservation Society: $195-8$.

Kirkby, M. J. \& Morgan, R. P. C. (1980): Soil erosion.- Chichter, 312 S.

Kleinman, J. A., Bryant R., Pimentel, B. \& D. (1996): Assessing Ecological Sustainability of Slash-and- burn Agriculture through Soil Fertility Indicators In Published in Agron. J.88:122-127

Klinge, H., Rodrigues, E., Brunig, E. \& FitTKaU, E. J. (1975): Biomass and structure in a Central Amazonian rain forest. In: Golley, B. \& Medina, E (1975)(Hrsg.): Tropical ecological systems, Springer 179-222.

KÖNIG, D. (1992): Erosionsschutz in Agroforstsystemen. Möglichkeiten zur Begrenzung der Bodenerosion in der kleinbäuerlichen Landwirtschaft Rwandas im Rahmen standortgerechter Landnutzungssysteme. Maiz. (Maizer Geographische Studien, H.37).

KÖNIG, D. (1996): Bodenschutz in tropischen Agrofostsystemen. Mitt. Der Deutschen Bodenkunlichen Ges. 79 (1996) S: 399-402.

Kotschi, J., Waters-Bayer, A., Adelheim, R., Hoest, U. (1989): Eco-farming in agricultural development. - Verlag Josef Margraf, Weikersheim.

KREBS, J. E. (1975): A comparison on soil under agriculture and forests in Saan Carlos/ Costa Rica. In : GOLLEY und MEDINA (1975) : 381-390.

KRETZSCHMAR, R. (1990): Schutz vor Wassererosions- In: Blume, H-P.(Hrsg): Handbuch des Bodenschutzes. Ecomed, Landsberg (lech) 530-539.

KretzsCHMAR, R. (1996): Kulturtechnisch -bodenkundliches Praktikum- Ausgewählte Labor- und Feldmethoden, Bd.2 2- Chemische Verfahren der Standortuntersuchung. Skript der Universität Kiel.

KURTHS, K. (1997): Private Kleinbetriebe in Vietnam, Saarbrücken. 
Kuypers, H., Mollema, A., Topper, E. (1987): Erosion control in the tropics.- Agrodok 11, Agromisa, Wageningen, Niederlande

KuYPers, H.; MOllemA, A.; TOPPER, E., (1987) : Erosion control in the tropics-Agrodok 11, Agromisa, Niederlander

LAND LAW OF VIETNAM, 1993

LAL, R. (1976): Soil erosion problems on an alfisol in Western Nigeria and their control. IITA Monograph No.1.

LAL, R. (1991): Myths and Scientific Realities of Agro-forestry as a Strategy for Sustainable management in the Tropics - Advances in Soil Science, Vol.15, Springer-Verlag, New York, 91-137.

LAL, R. (Hrsg.), (1988): Soil erosion research methods - Wageningen. 244 S.

Lal, R., Hall, G. F. \& F. P. Miller (1989): Soil degradation: 1. Basic process.- In: Land degradation and Rehabilitation, Bd.1

LAmotTe, M. (1975): The Structure and function of a tropical savannah ecosystem. In: Golley, B. \& MedinA, E (1975)(Hrsg.): Tropical ecological systems, Springer179-222.

LANDON, J. R. (1984): Booker tropical soil manual. London, 450S.

LAUER, W. (1956): Vegetation Landnutzung und Agropotential in EL Salvador. Schriften des geographischen Instituts der Universität Kiel, Bd. 16:(1).

LAUER, W. (1998) : Klimatologie- Das Geographische Seminar - Westermann

LE T. C. (1995): Rehabilitation of degraded land in Midland of Northern Vietnam. In: Some issues of human ecology in Vietnam, (ed.) LE, T. C. and A. TERrY RAMBO. Hanoi: Agricultural Publishing House. (In Vietnamese)

LE, V., K. \& DoAn, T, N (1985): Chong xoi mon rua troi dat o dat Phu quy, tap san KHCN 1985, Hanoi

LESER, H. (1977): Feld- und Labormethoden der Geomorphologie- de Gruyter-Verlag. Berlin/New York

LoRENZ, S. (1990): Möglichkeiten und Grenzen des Alley Cropping in der Entwicklung der Bodennutzung in Africa.- Der Tropenlandwirt, Beiheft Nr.44, EkopanVerlag, Witzenhausen.

Maene, L. M., Thong, K. C., Ong, T. S., Mokhtaruddin, A. M. (1979): Surface wash under mature oil palm. In E Pushparajah (ed.), Proceedings, Symposium on Water in Malaysian agriculture. Kuala Lumpur, Malaysian Society of Soil Science: 203-16.

Maydell, H. J. (1986): Agroforstwirtschaft in den Tropen und Subtropen- In: Rehm, S(Hrsg.): Grundlagen des Pflanzenbaues in den Tropen und Subtropen. Eugen Ulmer Verlag, Stuttgart, 169-190.

MARKUSSEN, M (2000): Pedoökologische Folgen des mennonitischen Landnutzungssystem im Oriente Boliviens (Departamento Santa Cruz)-Diplomarbeit

McGrath (1987)- In: Breckling, B.\& P. BirkenmeIER(2000): Landnutzungsalternativen im Regenwald - Praxis und Theorie der Ökosystemkonformen Landnutzung- Autochthone Anbauweisen und die Brandrodungsproblematik (S.135-141)-Peter Lang - Europäischer Verlag der Wissenschaften.

MeliczeK, H. (1999): Sozialökonomik der landwirtschaftlichen Entwicklung

MestMACHER, F. (1989): Rezente Landschaftsveränderungen durch Landnutzung und Bodenerosion im Kenia - Dissertation

MOHR \& VAN BAREN (1954): in HÖFNER \& MOLL (Hrg) (1990) - Bodenfruchtbarkeit und Pflanzenernährung - Giessener Beiträge zur Entwicklungsforschung- Reihe 1, (Symposien)Band 18. 
Mokhtaruddin, A. M. \& Maene, L. M. (1979): Soil Erosion under different crops and management practices. Proceedings, International conference on agricultural engineering in national development. University Pertanian, Malaysia, Paper No. 79-53.

MoLL, WALTER L.H (1982): Taschenbuch für Umweltschutz: ökologische Information.

MöLlER, H. (1979): Untersuchungen zum Einfluss der Besenheide auf die Aktivität von Enzymen im Böden, dargelegt am Beispiel des Sandstrandes Ostseeküste Schleswig- Holsteins. Flora 168: 320-328

Morgan, R. P. C. (1980): Implications - In: Kirkby, M.J., Morgan, R.P.C (Hrsg.): Soil Erosion. John Wiley \& Sons, Chichester, 303-306.

Morgan, R. P. C. (1999): Bodenerosion und Bodenerhaltung, Stuttgart 1999, 236 S.

Morgan, R. P. C., Martin, L., Noble, C. A. (1986): Soil erosion in the United Kingdom: a case study from mid-Bedfordshire. Silsoe College Occasional Paper No. 14.

MÜLLER- SÄMANN, K. M. (1986): Bodenfruchtbarkeit und Standortgerechte Landwirtschaft- Maßnahmen und Methoden im Tropischen PflanzenbauSchriftenreihe der GTZ, Nr.195, Eschborn.

Munsell SOIL COLOUR CHARTS (1990): Standard soil colour charts

NAIR, P. K. R. (1993): An Introduction to Agro-forestry, S 55-74, 268-300 and 74-79, Kluwer Academic Publishers, Dordrecht, The Netherlands.

NAir, P. K. R., \& Muschler, R. G. (1993): Agro-forestry. In: L. PANCEL (Ed.): Tropical Forestry Handbook Vol. 2. Berlin, Heidelberg, New York, London, Paris, Tokyo, Hong Kong, Barcelona, Budapest 1993, S. 997-1057.

National Assembly the Socialist Republic of Vietnam (1993): Land law of Vietnam, National Politics Publishing House, Hanoi, 1993.

NeugebaueR, B(1986): Der historische Wandel kleinbäuerlicher Landnutzung in Oxkutzcab/Yucatan : ein Beitrag zur Entwicklung sozial und ökologisch angemessener land- und forstwirtschaftlicher Methoden in den feuchten Tropen Mexikos

Nguyen Tu Sien \& Thai Phien ( 1999): Upland soil in Vietnam- Degradation and Rehabilitation - Agricultural publishing house. Hanoi

NGuYen, T, S. \& THAI, P. (1992): Organic matter recycling improvement of sloping lands in Vietnam. Annual Meeting of the IBSRAM'S ASIALAND Network on Sloping lands Management for Sustainable Agriculture, Hanoi

NguYen, T, D. \& D, T. L.AN (1998): Research results on sustainable cultivation practices on sloping lands in the mountain areas of Northern Vietnam.

Nguyen, T., S \& Thai, P (1991): Bodenerosion und Degradation in Vietnam. Band 3, Agricultural publishing house. Hanoi

Nguyen, X. Q. (1994): Sustainable land use of sloping land, Agricultural Publishing House (in Vietnamese). Hanoi

NiEDERSÄCHSISCHES MiNISTERIUM FÜR ERNÄHRUNG, LANDWIRTSCHAFT UND FORSTEN (1989): Erhaltung des Bodens durch Erosionsschutz- Bremen.

Nye, P. H. \& GReEnland, D. J. (1960a): The Soil under shifting cultivation. Comm. Bur. Soil Sci. Techn. Commun. No. 50.

Nye, P. H. \& GREENLAND, D. J. (1960b): The Soil under shifting cultivation. Farnham Royal, Commonwealth Bureau of Soil, Techno. Communication No.51: 156p

NYLE C. B. (1996): Alternatives to slash-and-burn: a global imperative. In: Agriculture Ecosystem \& Environment 58(1996) 3-11.

OthiENO, C. O. (1978): An assessment of soil erosion on a field of young tea under different soil management practices. In: Soil and Water conservation in Kenya. 
Institute of Development Studies University of Nairobi, Occasional Paper No. 27: 62-63.

OvuKa, M. (2000): Soil nutrient changes along slope transects in Murang a District- In: Effects of soil Erosion on Nutrient status and soil productivity in the central highlands of Kenya- Distribution: Earth Sciences Centre, Göteborg, Sweden Oxford. Edinburgh. Boston. London. Melbourne. paris.ykomaha

PAGEL, H. (1974): Sättigungsgrad und pH-Wert verschiedener tropischer Böden und ihre

Beziehungen $\mathrm{zu}$ anderen Bodeneigenshaften. Beitr. Trop. Landwirtsch.

Veterinärmed. 12 393-407.

PAGEL, H. (1981): Grundlagen des Nährstoffhaushaltes tropischer Böden. VEB Deutscher Landwirtschaftsverlag Berlin, $192 \mathrm{~S}$.

Pagel, H., EnzManN, J. \& Mutscher, H. (1982): Pflanzennährstoffe in tropischen Böden -ihre Bestimmung und Bewertung. VEB Deutscher Landwirtschaftsverlag Berlin. 272S

PAREY, P (1991): Bodennutzung und Bodenfruchtbarkeit- Band 3 : BodenerosionBerichte über Landwirtschaft : Sonderheft ; N.F., 205,. Robert-Bosch-Stiftung $\leq$ Stuttgart $>, 133 \mathrm{~S}$

PAREY, P. (1991): Bodennutzung und Bodenfruchtbarkeit- Band 3: Bodenerosions-Verlag Paul Parey. Hamburg und Berlin.

PAREY, P.(1991): Bodennutzung und Bodenfruchtbarkeit-Band1: Bodenfruchtbarkeit Berichte über Landwirtschaft : Sonderheft ; [N.F.], 203-Robert-Bosch-Stiftung $\leq$ Stuttgart $>$ - Verlag Paul Parey- Hamburg und Berlin, $157 \mathrm{~S}$.

PAREY, P.(1992): Bodennutzung und Bodenfruchtbarkeit- Band 4: HumushaushaltBerichte über Landwirtschaft : Sonderheft ; N.F., 206 Verlag Paul PareyHamburg und Berlin, 179S.

Perter, W. J. \& Neuenschwander (1988): Slash and burn. Moscow, Idaho. University of Idaho Press

Pham, N., D, (1990): Nghien cuu 1 so bien phap chong xoi mon tren dat do Bazan trong che vung Tay nguyen. Luan an pho tien si nong nghiep, Vietnam

PHAN, X., D., et al. (2001): Bao ve moi truong va phat trien ben vung o Vietnam, Ban khoa giao trung uong, lien hiep cac hiep hoi vietnam voi su tai tro cua quy ho tro moi truong toan cau TOYOTA 500. Hanoi

PhIEN, T \& SIEM, N.T (1998): Green manure cover crops for Effective use of sloping lands in Vietnam- Hanoi Publishing House.

Pimentel, D., Harvey, C., Resosudarmo, P., Sinclair, K., Kurz, D., McNair, M., Crist, S., ShPritz, L., FitTON, L.; SAFFouri, R., Blair, R. (1995): Environmental and Economic Cost of Soil Erosion and Conservation Benefits. In: Science, Jg. 267, S. 1117-1123.

Pinstrup-Andersen, P., Pandya-Lorch, R., Rosegrant, M. W. (1997): The World Food Situation: Recent Developments, Emerging Issues, and Long term Prospects. 2020 Vision Food Policy Report, Washington, D.C.: International Food Policy Research Institute (IFPRI).

Preuschen, G. \& Bernath, K. (1983): Die Kunst der Gründüngung. Voraussetzung für Bodenfruchtbarkeit. Leopold Stocker- Verlag, Graz-Stuttgart.

Pimentel, D.(1989) : Ecological Systems, Natural resources, and Food Supplies. In: Food and Natural Resources, edited by Dayid Pimentel and Carl W.Hall, San Diego, Califorlia 92101:Harcourt Brace Jovanovich, p. 1-29. 
PRINZ, D. (1986): Ökologisch angepasste Produktionssysteme. -In: Rehm, S (Hrsg.): Grundlagen des Pflanzenbaues in den Tropen und Subtropen, Verlag Eugen Ulmer, Stuttgart, 115-168.

R. O. ILAO and M. T. DE GUZMAN (2003): The on-site cost of Soil Erosion: The Case of Mapawa Catchment, Lantapan, Philippines In: http://www.mekonginfo.org/mrc en/doclib.nsf/0/6688507611DD471547256D6 50019AA33/\$FILE/ABSTRACT.html

REHM, S. (1986): Handbuch der Landwirtschaft.

ReHM, S. (Hrsg.) (1986): Grundlagen des Pflanzenbaus in den Tropen und Subtropen Handbuch d. Landw. U. Ernährung in den Entwicklungsländern, Bd.3, Stuttgart 1986,478 S.

REINING, LUdGER (1992): Erosion in Andean hillside farming: Characterization and reduction of soil erosion by water in small scale cassava cropping systems in the Southern Central Cordillera of Colombia, Weikersheim.

REVISED-EIJKELKAMP (1997): Standard soil color charts -Agrisearch Equipment

RichteR, G. ( Hrsg.) (1998): Bodenerosion- Analyse und Bilanz eines Umweltproblems, Darmstadt 1998, 236 S.

RICHTER, G. (1965): Bodenerosion. Schäden und gefährdete Gebiete in der BRD.- Forsch. Z.dt. Landeskunde, Bd.152, Bd. 152, Bad Godesberg, 592 S.

RoHrer, J. (1985): Quantitative Bestimmung der Bodenerosion unter Berücksichtigung des Zusammenhanges Erosion -Nährstoff- Abfluss im oberen Langete Einzugsgebiet- Physiogeographica, Ba.6, S. -242.

RoHrer, J. (1985): Quantitative Bestimmung der Bodenerosion unter Berücksichtigung des Zusammenhanges Erosion- Nährstoff-Abfluss im Oberen LangeteEinzugsgebiet.-Physiogeographica, BD 6, S.66 (242 S.)

Rorke, B. B. (Hrsg.) (1990): Soil Erosion. Experiments and Models - catena Suppl. $17,208 \mathrm{~S}$.

Ross, et al. (1999): Land use change: effects on soil carbon, nitrogen and phosphorus pools and fluxes in three adjacent ecosystems,- In Soil Biology \& Biochemistry, Bd. 31(1999), S. 803-813.

RupPERT, H. (1987): Bestimmung von Schwermetallgehalten im Boden sowie ihre Verhalten beeinflussende Bodeneigenschaften.- In: Beil. Zu GLA- Fachber., H.2, München.11S

S. Inthasothi, W. Jirasuktaveekul, W. Adirektrakam, S. Ratchadawong, A. BOONSANER (2003): Catchment Approach to Combating Soil Erosion in Thailand

S. M. VIRMANI (2003): Impacts of Land Degradation on Rainfed Maize Yield: The Northeast Thailand In: http://www.mekonginfo.org/mrc en/doclib.nsf/0/F7054648F6ED39E147256D6 50033D949/\$FILE/FULLTEXT.html

SPSS für Windows (2002): statistische Datenanalyse - Berlin ; Heidelberg : Springer XV, 722 S. : Ill., graph. Darst.

S. P. WANI (2003): Integrated Watershed Management for Sustaining Crop Productivity and Reducing Soil Erosion in Asia - In: http://www.mekonginfo.org/mrc en/doclib.nsf/0/3D0DC4683CFA695847256D 640035328C/\$FILE/FULLTEXT.html

SAIKH, H., VARADACHARI, C \& K. GHOSH (1998a): Changes in carbon, nitrogen and phosphorus levels due to deforestation and cultivation: A case study in Simlipal national Park, Indien. -In: Plant and Soil, Bd. 198 
SAIKH, H., VARADACHARI, C \& K. GHOSH (1998b): Effects of deforestation and cultivation on soil CEC and contents of exchangeable bases: A case study in Simlipal national Park, Indien. -In: Plant and Soil, Bd. 204

SANCHEZ, P. A. (1976): Properties and management of soils in the tropics. New York, John Wiley \& Sons, $618 \mathrm{p}$.

SANChez, P. A., BANDY, D. A, VillachicA, J. H. \& Nicholaides, J. J. (1982): Amazon basin soil: management for continuous crop production. Science 216. S821-827.

SAnchez, P. A., PALM, C. A. and SMYth, T. S. (1991): Phosphorus dynamic in shifting cultivation systems in the Amazon, in Tiesen, H., Lopez-Hernandez, D and Salcedo, I.H (eds.) Phosphorus cycles in terrestrial and aquatic ecosystems, pp. 142-160, Saskatoon, Canada.

Schaller, K. (1988): Praktikum zur Bodenkunde und Pflanzenernährung , Geisenheimer Berichte, Band 2.

SCHEFFER \& SCHACHTSCHABEL ( 1998): Lehrbuch der Bodenkunde- Ferdinand Enke Verlag Stuttgart 1998

SCHEFFER \& SCHACHTSCHABEL (1982): Lehrbuch der Bodenkunde, 11. Aufl. Stuttgart, Enke: $442 \mathrm{~S}$.

SCHEFFER \& SCHACHTSCHABEL (1992): Lehrbuch der Bodenkunde, Stuttgart

SCHERFFER \& SCHACHTSCHABEL (1999): Lehrbuchbodenkunde. Verlag Stuttgart.

SCHINNER, F. \& SONNLEITNER, R. (1996): Bodenökologie: Mikrobiologie und Bodenenzymatik: Grundlagen, Klima, Vegetation und Bodentyp, Springer Verlag

SCHINNER, F. \& SONNLEITNER, R. (1997): Bodenökologie: Mikrobiologie und Bodenenzymatik III: Pflanzenschutzmittel, Agrarhilfsstoffe und organische Umweltchemikalien. Springer - Verlag Berlin, Heidelberg, New York. Berlin, Heidelberg, New York. S 83-84

SCHNITZER, M. (1977): Recent finding on the characterization of humic substance extraed from soils from widely differing climatic zones. In Soil organic matter studies. Proceedings of a symposium by FAO. Braunschweig.

SchOLZ, H. (1984): Ist die Agrarproduktion der Tropen ökologisch benachteiligt? Geograpische Rundschau 36 (1984), S:360-366.

SchwertmanN, U. (1991): Aktuelle Probleme der Bodenerosion - Eine Einführung .- In: Bodennutzung und Bodenfruchtbarkeit Bd. 3, Verlag Paul Parey, Hamburg und Berlin, S.9-15

SchWertmann, U., Vogl, W., KaINZ, M (1987): Bodenerosion durch Wasser Vorhersage des Abtrags und Bewertung von Gegenmaßnahmen- Verlag Eugen Ulmer, Stuttgart.

Schwertmann, U., Vogl, W., KAINZ, M. (1990): Bodenerosion durch Wasser: Vorhersage des Abtrags und Bewertung von Gegenmaßnahmen- Ulmer, Stuttgart, 2. Auflage.

Sherchan, D. P., Chand, S. P., Thapa, Y. B., Tiwari, T. P., Gurung, G. B. (1990): Soil and nutrient losses in runoff on selected crop husbandry practices on hill slope soil of the Eastern Nepal. In: Proceedings, International symposium on water erosion, sedimentation and resource conservation. Dehra Dun, Central Soil and Water Conservation.

SIEM , N, T \& PHIEN, T.(1999): Upland soils in Vietnam- Degradation and rehabilitationAgriculture publishing House Hanoi

SinAJIN, J. S. (1987): Alternatives to shifting cultivation in Sabah, East Malaysia, Mardy/Malaysian Society of soil Science 
Sioli, H. (1984b): Forman and recent utilizations of Amazonian and their impact on the environment: Limnology and landscape ecology of a mighty tropical river and its basin. Dordrecht, Boston, Lancaster 1984 b S. 675-706.

SPENCER, J. E. (1966): Shifting cultivation in South-eastern Asia, University of California Press, Los Angeles, USA.

Steubing, L. \& Schwantes, H. O. (1981): Ökologische Botanik : Einführung in angewandte Botanik. Uni Taschenbuch. Heidelberg.

T. D. Toan, T. Phien, L. Nguyen, D. D. Thai \& N. V. Ga, (2003): Soil Erosion Management at the Watershed Level for Sustainable Agriculture and Forestry in Vietnam

TACIO, H. D. (1988): SALT: Sloping Agricultural Land Technology.- ILEIA- Newsletter, Vol.4 (No.1), 8-9.

TACIO, H. D. (1991): The SALT system Agro-forestry for sloping lands.- Agro-forestry Today, Vol.3 (No.1)12-13.

Tehrami, M. A. (1977): Einfluss von Bodenveränderungen durch Bodenerosion auf den Erntertrag untersuch auf vier Löss-Strandorten- Dissertation Giessen

THAI, P \& DAU, Q., A. (1997): Indigenous technical knowledge in Vietnam: Management of soil for agricultural development, S.101-120.

Thai, P \& NGuyen, T., S, (1997): Soil degradation and soil erosion control on sloping land in Vietnam, S.15-19 In Contour Newsletter of the Asia soil conservation network Vol. IX No. 1, 1997.

Thai, P, \& Nguyen, T., S (1998): Sustainable farming on sloping Lands in Vietnam, Hanoi 1998.

Thai, P. \& NGuyen, T, S. (1999): Upland soil in Vietnam - Degradation and Rehabilitation, Agriculture Publishing house, Hanoi, Vietnam.

Thai Phien AT AL (2000): Sustainable land management in Vietnam:assessment of hedgerow farming systems on sloping lands- Symposium no.38

ThORNES, J. B. (Hrsg.) (1990): Vegetation and erosion. - Chicheter, 518S.

Tiessen, H., Salcedo, I. H. \& SAmpaio, E. V. S. B. (1992): Nutrient and Soil organic matter dynamics under shifting cultivation in semi-arid North-Eastern Brazil. In: Agriculture Ecosystems \& Environment, Bd.39, S. 139-151.

Ton, T., C. \& LE, T, B. (1996): Su dung tot tai nguyen de bao ve moi truong, tap chi khoa hoc dat, Hanoi, 3/1996

Tran, D, V., (1996): Agriculture on Sloping lands challenges and Potentials: A selection of Studies done on the period of 1991-1996). Published on the Occasion of Celebrating 40 year of Hanoi Agricultural University No. I (HAU) and 17 year of Department of Agro-ecology and Environmental Science (DAES). Supported by: Vietnam National Universities Uplands Research and Development Network (VUN). Agricultural Publishing House, Hanoi. 470 S, S. 312-331

Tran, D. V. (1998): Soil erosion and Nutrient Balance in Swidden Fields of the Composite Swiddening Agro-ecosystem in the North-western Mountains of Vietnam .In Land degradation and Agricultural sustainability: Case Studies from Southeast and East Asia Edited by Aran Patanothai - Regional Secretariat- Khon kaen, Thailand.

Tran, V. T. (2000): Published and copyright by department of Geology and minerals of Vietnam. Hanoi 2000.

UNDP \& FAO (1996): Smallholder Reforestation In Central Vietnam, Hanoi.

VU TUYEN HOANG (2001): Bao ve moi truong va phat trien ben vung o Vietnam. 
WALter, H. (1964): Die Vegetation der Erde in ökophysiologischer Betrachtung. Bd. I. Jena, Gustav Fischer, 592 S.

Walter, H. (1979): Vegetation und Klimazonen. Die ökologische Gliederung der GeoBiosphäre, 4. Aufl. , Stuttgart, Ulmer, 342 S.

Webster, C. L. \& Wilson, P. N. (1996): Agriculture in the tropics, Verlag Longmans, London, 1996.

WeICHET, W. (1979): Einführung in die Klimaotologie-2. Aufl., Teubner, Stuttg.

WEICHET, W. (1981): Ackerland aus Tropenwald- eine verhängnisvolle Illusion. IN: Holz aktuell. Eine Zeitschr. Der Danzer- Unternehmen, H.3, S. 15-33.

WeISCHET, W. (1988): Einführung in die Allgemeine Klimatologie-physikalische und meteorologische Grundlage - Teubner, Stuttgart, 4. Auflage, S.264

WeltBAnK (1997): World Development Report 1997.

Whitmore, T. C. (1990): An introduction to tropical rainforests. Oxford University Press, Oxford, U. K.

WiSCHMEYER, W. (1959): A rainfall erosion index for a universal soil loss equation.- Soil Science America Proceedings., 23, S. 264-249.

WOLFRAM ACHTNICH (1986) : Landnutzung, Bodenfruchtbarkeit und Pflanzenproduktion in den Tropen und Subtropen, Heft 12- Göttingen beiträgt zur Land -und Fortwirtschaft in den Tropen und Subtropen.

WORLDBANK(2000):Statistical Appendix http://www.worldbank.org.vn/data_pub/reports/Bank1/rep34/statisti.pdf

WORLDTRAVELGUIDE.NET (2003): Columbus Publishing http://www.wtgonline.com/data/vnm/vnm500.asp

Young, A. (1976): Tropical Soil and Soil Survey. Cambridge, Cambridge University Press, $467 \mathrm{~S}$.

Young, A. (1989a): 10 Hypotheses for soil - agro-forestry research.- Agro-forestry Today, Vol.1(No.1),13-16

Young, A. (1989b): The Potential for Soil Conservation and Sustainable Land Use.Topic in Applied Ressource Management, Vol. 1, Selbstverlag Deutsches Institut für tropische und subtropische Landwirtschaft, Witzenhausen, 35-55.

YounG, A. (1990): Agro-forestry for Soil Conservation. - CAB International, Wallingford, U.K.

Zech, W., Senesi, N., Guggenberger, G., Haizer, K., Lermann, J., Miano, T. M. \& ACHROTH, G. (1997): Factors controlling humification and mineralization of soil organic matter in the tropics. Geoderma 79 (1997), S. 117-161. 


\section{ANHANG}

Karte 4:Anteil erodierter Fläche pro mapping unit

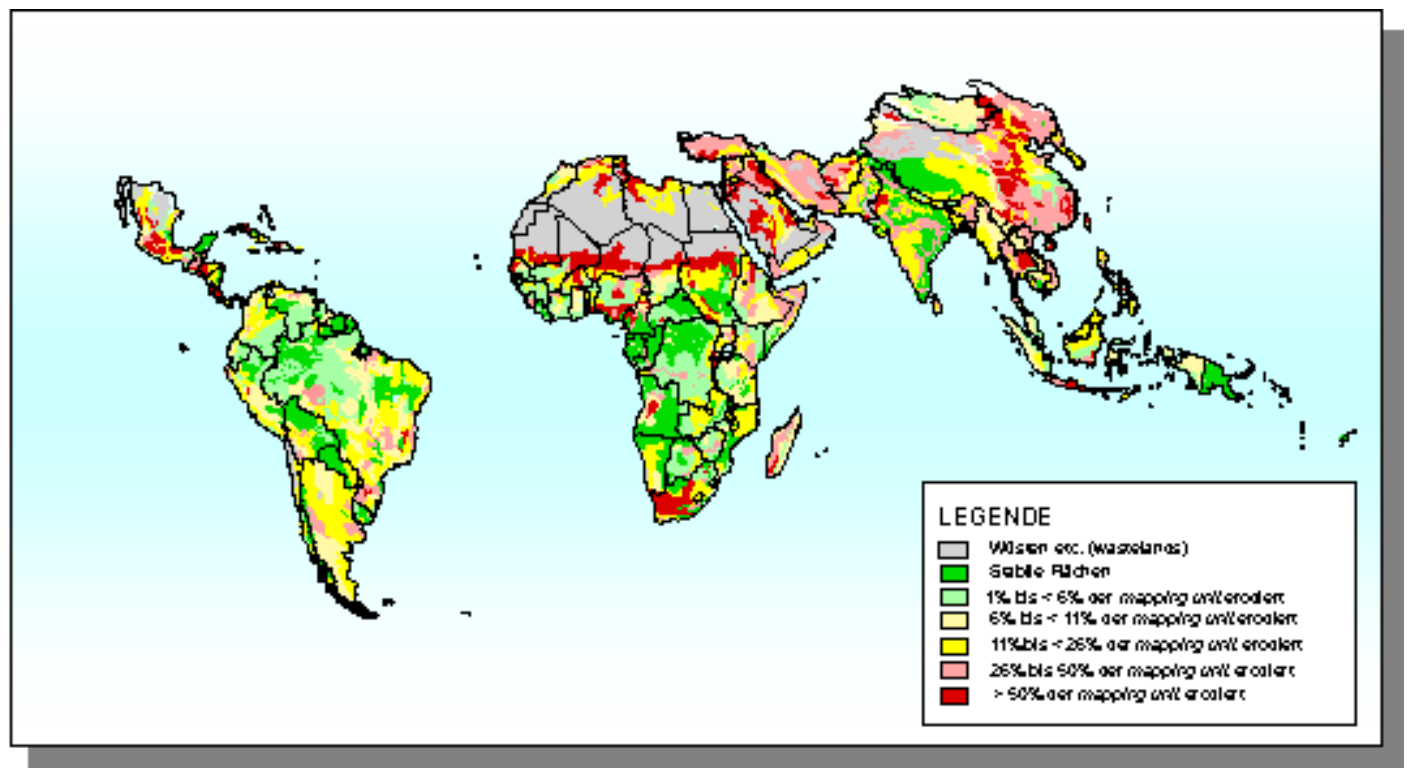

\section{Karte 5 : Haupterosionstyp pro mapping unit}

Quellen: GLASOD, 2000 aus http://edoc.hu-berlin.de/dissertationen/agrar/morgenrothsilvia/HTML/morgenroth-ch3.html

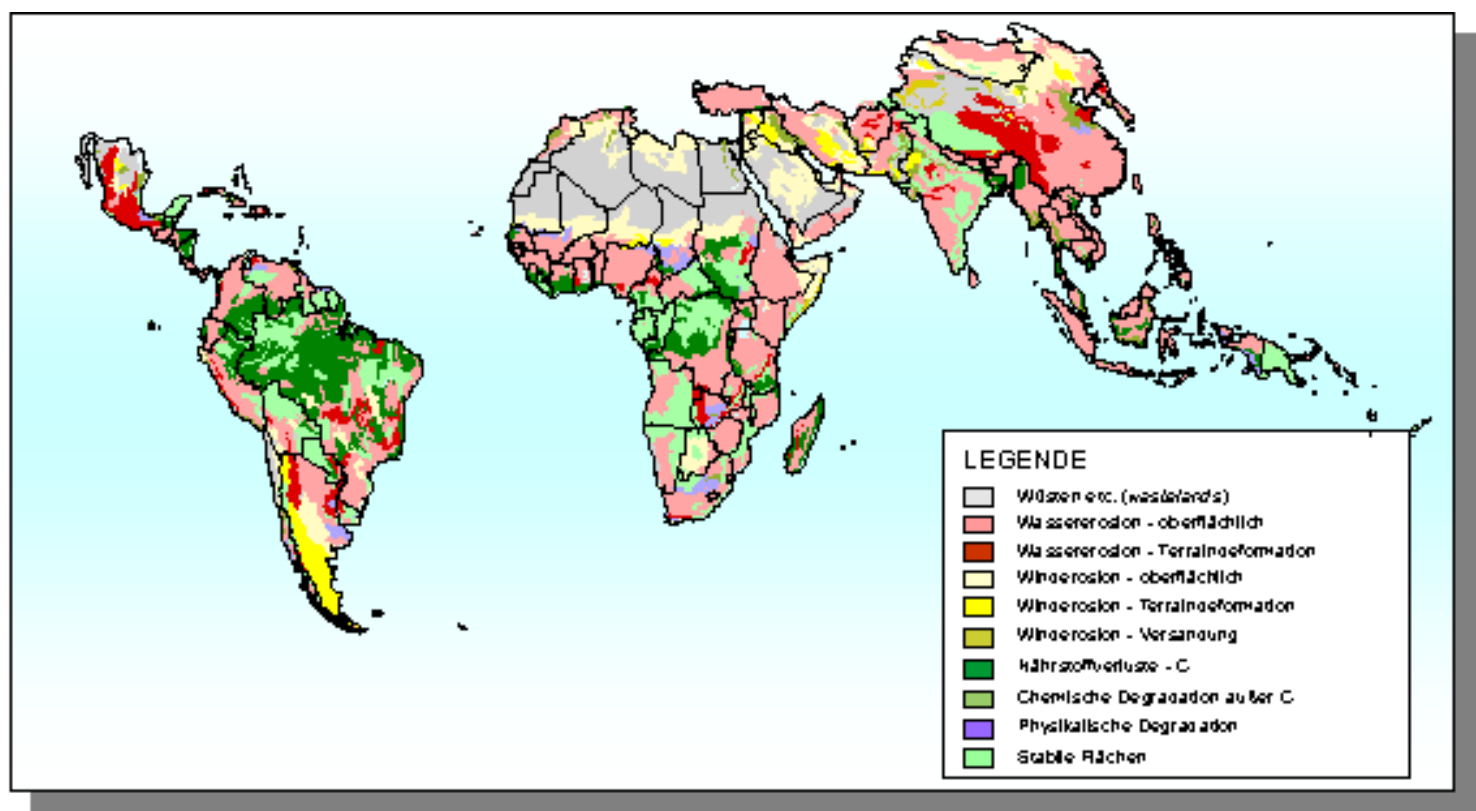


Anhang : Übersicht der am stärksten erodierten Länder nach Region

\begin{tabular}{|c|c|c|}
\hline \multirow{2}{*}{$\begin{array}{l}\text { Bodenerosion } \\
\text { insquesarnt }\end{array}$} & \multicolumn{2}{|c|}{ davon durch ${ }^{21}$} \\
\hline & $\begin{array}{l}\text { Wiaser Wuind } \\
\text { [W der erodierter }\end{array}$ & $\begin{array}{l}\text { Nährstoff- } \\
\text { verlus te } \\
\text { Flächel }\end{array}$ \\
\hline
\end{tabular}

\begin{tabular}{|c|c|}
\hline $\begin{array}{c}\text { Bodenerosion } \\
\text { mittel bis } \\
\text { extrem schwer } \\
{[\text { [ } \text { der Landestläche" }}\end{array}$ & $\begin{array}{l}\text { Nach } \\
\text { Schwereqrad } \\
\text { gewichteter } \\
\text { Frosionsindex }\end{array}$ \\
\hline
\end{tabular}

\begin{tabular}{|c|c|c|c|c|c|c|}
\hline \multicolumn{7}{|l|}{ AFRIKA } \\
\hline BURUNDI & 78 & 20 & 0 & 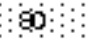 & 76 & 154 \\
\hline KAP VERDE & 75 & 00 & 0 & 0 & 75 & 225 \\
\hline TUNESIEN & 71 & 45 & 4 & 8 & 14 & $\infty$ \\
\hline RUANDA & 70 & 21 & 0 & ị: & 65 & 135 \\
\hline NIGER & 65 & 11 & $\Leftrightarrow$ & 0 & 35 & 102 \\
\hline MAURETANIEN & 63 & 0 & 0 & 0 & 26 & 95 \\
\hline BURKINAFASO & 42 & : & 15 & 0 & 37 & 107 \\
\hline MAL & 3 & 13 & 6 & 0 & 22 & 63 \\
\hline UBYEN & 8 & 5 & $\$ 5$ & 0 & 21 & 59 \\
\hline LESOTHO & 36 & 00 & 0 & 0 & 35 & 90 \\
\hline SÜDAFRIKA & 30 & $\phi$ & 1 & 0 & 30 & $\infty 6$ \\
\hline SUDAN & 30 & $3<$ & $4 z$ & : & 20 & 50 \\
\hline ALGER IEN & 30 & 22 & a & 7 & 12 & 42 \\
\hline TSCHAD & 30 & 8 & $\%$ & 3 & 9 & 39 \\
\hline MADAGASKAR & 28 & ar: & 1 & 19 & 28 & 72 \\
\hline NIGER IA & 27 & $g$ & 4 & 9 & 25 & 69 \\
\hline
\end{tabular}

\begin{tabular}{|llllllll|}
\hline SÜDAMERIKA & & & & & & \\
FALLAND INSELN & 28 & & 0 & 0 & 0 & 28 \\
\hline
\end{tabular}

\begin{tabular}{|c|c|c|c|c|c|c|}
\hline \multicolumn{7}{|c|}{ MITELAMERIKA } \\
\hline EL SALVADOR & 90 & 00 & 0 & 0 & 90 & 197 \\
\hline HAITI & 90 & 0 & 0 & 0 & $\infty$ & 269 \\
\hline COSTARICA & 62 & $\phi$ & 0 & 11 & 62 & 173 \\
\hline HONDURAS & 45 & 82 & 0 & 18 & 48 & 98 \\
\hline GUATEMALA & 41 & $\Leftrightarrow$ & 0 & 17 & 41 & 85 \\
\hline PANAMA & $\infty$ & $g \mathrm{~s}$ & 0 & 7 & 36 & $\infty$ \\
\hline NICARAGUA & 29 & 54 & 0 & क & 27 & 57 \\
\hline
\end{tabular}

\begin{tabular}{|c|c|c|c|c|c|c|}
\hline $\begin{array}{l}\text { SÜDOSTASIEN } \\
\text { TAIWIAN }\end{array}$ & 43 & 100 & 0 & 0 & 0 & 43 \\
\hline THALAAND & 37 & $\phi 0$ & 0 & 10 & 37 & 102 \\
\hline SRI LANKA & 33 & 60 & 0 & $9 \mathrm{a}$ & 19 & 65 \\
\hline CHINA & 3 & $7 z$ & 28 & 0 & 19 & 54 \\
\hline VIETNAM & 27 & of & 0 & 19 & 25 & 71 \\
\hline KOREA (NORD) & 27 & 00 & 0 & 0 & 0 & 27 \\
\hline
\end{tabular}

\begin{tabular}{|c|c|c|c|c|c|c|}
\hline \multicolumn{7}{|c|}{ SÜDWESTASIEN } \\
\hline SAUDDFARABIEN & 53 & 0 & 00 & 0 & 12 & 65 \\
\hline JORDANIEN & 39 & 10 & 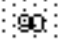 & 0 & 17 & 56 \\
\hline TURKEi & 39 & 4 & 0 & 6 & 39 & 95 \\
\hline JEMEN & 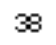 & 4 & o & 3 & 12 & 50 \\
\hline SYRIEN & $\infty$ & 17 & 4 & 6 & 27 & 62 \\
\hline OMAN & $\$ 5$ & 4 & ; & 0 & 17 & 52 \\
\hline IRAN & 34 & 5 & 4 & 0 & 15 & 48 \\
\hline AFGHANISTAN & 32 & 8 & 13 & 0 & 8 & 3 \\
\hline
\end{tabular}

) Landesfläche bezeichnet hier die Referenzfläche, d.h. die Landesfläche abzüglich der wastelands 2) $\mathrm{Die}$ in einem Land dominierende(n) Erosionsart(en) ist (sind) grau unterlegt Nährstoffverluste bezeichnet hier den Verlust von Nährstoffen und organischer Substanz (C) 3) Schweregrade 2 bis 4 gemäß GLASOD-Definition (WEC2_4) 4) Die erodierten Flächen eines Landes werden je nach Schweregrad gewichtet (WEC_s) 
Legende verändert nach G. Haase, 1964, In: Barsch.H, Billwitz,K \& Bork H-R. (2000), S. $176 \& 177$

1. Organische Bodenkomponente

a) unzersetzte Streu

Goosono lose Streu

b) Auflagehumus und Humus im Mineralboden

\section{Mull}

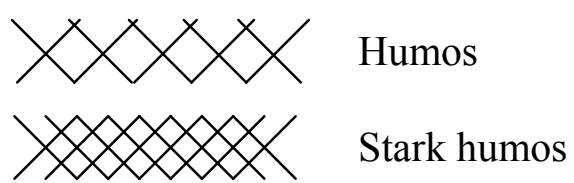

2. Anorganische Bodenkomponente

$$
\begin{aligned}
& 0 \quad 00 \quad \text { Steine } \\
& 0_{0} \circ 0_{0}^{\circ} \text { Grus, Kies } \\
& \therefore \therefore: \therefore \quad \text { Sand } \quad \text { 三:三:三:ニ Schluff }=\text { Ton }
\end{aligned}
$$

3. Bodendynamik

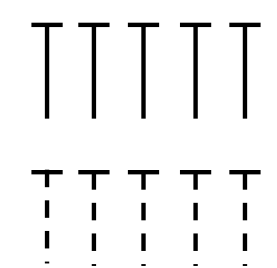

verwitterungsresistente Minerale (Fe-, Al-, Mn-Oxide) und mit Sesquioxiden durch Umlagerung angereicherter B-Horizont

Lessivierung, Ac oder Al, Tonverarmungshorizont

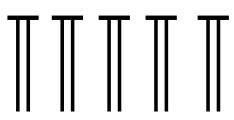

Lessivierung, Bt, Tonanreicherungshorizont

$\because \because \therefore$

Pseudovergleyung, go, Rostfleckung, vor allem infolgeOxydation des Eisens

Pseudovergleyung; go, einzelne Rostflecken

$\sim \because \because \therefore \sim \quad$ Vergleyung; Gr+ o, Reduktions-Oxydationhorizont

4. Sonstige Zeichen

Konkretionen, vor allem von Eisen und Mangan (bei Pseudogley) 
Durchschnittliche Klimadaten des Distrikts Ba Be in den Jahren 1996-2000

\begin{tabular}{lrrrrrrrrrrrr}
\hline Monate & Jan. & Feb. & Mär. Apr. & Mai & Jun & Jul & Aug & Sep & Oct & Nov & Dec \\
\hline Sonnenstunden & 74,8 & 46,0 & 50,0 & 114,0 & 148,8 & 134,3 & 140,3 & 174,5 & 152,3 & 136,5 & 121,5 & 105,8 \\
\hline Luftfeuchtigkeit \% & 81,8 & 80,0 & 82,3 & 81,0 & 82,8 & 84,8 & 86,3 & 85,5 & 83,8 & 85,5 & 82,8 & 80,3 \\
\hline Temp. Max. $\left({ }^{\circ} \mathrm{C}\right)$ & 18 & 18,2 & 22,8 & 26,5 & 28,6 & 31,9 & 33,7 & 33,1 & 30,4 & 26,0 & 22,8 & 18,4 \\
\hline Temp. Mittel $\left({ }^{\circ} \mathrm{C}\right)$ & 16,2 & 16,8 & 20,1 & 24,3 & 26,0 & 27,8 & 27,8 & 27,8 & 25,8 & 23,6 & 19,8 & 16,6 \\
\hline Temp. Min $\left({ }^{\circ} \mathrm{C}\right)$ & 9,5 & 12,3 & 14,2 & 21,0 & 23,3 & 24,6 & 24,4 & 25,1 & 23,6 & 20,2 & 15,8 & 12,9 \\
\hline Niederschlag $(\mathrm{mm})$ & 29,0 & 12,4 & 66,9 & 155,9 & 205,7 & 244,4 & 276,7 & 244,8 & 101,8 & 117,9 & 21,6 & 14,1 \\
\hline Evaporation $(\mathrm{mm})$ & 57,4 & 60,5 & 66,1 & 71,4 & 73,4 & 65,3 & 59,8 & 67,2 & 70,3 & 63,7 & 61,7 & 61,2 \\
\hline
\end{tabular}

\section{Niederschlag des Distrikts Babe von 1996-2000 und Mittelwert über 20 Jahre}

\begin{tabular}{lrrrrrr}
\hline Monate & 1996 & 1997 & 1998 & 1999 & 2000 & Mittelwert 20 Jahre \\
\hline Jan. & 69,30 & 63,30 & 1,50 & 1,80 & 8,90 & 45,60 \\
\hline Feb. & 6,70 & 3,60 & 10,00 & 3,50 & 38,10 & 10,20 \\
\hline März. & 16,60 & 142,20 & 93,30 & 24,00 & 58,00 & 95,40 \\
\hline April & 230,50 & 151,40 & 110,40 & 198,50 & 88,50 & 200,20 \\
\hline Mai & 326,70 & 106,60 & 82,50 & 191,70 & 320,90 & 158,40 \\
\hline Jun & 218,30 & 176,50 & 339,80 & 169,80 & 117,50 & 228,10 \\
\hline Jul & 211,20 & 275,70 & 262,30 & 187,20 & 297,10 & 269,30 \\
\hline Aug & 325,60 & 259,70 & 127,10 & 411,40 & 100,20 & 254,20 \\
\hline Sep & 72,60 & 89,00 & 81,80 & 87,40 & 178,30 & 123,20 \\
\hline Okt & 97,20 & 86,00 & 16,10 & 107,80 & 282,40 & 98,70 \\
\hline Nov & 38,20 & 3,20 & 22,30 & 42,40 & 2,00 & 12,50 \\
\hline Dez & 25,90 & 11,80 & 6,20 & 22,70 & 3,80 & 22,40 \\
\hline Gesamt & 1638,80 & 1369,00 & 1153,30 & 1448,20 & 1495,70 & 1518,20 \\
\hline
\end{tabular}


Die Veränderung der Bodenfruchtbarkeit: Vor dem Anbau und nach der Ernte im 1 Jahr nach Brandrodung

\begin{tabular}{|c|c|c|c|c|c|c|c|c|c|c|c|c|c|c|c|c|}
\hline Parzellen & $\begin{array}{l}\text { Tief } \\
\text { (cm) }\end{array}$ & $\begin{array}{l}\text { pH } \\
\mathbf{H}_{2} \mathbf{0}\end{array}$ & $\begin{array}{l}\text { pH } \\
\text { KCL }\end{array}$ & $\begin{array}{l}\mathrm{C} \\
(\%)\end{array}$ & \begin{tabular}{|l|}
$\mathbf{N}$ \\
$(\%)$
\end{tabular} & $\mathbf{C} / \mathbf{N}$ & $\begin{array}{l}\text { C } \\
\text { org }\end{array}$ & $\begin{array}{l}\text { KAK } \\
\text { Al } \\
\text { cmol/kg } \\
\end{array}$ & \begin{tabular}{|l|}
$\mathrm{KAK}$ \\
$\mathrm{Ca}$ \\
$\mathrm{cmol} / \mathrm{kg}$ \\
\end{tabular} & $\begin{array}{l}\text { KAK } \\
\mathrm{Fe} \\
\mathrm{cmol} / \mathrm{kg} \\
\end{array}$ & \begin{tabular}{|l|}
$\mathrm{KAK}$ \\
$\mathrm{K}$ \\
$\mathrm{cmol} / \mathrm{kg}$ \\
\end{tabular} & \begin{tabular}{|l|}
$\mathrm{KAK}$ \\
$\mathrm{Mg}$ \\
$\mathrm{cmol} / \mathrm{kg}$ \\
\end{tabular} & \begin{tabular}{|l|}
$\mathrm{KAK}$ \\
$\mathrm{Mn}$ \\
$\mathrm{cmol} / \mathrm{kg}$ \\
\end{tabular} & \begin{tabular}{|l|}
$\mathrm{KAK}$ \\
$\mathrm{Na}$ \\
$\mathrm{cmol} / \mathrm{kg}$ \\
\end{tabular} & $\begin{array}{l}\text { KAK } \\
\text { eff }\end{array}$ & \begin{tabular}{|l|} 
V- \\
wert \\
$\%$ \\
\end{tabular} \\
\hline \multicolumn{17}{|c|}{ Vor dem Anbau } \\
\hline V1 & $0-30$ & 6,93 & 6,24 & 2,92 & 0,25 & 11,89 & 5,0 & 0,0 & 8,1 & 0,0 & 1,5 & 3,9 & 0,0 & 0,0 & 13,5 & 99,7 \\
\hline V2 & $0-30$ & 7,00 & 6,38 & 2,30 & 0,19 & 12,01 & 4,0 & 0,0 & 6,8 & 0,0 & 2,3 & 3,2 & 0,0 & 0,0 & 12,3 & 99,3 \\
\hline V3 & $0-30$ & 5,75 & 4,73 & 2,36 & 0,20 & 12,12 & 4,1 & 0,2 & 3,8 & 0,0 & 0,7 & 1,7 & 0,0 & 0,0 & 6,3 & 97,2 \\
\hline \multicolumn{17}{|c|}{ Nach der Ernte } \\
\hline N1 & $0-30$ & 5,81 & 4,56 & 1,66 & 0,14 & 11,79 & 2,8 & 0,4 & 2,9 & 0,0 & 0,6 & 1,8 & 0,0 & 0,0 & 5,7 & 92,1 \\
\hline $\mathrm{N} 2$ & $0-30$ & 5,52 & 4,46 & 1,99 & 0,16 & 12,48 & 3,4 & 0,7 & 3,4 & 0,0 & 0,6 & 1,6 & 0,0 & 0,0 & 6,4 & 88,3 \\
\hline N3 & $0-30$ & 5,57 & 4,45 & 2,04 & 0,15 & 13,92 & 3,5 & 0,5 & 2,8 & 0,0 & 0,6 & 1,4 & 0,0 & 0,0 & 5,3 & 89,6 \\
\hline \multicolumn{17}{|c|}{ Vor dem Anbau } \\
\hline V1 & $31-60$ & 5,58 & 4,55 & 2,174 & 0,18 & 11,85 & 3,7 & 0,6 & 2,1 & 0,0 & 0,5 & 1,5 & 0,0 & 0,0 & 4,9 & 86,3 \\
\hline $\mathrm{V} 2$ & $31-60$ & 5,37 & 4,44 & 1,579 & 0,13 & 12,05 & 2,7 & 0,8 & 2,0 & 0,0 & 0,5 & 1,1 & 0,0 & 0,0 & 4,4 & 80,5 \\
\hline V3 & $31-60$ & 5,52 & 4,56 & 1,620 & 0,14 & 11,53 & 2,8 & 0,3 & 2,9 & 0,0 & 0,5 & 1,4 & 0,0 & 0,0 & 5,1 & 93,2 \\
\hline \multicolumn{17}{|c|}{ Nach der Ernte } \\
\hline N1 & $31-60$ & 5,69 & 4,52 & 1,76 & 0,16 & 11,12 & 3,0 & 0,4 & 1,7 & 0,0 & 0,3 & 0,6 & 0,1 & 0,0 & 5,1 & 50,6 \\
\hline $\mathrm{N} 2$ & $31-60$ & 5,33 & 4,21 & 1,22 & 0,09 & 13,32 & 2,1 & 1,7 & 1,1 & 0,0 & 0,3 & 0,7 & 0,0 & 0,0 & 3,9 & 55,2 \\
\hline N3 & $31-60$ & 5,40 & 4,21 & 0,99 & 0,08 & 11,75 & 1,7 & 1,3 & 1,0 & 0,0 & 0,3 & 0,6 & 0,0 & 0,0 & 3,2 & 58,2 \\
\hline
\end{tabular}


Die Veränderung der Bodenfruchtbarkeit: Vor dem Anbau und nach der Ernte im 4.Jahr nach Brandrodung (Ende der Fruchtfolge)

\begin{tabular}{|c|c|c|c|c|c|c|c|c|c|c|c|c|c|c|c|c|}
\hline Parzellen & $\begin{array}{l}\text { Tief } \\
(\mathrm{cm})\end{array}$ & $\begin{array}{l}\mathrm{pH} \\
\mathrm{H}_{2} \mathrm{O}\end{array}$ & \begin{tabular}{|l|} 
pH \\
KCL
\end{tabular} & $\begin{array}{l}\mathbf{C} \\
(\%)\end{array}$ & $\begin{array}{l}\mathbf{N} \\
(\%)\end{array}$ & $\mathbf{C} / \mathbf{N}$ & \begin{tabular}{|l|} 
C \\
org
\end{tabular} & \begin{tabular}{|l} 
KAK \\
Al \\
cmol/kg
\end{tabular} & \begin{tabular}{|l|}
$\mathrm{KAK}$ \\
$\mathrm{Ca}$ \\
$\mathrm{cmol} / \mathrm{kg}$
\end{tabular} & $\begin{array}{l}\text { KAK } \\
\mathrm{Fe} \\
\mathrm{cmol} / \mathrm{kg}\end{array}$ & \begin{tabular}{|l|}
$\mathrm{KAK}$ \\
$\mathrm{K}$ \\
$\mathrm{cmol} / \mathrm{kg}$ \\
\end{tabular} & \begin{tabular}{|l|}
$\mathrm{KAK}$ \\
$\mathrm{Mg}$ \\
$\mathrm{cmol} / \mathrm{kg}$
\end{tabular} & \begin{tabular}{|l|}
$\mathrm{KAK}$ \\
$\mathrm{Mn}$ \\
$\mathrm{cmol} / \mathrm{kg}$
\end{tabular} & \begin{tabular}{|l|}
$\mathrm{KAK}$ \\
$\mathrm{Na}$ \\
$\mathrm{cmol} / \mathrm{kg}$
\end{tabular} & $\begin{array}{l}\text { KAK } \\
\text { eff }\end{array}$ & \begin{tabular}{|l|} 
V- \\
wert \\
$\%$ \\
\end{tabular} \\
\hline \multicolumn{17}{|c|}{ Vor dem Anbau } \\
\hline V1 & $0-30$ & 5,40 & 4,40 & 1,34 & 0,11 & 12,72 & 2,3 & 0,4 & 2,8 & 0,0 & 0,4 & 1,0 & 0,0 & 0,0 & 4,5 & 91,5 \\
\hline V2 & $0-30$ & 5,64 & 4,43 & 1,23 & 0,11 & 11,54 & 2,1 & 0,4 & 3,7 & 0,0 & 0,7 & 1,1 & 0,1 & 0,0 & 5,9 & 92,2 \\
\hline V3 & $0-30$ & 6,00 & 4,87 & 1,67 & 0,15 & 11,43 & 2,9 & 0,1 & 3,3 & 0,0 & 0,7 & 1,0 & 0,0 & 0,0 & 8,7 & 57,5 \\
\hline \multicolumn{17}{|c|}{ Nach der Ernte } \\
\hline N1 & $0-30$ & 5,86 & 4,61 & 0,87 & 0,08 & 11,53 & 1,5 & 0,3 & 2,4 & 0,0 & 0,7 & 1,1 & 0,0 & 0,0 & 5,5 & 76,4 \\
\hline $\mathrm{N} 2$ & $0-30$ & 6,10 & 4,72 & 1,00 & 0,11 & 9,09 & 1,7 & 0,1 & 2,9 & 0,0 & 0,7 & 1,2 & 0,0 & 0,0 & 6,1 & 78,7 \\
\hline N3 & $0-30$ & 5,62 & 4,33 & 1,41 & 0,12 & 12,53 & 2,4 & 0,4 & 2,1 & 0,0 & 0,5 & 1,2 & 0,0 & 0,0 & 6,2 & 61,3 \\
\hline \multicolumn{17}{|c|}{ Vor dem Anbau } \\
\hline V1 & $31-60$ & 5,72 & 4,49 & 0,95 & 0,09 & 11,20 & 1,6 & 0,3 & 1,6 & 0,0 & 0,6 & 1,0 & 0,0 & 0,0 & 4,6 & 69,6 \\
\hline $\mathrm{V} 2$ & $31-60$ & 5,58 & 4,33 & 0,92 & 0,08 & 11,27 & 1,5 & 0,7 & 2,8 & 0,0 & 0,5 & 0,9 & 0,0 & 0,0 & 4,9 & 84,9 \\
\hline V3 & $31-60$ & 6,15 & 4,96 & 1,36 & 0,11 & 12,22 & 2,3 & 0,1 & 2,3 & 0,0 & 0,6 & 1,5 & 0,0 & 0,0 & 7,4 & 60,0 \\
\hline \multicolumn{17}{|c|}{ Nach der Ernte } \\
\hline N1 & $31-60$ & 6,00 & 4,57 & 0,72 & 0,06 & 11,29 & 1,2 & 0,2 & 1,4 & 0,0 & 0,6 & 1,0 & 0,0 & 0,0 & 5,3 & 56,6 \\
\hline N2 & $31-60$ & 5,88 & 4,52 & 1,07 & 0,09 & 12,44 & 1,8 & 0,2 & 2,4 & 0,0 & 0,7 & 1,0 & 0,0 & 0,0 & 6,5 & 63,1 \\
\hline N3 & $31-60$ & 5,50 & 4,30 & 1,22 & 0,09 & 12,91 & 2,1 & 0,5 & 2,6 & 0,0 & 0,5 & 1,3 & 0,0 & 0,0 & 6,9 & 63,8 \\
\hline
\end{tabular}


Die Veränderung der Bodenfruchtbarkeit: April und Oktober auf 4 Jahre alter Brache

\begin{tabular}{|c|c|c|c|c|c|c|c|c|c|c|c|c|c|c|c|c|}
\hline Parzellen & $\begin{array}{l}\text { Tief } \\
\text { (cm) }\end{array}$ & $\begin{array}{l}\mathrm{pH} \\
\mathrm{H}_{2} \mathrm{O}\end{array}$ & \begin{tabular}{|l} 
pH \\
KCL
\end{tabular} & \begin{tabular}{|l}
$\mathrm{C}$ \\
$(\%)$
\end{tabular} & \begin{tabular}{|l|}
$\mathbf{N}$ \\
$(\%)$
\end{tabular} & $\mathbf{C} / \mathbf{N}$ & \begin{tabular}{|l|} 
C \\
org
\end{tabular} & $\begin{array}{l}\text { KAK } \\
\text { Al } \\
\text { cmol/kg }\end{array}$ & \begin{tabular}{|l|} 
KAK \\
$\mathrm{Ca}$ \\
$\mathrm{cmol} / \mathrm{kg}$
\end{tabular} & $\begin{array}{l}\mathrm{KAK} \\
\mathrm{Fe} \\
\mathrm{cmol} / \mathrm{kg}\end{array}$ & \begin{tabular}{|l} 
KAK \\
K \\
$\mathbf{c m o l} / \mathbf{k g}$
\end{tabular} & $\begin{array}{l}\mathrm{KAK} \\
\mathrm{Mg} \\
\mathrm{cmol} / \mathrm{kg}\end{array}$ & \begin{tabular}{|l|} 
KAK \\
Mn \\
cmol/kg
\end{tabular} & \begin{tabular}{|l|}
$\mathrm{KAK}$ \\
$\mathrm{Na}$ \\
$\mathrm{cmol} / \mathrm{kg}$
\end{tabular} & $\begin{array}{l}\text { KAK } \\
\text { eff }\end{array}$ & \begin{tabular}{|l|} 
V- \\
wert \\
$\%$
\end{tabular} \\
\hline \multicolumn{17}{|l|}{ April } \\
\hline V1 & $0-30$ & 5,84 & 4,82 & 2,96 & 0,23 & 13,12 & 5,1 & 0,1 & 4,2 & 0,0 & 1,0 & 2,6 & 0,1 & 0,0 & 8,6 & 90,6 \\
\hline V2 & $0-30$ & 5,82 & 4,90 & 2,60 & 0,20 & 13,19 & 4,5 & 0,1 & 3,9 & 0,0 & 1,1 & 2,3 & 0,0 & 0,0 & 8,5 & 85,9 \\
\hline V3 & $0-30$ & 5,60 & 4,56 & 2,73 & 0,22 & 12,18 & 4,6 & 0,3 & 4,1 & 0,0 & 0,7 & 1,9 & 0,0 & 0,0 & 6,9 & 96,0 \\
\hline \multicolumn{17}{|l|}{ Oktober } \\
\hline N1 & $0-30$ & 5,90 & 4,63 & 2,12 & 0,18 & 11,78 & 3,6 & 0,1 & 4,0 & 0,0 & 0,8 & 2,2 & 0,0 & 0,0 & 7,1 & 98,6 \\
\hline $\mathrm{N} 2$ & $0-30$ & 5,81 & 4,75 & 2,06 & 0,17 & 12,53 & 3,5 & 0,1 & 3,0 & 0,0 & 0,9 & 2,0 & 0,0 & 0,0 & 6,9 & 85,5 \\
\hline N3 & $0-30$ & 5,76 & 4,70 & 2,21 & 0,18 & 12,39 & 3,8 & 0,1 & 3,7 & 0,0 & 0,6 & 1,7 & 0,0 & 0,0 & 6,2 & 97,4 \\
\hline \multicolumn{17}{|l|}{ April } \\
\hline V1 & $31-60$ & 5,42 & 4,29 & 1,71 & 0,13 & 13,16 & 3,0 & 0,7 & 2,0 & 0,0 & 0,5 & 1,3 & 0,0 & 0,0 & 4,6 & 82,8 \\
\hline V2 & $31-60$ & 5,50 & 4,45 & 1,35 & 0,10 & 13,32 & 2,3 & 0,3 & 3,0 & 0,0 & 0,9 & 1,6 & 0,0 & 0,0 & 6,0 & 75,0 \\
\hline V3 & $31-60$ & 4,92 & 4,14 & 1,15 & 0,09 & 12,72 & 2,0 & 0,9 & 1,5 & 0,0 & 0,1 & 1,0 & 0,0 & 0,0 & 3,5 & 74,1 \\
\hline \multicolumn{17}{|l|}{ Oktober } \\
\hline $\mathrm{N} 1$ & $31-60$ & 5,55 & 4,42 & 2,08 & 0,15 & 13,86 & 3,5 & 0,3 & 2,9 & 0,0 & 0,5 & 1,8 & 0,0 & 0,0 & 6,5 & 80,1 \\
\hline N2 & $31-60$ & 5,70 & 4,46 & 1,17 & 0,10 & 11,70 & 2,1 & 0,5 & 1,5 & 0,0 & 0,4 & 0,9 & 0,0 & 0,0 & 3,4 & 84,8 \\
\hline N3 & $31-60$ & 5,78 & 4,55 & 1,50 & 0,14 & 10,85 & 2,6 & 0,3 & 2,4 & 0,0 & 1,0 & 1,6 & 0,0 & 0,1 & 6,4 & 79,7 \\
\hline
\end{tabular}


Die Veränderung der Bodenfruchtbarkeit: im April und im Oktober im Agroforstsystem mit Mulch

\begin{tabular}{|c|c|c|c|c|c|c|c|c|c|c|c|c|c|c|c|c|}
\hline Parzellen & $\begin{array}{l}\text { Tief } \\
(\mathrm{cm})\end{array}$ & \begin{tabular}{|l|}
$\mathbf{p H}$ \\
$\mathbf{H}_{2} \mathbf{O}$
\end{tabular} & \begin{tabular}{|l} 
pH \\
KCL
\end{tabular} & \begin{tabular}{|l|}
$C$ \\
$(\%)$
\end{tabular} & \begin{tabular}{|l|}
$N$ \\
$(\%)$
\end{tabular} & $\mathbf{C} / \mathbf{N}$ & \begin{tabular}{|l|} 
C \\
org
\end{tabular} & \begin{tabular}{|l} 
KAK \\
Al \\
cmol/kg
\end{tabular} & $\begin{array}{l}\mathrm{KAK} \\
\mathrm{Ca} \\
\mathrm{cmol} / \mathrm{kg}\end{array}$ & $\begin{array}{l}\text { KAK } \\
\mathrm{Fe} \\
\mathrm{cmol} / \mathrm{kg}\end{array}$ & \begin{tabular}{|l|}
$\mathrm{KAK}$ \\
$\mathrm{K}$ \\
$\mathrm{cmol} / \mathrm{kg}$ \\
\end{tabular} & \begin{tabular}{|l|}
$\mathrm{KAK}$ \\
$\mathrm{Mg}$ \\
$\mathrm{cmol} / \mathrm{kg}$
\end{tabular} & \begin{tabular}{|l|}
$\mathrm{KAK}$ \\
$\mathrm{Mn}$ \\
$\mathrm{cmol} / \mathrm{kg}$
\end{tabular} & \begin{tabular}{|l|}
$\mathrm{KAK}$ \\
$\mathrm{Na}$ \\
$\mathrm{cmol} / \mathrm{kg}$
\end{tabular} & $\begin{array}{l}\text { KAK } \\
\text { eff }\end{array}$ & \begin{tabular}{|l|} 
V- \\
wert \\
$\%$ \\
\end{tabular} \\
\hline \multicolumn{17}{|l|}{ April } \\
\hline V1 & $0-30$ & 4,90 & 4,28 & 2,06 & 0,15 & 14,19 & 3,5 & 1,2 & 0,8 & 0,0 & 0,4 & 0,5 & 0,1 & 0,0 & 3,0 & 56,9 \\
\hline V2 & $0-30$ & 5,01 & 4,30 & 1,71 & 0,12 & 14,74 & 3,0 & 1,0 & 0,8 & 0,0 & 0,3 & 0,6 & 0,0 & 0,0 & 2,7 & 61,9 \\
\hline V3 & $0-30$ & 4,94 & 4,34 & 1,97 & 0,16 & 12,16 & 3,4 & 0,8 & 1,2 & 0,0 & 0,4 & 0,7 & 0,1 & 0,0 & 3,1 & 71,0 \\
\hline \multicolumn{17}{|l|}{ Oktober } \\
\hline N1 & $0-30$ & 4,6 & 4,10 & 1,50 & 0,10 & 15,50 & 2,6 & 2,0 & 0,3 & 0,0 & 0,1 & 0,2 & 0,0 & 0,0 & 2,6 & 23,1 \\
\hline $\mathrm{N} 2$ & $0-30$ & 6,10 & 5,58 & 2,87 & 0,22 & 13,20 & 4,9 & 0,0 & 11,5 & 0,0 & 0,6 & 1,3 & 0,0 & 0,0 & 13,5 & 99,5 \\
\hline N3 & $0-30$ & 6,79 & 6,10 & 3,84 & 0,24 & 15,80 & 6,6 & 0,0 & 15,5 & 0,0 & 0,8 & 1,7 & 0,0 & 0,0 & 18,2 & 99,6 \\
\hline \multicolumn{17}{|l|}{ April } \\
\hline V1 & $31-60$ & 5,00 & 4,25 & 1,58 & 0,12 & 13,32 & 2,7 & 1,2 & 0,6 & 0,0 & 0,1 & 0,3 & 0,0 & 0,0 & 2,3 & 43,4 \\
\hline V2 & $31-60$ & 5,00 & 4,26 & 1,31 & 0,09 & 15,23 & 2,3 & 1,1 & 0,5 & 0,0 & 0,1 & 0,4 & 0,0 & 0,0 & 2,1 & 46,7 \\
\hline V3 & $31-60$ & 4,80 & 4,20 & 1,52 & 0,12 & 13,07 & 2,6 & 1,4 & 1,0 & 0,0 & 0,2 & 0,6 & 0,1 & 0,0 & 3,2 & 32,4 \\
\hline \multicolumn{17}{|l|}{ Oktober } \\
\hline \begin{tabular}{|l} 
N1 \\
\end{tabular} & $31-60$ & 4,59 & 4,10 & 1,15 & 0,09 & 13,15 & 2,0 & 1,9 & 0,3 & 0,0 & 0,1 & 0,2 & 0,0 & 0,0 & 2,6 & 22,9 \\
\hline N2 & $31-60$ & 5,10 & 4,29 & 1,67 & 0,13 & 13,30 & 2,8 & 1,2 & 0,9 & 0,0 & 0,2 & 0,6 & 0,0 & 0,0 & 3,0 & 56,0 \\
\hline N3 & $31-60$ & 4,87 & 4,30 & 1,72 & 0,13 & 13,74 & 3,0 & 1,1 & 0,8 & 0,0 & 0,2 & 0,5 & 0,0 & 0,0 & 2,7 & 56,5 \\
\hline
\end{tabular}




\begin{tabular}{|c|c|c|c|c|c|c|c|c|c|c|c|c|c|c|c|c|}
\hline Parzellen & \begin{tabular}{|l|} 
Tief \\
(cm)
\end{tabular} & $\begin{array}{l}\mathrm{pH} \\
\mathrm{H}_{2} \mathrm{O}\end{array}$ & $\begin{array}{l}\text { pH } \\
\text { KCL }\end{array}$ & \begin{tabular}{|l|}
$C$ \\
$(\%)$
\end{tabular} & \begin{tabular}{|l|}
$N$ \\
$(\%)$
\end{tabular} & $\mathbf{C} / \mathbf{N}$ & \begin{tabular}{|l|}
$\mathrm{C}$ \\
org
\end{tabular} & \begin{tabular}{|l} 
KAK \\
Al \\
cmol/kg
\end{tabular} & $\begin{array}{l}\mathrm{KAK} \\
\mathrm{Ca} \\
\mathrm{cmol} / \mathrm{kg}\end{array}$ & \begin{tabular}{|l|}
$\mathrm{KAK}$ \\
$\mathrm{Fe}$ \\
$\mathrm{cmol} / \mathrm{kg}$
\end{tabular} & \begin{tabular}{|l|} 
KAK \\
K \\
cmol/kg
\end{tabular} & \begin{tabular}{|l|}
$\mathrm{KAK}$ \\
$\mathrm{Mg}$ \\
$\mathrm{cmol} / \mathrm{kg}$
\end{tabular} & \begin{tabular}{|l|} 
KAK \\
Mn \\
cmol/kg
\end{tabular} & \begin{tabular}{|l}
$\mathrm{KAK}$ \\
$\mathrm{Na}$ \\
$\mathrm{cmol} / \mathrm{kg}$
\end{tabular} & $\begin{array}{l}\text { KAK } \\
\text { eff }\end{array}$ & $\begin{array}{l}\text { V- } \\
\text { wert } \\
\%\end{array}$ \\
\hline \multirow[t]{2}{*}{ Wald } & $\begin{array}{l}0- \\
30\end{array}$ & 6,82 & 6,48 & 4,69 & 0,38 & 12,20 & 8,1 & 1,0 & 24,9 & 0,0 & 1,0 & 3,3 & 0,0 & 0,0 & 28,3 & 96,6 \\
\hline & $\begin{array}{c}31- \\
60\end{array}$ & 5,20 & 4,28 & 1,79 & 0,13 & 13,98 & 3,1 & 1,8 & 0,2 & 0,0 & 0,2 & 1,1 & 0,0 & 0,0 & 2,7 & 32,0 \\
\hline $\begin{array}{l}\text { Alang } \\
\text { alang }\end{array}$ & $0-30$ & 4,95 & 4,02 & 1,33 & 0,09 & 15,10 & 2,2 & 1,4 & 0,2 & 0,0 & 0,2 & 0,2 & 0,0 & 0,0 & 2,1 & 28,9 \\
\hline
\end{tabular}

\begin{tabular}{lrrr}
\hline Parzellen & \multicolumn{1}{c}{$\begin{array}{l}\text { Tiefe } \\
(\mathrm{cm})\end{array}$} & $\begin{array}{c}\text { Vor dem Anbau } \\
\text { (P-Bray mg/kg Boden) }\end{array}$ & $\begin{array}{c}\text { Nach der Ernte } \\
\text { (P-Bray mg/kg Boden) }\end{array}$ \\
\hline 1.Jahr nach & $0-30$ & 3,63 & 0,50 \\
Brandrodung & $30-60$ & 0,69 & 0,42 \\
\hline 4. Jahr nach & $0-30$ & 0,62 & 0,59 \\
\cline { 2 - 4 } Brandrodung & $30-60$ & 0,56 & 0,87 \\
\hline \multirow{2}{*}{ 4 Jahre alte Brache } & $0-30$ & 0,88 & \\
\cline { 2 - 4 } Agroforstsystem & $30-60$ & 0,35 & \\
\cline { 2 - 4 } mit Mulch & $0-30$ & 0,74 & \\
\hline \multirow{2}{*}{ Wald } & $30-60$ & 0,30 & \\
\hline \multirow{2}{*}{ Alang Alang } & $0-30$ & 2,61 & \\
\cline { 2 - 4 } & $30-60$ & 0,72 & \\
\hline
\end{tabular}




\section{Oberboden}

$\mathrm{pH}(\mathrm{KCl})$ vor dem Anbau $(0-30 \mathrm{~cm})$

\begin{tabular}{|l|r|r|r|r|r|r|}
\hline & Wald & 1.Jahr & 4.Jahr & Brache & a.Alang & Agro.M \\
\hline Wald & & & & & & \\
\hline 1.Jahr & 10,64 & & & & & \\
\hline 4.Jahr & 2,45 & 7,13 & & & & \\
\hline Brache & 0,34 & 9,49 & 1,11 & & & \\
\hline a.Alang & 10,83 & 12,6 & 6,65 & 2,65 & & \\
\hline Agro.M & 3,56 & 14,22 & 12,02 & 7,23 & 2,21 & \\
\hline
\end{tabular}

$\mathrm{pH}(\mathrm{KCl})$ nach der Ernte $(0-30 \mathrm{~cm})$

\begin{tabular}{|l|r|r|r|r|}
\hline & 1.Jahr & 4.Jahr & Brache & Agro.M \\
\hline 1.Jahr & & & & \\
\hline 4.Jahr & 4,12 & & & \\
\hline Brache & 0,04 & 4,19 & & \\
\hline Agro.M & 8,79 & 6,26 & 8,88 & \\
\hline
\end{tabular}

\section{Oberboden}

C\% vor dem Anbau (0-30 cm)

\begin{tabular}{|l|r|r|r|r|r|r|}
\hline & Wald & $1 . J a h r$ & 4.Jahr & Brache & a.Alang & Agro.M \\
\hline Wald & & & & & & \\
\hline 1.Jahr & 1,54 & & & & & \\
\hline 4.Jahr & 4,73 & 0,36 & & & & \\
\hline Brache & 1,39 & 0,03 & 0,11 & & & \\
\hline a.Alang & 7,58 & 5,14 & 0,04 & 0,03 & & \\
\hline Agro.M & 10,4 & 5,9 & 4,38 & 4,74 & 4,66 & \\
\hline
\end{tabular}

$\mathrm{C} \%$ nach der Ernte $(0-30 \mathrm{~cm})$

\begin{tabular}{|l|r|r|r|r|}
\hline & 1.Jahr & 4.Jahr & Brache & Agro.M \\
\hline 1.Jahr & & & & \\
\hline 4.Jahr & 0,23 & & & \\
\hline Brache & 5,78 & 4,07 & & \\
\hline Agro.M & 0,11 & 0,04 & 5,41 & \\
\hline
\end{tabular}

\section{Oberboden}

N\% vor dem Anbau $(0-30 \mathrm{~cm})$

\begin{tabular}{|l|r|r|r|r|r|r|}
\hline & Wald & $1 . J a h r$ & $4 . J a h r$ & Brache & a.Alang & Agro.M \\
\hline Wald & & & & & & \\
\hline 1.Jahr & 0,05 & & & & & \\
\hline 4.Jahr & 0,24 & 0,68 & & & & \\
\hline Brache & 1,54 & 2,88 & 1,24 & & & \\
\hline a.Alang & 5,21 & 5,57 & 4 & 0,73 & & \\
\hline Agro.M & 0,51 & 1,14 & 0,11 & 0,5 & 2,29 & \\
\hline
\end{tabular}

N\% nach der Ernte $(0-30 \mathrm{~cm})$

\begin{tabular}{|l|r|r|r|r|}
\hline & 1.Jahr & 4.Jahr & Brache & Agro.M \\
\hline 1.Jahr & & & & \\
\hline 4.Jahr & 2,29 & & & \\
\hline Brache & 0,4 & 5 & & \\
\hline Agro.M & 10,32 & 6,72 & 11,7 & \\
\hline
\end{tabular}

\section{Unterboden}

$\mathrm{pH}(\mathrm{KCl})$ vor dem Anbau $(31-60 \mathrm{~cm})$

\begin{tabular}{|l|r|r|r|r|r|r|}
\hline & Wald & $1 . J a h r$ & $4 . J a h r$ & Brache & a.Alang & Agro.M \\
\hline Wald & & & & & & \\
\hline 1.Jahr & 3,54 & & & & & \\
\hline 4.Jahr & 8,33 & 6,19 & & & & \\
\hline Brache & 2,58 & 1,04 & 2,33 & & & \\
\hline a.Alang & 6,14 & 2,4 & 7,98 & 2,3 & & \\
\hline Agro.M & 0,05 & 3,27 & 8,19 & 2,46 & 0,04 & \\
\hline
\end{tabular}

$\mathrm{pH}(\mathrm{KCl})$ nach der Ernte $(31-60 \mathrm{~cm})$

\begin{tabular}{|l|r|r|r|r|}
\hline & 1.Jahr & 4.Jahr & Brache & Agro.M \\
\hline 1.Jahr & & & & \\
\hline 4.Jahr & 0,39 & & & \\
\hline Brache & 5,38 & 2,92 & & \\
\hline Agro.M & 1,57 & 0,35 & 1,88 & \\
\hline
\end{tabular}

\section{Unterboden}

C\% vor dem Anbau (31-60 cm)

\begin{tabular}{|l|r|r|r|r|r|r|}
\hline & Wald & 1.Jahr & 4.Jahr & Brache & a.Alang & Agro.M \\
\hline Wald & & & & & & \\
\hline 1.Jahr & 1,88 & & & & & \\
\hline 4.Jahr & 4,23 & 0,22 & & & & \\
\hline Brache & 0,07 & 1,25 & 3,02 & & & \\
\hline a.Alang & 6,35 & 1,03 & 2,77 & 0,06 & & \\
\hline Agro.M & 5,7 & 5,87 & 10,25 & 3,74 & 7,69 & \\
\hline
\end{tabular}

C\% nach der Ernte $(31-60 \mathrm{~cm})$

\begin{tabular}{|l|r|r|l|l|}
\hline & 1.Jahr & 4.Jahr & Brache & Agro.M \\
\hline 1.Jahr & & & & \\
\hline 4.Jahr & 0,23 & & & \\
\hline Brache & 0,02 & 0,07 & & \\
\hline Agro.M & 3,21 & 1,35 & 1,59 & \\
\hline
\end{tabular}

\section{Unterboden}

N\% vor dem Anbau (31-60 cm)

\begin{tabular}{|l|r|r|r|r|r|r|}
\hline & Wald & 1.Jahr & 4.Jahr & Brache & a.Alang & Agro.M \\
\hline Wald & & & & & & \\
\hline 1.Jahr & 1,6 & & & & & \\
\hline 4.Jahr & 0 & 1,6 & & & & \\
\hline Brache & 0,5 & 0,35 & 0,5 & & & \\
\hline a.Alang & 4,73 & 4,56 & 0,73 & 2,29 & & \\
\hline Agro.M & 0,18 & 1 & 0,18 & 0,14 & 2 & \\
\hline
\end{tabular}

N\% nach der Ernte $(31-60 \mathrm{~cm})$

\begin{tabular}{|l|r|r|l|r|}
\hline & 1.Jahr & 4.Jahr & Brache & Agro.M \\
\hline 1.Jahr & & & & \\
\hline 4.Jahr & 4,5 & & & \\
\hline Brache & 1,6 & 1 & & \\
\hline Agro.M & 2,48 & 0,64 & 0,09 & \\
\hline
\end{tabular}




\section{Oberboden}

$\mathrm{C} / \mathrm{N}$ vor dem Anbau $(0-30 \mathrm{~cm})$

\begin{tabular}{|l|r|r|r|r|r|r|}
\hline & Wald & 1 .Jahr & $4 . J a h r$ & Brache & a.Alang & Agro.M \\
\hline Wald & & & & & & \\
\hline 1.Jahr & 2,67 & & & & & \\
\hline 4.Jahr & 4,06 & 10,46 & & & & \\
\hline Brache & 2,4 & 9,34 & 0,42 & & & \\
\hline a.Alang & 6,17 & 5,49 & 5,48 & 5,54 & & \\
\hline Agro.M & 6,62 & 9,72 & 2,05 & 3,4 & 7,46 & \\
\hline
\end{tabular}

$\mathrm{C} / \mathrm{N}$ nach der Ernte $(0-30 \mathrm{~cm})$

\begin{tabular}{|l|r|r|r|r|}
\hline & 1.Jahr & 4.Jahr & Brache & Agro.M \\
\hline 1.Jahr & & & & \\
\hline 4.Jahr & 0,98 & & & \\
\hline Brache & 2,79 & 5,11 & & \\
\hline Agro.M & 0,54 & 0,18 & 6,96 & \\
\hline
\end{tabular}

\section{Oberboden}

KAKeff vor dem Anbau (0-30 cm)

\begin{tabular}{|l|r|r|r|r|r|r|}
\hline & Wald & $1 . J a h r$ & $4 . J a h r$ & Brache & a.Alang & Agro.M \\
\hline Wald & & & & & & \\
\hline 1.Jahr & 10,81 & & & & & \\
\hline 4.Jahr & 5,28 & 1,95 & & & & \\
\hline Brache & 5,23 & 6,99 & 1,94 & & & \\
\hline a.Alang & 7,17 & 12,33 & 6,88 & 12,02 & & \\
\hline Agro.M & 0,58 & 11,69 & 6,19 & 9,08 & 1,73 & \\
\hline
\end{tabular}

KAKeff nach der Ernte $(0-30 \mathrm{~cm})$

\begin{tabular}{|l|r|r|r|r|}
\hline & 1.Jahr & 4.Jahr & Brache & Agro.M \\
\hline 1.Jahr & & & & \\
\hline 4.Jahr & 0 & & & \\
\hline Brache & 0,06 & 0,06 & & \\
\hline Agro.M & 7,47 & 7,47 & 7,62 & \\
\hline
\end{tabular}

\section{Oberboden}

$\mathrm{Ca}(\mathrm{cmol} / \mathrm{kg})$ vor dem Anbau $(0-30 \mathrm{~cm})$

\begin{tabular}{|l|r|r|r|r|r|r|}
\hline & Wald & $1 . J a h r$ & $4 . J a h r$ & Brache & a.Alang & Agro.M \\
\hline Wald & & & & & & \\
\hline 1.Jahr & 6,55 & & & & & \\
\hline 4.Jahr & 7,19 & 0,15 & & & & \\
\hline Brache & 1,6 & 5,31 & 5,5 & & & \\
\hline a.Alang & 11,18 & 8,55 & 9,98 & 13,7 & & \\
\hline Agro.M & 0,09 & 6,79 & 7,53 & 2,48 & 14,74 & \\
\hline
\end{tabular}

$\mathrm{Ca}(\mathrm{cmol} / \mathrm{kg})$ nach der Ernte $(0-30 \mathrm{~cm})$

\begin{tabular}{|l|r|r|r|r|}
\hline & 1.Jahr & 4.Jahr & Brache & Agro.M \\
\hline 1.Jahr & & & & \\
\hline 4.Jahr & 0,87 & & & \\
\hline Brache & 2,88 & 3,35 & & \\
\hline Agro.M & 9,06 & 8,52 & 9,5 & \\
\hline
\end{tabular}

\section{Unterboden}

$\mathrm{C} / \mathrm{N}$ vor dem Anbau $(31-60 \mathrm{~cm})$

\begin{tabular}{|l|r|r|r|r|r|r|}
\hline & Wald & $1 . J a h r$ & $4 . J a h r$ & Brache & a.Alang & Agro.M \\
\hline Wald & & & & & & \\
\hline 1.Jahr & 0,01 & & & & & \\
\hline 4.Jahr & 3,76 & 3,52 & & & & \\
\hline Brache & 0,19 & 0,18 & 2,39 & & & \\
\hline a.Alang & 7,02 & 5,02 & 3,42 & 0,1 & & \\
\hline Agro.M & 8,47 & 8,33 & 3,17 & 7,34 & 8,21 & \\
\hline
\end{tabular}

$\mathrm{C} / \mathrm{N}$ nach der Ernte $(31-60 \mathrm{~cm})$

\begin{tabular}{|l|r|r|r|r|}
\hline & \multicolumn{1}{|l|}{ 1.Jahr } & 4.Jahr & Brache & Agro.M \\
\hline 1.Jahr & & & & \\
\hline 4.Jahr & 0,42 & & & \\
\hline Brache & 0,44 & 1,54 & & \\
\hline Agro.M & 4,52 & 3,17 & 5,73 & \\
\hline
\end{tabular}

\section{Unterboden}

KAKeff vor dem Anbau (31-60 cm)

\begin{tabular}{|l|r|r|r|r|r|r|}
\hline & Wald & 1 .Jahr & $4 . J a h r$ & Brache & a.Alang & Agro.M \\
\hline Wald & & & & & & \\
\hline 1.Jahr & 0,04 & & & & & \\
\hline 4.Jahr & 8,82 & 8,14 & & & & \\
\hline Brache & 2,54 & 2,31 & 0,4 & & & \\
\hline a.Alang & 5,57 & 4,75 & 12,95 & 4,3 & & \\
\hline Agro.M & 1,98 & 1,35 & 4,9 & 1,09 & 8,5 & \\
\hline
\end{tabular}

KAKeff nach der Ernte $(31-60 \mathrm{~cm})$

\begin{tabular}{|l|r|r|l|l|}
\hline & \multicolumn{1}{|l|}{ 1.Jahr } & 4.Jahr & Brache & Agro.M \\
\hline 1.Jahr & & & & \\
\hline 4.Jahr & 0,04 & & & \\
\hline Brache & 2,39 & 3,51 & & \\
\hline Agro.M & 3,91 & 5,46 & 12,2 & \\
\hline
\end{tabular}

\section{Unterboden}

$\mathrm{Ca}(\mathrm{cmol} / \mathrm{kg})$ vor dem Anbau $(31-60 \mathrm{~cm})$

\begin{tabular}{|l|r|r|r|r|r|r|}
\hline & Wald & $1 . J a h r$ & $4 . J a h r$ & Brache & a.Alang & Agro.M \\
\hline Wald & & & & & & \\
\hline 1.Jahr & 14,11 & & & & & \\
\hline 4.Jahr & 15,21 & 6,27 & & & & \\
\hline Brache & 8,69 & 1,26 & 1,96 & & & \\
\hline a.Alang & 10,73 & 13,76 & 15,09 & 8,57 & & \\
\hline Agro.M & 11,18 & 2,42 & 10,13 & 3,94 & 10,65 & \\
\hline
\end{tabular}

$\mathrm{Ca}(\mathrm{cmol} / \mathrm{kg})$ nach der Ernte $(31-60 \mathrm{~cm})$

\begin{tabular}{|l|r|r|l|l|}
\hline & 1.Jahr & 4.Jahr & Brache & Agro.M \\
\hline 1.Jahr & & & & \\
\hline 4.Jahr & 1,13 & & & \\
\hline Brache & 0,44 & 2,26 & & \\
\hline Agro.M & 6,13 & 2,62 & 6,13 & \\
\hline
\end{tabular}




\section{Oberboden}

$\mathrm{Mg}(\mathrm{cmol} / \mathrm{kg})$ vor dem Anbau $(0-30 \mathrm{~cm})$

\begin{tabular}{|l|r|r|r|r|r|r|}
\hline & Wald & 1.Jahr & 4.Jahr & Brache & a.Alang & Agro.M \\
\hline Wald & & & & & & \\
\hline 1.Jahr & 5,42 & & & & & \\
\hline 4.Jahr & 1,37 & 3,81 & & & & \\
\hline Brache & 0,79 & 3,54 & 0 & & & \\
\hline a.Alang & 12,74 & 7,7 & 10,66 & 4,37 & & \\
\hline Agro.M & 0,8 & 6,78 & 5,57 & 2,56 & 1,68 & \\
\hline
\end{tabular}

$\mathrm{Mg}(\mathrm{cmol} / \mathrm{kg})$ nach der Ernte $(0-30 \mathrm{~cm})$

\begin{tabular}{|l|r|r|r|r|}
\hline & 1.Jahr & 4.Jahr & Brache & Agro.M \\
\hline 1.Jahr & & & & \\
\hline 4.Jahr & 1,73 & & & \\
\hline Brache & 0,21 & 3,27 & & \\
\hline Agro.M & 5,11 & 8,29 & 4,05 & \\
\hline
\end{tabular}

\section{Oberboden}

$\mathrm{P}$-vor dem Anbau (0-30 cm)

\begin{tabular}{|l|r|r|r|r|r|r|}
\hline & Wald & 1 .Jahr & $4 . J a h r$ & Brache & a.Alang & Agro.M \\
\hline Wald & & & & & & \\
\hline 1.Jahr & 15,31 & & & & & \\
\hline 4.Jahr & 13,29 & 3,33 & & & & \\
\hline Brache & 10,36 & 1,49 & 0,34 & & & \\
\hline a.Alang & 6,9 & 12,16 & 7,71 & 6,99 & & \\
\hline Agro.M & 5,79 & 8,36 & 2,69 & 3,53 & 1,45 & \\
\hline
\end{tabular}

P- nach der Ernte $(0-30 \mathrm{~cm})$

\begin{tabular}{|l|r|r|r|r|}
\hline & 1.Jahr & 4.Jahr & Brache & Agro.M \\
\hline 1.Jahr & & & & \\
\hline 4.Jahr & 6,19 & & & \\
\hline Brache & 3,66 & 0,67 & & \\
\hline Agro.M & 5,88 & 2,16 & 3,39 & \\
\hline
\end{tabular}

\section{Oberboden}

K- vor dem Anbau (0-30 cm)

\begin{tabular}{|l|r|r|r|r|r|r|}
\hline & Wald & 1 .Jahr & 4.Jahr & Brache & a.Alang & Agro.M \\
\hline Wald & & & & & & \\
\hline 1.Jahr & 12,2 & & & & & \\
\hline 4.Jahr & 8,34 & 0,04 & & & & \\
\hline Brache & 15,28 & 0,77 & 0,87 & & & \\
\hline a.Alang & 12,99 & 12,17 & 8,32 & 15,22 & & \\
\hline Agro.M & 7,00 & 0,75 & 0,86 & 0,01 & 6,97 & \\
\hline
\end{tabular}

$\mathrm{K}$ - nach der Ernte $(0-30 \mathrm{~cm})$

\begin{tabular}{|l|r|r|r|r|}
\hline & 1.Jahr & 4.Jahr & Brache & Agro.M \\
\hline 1.Jahr & & & & \\
\hline 4.Jahr & 2,59 & & & \\
\hline Brache & 14,46 & 12,98 & & \\
\hline Agro.M & 2,94 & 1,99 & 5,17 & \\
\hline
\end{tabular}

\section{Unterboden}

$\mathrm{Mg}(\mathrm{cmol} / \mathrm{kg})$ vor dem Anbau $(31-60 \mathrm{~cm})$

\begin{tabular}{|l|r|r|r|r|r|r|}
\hline & Wald & $1 . J a h r$ & $4 . J a h r$ & Brache & a.Alang & Agro.M \\
\hline Wald & & & & & & \\
\hline 1.Jahr & 8,27 & & & & & \\
\hline 4.Jahr & 11,41 & 1,14 & & & & \\
\hline Brache & 3,39 & 0,16 & 0,14 & & & \\
\hline a.Alang & 8,35 & 7,72 & 10,95 & 3,23 & & \\
\hline Agro.M & 5,61 & 0,5 & 2,88 & 0,68 & 5,07 & \\
\hline
\end{tabular}

$\mathrm{Mg}(\mathrm{cmol} / \mathrm{kg})$ nach der Ernte $(31-60 \mathrm{~cm})$

\begin{tabular}{|l|r|r|r|r|}
\hline & 1.Jahr & 4.Jahr & Brache & Agro.M \\
\hline 1.Jahr & & & & \\
\hline 4.Jahr & 7,13 & & & \\
\hline Brache & 0,19 & 4,66 & & \\
\hline Agro.M & 4,97 & 0,5 & 2,95 & \\
\hline
\end{tabular}

\section{Unterboden}

P- vor dem Anbau (31-60 cm)

\begin{tabular}{|l|r|r|r|r|r|r|}
\hline & Wald & 1.Jahr & 4.Jahr & Brache & a.Alang & Agro.M \\
\hline Wald & & & & & & \\
\hline 1.Jahr & 14,54 & & & & & \\
\hline 4.Jahr & 8,66 & 1,99 & & & & \\
\hline Brache & 4,58 & 2,68 & 0,12 & & & \\
\hline a.Alang & 15,68 & 14,92 & 9,08 & 4,89 & & \\
\hline Agro.M & 11,19 & 9,49 & 3,69 & 1,6 & 13,02 & \\
\hline
\end{tabular}

P - nach der Ernte $(31-60 \mathrm{~cm})$

\begin{tabular}{|l|r|r|r|r|}
\hline & 1.Jahr & 4.Jahr & Brache & Agro.M \\
\hline 1.Jahr & & & & \\
\hline 4.Jahr & 1,75 & & & \\
\hline Brache & 0,39 & 0,51 & & \\
\hline Agro.M & 0,04 & 1,04 & 0,13 & \\
\hline
\end{tabular}

\section{Unterboden}

$\mathrm{K}$ - vor dem Anbau (31-60 cm)

\begin{tabular}{|l|r|r|r|r|r|r|}
\hline & Wald & $1 . J a h r$ & 4.Jahr & Brache & a.Alang & Agro.M \\
\hline Wald & & & & & & \\
\hline 1.Jahr & 7,66 & & & & & \\
\hline 4.Jahr & 15,86 & 0 & & & & \\
\hline Brache & 14,27 & 2,18 & 2,64 & & & \\
\hline a.Alang & 6,61 & 7,69 & 15,92 & 14,29 & & \\
\hline Agro.M & 9,24 & 0,33 & 0,51 & 4,3 & 9,28 & \\
\hline
\end{tabular}

K- nach der Ernte $(31-60 \mathrm{~cm})$

\begin{tabular}{|l|r|r|r|r|}
\hline & 1.Jahr & 4.Jahr & Brache & Agro.M \\
\hline 1.Jahr & & & & \\
\hline 4.Jahr & 0,51 & & & \\
\hline Brache & 0,02 & 1,14 & & \\
\hline Agro.M & 0,77 & 3,66 & 0,79 & \\
\hline
\end{tabular}




\section{Oberboden}

$\mathrm{Ca}$ - vor dem Anbau (0-30 cm)

\begin{tabular}{|l|r|r|r|r|r|r|}
\hline & Wald & 1 .Jahr & $4 . J a h r$ & Brache & a.Alang & Agro.M \\
\hline Wald & & & & & & \\
\hline 1.Jahr & 6,75 & & & & & \\
\hline 4.Jahr & 10,77 & 0,21 & & & & \\
\hline Brache & 11,07 & 2,05 & 1,86 & & & \\
\hline a.Alang & 7,81 & 6,94 & 11,16 & 11,84 & & \\
\hline Agro.M & 6,41 & 5,3 & 7,77 & 5,47 & 8,28 & \\
\hline
\end{tabular}

Ca- nach der Ernte $(0-30 \mathrm{~cm})$

\begin{tabular}{|l|r|r|r|r|}
\hline & 1.Jahr & 4.Jahr & Brache & Agro.M \\
\hline 1.Jahr & & & & \\
\hline 4.Jahr & 1,23 & & & \\
\hline Brache & 12,36 & 8,53 & & \\
\hline Agro.M & 7,61 & 7,27 & 5,06 & \\
\hline
\end{tabular}

\section{Oberboden}

$\mathrm{Mg}$-vor dem Anbau (0-30 cm)

\begin{tabular}{|l|r|r|r|r|r|r|}
\hline & Wald & $1 . J a h r$ & $4 . J a h r$ & Brache & a.Alang & Agro.M \\
\hline Wald & & & & & & \\
\hline 1.Jahr & 11,42 & & & & & \\
\hline 4.Jahr & 14,81 & 5,72 & & & & \\
\hline Brache & 15,67 & 2,76 & 1,25 & & & \\
\hline a.Alang & 10,04 & 11,41 & 14,81 & 15,66 & & \\
\hline Agro.M & 15,67 & 2,61 & 10,16 & 8,38 & 15,64 & \\
\hline
\end{tabular}

$\mathrm{Mg}$-nach der Ernte $(0-30 \mathrm{~cm})$

\begin{tabular}{|l|r|r|r|r|}
\hline & 1.Jahr & 4.Jahr & Brache & Agro.M \\
\hline 1.Jahr & & & & \\
\hline 4.Jahr & 4,97 & & & \\
\hline Brache & 13,92 & 15,53 & & \\
\hline Agro.M & 4,11 & 7,24 & 8,44 & \\
\hline
\end{tabular}

\section{Oberboden}

$\mathrm{Na}$-vor dem Anbau (0-30 cm)

\begin{tabular}{|l|r|r|r|r|r|r|}
\hline & Wald & 1 .Jahr & 4.Jahr & Brache & a.Alang & Agro.M \\
\hline Wald & & & & & & \\
\hline 1.Jahr & 10,07 & & & & & \\
\hline 4.Jahr & 13,78 & 0,01 & & & & \\
\hline Brache & 15,9 & 15,27 & 15,26 & & & \\
\hline a.Alang & 15,86 & 9,42 & 12,88 & 15,88 & & \\
\hline Agro.M & 12,88 & 4,39 & 4,5 & 14,19 & 12,58 & \\
\hline
\end{tabular}

Na- nach der Ernte $(0-30 \mathrm{~cm})$

\begin{tabular}{|l|r|r|r|r|}
\hline & 1.Jahr & 4.Jahr & Brache & Agro.M \\
\hline 1.Jahr & & & & \\
\hline 4.Jahr & 5,97 & & & \\
\hline Brache & 14,23 & 9,4 & & \\
\hline Agro.M & 6,15 & 3,65 & 0,34 & \\
\hline
\end{tabular}

\section{Unterboden}

$\mathrm{Ca}$ - vor dem Anbau (31-60 cm)

\begin{tabular}{|l|r|r|r|r|r|r|}
\hline & Wald & $1 . J a h r$ & $4 . J a h r$ & Brache & a.Alang & Agro.M \\
\hline Wald & & & & & & \\
\hline 1.Jahr & 15,27 & & & & & \\
\hline 4.Jahr & 15,71 & 4,46 & & & & \\
\hline Brache & 11,16 & 0,4 & 2,31 & & & \\
\hline a.Alang & 8,68 & 15,76 & 15,93 & 11,43 & & \\
\hline Agro.M & 6,24 & 8,16 & 12,59 & 7,22 & 7,18 & \\
\hline
\end{tabular}

$\mathrm{Ca}$ - nach der Ernte $(31-60 \mathrm{~cm})$

\begin{tabular}{|l|r|r|l|r|}
\hline & 1.Jahr & 4.Jahr & Brache & Agro.M \\
\hline 1.Jahr & & & & \\
\hline 4.Jahr & 1,2 & & & \\
\hline Brache & 0,28 & 0,32 & & \\
\hline Agro.M & 5,3 & 7,46 & 5,6 & \\
\hline
\end{tabular}

\section{Unterboden}

$\mathrm{Mg}$ - vor dem Anbau (31-60 cm)

\begin{tabular}{|l|r|r|r|r|r|r|}
\hline & Wald & $1 . J a h r$ & $4 . J a h r$ & Brache & a.Alang & Agro.M \\
\hline Wald & & & & & & \\
\hline 1.Jahr & 15,58 & & & & & \\
\hline 4.Jahr & 15,27 & 9,8 & & & & \\
\hline Brache & 10,54 & 4,28 & 2,63 & & & \\
\hline a.Alang & 11,40 & 15,58 & 15,27 & 10,54 & & \\
\hline Agro.M & 8,68 & 13,82 & 6,73 & 7,73 & 8,66 & \\
\hline
\end{tabular}

Mg- nach der Ernte $(31-60 \mathrm{~cm})$

\begin{tabular}{|l|r|r|l|l|}
\hline & \multicolumn{1}{|l|}{ 1.Jahr } & 4.Jahr & Brache & Agro.M \\
\hline 1.Jahr & & & & \\
\hline 4.Jahr & 2,54 & & & \\
\hline Brache & 7,43 & 0,94 & & \\
\hline Agro.M & 5,55 & 6,98 & 14,14 & \\
\hline
\end{tabular}

\section{Unterboden}

$\mathrm{Na}$ - vor dem Anbau (31-60 cm)

\begin{tabular}{|l|r|r|r|r|r|r|}
\hline & Wald & $1 . J a h r$ & $4 . J a h r$ & Brache & a.Alang & Agro.M \\
\hline Wald & & & & & & \\
\hline 1.Jahr & 11,57 & & & & & \\
\hline 4.Jahr & 12,82 & 2,47 & & & & \\
\hline Brache & 8,09 & 0,81 & 4,84 & & & \\
\hline a.Alang & 10,19 & 11,81 & 12,95 & 8,35 & & \\
\hline Agro.M & 5,43 & 0,83 & 0,08 & 1,87 & 5,49 & \\
\hline
\end{tabular}

$\mathrm{Na}$ - nach der Ernte $(31-60 \mathrm{~cm})$

\begin{tabular}{|l|r|r|l|r|}
\hline & 1.Jahr & 4.Jahr & Brache & Agro.M \\
\hline 1.Jahr & & & & \\
\hline 4.Jahr & 0,12 & & & \\
\hline Brache & 1,82 & 2,5 & & \\
\hline Agro.M & 0,72 & 1,37 & 0,47 & \\
\hline
\end{tabular}




\section{Oberboden}

Fe -vor dem Anbau (0-30 cm)

\begin{tabular}{|l|r|r|r|r|r|r|}
\hline & Wald & 1.Jahr & 4.Jahr & Brache & a.Alang & Agro.M \\
\hline Wald & & & & & & \\
\hline 1.Jahr & 11,93 & & & & & \\
\hline 4.Jahr & 13,69 & 3,39 & & & & \\
\hline Brache & 7,74 & 1,6 & 0,08 & & & \\
\hline a.Alang & 13,01 & 11,9 & 13,68 & 7,73 & & \\
\hline Agro.M & 9,93 & 5,21 & 1,47 & 1,85 & 9,92 & \\
\hline
\end{tabular}

Fe - nach der Ernte $(0-30 \mathrm{~cm})$

\begin{tabular}{|l|r|r|r|r|}
\hline & 1.Jahr & 4.Jahr & Brache & Agro.M \\
\hline 1.Jahr & & & & \\
\hline 4.Jahr & 2,46 & & & \\
\hline Brache & 5,78 & 2,12 & & \\
\hline Agro.M & 0,83 & 4,61 & 7,33 & \\
\hline
\end{tabular}

\section{Oberboden}

$\mathrm{Al}$-vor dem Anbau $(0-30 \mathrm{~cm})$

\begin{tabular}{|l|r|r|r|r|r|r|}
\hline & Wald & $1 . J a h r$ & $4 . J a h r$ & Brache & a.Alang & Agro.M \\
\hline Wald & & & & & & \\
\hline 1.Jahr & 10,79 & & & & & \\
\hline 4.Jahr & 6,62 & 1,82 & & & & \\
\hline Brache & 4,19 & 1 & 0,02 & & & \\
\hline a.Alang & 10,13 & 10,79 & 6,62 & 4,19 & & \\
\hline Agro.M & 9,8 & 3,39 & 0,12 & 0,22 & 9,8 & \\
\hline
\end{tabular}

$\mathrm{Al}$ - nach der Ernte $(0-30 \mathrm{~cm})$

\begin{tabular}{|l|r|r|r|r|}
\hline & 1.Jahr & 4.Jahr & Brache & Agro.M \\
\hline 1.Jahr & & & & \\
\hline 4.Jahr & 1,2 & & & \\
\hline Brache & 0,04 & 1,28 & & \\
\hline Agro.M & 0,47 & 3,76 & 0,96 & \\
\hline
\end{tabular}

\section{Oberboden}

Mn - vor dem Anbau (0-30 cm)

\begin{tabular}{|l|r|r|r|r|r|r|}
\hline & Wald & 1 .Jahr & $4 . J a h r$ & Brache & a.Alang & Agro.M \\
\hline Wald & & & & & & \\
\hline 1.Jahr & 8,58 & & & & & \\
\hline 4.Jahr & 5,45 & 1,59 & & & & \\
\hline Brache & 4,21 & 0,47 & 0,36 & & & \\
\hline a.Alang & 9,06 & 8,5 & 5,43 & 4,19 & & \\
\hline Agro.M & 8,74 & 4,14 & 0,59 & 1,88 & 8,71 & \\
\hline
\end{tabular}

Mn - nach der Ernte (0-30 cm)

\begin{tabular}{|l|r|r|r|r|}
\hline & 1.Jahr & 4.Jahr & Brache & Agro.M \\
\hline 1.Jahr & & & & \\
\hline 4.Jahr & 0,16 & & & \\
\hline Brache & 7,99 & 7,04 & & \\
\hline Agro.M & 4,74 & 4,03 & 0,21 & \\
\hline
\end{tabular}

\section{Unterboden}

Fe - vor dem Anbau (31-60 cm)

\begin{tabular}{|l|r|r|r|r|r|r|}
\hline & Wald & $1 . J a h r$ & $4 . J a h r$ & Brache & a.Alang & Agro.M \\
\hline Wald & & & & & & \\
\hline 1.Jahr & 11,99 & & & & & \\
\hline 4.Jahr & 15,1 & 0,99 & & & & \\
\hline Brache & 14,04 & 2,84 & 6,33 & & & \\
\hline a.Alang & 12,61 & 11,97 & 15,09 & 13,98 & & \\
\hline Agro.M & 9,55 & 1,55 & 4,55 & 0,22 & 9,52 & \\
\hline
\end{tabular}

Fe- nach der Ernte $(31-60 \mathrm{~cm})$

\begin{tabular}{|l|r|r|r|r|}
\hline & \multicolumn{1}{|l|}{ 1.Jahr } & 4.Jahr & Brache & Agro.M \\
\hline 1.Jahr & & & & \\
\hline 4.Jahr & 0,01 & & & \\
\hline Brache & 0,02 & 0,04 & & \\
\hline Agro.M & 0,46 & 0,57 & 0,24 & \\
\hline
\end{tabular}

\section{Unterboden}

$\mathrm{Al}$ - vor dem Anbau (31-60 cm)

\begin{tabular}{|l|r|r|r|r|r|r|}
\hline & Wald & 1.Jahr & 4.Jahr & Brache & a.Alang & Agro.M \\
\hline Wald & & & & & & \\
\hline 1.Jahr & 9,44 & & & & & \\
\hline 4.Jahr & 11,7 & 4,54 & & & & \\
\hline Brache & 9,11 & 0,04 & 5,05 & & & \\
\hline a.Alang & 10,87 & 9,44 & 11,69 & 9,11 & & \\
\hline Agro.M & 11,72 & 0,01 & 4,83 & 0,02 & 11,71 & \\
\hline
\end{tabular}

Al- nach der Ernte $(31-60 \mathrm{~cm})$

\begin{tabular}{|l|r|r|r|r|}
\hline & \multicolumn{1}{|l|}{ 1.Jahr } & 4.Jahr & Brache & Agro.M \\
\hline 1.Jahr & & & & \\
\hline 4.Jahr & 1,3 & & & \\
\hline Brache & 0,69 & 0,13 & & \\
\hline Agro.M & 0,81 & 0,06 & 0,01 & \\
\hline
\end{tabular}

\section{Unterboden}

Mn - vor dem Anbau (31-60 cm)

\begin{tabular}{|l|r|r|r|r|r|r|}
\hline & Wald & $1 . J a h r$ & $4 . J a h r$ & Brache & a.Alang & Agro.M \\
\hline Wald & & & & & & \\
\hline 1.Jahr & 14,39 & & & & & \\
\hline 4.Jahr & 14,16 & 4,76 & & & & \\
\hline Brache & 14,51 & 5,48 & 0,07 & & & \\
\hline a.Alang & 15,39 & 14,34 & 14,04 & 14,37 & & \\
\hline Agro.M & 9,88 & 7,97 & 1,55 & 1,05 & 9,74 & \\
\hline
\end{tabular}

Mn - nach der Ernte $(31-60 \mathrm{~cm})$

\begin{tabular}{|l|r|r|l|r|}
\hline & \multicolumn{1}{|l|}{ 1.Jahr } & 4.Jahr & Brache & Agro.M \\
\hline 1.Jahr & & & & \\
\hline 4.Jahr & 1,01 & & & \\
\hline Brache & 1,22 & 0,02 & & \\
\hline Agro.M & 0,34 & 2,28 & 2,48 & \\
\hline
\end{tabular}




\section{Oberboden}

Mo - vor dem Anbau (0-30 cm)

\begin{tabular}{|l|r|r|r|r|r|r|}
\hline & Wald & 1 .Jahr & $4 . J a h r$ & Brache & a.Alang & Agro.M \\
\hline Wald & & & & & & \\
\hline 1.Jahr & 14,51 & & & & & \\
\hline 4.Jahr & 14,26 & 1,43 & & & & \\
\hline Brache & 5,81 & 9,65 & 6,87 & & & \\
\hline a.Alang & 16,40 & 14,52 & 14,28 & 5,84 & & \\
\hline Agro.M & 6,6 & 12,71 & 11,41 & 1,98 & 6,69 & \\
\hline
\end{tabular}

Mo - nach der Ernte $(0-30 \mathrm{~cm})$

\begin{tabular}{|l|r|r|r|r|}
\hline & 1.Jahr & 4.Jahr & Brache & Agro.M \\
\hline 1.Jahr & & & & \\
\hline 4.Jahr & 0,73 & & & \\
\hline Brache & 10,24 & 10,81 & & \\
\hline Agro.M & 8,95 & 9,85 & 1,45 & \\
\hline
\end{tabular}

\section{Oberboden}

$\mathrm{Pb}$ - vor dem Anbau (0-30 cm)

\begin{tabular}{|l|r|r|r|r|r|r|}
\hline & Wald & $1 . J a h r$ & $4 . J a h r$ & Brache & a.Alang & Agro.M \\
\hline Wald & & & & & & \\
\hline 1.Jahr & 9,1 & & & & & \\
\hline 4.Jahr & 14,62 & 0,48 & & & & \\
\hline Brache & 15,98 & 14,65 & 14,23 & & & \\
\hline a.Alang & 7,23 & 7,4 & 12,51 & 15,85 & & \\
\hline Agro.M & 13,29 & 5,34 & 9,86 & 15,68 & 3,38 & \\
\hline
\end{tabular}

$\mathrm{Pb}$ nach der Ernte $(0-30 \mathrm{~cm})$

\begin{tabular}{|l|r|r|r|r|}
\hline & 1.Jahr & 4.Jahr & Brache & Agro.M \\
\hline 1.Jahr & & & & \\
\hline 4.Jahr & 0,16 & & & \\
\hline Brache & 0,46 & 0,1 & & \\
\hline Agro.M & 0,11 & 0,78 & 1,46 & \\
\hline
\end{tabular}

\section{Oberboden}

Zn vor dem Anbau (0-30 cm)

\begin{tabular}{|l|r|r|r|r|r|r|}
\hline & Wald & $1 . J a h r$ & $4 . J a h r$ & Brache & a.Alang & Agro.M \\
\hline Wald & & & & & & \\
\hline 1.Jahr & 13,76 & & & & & \\
\hline 4.Jahr & 9,99 & 7,88 & & & & \\
\hline Brache & 7,02 & 6,23 & 0,22 & & & \\
\hline a.Alang & 14,4 & 13,27 & 8,12 & 6,08 & & \\
\hline Agro.M & 7,78 & 7,41 & 0,02 & 0,12 & 6,49 & \\
\hline
\end{tabular}

Zn- nach der Ernte $(0-30 \mathrm{~cm})$

\begin{tabular}{|l|r|r|r|r|}
\hline & 1.Jahr & 4.Jahr & Brache & Agro.M \\
\hline 1.Jahr & & & & \\
\hline 4.Jahr & 0,62 & & & \\
\hline Brache & 11,13 & 7,81 & & \\
\hline Agro.M & 3,84 & 2,81 & 0,04 & \\
\hline
\end{tabular}

\section{Unterboden}

Mo - vor dem Anbau (31-60 cm)

\begin{tabular}{|l|r|r|r|r|r|r|}
\hline & Wald & 1.Jahr & 4.Jahr & Brache & a.Alang & Agro.M \\
\hline Wald & & & & & & \\
\hline 1.Jahr & 7,77 & & & & & \\
\hline 4.Jahr & 7,02 & 0,17 & & & & \\
\hline Brache & 14,42 & 8,2 & 6,75 & & & \\
\hline a.Alang & 13,21 & 7,41 & 6,75 & 14,28 & & \\
\hline Agro.M & 9,57 & 3,03 & 3,46 & 12,26 & 8,25 & \\
\hline
\end{tabular}

Mo- nach der Ernte $(31-60 \mathrm{~cm})$

\begin{tabular}{|l|r|r|l|r|}
\hline & 1.Jahr & 4.Jahr & Brache & Agro.M \\
\hline 1.Jahr & & & & \\
\hline 4.Jahr & 4,68 & & & \\
\hline Brache & 0,15 & 5,54 & & \\
\hline Agro.M & 12,18 & 10,33 & 12,36 & \\
\hline
\end{tabular}

\section{Unterboden}

$\mathrm{Pb}$ - vor dem Anbau (31-60 cm)

\begin{tabular}{|l|r|r|r|r|r|r|}
\hline & Wald & 1 .Jahr & $4 . J a h r$ & Brache & a.Alang & Agro.M \\
\hline Wald & & & & & & \\
\hline 1.Jahr & 15,2 & & & & & \\
\hline 4.Jahr & 12,28 & 11,03 & & & & \\
\hline Brache & 7,12 & 14,14 & 6,37 & & & \\
\hline a.Alang & 11,64 & 15,39 & 13,48 & 10,7 & & \\
\hline Agro.M & 3,53 & 14,49 & 7,95 & 0,47 & 6,19 & \\
\hline
\end{tabular}

$\mathrm{Pb}$ nach der Ernte $(31-60 \mathrm{~cm})$

\begin{tabular}{|l|r|r|l|r|}
\hline & 1.Jahr & 4.Jahr & Brache & Agro.M \\
\hline 1.Jahr & & & & \\
\hline 4.Jahr & 3,49 & & & \\
\hline Brache & 8,41 & 6,16 & & \\
\hline Agro.M & 0,01 & 3,24 & 4,38 & \\
\hline
\end{tabular}

\section{Unterboden}

$\mathrm{Zn}$ vor dem Anbau (31-60 cm)

\begin{tabular}{|l|r|r|r|r|r|r|}
\hline & Wald & 1.Jahr & 4.Jahr & Brache & a.Alang & Agro.M \\
\hline Wald & & & & & & \\
\hline 1.Jahr & 13,77 & & & & & \\
\hline 4.Jahr & 4,64 & 8,69 & & & & \\
\hline Brache & 10,51 & 10,74 & 0,51 & & & \\
\hline a.Alang & 12,33 & 13,95 & 5 & 12,19 & & \\
\hline Agro.M & 5,36 & 6,95 & 0,27 & 1,41 & 5,65 & \\
\hline
\end{tabular}

Zn - nach der Ernte $(31-60 \mathrm{~cm})$

\begin{tabular}{|l|r|r|l|r|}
\hline & 1.Jahr & 4.Jahr & Brache & Agro.M \\
\hline 1.Jahr & & & & \\
\hline 4.Jahr & 0 & & & \\
\hline Brache & 0,14 & 0,17 & & \\
\hline Agro.M & 7,7 & 8,57 & 3,8 & \\
\hline
\end{tabular}




\section{Oberboden}

Co - vor dem Anbau (0-30 cm)

\begin{tabular}{|l|r|r|r|r|r|r|}
\hline & Wald & $1 . J a h r$ & $4 . J a h r$ & Brache & a.Alang & Agro.M \\
\hline Wald & & & & & & \\
\hline 1.Jahr & 9,91 & & & & & \\
\hline 4.Jahr & 13,34 & 1,48 & & & & \\
\hline Brache & 4,72 & 4,33 & 1,96 & & & \\
\hline a.Alang & 12,34 & 8,23 & 9,12 & 2,2 & & \\
\hline Agro.M & 8,22 & 0,01 & 1,47 & 3,88 & 6,91 & \\
\hline
\end{tabular}

Co - nach der Ernte $(0-30 \mathrm{~cm})$

\begin{tabular}{|l|r|r|r|r|}
\hline & 1.Jahr & 4.Jahr & Brache & Agro.M \\
\hline 1.Jahr & & & & \\
\hline 4.Jahr & 0,46 & & & \\
\hline Brache & 3,52 & 4,84 & & \\
\hline Agro.M & 0,91 & 2,07 & 1,25 & \\
\hline
\end{tabular}

\section{Oberboden}

$\mathrm{Cu}$ - vor dem Anbau (0-30 cm)

\begin{tabular}{|l|r|r|r|r|r|r|}
\hline & Wald & $1 . J a h r$ & $4 . J a h r$ & Brache & a.Alang & Agro.M \\
\hline Wald & & & & & & \\
\hline 1.Jahr & 10,5 & & & & & \\
\hline 4.Jahr & 4,36 & 0,71 & & & & \\
\hline Brache & 8,55 & 0,21 & 0,3 & & & \\
\hline a.Alang & 12,28 & 5,3 & 3,22 & 5,2 & & \\
\hline Agro.M & 12,77 & 1,04 & 0,05 & 0,25 & 8,47 & \\
\hline
\end{tabular}

$\mathrm{Cu}$ - nach der Ernte $(0-30 \mathrm{~cm})$

\begin{tabular}{|l|r|r|r|r|}
\hline & 1.Jahr & 4.Jahr & Brache & Agro.M \\
\hline 1.Jahr & & & & \\
\hline 4.Jahr & 9,61 & & & \\
\hline Brache & 2,68 & 7,51 & & \\
\hline Agro.M & 0,18 & 8,55 & 3,36 & \\
\hline
\end{tabular}

\section{Unterboden}

Co - vor dem Anbau (31-60 cm)

\begin{tabular}{|l|r|r|r|r|r|r|}
\hline & Wald & $1 . J a h r$ & $4 . J a h r$ & Brache & a.Alang & Agro.M \\
\hline Wald & & & & & & \\
\hline $1 . J a h r$ & 14,19 & & & & & \\
\hline 4.Jahr & 13,6 & 8,71 & & & & \\
\hline Brache & 5,43 & 3,24 & 1,37 & & & \\
\hline a.Alang & 15,01 & 14,49 & 15,11 & 5,71 & & \\
\hline Agro.M & 4,16 & 1,72 & 1,5 & 0,1 & 4,33 & \\
\hline
\end{tabular}

Co - nach der Ernte $(31-60 \mathrm{~cm})$

\begin{tabular}{|l|r|r|r|r|}
\hline & 1.Jahr & 4.Jahr & Brache & Agro.M \\
\hline 1.Jahr & & & & \\
\hline 4.Jahr & 3,63 & & & \\
\hline Brache & 0,12 & 3,91 & & \\
\hline Agro.M & 0,22 & 4,17 & 0,64 & \\
\hline
\end{tabular}

\section{Unterboden}

$\mathrm{Cu}$ - vor dem Anbau (31-60 cm)

\begin{tabular}{|l|r|r|r|r|r|r|}
\hline & Wald & $1 . J a h r$ & $4 . J a h r$ & Brache & a.Alang & Agro.M \\
\hline Wald & & & & & & \\
\hline 1.Jahr & 11,57 & & & & & \\
\hline 4.Jahr & 11,01 & 0,15 & & & & \\
\hline Brache & 2,5 & 4,67 & 3,63 & & & \\
\hline a.Alang & 11,29 & 12,98 & 12,63 & 3,68 & & \\
\hline Agro.M & 9,09 & 2,38 & 1,48 & 0,89 & 11,68 & \\
\hline
\end{tabular}

$\mathrm{Cu}$ - nach der Ernte $(31-60 \mathrm{~cm})$

\begin{tabular}{|l|r|r|l|r|}
\hline & 1.Jahr & 4.Jahr & Brache & Agro.M \\
\hline 1.Jahr & & & & \\
\hline 4.Jahr & 3,33 & & & \\
\hline Brache & 3,96 & 0,08 & & \\
\hline Agro.M & 3,28 & 12,91 & 12,7 & \\
\hline
\end{tabular}


(t-Test) vor Anbau und nach der Ernte im Oberboden $(0-30 \mathrm{~cm})$

\begin{tabular}{|l|l|l|l|l|l|l|l|l|l|l|}
\hline Pazelle & $\begin{array}{l}\mathrm{pH} \\
(\mathrm{KCl})\end{array}$ & $\mathrm{C} \%$ & $\mathrm{~N} \%$ & $\mathrm{C} / \mathrm{N}$ & KAKeff & $\begin{array}{l}\mathrm{Ca} \\
(\mathrm{cmol} / \mathrm{kg})\end{array}$ & $\begin{array}{l}\mathrm{Mg} \\
(\mathrm{cmol} / \mathrm{kg})\end{array}$ & $\mathrm{P}$ & $\mathrm{K}$ & $\mathrm{Ca}$ \\
\hline 1.Jahr & 13,18 & 4,72 & 5,57 & 6,37 & 9,42 & 6,13 & 5,42 & 2,64 & 3,58 & 4,61 \\
\hline 4.Jahr & 0,57 & 2,82 & 0,12 & 2,74 & 3,95 & 4,44 & 8,53 & 6,70 & 0,51 & 2,34 \\
\hline Brache & 4,23 & 0,14 & 2,57 & 0,88 & 3,12 & 5,26 & 0,25 & 2,64 & 13,29 & 8,01 \\
\hline Agro.M & 9,41 & 0,01 & 6,72 & 0,03 & 8,20 & 9,28 & 7,40 & 6,89 & 2,21 & 7,71 \\
\hline
\end{tabular}

\begin{tabular}{|l|l|l|l|l|l|l|l|l|l|l|}
\hline Pazelle & $\mathrm{Mg}$ & $\mathrm{Na}$ & $\mathrm{Fe}$ & $\mathrm{Al}$ & $\mathrm{Mn}$ & $\mathrm{Mo}$ & $\mathrm{Pb}$ & $\mathrm{Zn}$ & $\mathrm{Co}$ & $\mathrm{Cu}$ \\
\hline 1.Jahr & 0,11 & 7,46 & 5,15 & 4,07 & 7,11 & 13,75 & 0,01 & 4,78 & 0,32 & 1,73 \\
\hline 4.Jahr & 12,19 & 0,21 & 0,69 & 0,08 & 4,57 & 13,40 & 0,05 & 1,67 & 1,62 & 3,87 \\
\hline Brache & 12,62 & 11,54 & 2,04 & 0,62 & 13,33 & 1,95 & 14,16 & 11,98 & 7,39 & 4,05 \\
\hline Agro.M & 6,46 & 1,43 & 0,23 & 1,49 & 6,77 & 4,76 & 7,47 & 4,35 & 1,64 & 0,00 \\
\hline
\end{tabular}

\section{(t- Test) vor Anbau und nach der Ernte im Unterboden (31-60 cm)}

\begin{tabular}{|l|l|l|l|l|l|l|l|l|l|l|}
\hline Pazelle & $\begin{array}{l}\mathrm{pH} \\
(\mathrm{KCl})\end{array}$ & $\mathrm{C} \%$ & $\mathrm{~N} \%$ & $\mathrm{C} / \mathrm{N}$ & KAKeff & $\begin{array}{l}\mathrm{Ca} \\
(\mathrm{cmol} / \mathrm{kg})\end{array}$ & $\begin{array}{l}\mathrm{Mg} \\
(\mathrm{cmol} / \mathrm{kg})\end{array}$ & $\mathrm{P}$ & $\mathrm{K}$ & $\mathrm{Ca}$ \\
\hline 1.Jahr & 5,53 & 0,00 & 1,60 & 5,14 & 2,78 & 2,86 & 4,97 & 3,70 & 0,28 & 2,32 \\
\hline 4.Jahr & 2,7 & 0,85 & 0,18 & 0,58 & 2,34 & 4,23 & 2,88 & 0,66 & 2,81 & 0,16 \\
\hline Brache & 1,09 & 0,95 & 0,35 & 5,69 & 0,91 & 0,78 & 1,14 & 0,16 & 3,97 & 1,55 \\
\hline Agro.M & 7,4 & 3,57 & 0,64 & 7,42 & 4,63 & 0,25 & 0,50 & 1,49 & 1,02 & 4,93 \\
\hline
\end{tabular}

\begin{tabular}{|l|l|l|l|l|l|l|l|l|l|l|}
\hline Pazelle & $\mathrm{Mg}$ & $\mathrm{Na}$ & $\mathrm{Fe}$ & $\mathrm{Al}$ & $\mathrm{Mn}$ & $\mathrm{Mo}$ & $\mathrm{Pb}$ & $\mathrm{Zn}$ & $\mathrm{Co}$ & $\mathrm{Cu}$ \\
\hline 1.Jahr & 11,99 & 0,05 & 2,34 & 0,61 & 15,14 & 14,62 & 14,24 & 6,52 & 2,70 & 0,11 \\
\hline 4.Jahr & 0,63 & 2,55 & 5,88 & 3,14 & 0,00 & 0,76 & 0,01 & 1,05 & 0,80 & 4,15 \\
\hline Brache & 0,00 & 3,67 & 0,06 & 0,09 & 0,16 & 12,68 & 9,05 & 1,57 & 0,01 & 9,80 \\
\hline Agro.M & 2,84 & 0,01 & 0,10 & 0,09 & 0,03 & 12,08 & 0,24 & 3,34 & 0,08 & 6,96 \\
\hline
\end{tabular}



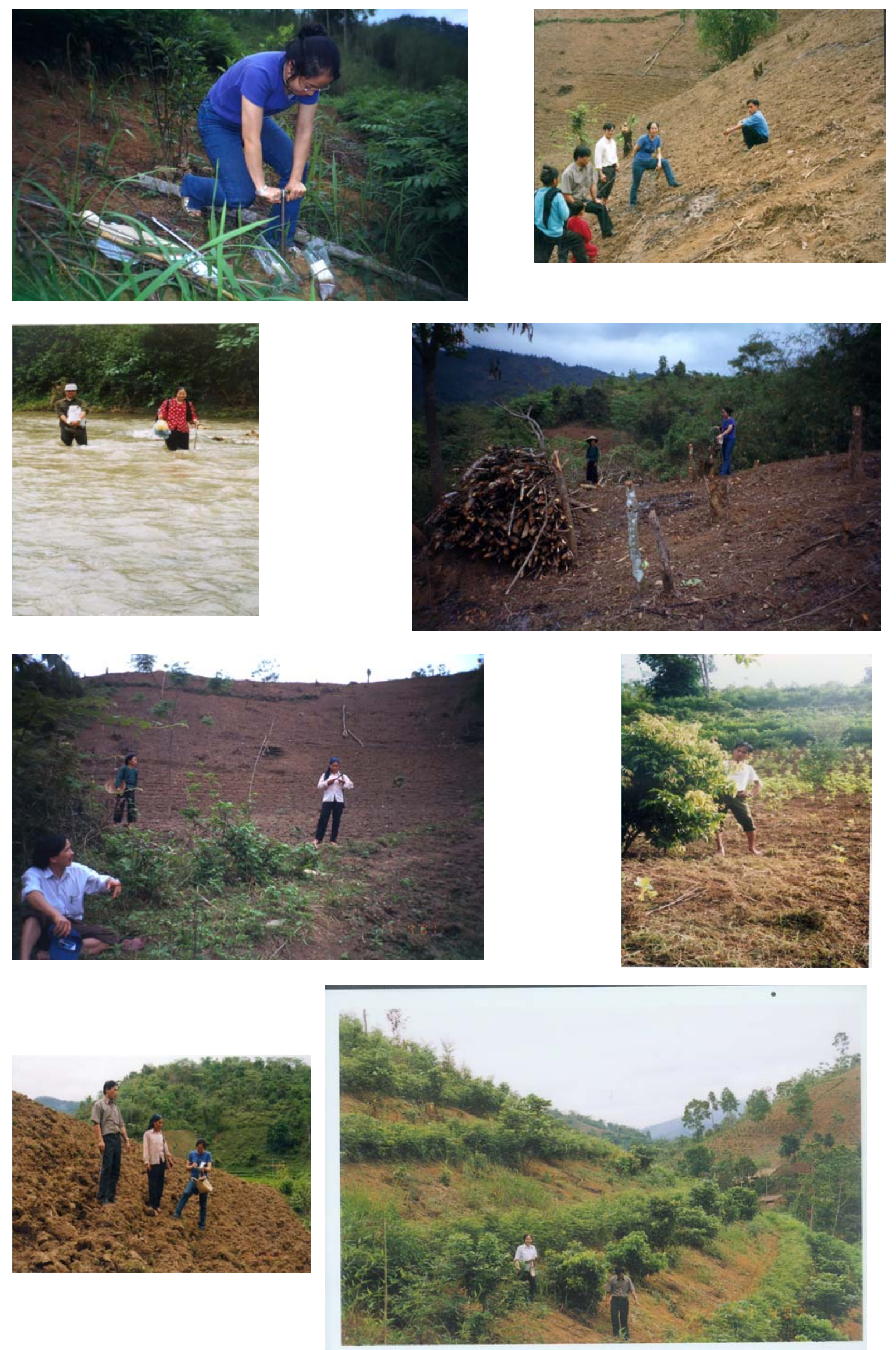


\section{LEBENSLAUF}

Name:

Do, Thi Lan

Geboren:

19. Januar 1972, in Son La Provinz

Eltern :

Do Xuan Mien und Do Thi Xuan

Staatsangehörigkeit: Vietnamesisch

Schule:

1977- 1982 Grundschule in Son La Provinz 1982- 1988 Gymnasium in Son La Provinz Juni 1988 Abitur

Studium:

10.1988- 4. 1993 - Thainguyen Universität, Vietnam

Abschluss Bacherlor. Ing. Agrarwissenschaft

10.1993- 9.1995- Hanoi Universität, Vietnam

Abschluss M.Sc „Master of sience“

Magisterarbeit: „Researching of limited sectors to paddy rice's productivity on valley land, Bacthai Province,, mit der Note sehr gut

Berufstätigkeit: $\quad$ 10.1995-10.1999 Dozentin in der Abteilung Bodenwissenschaft Thai nguyen Universität, Vietnam.

11.1999- 9.2000 Besuch eines Deutschkurses in Bochum als Stipendiatin des Ökumenischen Studienwerk.

Promotion : $\quad$ 10.2000- 03.2004 Stipendiatin von ÖSW. Betreuer : Prof. Dr. G. Gerold - Abteilung Landschaftsökologie am Geographischen Institut- Universität Göttingen.

Dissertation: $\quad$ Erhaltung Bodenfruchtbarkeit unter Anwendung angepasster Anbaussysteme in Bergregionen Vietnams.

Berufstätigkeit: $\quad$ ab April 2004 als Dozentin in der Abteilung Bodenwissenschaft, Fakultät Landmanagement - Thai nguyen Universität, Vietnam. 